\title{
Local minimization, variational evolution and $\Gamma$-convergence
}

\author{
Andrea Braides
}

Dipartimento di Matematica, Università di Roma 'Tor Vergata' via della ricerca scientifica 1, 00133 Roma (Italy) 


\section{Contents}

$\begin{array}{ll}\text { Preface } & 7\end{array}$

$\begin{array}{ll}\text { Introduction } & 9\end{array}$

1 Global minimization $\quad \mathbf{1 5}$

1.1 Upper and lower bounds . . . . . . . . . . . . . . . . . . 15

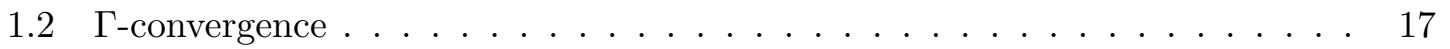

1.3 Convergence of minimum problems . . . . . . . . . . . . . . . 20

1.4 An example: homogenization . . . . . . . . . . . . . . . . 21

1.5 Higher-order $\Gamma$-limits and a choice criterion . . . . . . . . . . . 26

1.6 References to Chapter $1 \ldots \ldots \ldots$. . . . . . . . . . 30

2 Parameterized motion driven by global minimization 31

2.1 Damage models . . . . . . . . . . . . . . . . . . . . . 31

2.1.1 Damage of a homogeneous material . . . . . . . . . . . . 31

2.1.2 Homogenization of damage . . . . . . . . . . . . . 36

2.1.3 Homogenization of damage: dissipations leading to a commutability

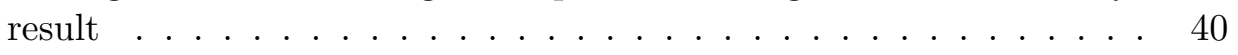

2.1.4 Conditions for commutability . . . . . . . . . . . . . . 43

2.1.5 Relaxed evolution . . . . . . . . . . . . . . . . . 44

2.2 Mielkian theory of rate-independent evolution . . . . . . . . . . . . 46

2.2 .1 Stability . . . . . . . . . . . . . . . . . . . 49

2.3 Francfort and Marigo's Variational Theory of Fracture . . . . . . . . . . 51

2.3.1 Homogenization of fracture . . . . . . . . . . . . . . . 53

2.4 References to Chapter $2 \ldots \ldots \ldots \ldots \ldots$

3 Local minimization as a selection criterion $\quad \mathbf{5 5}$

3.1 Equivalence by $\Gamma$-convergence . . . . . . . . . . . . . . 55

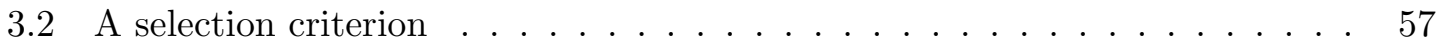

3.3 A 'quantitative' example: phase transitions . . . . . . . . . . . . . 58

3.4 A 'qualitative' example: Lennard-Jones atomistic systems . . . . . . . . . . 60 
3.5 A negative example: oscillating perimeters . . . . . . . . . . . . . . 65

3.6 References to Chapter $3 \ldots \ldots \ldots 6$. . . . . . . . . . . . . . . . .

4 Convergence of local minimizers $\quad 67$

4.1 Convergence to isolated local minimizers . . . . . . . . . . . . . 67

4.2 Two examples . . . . . . . . . . . . . . . . . . . . 68

4.3 Generalizations . . . . . . . . . . . . . . . . . . 71

4.4 References to Chapter $4 \ldots \ldots \ldots \ldots \ldots$

5 Small-scale stability $\quad \mathbf{7 9}$

5.1 Larsen's stable points . . . . . . . . . . . . . . . . . . 79

5.2 Stable sequences of functionals . . . . . . . . . . . . . . 81

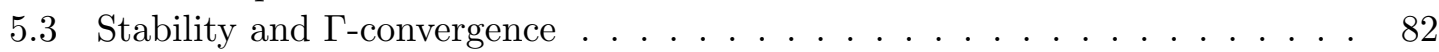

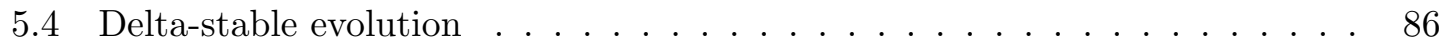

5.5 References to Chapter $5 \ldots \ldots \ldots \ldots$. . . . . . . . . . . . . 88

6 Minimizing movements $\quad 89$

6.1 An energy-driven implicit-time discretization . . . . . . . . . . . . . 89

6.2 Time-dependent minimizing movements . . . . . . . . . . . . . . 95

6.3 References to Chapter $6 \ldots \ldots \ldots \ldots$. . . . . . . . . . . 97

7 Minimizing movements along a sequence of functionals 99

7.1 Minimizing movements along a sequence . . . . . . . . . . . . . . 100

7.2 Commutability along 'fast-converging' sequences . . . . . . . . . . . . 101

7.2.1 Relaxed evolution . . . . . . . . . . . . . . . . . 105

7.3 An example: 'overdamped dynamics' of Lennard-Jones interactions . . . . . 106

7.4 Homogenization of minimizing movements . . . . . . . . . . . . . . . 110

7.4.1 Minimizing movements for piecewise-constant energies . . . . . . . . 110

7.4.2 A heterogeneous case . . . . . . . . . . . . . . . . 114

7.4.3 A proposal for some random models . . . . . . . . . . . . . . 117

7.5 Time-dependent minimizing movements . . . . . . . . . . . . . . 118

7.6 References to Chapter $7 \ldots \ldots \ldots \ldots$. . . . . . . . . . . . . . . . . . . . . . . . .

8 Geometric minimizing movements 121

8.1 Motion by mean curvature . . . . . . . . . . . . . . . . 121

8.2 A first (unsuccessful) generalization . . . . . . . . . . . . . . . . 122

8.3 A variational approach to curvature-driven motion . . . . . . . . . . . . 124

8.4 Homogenization of flat flows . . . . . . . . . . . . . . 125

8.4.1 Motion by crystalline curvature . . . . . . . . . . . . 125

8.5 Homogenization of oscillating perimeters . . . . . . . . . . . . 127

8.6 Flat flow with oscillating forcing term . . . . . . . . . . . . . . . 129

8.6.1 Flat flow with forcing term . . . . . . . . . . . . 129 
8.6.2 Homogenization of forcing terms . . . . . . . . . . . . 130

8.7 References to Chapter $8 \ldots \ldots \ldots \ldots \ldots$

9 Different time scales 135

9.1 Long-time behaviour . . . . . . . . . . . . . . . . . . . . . 135

9.2 Reversed time . . . . . . . . . . . . . . . . . . . . 144

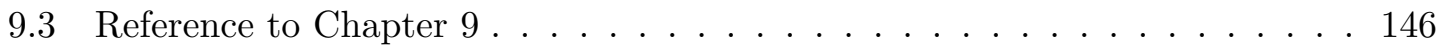

$\begin{array}{ll}10 \text { Stability theorems } & 147\end{array}$

10.1 Stability for convex energies . . . . . . . . . . . . . . . . . 147

10.1.1 Convergence estimates . . . . . . . . . . . . . . . . 147

10.1.2 Stability along sequences of convex energies . . . . . . . . . . 150

10.2 Sandier-Serfaty theory . . . . . . . . . . . . . . . . 154

10.2.1 Convergence of gradient flows . . . . . . . . . . . . . . 154

10.2.2 Convergence of stable critical points . . . . . . . . . . . . 157

10.3 References to Chapter $10 \ldots \ldots \ldots$. . . . . . . . . . . . 158

$\begin{array}{ll}\text { Index } & 161\end{array}$ 
CONTENTS 


\section{Preface}

These notes have been motivated by the interests of the author in variational problems depending on small parameters, for some of which a description based on a global minimization principle does not seem satisfactory. Such problems range from the derivation of physical theories from first principles to numerical problems involving energies with many local minima. Even though an asymptotic description of related global minimization problems can be given in terms of $\Gamma$-convergence, the $\Gamma$-limit often does not capture the behavior of local minimizers or of gradient flows. This failure is sometimes mentioned as the proof that $\Gamma$-convergence is 'wrong'. It may well be so. The author's standpoint is that it might nevertheless be a good starting point that may be systematically 'corrected'.

The author's program has been to examine the (few) results in the literature, and try to connect them with his own work in homogenization and discrete systems, where often the local minimization issues are crucial due to the oscillations of the energies. The directions of research have been

- find criteria that ensure the convergence of local minimizers and critical points. In case this does not occur then modify the $\Gamma$-limit into an equivalent $\Gamma$-expansion (as introduced by the author and L. Truskinovsky) in order to match this requirement. We note that in this way we 'correct' some limit theories, finding (or 'validating') other ones present in the literature;

- modify the concept of local minimizer, so that it may be more 'compatible' with the process of $\Gamma$-limit. One such concept is the $\delta$-stability of C. Larsen;

- treat evolution problems for energies with many local minima obtained by a timediscrete scheme (introducing the notion of 'minimizing movements along a sequence of functionals'). In this case the minimizing movement of the $\Gamma$-limit can be always obtained by a choice of the space and time-scale, but more interesting behaviors can be obtained at a critical ratio between them. In many cases a 'critical scale' can be computed and an effective motion, from which all other minimizing movements are obtained by scaling. Furthermore the choice of suitable $\Gamma$-converging sequences in the scheme above allows to address the issues of long-time behavior and backwards motion;

- examine the general variational evolution results that may be related to these mini-

mizing movements, in particular recent theories of quasistatic motion and gradent flow in metric spaces. 
The content of the present notes is taken from a series of lectures which formed a $\mathrm{PhD}$ course first given at Sapienza University of Rome from March to May 2012 and subsequently at the University of Pavia from November 2012 to January 2013. Those courses were addressed to an audience of students, some of which with an advanced background (meaning that they were already exposed to the main notions of the Calculus of Variations and of $\Gamma$-convergence), and researchers in the field of the Calculus of Variations and of Variational Evolution. This was an advanced course in that it was meant to address some current (or future) research issues rather than to discuss some subject systematically. Part of the notes has been also reworked during a ten-hour course at the University of Narvik on October 25-30, 2012.

Since the scope of the notes has been to highlight the phenomena and issues linked to local minimization and variational evolution, we have focused our attention on those aspects, rather than on the details of the $\Gamma$-convergence process, or the optimal hypotheses for the definition of gradient flows, for which we refer to the existing literature.

These notes would not have been written without the personal constant encouragement of Adriana Garroni, who is also responsible of the organization of the $\mathrm{PhD}$ course in Rome. I gratefully acknowledge the invitation of Enrico Vitali to give the $\mathrm{PhD}$ course in Pavia, his many interesting comments and his delightful hospitality. I greatly profited from the stimulating environments in both Departments; special thanks go to all the students who interacted during the course and the final exams. A precious direct contribution has been given by Adriana Garroni for many ideas about the homogenization of damage in Section 2.1, by Ulisse Stefanelli, who provided the material for most of Section 2.2 by giving a beautiful lecture on the subject during the course at Pavia, and by Luigi Ambrosio for the proofs in Section 10.1.1. I also acknowledge very fruitful discussions with Matteo Focardi, Chris Larsen, Alexander Mielke, Matteo Novaga, Andrey Piatnitski, Giuseppe Savaré and Lev Truskinovsky, which inspired many examples in these notes.

Rome, February 2013. 


\section{Introduction}

The theory of $\Gamma$-convergence was conceived by Ennio De Giorgi at the beginning of the 1970s. It originated from previous notions of convergence related mainly to elliptic operators as $G$-convergence or $H$-convergence or to convex functionals as Mosco convergence. The main issue in the definition of $\Gamma$-convergence is tracking the behaviour of global minimum problems (minimum values and minimizers) of a sequence $F_{\varepsilon}$ by the computation of an 'effective' minimum problem involving the (suitably defined) $\Gamma$-limit of this sequence. Even though the definition of such a limit is local (in that in defining its value at a point $x$ we only take into account sequences converging to $x$ ), its computation in general does not describe the behaviour of local minimizers of $F_{\varepsilon}$ (i.e., points $x_{\varepsilon}$ which are absolute minimizers of the restriction of $F_{\varepsilon}$ to a small neighbourhood of the point $x_{\varepsilon}$ itself). The possible situation, in a very simplified picture, is that in Figure 1, where the original $F_{\varepsilon}$ possess
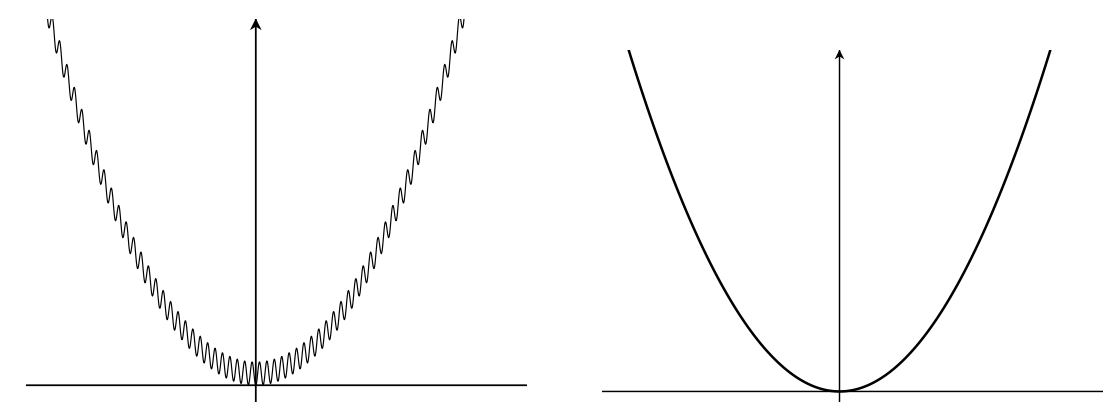

Figure 1: many local minima may disappear in the limit

many local minimizers, some (or all) of which are 'integrated out' in the $\Gamma$-convergence process (note that this happens even when the oscillations depth does not vanish). A notable exception is when we have an isolated local minimizer $x$ of the $\Gamma$-limit: in that case we may track the behaviour of local minimizers as absolute minimizers of $F_{\varepsilon}$ restricted to a fixed neighbourhood of $x$ and conclude the existence of local minimizers for $F_{\varepsilon}$ close to $x$. The possibility of the actual application of such a general principle has been envisaged by Kohn and Sternberg, who first used it to deduce the existence of local minimizers of the Allen-Cahn equation by exhibiting local area minimizing sets. 
A recent different but related direction of research concerns the study of gradient flows. A general variational theory based on the solution of Euler schemes has been developed by Ambrosio, Gigli and Savaré. The stability of such schemes by $\Gamma$-perturbations is possible in the absence of local minimizers which could generate 'pinned' flows (i.e., stationary solutions or solutions 'attracted' by a local minimum) that are not detected by the limit. Conditions that guarantee such a stability are of convexity type on the energies. These conditions can be removed under other special assumptions on the gradient flows and for 'well-prepared' initial data following the scheme proposed for Ginzburg-Landau energies by Sandier and Serfaty. Unfortunately, as remarked by those authors, the applicability of this scheme is often hard to verify.

Taking the above-mentioned results as a starting point we have explored some different directions. The standpoint of the analysis is that even though the $\Gamma$-limit may not give the correct description of the effect of local minimizers, it may nevertheless be 'corrected' in some systematic way.

After introducing the main notions of $\Gamma$-convergence in Chapter 1 and the general results on convergence of global minimum problems, in Chapter 2 we have examined another question related to global minimization, concerning the behavior of quasistatic motions, where the functions $u_{\varepsilon}=u_{\varepsilon}(t, x)$ minimize at each value of the parameter $t$ (which is understood as a 'slow time') a total energy of the form $F_{\varepsilon}(u, v)+D_{\varepsilon}(v)$ subject to a $t$-depending forcing condition. Here, $v$ is an additional parameter satisfying some monotonicity constraint and $D_{\varepsilon}$ is a dissipation. We examine conditions that imply that the separate $\Gamma$-convergences of $F_{\varepsilon}$ to $F$ and of $D_{\varepsilon}$ to $D$ guarantee the convergence of $u_{\varepsilon}$ to a quasi static motion related to $F$ and $D$, as envisaged by Mielke, Roubiček and Stefanelli. In general such conditions do not hold, and a relaxed formulation taking into account the interaction of $F_{\varepsilon}$ and $D_{\varepsilon}$ must be used. In this case, quasistatic motion turns out to be compatible with $\Gamma$-convergence provided that the latter is understood as that of the functionals $G_{\varepsilon}(u, v)=F_{\varepsilon}(u, v)+D_{\varepsilon}(v)$ of the variable $(u, v)$.

In Chapter 3 we have dealt with another case when the global minimization arguments can be 'localized', which is the case of isolated local minimizers of the $\Gamma$-limit, as recalled above. We have noted that this principle can also be applied to scaled energies (e.g., to higher-order $\Gamma$-limits, or to 'blown-up' energies) obtaining existence of multiple local minimizers for the $\Gamma$-converging functionals.

A first issue beyond global minimization is introduced in Chapte 4 and takes into account the notion of 'equivalence by $\Gamma$-convergence' as introduced and studied by Braides and Truskinovsky: in the case that a $\Gamma$-limit or a $\Gamma$-development may be insufficient to capture some desired feature of the minimum problems $F_{\varepsilon}$ we may introduce equivalent energies $\widetilde{F}_{\varepsilon}$. These energies still integrate out the unimportant details of $F_{\varepsilon}$ but maintain the desired feature and are equivalent to the original $F_{\varepsilon}$ in that they have the same $\Gamma$ limit or $\Gamma$-development. One of the conditions that may be required to $\widetilde{F}_{\varepsilon}$ is that they have the same landscape of local minimizers as $F_{\varepsilon}$. As an example, we highlight that a $\Gamma$ - 
development taking into account interactions between neighbouring transitions recovers the local minimizers of Allen-Cahn energies that are integrated out by the usual sharp-interface models of phase transitions.

Another issue is the problem of distinguishing 'meaningful local minimizers' from those that may 'rightfully' considered to disappear in the limit. Taking Figure 1 as a pictorial example, local minimizers deriving from vanishing oscillations should be considered as different from those 'trapped' by energy barriers. To that end in Chapter 5 we study the notion of $\delta$-stable state as recently introduced by C. Larsen, and the related notion of stable sequences of energies. We show that $\Gamma$-convergence allows to exhibit classes of stable sequences.

Linked to the study of local minimizers is the variational motion defined by the limit of Euler schemes at vanishing time step. This motion has been usually defined for a single functional $F$ (and is sometimes referred to as a minimizing movement), by introducing a time step $\tau$ and define discrete trajectories $\left(u_{k}^{\tau}\right)$ iteratively as solutions of

$$
\min \left\{F(u)+\frac{1}{2 \tau}\left\|u-u_{k-1}^{\tau}\right\|^{2}\right\}
$$

(for simplicity assume that the energies be defined on a Hilbert space, and $\|u\|$ the related norm). A minimizing movement is a suitably defined continuum limit of such discrete trajectories. These concept are introduced in Chapter 6 together with their analogues for time-dependent energies.

In Chapter 7 we examine a variation of the minimizing-movement scheme with two parameters: one is the time step $\tau$, and the second one is the parameter $\varepsilon$ (that we may ofter regard as a space scale) appearing in the $\Gamma$-converging sequence $F_{\varepsilon}$. The Euler scheme is then applied at fixed $\tau$ with $\varepsilon=\varepsilon(\tau)$ and $F_{\varepsilon}$ in place of $F$, so that the resulting discrete trajectories $\left(u_{k}^{\varepsilon}\right)$ defined iteratively as solutions of

$$
\min \left\{F_{\varepsilon}(u)+\frac{1}{2 \tau}\left\|u-u_{k-1}^{\varepsilon}\right\|^{2}\right\}
$$

may depend on the interaction between the two scales. A general result, directly derived from the properties of $\Gamma$-convergence allows to deduce the existence of a 'fast' space scale such that the limit of the $\varepsilon-\tau$ Euler scheme is just a minimizing movement for the $\Gamma$-limit. For 'slow' space scales the motion is often 'pinned' at local minimizers. This observation highlights the existence of one or more critical $\varepsilon-\tau$ regimes which capture the most interesting features of the motion connected to these energies. We show examples where an 'effective' motion is obtained such that all minimizing movements are obtained by scaling.

An important class of minimizing movements are geometric minimizing movements treated in Chapter 8. The prototype of such a geometric motion is the motion by mean curvature obtained as a minimizing movement starting from the perimeter functional by Almgren, Taylor and Wang. In this case the scheme must be generalized to cover the case 
of an 'asymmetric' distance. This generalized scheme can be applied to treat sequences of perimeter energies.

Another possible phenomenon for these Euler schemes for $\Gamma$-converging energies is the existence of more superposed time scales, whose motions can themselves be interpreted as derived from Euler schemes for scaled functionals $F_{\varepsilon} / \lambda_{\varepsilon}$. Moreover, an appropriate choice of $\Gamma$-approximating sequences to a given $F$ may be used to define a 'backward' motion. These issues are examined in Chapter 9.

Finally, in Chapter 10 we examine stability issues. The first one is linked to the theory of gradient flows elaborated by Ambrosio, Gigli and Savaré. Using their approach we can show that for convex equicoercive energies $\Gamma$-convergence always commutes with the minimizing-movement scheme. This is also true for some non-convex energies following the Sandier-Serfaty approach.

The course is organized around some fundamental examples. We have analyzed a number of prototypical $\Gamma$-converging sequences $F_{\varepsilon}: X \rightarrow[-\infty,+\infty]$, highlighting different phenomena.

1. Elliptic homogenization:

$$
F_{\varepsilon}(u)=\int_{\Omega} a\left(\frac{x}{\varepsilon}\right)|\nabla u|^{2} d x
$$

with $a$ 1-periodic. In this case $X$ is (a subset of) $H^{1}(\Omega)$. The inhomogeneity a represents the fine properties of a composite medium. These energies are convex so that they do not possess local (non-global) minimizers. We may nevertheless introduce non-trivial perturbations for which we exhibit an isolated local minimizer for the $\Gamma$-limit, deducing existence of nontrivial local minimizers. Their convexity ensures that for the functionals $F_{\varepsilon} \Gamma$-convergence commutes with the minimizing movement schemes. As an application we can deduce a parabolic homogenization theorem.

2. Oscillating metrics:

$$
F_{\varepsilon}(u)=\int_{0}^{1} a\left(\frac{u}{\varepsilon}\right)\left|u^{\prime}\right| d t
$$

with $a$ as above and $X$ a subspace of $W^{1, \infty}\left([0,1] ; \mathbb{R}^{n}\right)$, and the analog oscillating perimeter energies

$$
F_{\varepsilon}(A)=\int_{\partial A} a\left(\frac{x}{\varepsilon}\right) d \mathcal{H}^{d-1}(x) .
$$

Here we are interested in the overall metric properties, or, in the case of perimeters, of the averaged interfacial energies in a composite medium. These energies have many local minimizers (which tend to be dense as $\varepsilon \rightarrow 0$ ), which are not stable as $\varepsilon \rightarrow 0$. As a consequence we have a variety of limit minimizing movements that range from trivial (i.e., constant) motions, to 'crystalline' motion, passing through interesting regimes where the scale $\varepsilon$ interacts with the timescale $\tau$. In the case of perimeter energies the limit motion 
is a kind of motion by curvature with a discontinuous dependence of the velocity on the curvature. Conversely, they may be 'incompatible' with simple types of dissipation, and lead to relaxed quasi static evolutions.

3. Van-der-Waals theory of phase transitions:

$$
F_{\varepsilon}(u)=\int_{\Omega}\left(\frac{W(u)}{\varepsilon}+\varepsilon|\nabla u|^{2}\right) d x
$$

with $X=H^{1}(\Omega)$. Here $W$ is a double-well potential with minima in \pm 1 . In one dimension this is an example where the limit sharp-interface energy has many local minimizers (actually, all functions in the domain of the limit are local minimizers), so that a correction of the $\Gamma$-limit may be required to take into account the interaction of interfaces, through an exponentially decaying term. It must be noted that this corrected energy also provides an example of an exponentially scaled time-scale at which we have motion of interfaces (which otherwise stay pinned).

4. Atomistic theories: for a $1 D$ chain of atoms

$$
F_{\varepsilon}(u)=\sum_{i} J\left(u_{i}-u_{i-1}\right)
$$

where $J$ is an interatomic potential (e.g., the Lennard-Jones potential), and $u_{i}$ represent the position of the $i$-th atom of a chain of $N$ atoms, ordered with $u_{i}>u_{i-1}$. Here $\varepsilon=1 / N$. Starting from Lennard-Jones atomic interactions we show that the resulting $\Gamma$-limit in one dimension (the Mumford-Shah functional or Griffith brittle fracture energy) must be modified to a Barenblatt cohesive fracture energy to maintain the features of the local minimizers. Even though these energies are not convex, the Euler scheme commutes with $\Gamma$ convergence. Another feature of the variational motion is the appearance of a rescaled time scale, in which 'multiple fractures' (that correspond to 'extra' local minimizers introduced by the limit process) tend to interact. A similar behavior is shown for Perona-Malik scaled energies, when $J(z)$ is of the form $\log \left(1+z^{2}\right)$.

We believe that the examples and results highlighted provide a rather complex overview of the type of issues these notes want to be a stimulus to further analyze. 


\section{Chapter 1}

\section{Global minimization}

The issues related to the behavior of global minimization problems along a sequence of functionals $F_{\varepsilon}$ are by now well understood, and mainly rely on the concept of $\Gamma$-limit. In this chapter we review this notion, which will be the starting point of our analysis. We will mainly be interested in the properties of $\Gamma$-limits with respect to the convergence of minimization problems; further properties of $\Gamma$-limits will be recalled when necessary.

\section{$1.1 \quad$ Upper and lower bounds}

Here and afterwards $F_{\varepsilon}$ will be functionals defined on a separable metric (or metrizable) space $X$, if not further specified.

Definition 1.1.1 (lower bound) We say that $F$ is a lower bound for the family $\left(F_{\varepsilon}\right)$ if for all $u \in X$ we have

$$
F(u) \leq \liminf _{\varepsilon \rightarrow 0} F_{\varepsilon}\left(u_{\varepsilon}\right) \text { for all } u_{\varepsilon} \rightarrow u,
$$

or, equivalently, $\underset{\varepsilon \rightarrow 0}{F(u)} \leq F_{\varepsilon}\left(u_{\varepsilon}\right)+o(1)$ for all $u_{\varepsilon} \rightarrow u$.

The inequality (LB) is usually referred to as the liminf inequality.

If $F$ is a lower bound we obtain a lower bound also for minimum problems on compact sets.

Proposition 1.1.2 Let $F$ be a lower bound for $F_{\varepsilon}$ and $K$ be a compact subset of $X$. Then

$$
\inf _{K} F \leq \liminf _{\varepsilon \rightarrow 0} \inf _{K} F_{\varepsilon}
$$

Proof. Let $u_{\varepsilon_{k}} \in K$ be such that $u_{\varepsilon_{k}} \rightarrow \bar{u}$ and

$$
\lim _{k} F_{\varepsilon_{k}}\left(u_{\varepsilon_{k}}\right)=\liminf _{\varepsilon \rightarrow 0} \inf _{K} F_{\varepsilon} .
$$


We set

$$
\widetilde{u}_{\varepsilon}= \begin{cases}u_{\varepsilon_{k}} & \text { if } \varepsilon=\varepsilon_{k} \\ \bar{u} & \text { otherwise. }\end{cases}
$$

Then by (LB) we have

$$
\inf _{K} F \leq F(\bar{u}) \leq \liminf _{\varepsilon \rightarrow 0} F_{\varepsilon}\left(\widetilde{u}_{\varepsilon}\right) \leq \lim _{k} F_{\varepsilon_{k}}\left(u_{\varepsilon_{k}}\right)=\liminf _{\varepsilon \rightarrow 0} \inf _{K} F_{\varepsilon}
$$

as desired.

Remark 1.1.3 Note that the hypothesis that $K$ be compact cannot altogether be removed. A trivial example on the real line is:

$$
F_{\varepsilon}(x)= \begin{cases}-1 & \text { if } x=1 / \varepsilon \\ 0 & \text { otherwise }\end{cases}
$$

Then $F=0$ is a lower bound according to Definition 1.1.1, but (1.1) fails if we take $\mathbb{R}$ in place of $K$.

Remark 1.1.4 The hypothesis that $K$ be compact can be substituted by the hypothesis that $K$ be closed and the sequence $\left(F_{\varepsilon}\right)$ be equi-coercive; i.e., that

$$
\text { if } \sup _{\varepsilon} F_{\varepsilon}\left(u_{\varepsilon}\right)<+\infty \text { then }\left(u_{\varepsilon}\right) \text { is precompact, }
$$

the proof being the same.

Definition 1.1.5 (upper bound) We say that $F$ is a upper bound for the family $\left(F_{\varepsilon}\right)$ if for all $u \in X$ we have

there exists $u_{\varepsilon} \rightarrow u$ such that $F(u) \geq \limsup _{\varepsilon \rightarrow 0} F_{\varepsilon}\left(u_{\varepsilon}\right)$.

or, equivalently, $F(u) \geq F_{\varepsilon}\left(u_{\varepsilon}\right)+o(1)$.

The inequality (UB) is usually referred to as the limsup inequality.

If $F$ is an upper bound for $F_{\varepsilon}$ we obtain an upper bound also for the corresponding minimum problems on open sets.

Proposition 1.1.6 Let $F$ be an upper bound for $F_{\varepsilon}$ and $A$ be an open subset of $X$. Then

$$
\inf _{A} F \geq \limsup _{\varepsilon \rightarrow 0} \inf _{A} F_{\varepsilon}
$$


Proof. The proof is immediately derived from the definition after remarking that if $u \in A$ then we may suppose also that $u_{\varepsilon} \in A$ so that

$$
F(u) \geq \limsup _{\varepsilon \rightarrow 0} F_{\varepsilon}\left(u_{\varepsilon}\right) \geq \limsup _{\varepsilon \rightarrow 0} \inf _{A} F_{\varepsilon}
$$

and (1.4) follows by the arbitrariness of $u$.

Remark 1.1.7 Again, note that the hypothesis that $A$ be open cannot be removed. A trivial example on the real line is:

$$
F_{\varepsilon}(x)= \begin{cases}1 & \text { if } x=0 \\ 0 & \text { otherwise }\end{cases}
$$

(independent of $\varepsilon$ ). Then $F=0$ is an upper bound according to Definition 1.1.5 (and also a lower bound!), but (1.4) fails taking $A=\{0\}$.

Note that in the remark above 0 is an upper bound for $F_{\varepsilon}$ at 0 even though $F_{\varepsilon}(0)=1$ for all $\varepsilon$, which trivially shows that an upper bound at a point can be actually (much) lower that any element of the family $F_{\varepsilon}$ at that point.

\section{$1.2 \quad \Gamma$-convergence}

In this section we introduce the concept of $\Gamma$-limit.

Definition 1.2.1 ( $\Gamma$-limit) We say that $F$ is the $\Gamma$-limit of the sequence $\left(F_{\varepsilon}\right)$ if it is both a lower and an upper bound according to Definitions 1.1.1 and 1.1.5.

If (LB) and (UB) hold at a point $u$ then we say that $F$ is the $\Gamma$-limit at $u$, and we write

$$
F(u)=\Gamma-\lim _{\varepsilon \rightarrow 0} F_{\varepsilon}(u) .
$$

Note that this notation does does not imply that $u$ is in any of the domains of $F_{\varepsilon}$, even if $F(u)$ is finite.

Remark 1.2.2 (alternate upper bound inequalities) If $F$ is a lower bound then requiring that (UB) holds is equivalent to any of the following

$$
\text { there exists } u_{\varepsilon} \rightarrow u \text { such that } F(u)=\lim _{\varepsilon \rightarrow 0} F_{\varepsilon}\left(u_{\varepsilon}\right) \text {; }
$$

for all $\eta>0$ there exists $u_{\varepsilon} \rightarrow u$ such that $F(u)+\eta \geq \limsup _{\varepsilon \rightarrow 0} F_{\varepsilon}\left(u_{\varepsilon}\right)$.

The latter is called the approximate limsup inequality, and is more handy in computations. A sequence satisfying (RS) is called a recovery sequence. The construction of a recovery sequence is linked to an ansatz on its form. The description of this ansatz gives an insight of the relevant features of the energies (oscillations, concentration, etc.) and is usually given on a subclass of $u$ for which it is easier to prove its validity, while for general $u$ one proceeds by a density argument. 
Example 1.2.3 We analyze some simple examples on the real line.

1. From Remark 1.1.7 we see that the constant sequence

$$
F_{\varepsilon}(x)= \begin{cases}1 & \text { if } x=0 \\ 0 & \text { otherwise }\end{cases}
$$

$\Gamma$-converges to the constant 0 ; in particular this is a constant sequence not converging to itself.

2. The sequence

$$
F_{\varepsilon}(x)= \begin{cases}1 & \text { if } x=\varepsilon \\ 0 & \text { otherwise }\end{cases}
$$

again $\Gamma$-converges to the constant 0 . This is clearly a lower and an upper bound at all $x \neq 0$. At $x=0$ any sequence $x_{\varepsilon} \neq \varepsilon$ is a recovery sequence.

3. The sequence

$$
F_{\varepsilon}(x)= \begin{cases}-1 & \text { if } x=\varepsilon \\ 0 & \text { otherwise }\end{cases}
$$

$\Gamma$-converges to

$$
F(x)= \begin{cases}-1 & \text { if } x=0 \\ 0 & \text { otherwise. }\end{cases}
$$

Again, $F$ is clearly a lower and an upper bound at all $x \neq 0$. At $x=0$ the sequence $x_{\varepsilon}=\varepsilon$ is a recovery sequence.

4. Take the sum of the energies in Examples 2 and 3 above. This is identically 0 , so is its limit, while the sum of the $\Gamma$-limits is the function $F$ in Example 3. The same function $F$ is obtained as the $\Gamma$-limit by taking the function $G_{\varepsilon}(x)=F_{\varepsilon}(x)+F_{\varepsilon}(-x)\left(F_{\varepsilon}\right.$ in Example $3)$.

5. Let $F_{\varepsilon}(x)=\sin (x / \varepsilon)$. Then the $\Gamma$-limit is the constant -1 . This is clearly a lower bound. A recovery sequence for a fixed $x$ is $x_{\varepsilon}=2 \pi \varepsilon\lfloor x /(2 \pi \varepsilon)\rfloor-\varepsilon \pi / 2(\lfloor t\rfloor$ is the integer part of $t$ ).

The following fundamental property of $\Gamma$-convergence derives directly from its definition

Proposition 1.2.4 (stability under continuous perturbations) Let $F_{\varepsilon} \Gamma$-converge to $F$ and $G_{\varepsilon}$ converge continuously to $G$ (i.e., $G_{\varepsilon}\left(u_{\varepsilon}\right) \rightarrow G(u)$ if $u_{\varepsilon} \rightarrow u$ ); then $F_{\varepsilon}+G_{\varepsilon} \rightarrow$ $F+G$.

Note that this proposition applies to $G_{\varepsilon}=G$ if $G$ is continuous, but is in general false for $G_{\varepsilon}=G$ even if $G$ is lower semicontinuous.

Example 1.2.5 The functions $\sin (x / \varepsilon)+x^{2}+1 \Gamma$-converge to $x^{2}$. In this case we may apply the proposition above with $F_{\varepsilon}(x)=\sin (x / \varepsilon)$ (see Example 1.2.3(5)) and $G_{\varepsilon}(x)=x^{2}+1$. Note for future reference that $F_{\varepsilon}$ has countably many local minimizers, which tend to be dense in the real line, while $F$ has only one global minimizer. 
It may be useful to define the lower and upper $\Gamma$-limits, so that the existence of a $\Gamma$-limit can be viewed as their equality

Definition 1.2.6 (lower and upper $\Gamma$-limits) We define

$$
\begin{aligned}
& \Gamma-\liminf _{\varepsilon \rightarrow 0} F_{\varepsilon}(u)=\inf \left\{\liminf _{\varepsilon \rightarrow 0} F_{\varepsilon}\left(u_{\varepsilon}\right): u_{\varepsilon} \rightarrow u\right\} \\
& \Gamma-\limsup F_{\varepsilon}(u)=\inf \left\{\limsup _{\varepsilon \rightarrow 0} F_{\varepsilon}\left(u_{\varepsilon}\right): u_{\varepsilon} \rightarrow u\right\}
\end{aligned}
$$

Remark 1.2.7 1 . We immediately obtain that the $\Gamma$-limit exists at a point $u$ if and only if

$$
\Gamma-\liminf _{\varepsilon \rightarrow 0} F_{\varepsilon}(u)=\Gamma-\limsup _{\varepsilon \rightarrow 0} F_{\varepsilon}(u) .
$$

2. Comparing with the trivial sequence $u_{\varepsilon}=u$ we obtain

$$
\Gamma-\liminf _{\varepsilon \rightarrow 0} F_{\varepsilon}(u) \leq \liminf _{\varepsilon \rightarrow 0} F_{\varepsilon}(u)
$$

(and analogously for the $\Gamma$-limsup). More in general, note that the $\Gamma$-limit depends on the topology on $X$. If we change topology, converging sequences change and the value of the $\Gamma$-limit changes. A weaker topology will have more converging sequences and the value will decrease, a stronger topology will have less converging sequences and the value will increase. The pointwise limit above corresponding to the $\Gamma$-limit with respect to the discrete topology.

3. From the formulas above it is immediate to check that a constant sequence $F_{\varepsilon}=F$ $\Gamma$-converges to itself if and only if $F$ is lower semicontinuous; i.e., (LB) holds with $F_{\varepsilon}=F$. Indeed (LB) equivalent to the validity of (1.5), while $F$ is always an upper bound. More in general a constant sequence $F_{\varepsilon}=F$ converges to the lower-semicontinuous envelope $\bar{F}$ of $F$ defined by

$$
\bar{F}(u)=\max \{G: G \leq F, G \text { is lower semicontinuous }\} ;
$$

in particular the $\Gamma$-limit is a lower-semicontinuous function.

4. It may be convenient to notice that the upper and lower limits are lower-semicontinuous functions and, with the notation just introduced, that

$$
\begin{aligned}
\Gamma-\liminf _{\varepsilon \rightarrow 0} F_{\varepsilon}(u) & =\Gamma-\liminf _{\varepsilon \rightarrow 0} \overline{F_{\varepsilon}}(u) \\
\Gamma-\limsup _{\varepsilon \rightarrow 0} F_{\varepsilon}(u) & =\Gamma-\limsup _{\varepsilon \rightarrow 0} \overline{F_{\varepsilon}}(u) ;
\end{aligned}
$$

that is, $\Gamma$-limits are unchanged upon substitution of $F_{\varepsilon}$ with its lower-semicontinuous envelope. These properties are an important observation for the actual computation of the 
$\Gamma$-limit, since in many cases lower-semicontinuous envelopes satisfy structural properties that make them easier to handle. As an example we may consider (homogeneous) integral functionals of the form

$$
F(u)=\int_{\Omega} f(u) d x
$$

defined on $L^{1}(\Omega)$ equipped with the weak topology. Under some growth conditions the $\Gamma$-limits can be computed with respect to the weak topology on bounded sets of $L^{1}(\Omega)$, which is metrizable. In this case, the lower-semicontinuous envelope of $F$ is

$$
F(u)=\int_{\Omega} f^{* *}(u) d x
$$

where $f^{* *}$ is the convex and lower-semicontinuous envelope of $f$; i.e.,

$$
f^{* *}=\max \{g: g \leq f, g \text { is lower-semicontinuous and convex }\} .
$$

In particular convexity is a necessary condition for $\Gamma$-limits of the integral form above.

\subsection{Convergence of minimum problems}

As we have already remarked, the $\Gamma$-convergence of $F_{\varepsilon}$ will not imply convergence of minimizers if minimizers (or 'almost minimizers') do not converge. It is necessary then to assume a compactness (or 'mild coerciveness') property as follows:

$$
\text { there exists a precompact sequence }\left(u_{\varepsilon}\right) \text { with } F_{\varepsilon}\left(u_{\varepsilon}\right)=\inf F_{\varepsilon}+o(1) \text {, }
$$

which is implied by the following stronger condition

$$
\text { there exists a compact set } K \text { such that } \inf F_{\varepsilon}=\inf _{K} F_{\varepsilon} \text { for all } \varepsilon>0 \text {. }
$$

This condition is implied by the equi-coerciveness hypothesis (1.3); i.e., if for all $c$ there exists a compact set $K$ such that the sublevel sets $\left\{F_{\varepsilon} \leq c\right\}$ are all contained in $K$. To check that (1.10) is stronger than (1.9) consider $F_{\varepsilon}(x)=\varepsilon e^{x}$ on the real line: any converging sequence satisfies (1.9) but (1.10) does not hold.

By arguing as for Propositions 1.1.2 and 1.1.6 we will deduce the convergence of minima. This result is further made precise in the following theorem.

Theorem 1.3.1 (Fundamental Theorem of $\Gamma$-convergence) Let $\left(F_{\varepsilon}\right)$ satisfy the compactness property (1.9) and $\Gamma$-converge to $F$. Then

(i) $F$ admits minimum, and $\min F=\lim _{\varepsilon \rightarrow 0} \inf F_{\varepsilon}$

(ii) if $\left(u_{\varepsilon_{k}}\right)$ is a minimizing sequence for some subsequence $\left(F_{\varepsilon_{k}}\right)$ (i.e., is such that $\left.F_{\varepsilon_{k}}\left(u_{\varepsilon_{k}}\right)=\inf F_{\varepsilon}+o(1)\right)$ which converges to some $\bar{u}$ then its limit point is a minimizer for $F$. 
Proof. By condition (1.9) we can argue as in the proof of Proposition 1.1.2 with $K=X$ and also apply Proposition 1.1.6 with $A=X$ to deduce that

$$
\inf F \geq \limsup _{\varepsilon \rightarrow 0} \inf F_{\varepsilon} \geq \liminf _{\varepsilon \rightarrow 0} \inf F_{\varepsilon} \geq \inf F .
$$

We then have that there exists the limit

$$
\lim _{\varepsilon \rightarrow 0} \inf F_{\varepsilon}=\inf F .
$$

Since from (1.9) there exists a minimizing sequence $\left(u_{\varepsilon}\right)$ from which we can extract a converging subsequence, it suffices to prove (ii). We can then follow the proof of Proposition 1.1.2 to deduce as in (1.2) that

$$
\inf F \leq F(\bar{u}) \leq \lim _{k} F_{\varepsilon_{k}}\left(u_{\varepsilon_{k}}\right)=\lim _{\varepsilon \rightarrow 0} \inf F_{\varepsilon}=\inf F
$$

i.e., $F(\bar{u})=\inf F$ as desired.

Corollary 1.3.2 In the hypotheses of Theorem 1.3.1 the minimizers of $F$ are all the limits of converging minimizing sequences.

Proof. If $\bar{u}$ is a limit of a converging minimizing sequence then it is a minimizer of $F$ by (ii) in Theorem 1.3.1. Conversely, if $\bar{u}$ is a minimizer of $F$, then every its recovery sequence $\left(u_{\varepsilon}\right)$ is a minimizing sequence.

Remark 1.3.3 Trivially, it is not true that all minimizers of $F$ are limits of minimizers of $F_{\varepsilon}$, since this is not true even for (locally) uniformly converging sequences on the line. Take for example:

1) $F_{\varepsilon}(x)=\varepsilon x^{2}$ or $F_{\varepsilon}(x)=\varepsilon e^{x}$ and $F(x)=0$. All points minimize the limit but only $x=0$ minimizes $F_{\varepsilon}$ in the first case, and we have no minimizer for the second case;

2) $F(x)=\left(x^{2}-1\right)^{2}$ and $F_{\varepsilon}(x)=F(x)+\varepsilon(x-1)^{2} . F$ is minimized by 1 and -1 , but the only minimum of $F_{\varepsilon}$ is 1 . Note however that -1 is the limit of strong local minimizers for $F_{\varepsilon}$.

\subsection{An example: homogenization}

The theory of homogenization of integral functional is a very wide subject in itself. We will refer to monographs on the subject for details if needed. In this context, we want only to highlight some facts that will be needed in the sequel and give a hint of the behaviour in the case of elliptic energies. 
We consider $a: \mathbb{R}^{n} \rightarrow[\alpha, \beta]$, with $0<\alpha<\beta<+\infty$ 1-periodic in the coordinate directions, and the integrals

$$
F_{\varepsilon}(u)=\int_{\Omega} a\left(\frac{x}{\varepsilon}\right)|\nabla u|^{2} d x
$$

defined in $H^{1}(\Omega)$, where $\Omega$ is a bounded open subset of $\mathbb{R}^{n}$. The computation of the $\Gamma$ limit of $F_{\varepsilon}$ is referred to as their homogenization, implying that a simpler 'homogeneous' functional can be used to capture the relevant features of $F_{\varepsilon}$. The limit can be computed both with respect to the $L^{1}$ - topology, but it can also be improved; e.g., in 1D it coincides with the limit in the $L^{\infty}$ topology. This means that the liminf inequality holds for $u_{\varepsilon}$ converging in the $L^{1}$ topology, while there exists a recovery sequence with $u_{\varepsilon}$ tending to $u$ in the $L^{\infty}$ sense.

An upper bound is given by the pointwise limit of $F_{\varepsilon}$, whose computation in this case can be obtained by the following non-trivial but well-known result.

Proposition 1.4.1 (Riemann-Lebesgue lemma) The functions $a_{\varepsilon}(x)=a\left(\frac{x}{\varepsilon}\right)$ converge weakly* in $L^{\infty}$ to their average

$$
\bar{a}=\int_{(0,1)^{n}} a(y) d y
$$

For fixed $u$ the pointwise limit of $F_{\varepsilon}(u)$ is then simply $\bar{a} \int_{\Omega}|\nabla u|^{2} d x$, which then gives an upper bound for the $\Gamma$-limit.

In a one-dimensional setting, the $\Gamma$-limit is completely described by $a$, and is given by

$$
F_{\text {hom }}(u)=\underline{a} \int_{\Omega}\left|u^{\prime}\right|^{2} d x, \quad \text { where } \quad \underline{a}=\left(\int_{0}^{1} \frac{1}{a(y)} d y\right)^{-1}
$$

is the harmonic mean of $a$. We briefly sketch a proof which gives the ansatz for recovery sequences. We check the limit inequality by fixing $u_{\varepsilon} \rightarrow u$. Suppose for the sake of simplicity that $N=1 / \varepsilon \in \mathbb{N}$, and write

$$
\begin{aligned}
F_{\varepsilon}\left(u_{\varepsilon}\right) & =\sum_{i=1}^{N} \int_{\varepsilon(i-1)}^{\varepsilon i} a\left(\frac{x}{\varepsilon}\right)\left|u_{\varepsilon}^{\prime}\right|^{2} d x \\
& \geq \sum_{i=1}^{N} \varepsilon \min \left\{\int_{0}^{1} a(y)\left|v^{\prime}\right|^{2} d y: v(1)-v(0)=\frac{u_{\varepsilon}(\varepsilon i)-u_{\varepsilon}(\varepsilon(i-1))}{\varepsilon}\right\} \\
& =\underline{a} \sum_{i=1}^{N} \varepsilon\left|\frac{u_{\varepsilon}(\varepsilon i)-u_{\varepsilon}(\varepsilon(i-1))}{\varepsilon}\right|^{2}
\end{aligned}
$$


(the inequality is obtained by minimizing over all functions $w$ with $w(\varepsilon(i-1))=u_{\varepsilon}(\varepsilon i)$ and $w(\varepsilon(i-1))=u_{\varepsilon}(\varepsilon i)$; the minimum problem in the second line is obtained by scaling such $w$ and using the periodicity of $a$, the third line is a direct computation of the previous minimum). If we define $\widetilde{u}_{\varepsilon}$ as the piecewise-affine interpolation of $u_{\varepsilon}$ on $\varepsilon \mathbb{Z}$ then the estimate above shows that

$$
F_{\varepsilon}\left(u_{\varepsilon}\right) \geq F_{\text {hom }}\left(\widetilde{u}_{\varepsilon}\right) .
$$

The functional on the right-hand side is independent of $\varepsilon$ and with a convex integrand; hence, it is lower semicontinuous with respect to the weak $H^{1}$-convergence. Since $\widetilde{u}_{\varepsilon} \rightarrow u$ we then deduce

$$
\liminf _{\varepsilon \rightarrow 0} F_{\varepsilon}\left(u_{\varepsilon}\right) \geq \liminf _{\varepsilon \rightarrow 0} F_{\text {hom }}\left(\widetilde{u}_{\varepsilon}\right) \geq F_{\text {hom }}(u)
$$

i.e., the liminf inequality. The ansatz for the upper bound is obtained by optimizing the lower bound: recovery sequences oscillate around the target function in an optimal way. If the target function is linear (or affine) $u(x)=z x$ then a recovery sequence is obtained by taking the 1-periodic function $v$ minimizing

$$
\min \left\{\int_{0}^{1} a(y)\left|v^{\prime}+1\right|^{2} d y: v(0)=v(1)=0\right\}=\underline{a}
$$

and setting

$$
u_{\varepsilon}(x)=z\left(x+\varepsilon v\left(\frac{x}{\varepsilon}\right)\right) .
$$

This construction can be repeated up to a small error if $u$ is piecewise affine, and then carries over to arbitrary $u$ by density.

As a particular case we can fix $\theta \in[0,1]$ and consider the 1 -periodic $a$ given on $[0,1)$ by

$$
a(y)= \begin{cases}\alpha & \text { if } 0 \leq y<\theta \\ \beta & \text { if } \theta \leq y<1\end{cases}
$$

In this case we have

$$
\underline{a}=\frac{\alpha \beta}{\theta \beta+(1-\theta) \alpha} .
$$

Note that the same result is obtained only assuming that $|\{y \in(0,1): a(y)=\alpha\}|=\theta$ and $|\{y \in(0,1): a(y)=\beta\}|=1-\theta$. Thus in one dimension the limit depends only on the volume fraction of $\alpha$.

In the higher-dimensional case the limit can still be described by an elliptic integral, of the form

$$
F_{\mathrm{hom}}(u)=\int_{\Omega}\langle A \nabla u, \nabla u\rangle d x
$$

where $A$ is a constant symmetric matrix with $\underline{a} I \leq A \leq \bar{a} I$ ( $I$ the identity matrix) with strict inequalities unless $a$ is constant. If we take in two dimensions $a\left(y_{1}, y_{2}\right)=a\left(y_{1}\right)$ (a laminate in the first direction) then $A$ is a diagonal matrix with $\operatorname{diag}(\underline{a}, \bar{a})$. Of course, if 
$a\left(y_{1}, y_{2}\right)=a\left(y_{2}\right)$ then the two values are interchanged. If $a$ takes only the values $\alpha$ and $\beta$ in particular this shows that in the higher-dimensional case the results depends on the geometry of $\{y \in(0,1): a(y)=\alpha\}$ (often referred to as the microgeometry of the problem) and not only on the volume fraction.

In order to make minimum problems meaningful, we may consider the affine space $X=\varphi+H_{0}^{1}(\Omega)$ (i.e., we consider only functions with $u=\varphi$ on $\partial \Omega$ ). It can be proved that this boundary condition is 'compatible' with the $\Gamma$-limit; i.e., that the $\Gamma$-limit is the restriction to $X$ of the previous one, or, equivalently that recovery sequences for the first $\Gamma$-limit can be taken satisfying the same boundary data as their limit. As a consequence of Thorem 1.3.1 we then conclude that oscillating minimum problems for $F_{\varepsilon}$ with fixed boundary data are approximated by a simpler minimum problem with the same boundary data. Note however that all energies, both $F_{\varepsilon}$ and $F_{\text {hom }}$, are strictly convex, which implies that they have no local non global minimizer.

Example 1.4.2 We can add some continuously converging perturbation to obtain some more convergence result. For example, we can add perturbations of the form

$$
G_{\varepsilon}(u)=\int_{\Omega} g\left(\frac{x}{\varepsilon}, u\right) d x .
$$

On $g$ we make the following hypothesis:

$g$ is a Borel function 1-periodic in the first variable and uniformly Lipschitz in the second one; i.e.,

$$
\left|g(y, z)-g\left(y, z^{\prime}\right)\right| \leq L\left|z-z^{\prime}\right|
$$

We then have a perturbed homogenization result as follows.

Proposition 1.4.3 The functionals $F_{\varepsilon}+G_{\varepsilon} \Gamma$-converge both in the $L^{1}$ topology to the functional $F_{\text {hom }}+G$, where

$$
G(u)=\int_{\Omega} \bar{g}(u) d x, \quad \text { and } \quad \bar{g}(z)=\int_{(0,1)^{n}} g(y, z) d y
$$

is simply the average of $g(\cdot, z)$.

Proof. By Proposition 1.2.4 it suffices to show that $G_{\varepsilon}$ converges continuously with respect to the $L^{1}$-convergence. If $u_{\varepsilon} \rightarrow u$ in $L^{1}$ then

$$
\begin{aligned}
\left|G_{\varepsilon}\left(u_{\varepsilon}\right)-G(u)\right| & \leq \int_{\Omega}\left|g\left(\frac{x}{\varepsilon}, u_{\varepsilon}\right)-g\left(\frac{x}{\varepsilon}, u\right)\right| d x+\left|G_{\varepsilon}(u)-G(u)\right| \\
& \leq L \int_{\Omega}\left|u_{\varepsilon}-u\right| d x+\left|G_{\varepsilon}(u)-G(u)\right| .
\end{aligned}
$$

It suffices then to show that $G_{\varepsilon}$ converges pointwise to $G$. If $u$ is piecewise constant then this follows immediately from the Riemann-Lebesgue Lemma. Noting that also $\left|\bar{g}(z)-\bar{g}\left(z^{\prime}\right)\right| \leq$ 
$L\left|z-z^{\prime}\right|$ we easily obtain the convergence for $u \in L^{1}(\Omega)$ by the density of piecewise-constant functions.

Note that with a slightly more technical proof we can improve the Lipschitz continuity condition to a local Lipschitz continuity of the form

$$
\left|g(y, z)-g\left(y, z^{\prime}\right)\right| \leq L\left(1+|z|+\left|z^{\prime}\right|\right)\left|z-z^{\prime}\right|
$$

In particular in $1 \mathrm{D}$ we can apply the result for $g(y, z)=a(y)|z|^{2}$ and we have that

$$
\int_{\Omega} a\left(\frac{x}{\varepsilon}\right)\left(\left|u^{\prime}\right|^{2}+|u|^{2}\right) d x
$$

$\Gamma$-converges to

$$
\int_{\Omega}\left(\underline{a}\left|u^{\prime}\right|^{2}+\bar{a}|u|^{2}\right) d x
$$

As a consequence of Theorem 1.3.1, under the condition of coerciveness

$$
\lim _{z \rightarrow \pm \infty} \inf g(\cdot, z)=+\infty
$$

we obtain a convergence result as follows.

Proposition 1.4.4 The solutions to the minimum problems

$$
\min \left\{F_{\varepsilon}(u)+G_{\varepsilon}(u): u \in H^{1}(\Omega)\right\}
$$

converge (up to subsequences) to a constant function $\bar{u}$, whose constant value minimizes $\bar{g}$.

Proof. The proof of the proposition follows immediately from Theorem 1.3.1, once we observe that by the coerciveness and continuity of $\bar{g}$ a minimizer for that function exists, and the constant function $\bar{u}$ defined above minimizes both $F_{\text {hom }}$ and $G$.

If $g$ is differentiable then by computing the Euler-Lagrange equations of $F_{\varepsilon}+G_{\varepsilon}$ we conclude that we obtain solutions of

$$
-\sum_{i j} \frac{\partial}{\partial x_{i}}\left(a\left(\frac{x}{\varepsilon}\right) \frac{\partial u_{\varepsilon}}{\partial x_{i}}\right)+\frac{\partial}{\partial u} g\left(\frac{x}{\varepsilon}, u_{\varepsilon}\right)=0
$$

with Neumann boundary conditions, converging to the constant $\bar{u}$. 


\subsection{Higher-order $\Gamma$-limits and a choice criterion}

If the hypotheses of Theorem 1.3.1 are satisfied then we have noticed that every minimum point of the limit $F$ corresponds to a minimizing sequence for $F_{\varepsilon}$. However, not all points may be limits of minimizers for $F_{\varepsilon}$, and it may be interesting to discriminate between limits of minimizing sequences with different speeds of convergence. To this end, we may look at scaled $\Gamma$-limits. If we suppose that, e.g., $u$ is a limit of a sequence $\left(u_{\varepsilon}\right)$ with

$$
F_{\varepsilon}\left(u_{\varepsilon}\right)=\min F+O\left(\varepsilon^{\alpha}\right)
$$

for some $\alpha>0$ (but, of course, the rate of convergence may also not be polynomial) then we may look at the $\Gamma$-limit of the scaled functionals

$$
F_{\varepsilon}^{\alpha}(u)=\frac{F_{\varepsilon}\left(u_{\varepsilon}\right)-\min F}{\varepsilon^{\alpha}} .
$$

Suppose that $F_{\varepsilon}^{\alpha} \Gamma$-converges to some $F^{\alpha}$ not taking the value $-\infty$. Then:

(i) the domain of $F^{\alpha}$ is contained in the set of minimizers of $F$ (but may as well be empty);

(ii) $F^{\alpha}(u) \neq+\infty$ if and only if there exists a recovery sequence for $u$ satisfying (1.16).

Moreover, we can apply Theorem 1.3.1 to $F_{\varepsilon}^{\alpha}$ and obtain the following result, which gives a choice criterion among minimizers of $F$.

Theorem 1.5.1 Let the hypotheses of Theorem 1.3.1 be satisfied and the functionals in (1.17) $\Gamma$-converge to some $F^{\alpha}$ not taking the value $-\infty$ and not identically $+\infty$. Then

(i) $\inf F_{\varepsilon}=\min F+\varepsilon^{\alpha} \min F^{\alpha}+o\left(\varepsilon^{\alpha}\right)$;

(ii) if $F_{\varepsilon}\left(u_{\varepsilon}\right)=\min F_{\varepsilon}+o\left(\varepsilon^{\alpha}\right)$ and $u_{\varepsilon} \rightarrow u$ then $u$ minimizes both $F$ and $F^{\alpha}$.

Proof. We can apply Theorem 1.3.1 to a (subsequence of a) converging minimizing sequence for $F_{\varepsilon}^{\alpha}$; i.e., a sequence satisfying hypothesis (ii). Its limit point $u$ satisfies

$$
F^{\alpha}(u)=\min F^{\alpha}=\lim _{\varepsilon \rightarrow 0} \min F_{\varepsilon}^{\alpha}=\lim _{\varepsilon \rightarrow 0} \frac{\min F_{\varepsilon}-\min F}{\varepsilon^{\alpha}}
$$

which proves (i). Since, as already remarked $u$ is also a minimizer of $F$, we also have (ii).

Example 1.5.2 Simple examples in the real line:

(1) if $F_{\varepsilon}(x)=\varepsilon x^{2}$ then $F(x)=0$. We have $F^{\alpha}(x)=0$ if $0<\alpha<1, F^{1}(x)=x^{2}$ (if $\alpha=1)$, and

$$
F^{\alpha}(x)= \begin{cases}0 & x=0 \\ +\infty & x \neq 0\end{cases}
$$

if $\alpha>1$; 
(2) if $F_{\varepsilon}(x)=\left(x^{2}-1\right)^{2}+\varepsilon(x-1)^{2}$ then $F(x)=\left(x^{2}-1\right)^{2}$. We have

$$
F^{\alpha}(x)= \begin{cases}0 & |x|=1 \\ +\infty & |x| \neq 1\end{cases}
$$

if $0<\alpha<1$,

$$
F^{1}(x)= \begin{cases}0 & x=1 \\ 4 & x=-1 \\ +\infty & |x| \neq 1\end{cases}
$$

if $\alpha=1$,

$$
F^{\alpha}(x)= \begin{cases}0 & x=1 \\ +\infty & x \neq 1\end{cases}
$$

if $\alpha>1$.

Remark 1.5.3 It must be observed that the functionals $F_{\varepsilon}^{\alpha}$ in Theorem 1.5.1 are often equicoercive with respect to a stronger topology than the original $F_{\varepsilon}$, so that we can improve the convergence in (ii).

\section{Example 1.5.4 (Gradient theory of phase transitions) Let}

$$
F_{\varepsilon}(u)=\int_{\Omega}\left(W(u)+\varepsilon^{2}|\nabla u|^{2}\right) d x
$$

be defined in $L^{1}(\Omega)$ with domain in $H^{1}(\Omega)$. Here $W(u)=\left(u^{2}-1\right)^{2}$ (or a more general double-well potential; i.e., a non-negative function vanishing exactly at \pm 1$)$. Then $\left(F_{\varepsilon}\right)$ is equicoercive with respect to the weak $L^{1}$-convergence. Since this convergence is metrizable on bounded sets, we can consider $L^{1}(\Omega)$ equipped with this convergence. The $\Gamma$-limit is then simply

$$
F^{0}(u)=\int_{\Omega} W^{* *}(u) d x
$$

where $W^{* *}$ is the convex envelope of $W$; i.e. $W^{* *}(u)=\left(\left(u^{2}-1\right) \vee 0\right)^{2}$. All functions with $\|u\|_{\infty} \leq 1$ are minimizers of $F^{0}$.

We take $\alpha=1$ and consider

$$
F_{\varepsilon}^{1}(u)=\int_{\Omega}\left(\frac{W(u)}{\varepsilon}+\varepsilon|\nabla u|^{2}\right) d x .
$$

Then $\left(F_{\varepsilon}^{1}\right)$ is equicoercive with respect to the strong $L^{1}$-convergence, and its $\Gamma$-limit is

$$
F^{1}(u)=c_{W} \mathcal{H}^{n-1}(\partial\{u=1\} \cap \Omega) \text { for } u \in B V(\Omega ;\{ \pm 1\}),
$$


and $+\infty$ otherwise, where $c_{W}=8 / 3$ (in general $c_{W}=2 \int_{-1}^{1} \sqrt{W(s)} d s$ ). This results states that recovery sequences $\left(u_{\varepsilon}\right)$ tend to sit in the bottom of the wells (i.e., $u \in \pm 1$ ) in order to make $\frac{W\left(u_{\varepsilon}\right)}{\varepsilon}$ finite; however, every 'phase transition' costs a positive amount, which is optimized by balancing the effects of the two terms in the integral. Indeed, by optimizing the interface between the phases $\{u=1\}$ and $\{u=-1\}$ one obtains the optimal 'surface tension' $c_{W}$.

In one dimension the ansatz on the recovery sequences around a jump point $x_{0}$ is that they are of the form

$$
u_{\varepsilon}(x)=v\left(\frac{x-x_{0}}{\varepsilon}\right)
$$

where $v$ minimizes

$$
\min \left\{\int_{-\infty}^{+\infty}\left(W(v)+\left|v^{\prime}\right|^{2}\right) d x: v( \pm \infty)= \pm 1\right\}=2 \int_{-1}^{1} \sqrt{W(s)} d s .
$$

In more than one dimension the ansatz becomes

$$
u_{\varepsilon}(x)=v\left(\frac{d(x,\{u=1\})}{\varepsilon}\right),
$$

where $d(\cdot, A)$ is the signed distance from the set $A$. This means that around the interface $\partial\{u=1\}$ the recovery sequence pass from -1 to +1 following the one-dimensional profile of $v$ essentially on a $O(\varepsilon)$-neighbourhood of $\partial\{u=1\}$.

Note that

(i) we have an improved convergence of recovery sequences from weak to strong $L^{1}$ convergence;

(ii) the domain of $F^{1}$ is almost disjoint from that of the $F_{\varepsilon}^{1}$, the only two functions in common being the constants \pm 1 ;

(iii) in order to make the $\Gamma$-limit properly defined we have to use the space of functions of bounded variation or, equivalently, the family of sets of finite perimeter if we take as parameter the set $A=\{u=1\}$. In this context the set $\partial\{u=1\}$ is properly defined in a measure-theoretical way, as well as its $(n-1)$-dimensional Hausdorff measure.

Example 1.5.5 (linearized fracture mechanics from interatomic potentials) We now give an example in which the scaling of the variable, and not only of the energy is part of the problem. We consider a systems of one-dimensional nearest-neighbour atomistic interactions through a Lennard-Jones type interaction. Note that by the one-dimensional nature of the problem we can parameterize the position of the atoms as an increasing function of the parameter.

Let $\psi$ be a $C^{2}$ potential as in Figure 1.1, with domain $(0,+\infty)$ (we set $\psi(z)=+\infty$ for $z \leq 0)$, minimum in 1 with $\psi^{\prime \prime}(1)>0$, convex in $\left(0, z_{0}\right)$, concave in $\left(z_{0},+\infty\right)$ and tending to $\psi(\infty)<+\infty$ at $+\infty$. A possible choice is Lennard Jones potential

$$
\psi(z)=\frac{1}{z^{12}}-\frac{2}{z^{6}} .
$$




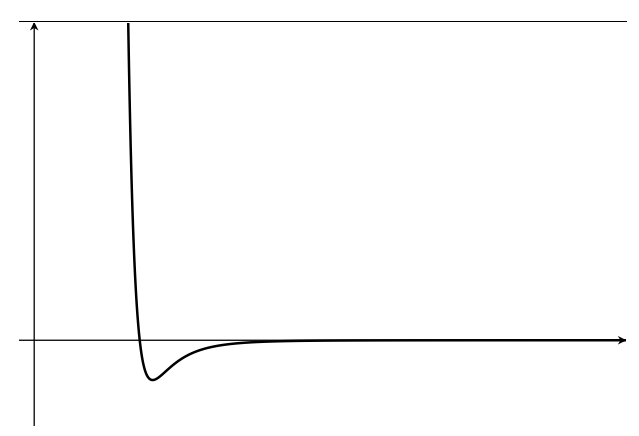

Figure 1.1: a Lennard-Jones potential

We consider the energy

$$
\Psi_{N}(v)=\sum_{i=1}^{N} \psi\left(v_{i}-v_{i-1}\right)
$$

with $N \in \mathbb{N}$ defined on $v_{i}$ with $v_{i}>v_{i-1}$. We introduce the small parameter $\varepsilon=1 / N$ and identify the vector $\left(v_{0}, \ldots, v_{N}\right)$ is identified with a discrete function defined on $\varepsilon \mathbb{Z} \cap[0,1]$. A non-trivial $\Gamma$-limit will be obtained by scaling and rewriting the energy in terms of a scaled variable

$$
u=\sqrt{\varepsilon}\left(v-\frac{i d}{\varepsilon}\right)
$$

i.e., $u_{i}=\sqrt{\varepsilon}\left(v_{i}-i\right)$. This scaling can be justified noting that (up to additive constants) $v_{i}=i=i d / \varepsilon$ is the absolute minimum of the energy. The scaled energies that we consider are

$$
F_{\varepsilon}(u)=\Psi_{N}\left(\frac{i d}{\varepsilon}+\frac{u}{\sqrt{\varepsilon}}\right)-\min \Psi_{N}=\sum_{i=1}^{N} J\left(\frac{u_{i}-u_{i-1}}{\sqrt{\varepsilon}}\right),
$$

where

$$
J(w)=\psi(1+w)-\min \psi=\psi(1+w)-\psi(1) .
$$

For convenience we extend the function to all $\mathbb{R}$ setting $J(w)=+\infty$ if $w \leq 0$. Again, the vector $\left(u_{0}, \ldots, u_{N}\right)$ is identified with a discrete function defined on $\varepsilon \mathbb{Z} \cap[0,1]$ or with its piecewise-affine interpolation. With this last identification, $F_{\varepsilon}$ can be viewed as functionals in $L^{1}(0,1)$, and their $\Gamma$-limit computed with respect to that topology.

We denote $w_{0}=1+z_{0}$. It must be noted that for all $\bar{w}>0$ we have

$$
\#\left\{i: \frac{u_{i}-u_{i-1}}{\sqrt{\varepsilon}}>\bar{w}\right\} \leq \frac{1}{J(\bar{w})} F_{\varepsilon}(u),
$$

so that this number of indices is equi-bounded along sequences with equibounded energy. We may therefore suppose that the corresponding points $\varepsilon i$ converge to a finite set $S \subset$ 
$[0,1]$. For fixed $\bar{w}$, we have $J(w) \geq \bar{c}|w|^{2}$ on $(-\infty, \bar{w}]$ for some $\bar{c}>0$; this gives, if $A$ is compactly contained in $(0,1) \backslash S$, that

$$
F_{\varepsilon}(u) \geq \bar{c} \sum_{i}\left(\frac{u_{i}-u_{i-1}}{\sqrt{\varepsilon}}\right)^{2}=\bar{c} \sum_{i} \varepsilon\left(\frac{u_{i}-u_{i-1}}{\varepsilon}\right)^{2} \geq \bar{c} \int_{A}\left|u^{\prime}\right|^{2} d t
$$

(the sum extended to $i$ such that $\frac{u_{i}-u_{i-1}}{\sqrt{\varepsilon}} \leq \bar{w}$ ). By the arbitrariness of $A$ in this estimate we then have that if $u_{\varepsilon} \rightarrow u$ and $F_{\varepsilon}\left(u_{\varepsilon}\right) \leq C<+\infty$ then $u$ is piecewise- $H^{1}$; i.e., there exists a finite set $S \subset(0,1)$ such that $u \in H^{1}((0,1) \backslash S)$; we denote by $S(u)$ the minimal set such that $u \in H^{1}((0,1) \backslash S(u))$. The reasoning above also shows that

$$
\bar{c} \int_{0}^{1}\left|u^{\prime}\right|^{2} d t+J(\bar{w}) \#(S(u))
$$

is a lower bound for the $\Gamma$-limit of $F_{\varepsilon}$. The $\Gamma$-limit on piecewise- $H^{1}(0,1)$ functions can be computed by optimizing the choice of $\bar{w}$ and $\bar{c}$, obtaining

$$
F(u)=\frac{1}{2} J^{\prime \prime}(0) \int_{0}^{1}\left|u^{\prime}\right|^{2} d t+J(\infty) \#(S(u))
$$

with the constraint that $u^{+}>u^{-}$on $S(u)$. This functional is the one-dimensional version of Griffith's fracture energy for brittle materials, and coincides with a functional introduced by Mumford-Shah in the framework of Image Reconstruction (without the constraint $u^{+}>$ $\left.u^{-}\right)$.

Note that the parameterization of $v_{i}$ on $\varepsilon \mathbb{Z}$ would suggest to interpret $v_{i}-v_{i-1}$ as a different quotient and hence the change of variables $u_{i}=\varepsilon v_{i}-i d$. This would give an energy of the form

$$
\widetilde{F}_{\varepsilon}(u)=\sum_{i=1}^{N} J\left(\frac{u_{i}-u_{i-1}}{\varepsilon}\right)
$$

it can be shown that $\widetilde{F}_{\varepsilon}$ converges to the energy with domain the set of piecewise-affine increasing $u$ with $u^{\prime}=1$ a.e., and for such $u$

$$
\widetilde{F}(u)=J(\infty) \#(S(u))
$$

This different choice of the parameterization hence only captures the fracture part of the energy.

\subsection{References to Chapter 1}

For an introduction to $\Gamma$-convergence we refer to the 'elementary' book [9]. More examples, and an overview of the methods for the computation of $\Gamma$-limits can be found in [11]. More detailed information on topological properties of $\Gamma$-convergence are found in [21]. Homogenization results are described in [13]. 


\section{Chapter 2}

\section{Parameterized motion driven by global minimization}

Even though dynamic variational problems are in general associated with a local minimization procedure, for 'slow movements' the notion of a 'quasi-static' motion can be defined starting from a global-minimization criterion. The ingredients are

- a parameter-dependent energy;

- a dissipation satisfying a non-decreasing constraint;

- (time-)parameterized forcing condition.

Loosely speaking, a quasi static motion derives from some parameterized forcing condition (applied forces, varying boundary conditions or other constraints); the motion is thought to be so slow so that the solution at a fixed value of the parameter (at fixed 'time') minimizes a total energy. This energy is obtained adding some 'dissipation' to some 'internal energy'. A further condition is that the dissipation increases with time. An entire general theory (of rate-independent motion) can be developed starting from these ingredients.

\subsection{Damage models}

In this section we deal with a simplified example, with the aim of examining its stability with respect to perturbations.

\subsubsection{Damage of a homogeneous material}

We consider a one-dimensional setting. Our functions will be parameterized on a fixed interval $(0,1)$. In this case we have

- the parameter space will be that of all measurable subsets $A$ of $(0,1)$. The set $A$ will be understood as the damage set; 
- the energies depending on a set $A$ will be

$$
F_{A}(u)=\alpha \int_{A}\left|u^{\prime}\right|^{2} d x+\beta \int_{(0,1) \backslash A}\left|u^{\prime}\right|^{2} d x,
$$

where $0<\alpha<\beta$. In an mechanical interpretation of the variables, $u$ represents the deformation of a bar, whose elastic constant is $\beta$ in the undamaged set and $\alpha<\beta$ in the damaged set;

- the dissipation is

$$
D(A)=\gamma|A|,
$$

with $\gamma>0$. The work done to damage a portion $A$ of the material is proportional to the measure of $A$;

- the condition that forces the solution to be parameter dependent ('time-dependent') is a boundary condition

$$
u(0)=0, \quad u(1)=g(t),
$$

where $g$ is a continuous function with $g(0)=0$. Here the parameter is $t \in[0, T]$.

Definition 2.1.1 $A$ solution to the evolution related to the energy, dissipation and boundary conditions above is a pair $\left(u^{t}, A^{t}\right)$ with $u^{t} \in H^{1}(0,1), A^{t} \subset(0,1)$, and such that

- (monotonicity) we have $A^{s} \subset A^{t}$ for all $s<t$

- (minimization) the pair $\left(u^{t}, A^{t}\right)$ minimizes

$$
\min \left\{F_{A}(u)+D(A): u(0)=0, u(1)=g(t), A^{t} \subset A\right\}
$$

- continuity the energy $\mathcal{E}(t)=F_{A^{t}}\left(u^{t}\right)+D\left(A^{t}\right)$ is continuous

- homogeneous initial datum $u^{0}$ is the constant 0 and $A^{0}=\emptyset$.

The continuity assumption allows to rule out trivial solutions as those with $A^{t}=(0,1)$ for all $t>0$. It is usually replaced by a more physical condition of energy conservation. In our context this assumption is not relevant.

Note that $t$ acts only as a parameter (the motion is 'rate independent'). Hence, for example if $g$ is monotone increasing, it suffices to consider $g(t)=t$. We will construct by hand a solution in this simplified one-dimensional context.

Remark 2.1.2 Note that the value in the minimum problem

$$
m(t)=\min \left\{F_{A}(u)+D(A): u(0)=0, u(1)=t\right\}
$$

depends on $A$ only through $\lambda=|A|$.

In fact, given $A$, we can examine the minimum problem

$$
m(A, t)=\min \left\{\int_{A} \alpha\left|u^{\prime}\right|^{2} d x+\beta \int_{(0,1) \backslash A} \beta\left|u^{\prime}\right|^{2} d x: u(0)=0, u(1)=t\right\} .
$$


For all test function $u$ we have, by Jensen's inequality

$$
\int_{A} \alpha\left|u^{\prime}\right|^{2} d x+\beta \int_{(0,1) \backslash A} \beta\left|u^{\prime}\right|^{2} d x \geq\left.\alpha|A| z_{1}\right|^{2}+\beta(1-|A|)\left|z_{2}\right|^{2},
$$

Where

$$
z_{1}=\frac{1}{|A|} \int_{A} u^{\prime} d x, \quad z_{2}=\frac{1}{1-|A|} \int_{(0,1) \backslash A} u^{\prime} d x
$$

with a strict inequality if $u^{\prime}$ is not constant on $A$ and $(0,1) \backslash A$. This shows that the unique minimizer satisfies

$$
u^{\prime}=z_{1} \chi_{A}+z_{2}\left(1-\chi_{A}\right), \quad|A| z_{1}+(1-|A|) z_{2}=t,
$$

where the second condition is given by the boundary data. Hence

$$
m(A, t)=\min \left\{\alpha \lambda\left|z_{1}\right|^{2}+\beta(1-\lambda)\left|z_{2}\right|^{2}: \lambda z_{1}+(1-\lambda) z_{2}=t\right\}=\frac{\alpha \beta}{\lambda \beta+(1-\lambda) \alpha} t^{2} .
$$

We conclude that the minimum value $(2.2)$ is given by

$$
\frac{\alpha \beta}{\lambda \beta+(1-\lambda) \alpha} t^{2}+\gamma \lambda
$$

By minimizing over $\lambda$ we obtain the optimal value of the measure of the damaged region

$$
\lambda_{\min }(t)= \begin{cases}0 & \text { if }|t| \leq \sqrt{\frac{\alpha \gamma}{\beta(\beta-\alpha)}} \\ 1 & \text { if }|t| \geq \sqrt{\frac{\beta \gamma}{\alpha(\beta-\alpha)}} \\ t \sqrt{\frac{\alpha \beta}{\gamma(\beta-\alpha)}}-\frac{\alpha}{\beta-\alpha} & \text { otherwise }\end{cases}
$$

and the minimum value

$$
m(t)= \begin{cases}\beta t^{2} & \text { if }|t| \leq \sqrt{\frac{\alpha \gamma}{\beta(\beta-\alpha)}} \\ \alpha t^{2}+\gamma & \text { if }|t| \geq \sqrt{\frac{\beta \gamma}{\alpha(\beta-\alpha)}} \\ 2 t \sqrt{\frac{\alpha \beta \gamma}{\beta-\alpha}}-\frac{\gamma \alpha}{\beta-\alpha} & \text { otherwise. }\end{cases}
$$

The interpretation of this formula is as follows. For small values of the total displacement $t$ the material remains undamaged, until it reaches a critical value for the boundary datum. Then a portion of size $\lambda_{\min }(t)$ of the material damages, lowering the elastic constant of the 
material and the overall value of the sum of the internal energy and the dissipation, until all the material is damaged. Note that in this case $\mathcal{E}(t)=m(t)$.

The solutions for the evolution problem are given by any increasing family of sets $A^{t}$ satisfying $\left|A^{t}\right|=\lambda_{\min }(t)$ and correspondingly functions $u^{t}$ minimizing $m\left(A^{t}, t\right)$.

The value in (2.3) is obtained by first minimizing in $u$. Conversely, we may first minimize in $A$. We then have

$$
\min \left\{\int_{0}^{1} \min _{A}\left\{\chi_{A}\left(\alpha\left|u^{\prime}\right|^{2}+\gamma\right), \chi_{(0,1) \backslash A} \beta\left|u^{\prime}\right|^{2}\right\} d x: u(0)=0, u(1)=g(t)\right\}
$$

The lower-semicontinuous envelope of the integral energy is given by the integral with energy function the convex envelope of

$$
f(z)=\min \left\{\alpha z^{2}+\gamma, \beta z^{2}\right\}
$$

which is exactly given by formula (2.5); i.e.,

$$
m(t)=f^{* *}(t)
$$

(see Fig. 2.1)

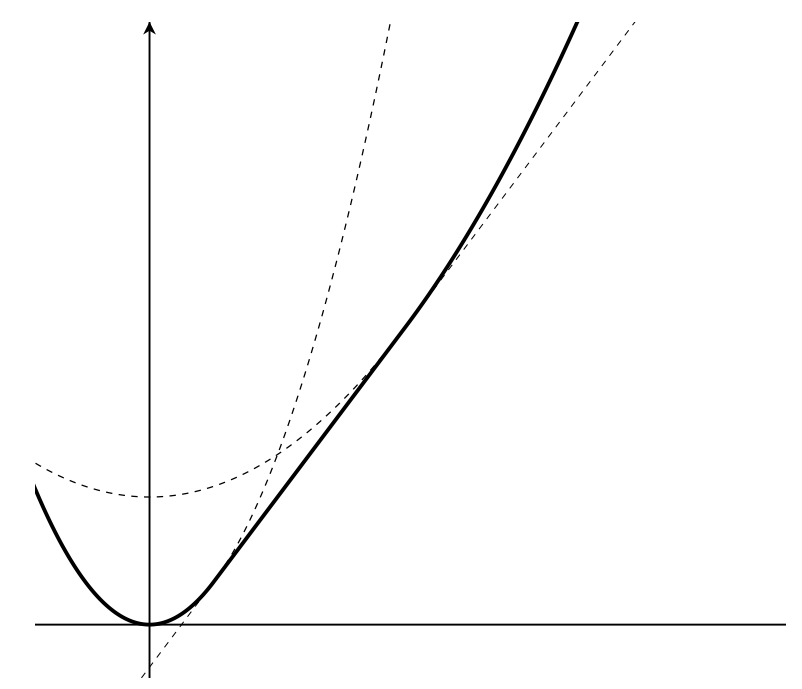

Figure 2.1: minimal value $m(t)$ for the damage problem

Irreversibility. An important feature of the monotonicity condition for $A^{t}$ is irreversibility of damage, which implies that for non-increasing $g$ the values of $m(g(t))$ will 
depend on the highest value taken by $\lambda_{\min }(g(t))$ on $[0, t]$. In particular, for a 'loadingunloading' cycle with $g(t)=\frac{T}{2}-\left|t-\frac{T}{2}\right|$, the value of $\mathcal{E}(t)$ is given by

$$
\mathcal{E}(t)= \begin{cases}m(t) & \text { for } 0 \leq t \leq T / 2 \\ \frac{\alpha \beta}{\lambda_{\min }(T / 2) \beta+\left(1-\lambda_{\min }(T / 2)\right) \alpha}(T-t)^{2}+\gamma \lambda_{\min }(T / 2) & \text { for } T / 2 \leq t \leq T .\end{cases}
$$

This formula highlights that once the maximal value $\lambda_{\min }(T / 2)$ is reached, then the damaged region $A^{t}$ remains fixed, so that the problem becomes a quadratic minimization (plus the constant value of the dissipation). We plot $m^{\prime}(t)$ and draw a cycle in Fig. 2.2

In particular, if $\frac{T}{2} \geq \sqrt{\frac{\beta \gamma}{\alpha(\beta-\alpha)}}$ then the material is completely damaged in the 'unloading' regime.

\section{Threshold formulation}

Note that a solution $u_{t}$ of (2.2) satisfies the Euler-Lagrance equation

$$
\left(\left(\alpha \chi_{A}+\beta\left(1-\chi_{A}\right)\right) u^{\prime}\right)^{\prime}=0
$$

i.e.,

$$
\left(\alpha \chi_{A}+\beta\left(1-\chi_{A}\right)\right) u^{\prime}=\sigma_{t},
$$

where $\sigma_{t}$ is a constant parameterized by $t$. Its plot as a function of $g=g(t)$ along a 'loading-unloading' cycle is given in Fig. 2.2.

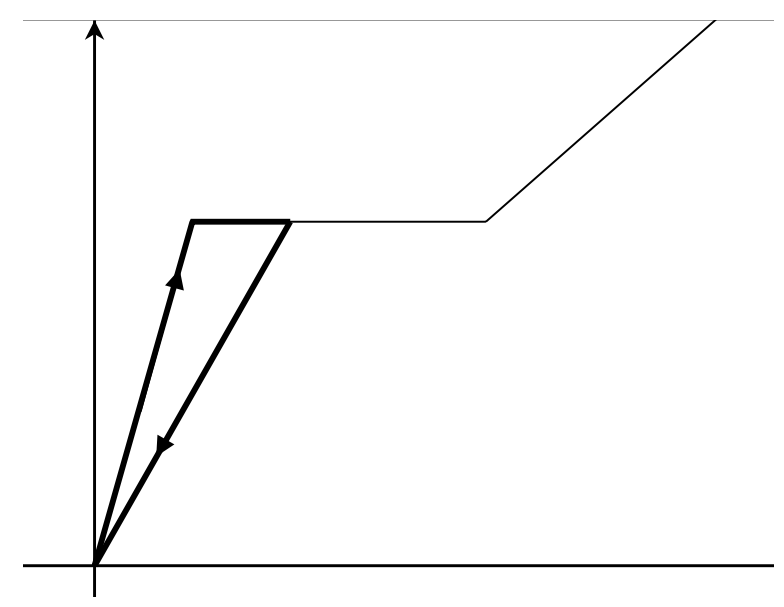

Figure 2.2: plot of $\sigma_{t}$ along a cycle 
The plateau for $\sigma$ is obtained at the threshold value

$$
\sigma=\sqrt{\frac{\alpha \beta \gamma}{\beta-\alpha}} .
$$

We can interpret the $g-\sigma$ graph as a threshold phenomenon: the material does not damage until the stress $\sigma$ reaches the threshold value. At this point, if the material is loaded further it damages so as to keep the value of $\sigma$ below the threshold, until all the material is damaged. If the material is unloaded then $\sigma$ follows a linear elastic behavior with the overall effective elastic constant corresponding to the total amount of damage produced.

\subsubsection{Homogenization of damage}

We now examine the behaviour of the previous process with respect to $\Gamma$-convergence in the case of homogenization. To that end we introduce the energies

$$
F_{\varepsilon, A}(u)=\int_{(0,1) \backslash A} \beta\left(\frac{x}{\varepsilon}\right)\left|u^{\prime}\right|^{2} d x+\int_{A} \alpha\left(\frac{x}{\varepsilon}\right)\left|u^{\prime}\right|^{2} d x
$$

where $\alpha$ and $\beta$ are 1-periodic functions with

$$
\alpha(y)=\left\{\begin{array}{ll}
\alpha_{1} & \text { for } 0 \leq y<\frac{1}{2} \\
\alpha_{2} & \text { for } \frac{1}{2} \leq y<1
\end{array} \quad \beta(y)= \begin{cases}\beta_{1} & \text { for } 0 \leq y<\frac{1}{2} \\
\beta_{2} & \text { for } \frac{1}{2} \leq y<1\end{cases}\right.
$$

with $0<\alpha_{j}<\beta_{j}$. Note that for fixed $A$ the functionals $F_{\varepsilon, A} \Gamma$-converge to

$$
F_{\mathrm{hom}, A}(u)=\underline{\beta} \int_{(0,1) \backslash A}\left|u^{\prime}\right|^{2} d x+\underline{\alpha} \int_{A}\left|u^{\prime}\right|^{2} d x
$$

with

$$
\underline{\alpha}=\frac{2 \alpha_{1} \alpha_{2}}{\alpha_{1}+\alpha_{2}}<\frac{2 \beta_{1} \beta_{2}}{\beta_{1}+\beta_{2}}=\underline{\beta} .
$$

This can be easily checked if $A$ is an interval (or a union of intervals), and then for a general $A$ by approximation. Indeed if $A=(0, \lambda)$ then the liminf inequality trivially holds by separately applying the liminf inequality to the two energies

$$
\int_{0}^{\lambda} \alpha\left(\frac{x}{\varepsilon}\right)\left|u^{\prime}\right|^{2} d x, \quad \int_{\lambda}^{1} \beta\left(\frac{x}{\varepsilon}\right)\left|u^{\prime}\right|^{2} d x .
$$

Conversely, given a target function $u \in H^{1}(0,1)$ we can find recovery sequences $\left(u_{\varepsilon}^{1}\right)$ and $\left(u_{\varepsilon}^{2}\right)$ for $u$ on $(0, \lambda)$ and $(\lambda, 1)$, respectively, for the energies $(2.11)$ with $u_{\varepsilon}^{1}(\lambda)=u_{\varepsilon}^{2}(\lambda)$, so that the corresponding $u_{\varepsilon}$ defined as $u_{\varepsilon}^{1}$ on $(0, \lambda)$ and as $u_{\varepsilon}^{2}$ on $(\lambda, 1)$ is a recovery sequence 
for $F_{\text {hom, } A}(u)$. Note that the $\Gamma$-limit is still of the form examined above with constant $\alpha$ and $\beta$.

We now examine instead the damage process at fixed $\varepsilon$. For simplicity of computation we suppose that $\frac{1}{\varepsilon} \in \mathbb{N}$. The general case can be always reduced to this assumption up to an error of order $\varepsilon$. The dissipation will be of the form

$$
D_{\varepsilon}(A)=\int_{A} \gamma\left(\frac{x}{\varepsilon}\right) d x
$$

where again $\gamma$ is a 1-periodic function with

$$
\gamma(y)= \begin{cases}\gamma_{1} & \text { for } 0 \leq y<\frac{1}{2} \\ \gamma_{2} & \text { for } \frac{1}{2} \leq y<1\end{cases}
$$

with $\gamma_{j}>0$. In the case $\gamma_{1}=\gamma_{2}$ we obtain the same dissipation as above, independent of $\varepsilon$.

In order to compute the minimum value

$$
m^{\varepsilon}(t)=\min \left\{F_{\varepsilon, A}(u)+D_{\varepsilon}(A): u(0)=0, u(1)=t, A \subset(0,1)\right\}
$$

we proceed as in Remark 2.1.2, noticing that the minimum value

$$
m^{\varepsilon}(A, t)=\min \left\{F_{\varepsilon, A}(u): u(0)=0, u(1)=t\right\}
$$

depends on $A$ only through the volume fraction of each damaged component

$$
\lambda_{i}=2\left|\left\{x \in A: \alpha\left(\frac{x}{\varepsilon}\right)=\alpha_{i}\right\}\right| \text {. }
$$

and its value is independent of $\varepsilon$ and is given given by

$$
\begin{gathered}
\min \left\{\frac{1}{2}\left(\lambda_{1} \alpha_{1} z_{11}^{2}+\left(1-\lambda_{1}\right) \beta_{1} z_{12}^{2}\right)+\frac{1}{2}\left(\lambda_{2} \alpha_{2} z_{21}^{2}+\left(1-\lambda_{2}\right) \beta_{1} z_{22}^{2}\right):\right. \\
\left.\frac{1}{2}\left(\lambda_{1} z_{11}+\left(1-\lambda_{1}\right) z_{12}\right)+\frac{1}{2}\left(\lambda_{2} z_{21}+\left(1-\lambda_{2}\right) z_{22}\right)=t\right\}
\end{gathered}
$$

We conclude that $m^{\varepsilon}(t)=m_{\text {hom }}(t)$ is independent of $\varepsilon$ and satisfies

$$
m_{\mathrm{hom}}(t)=\frac{1}{2} \min \left\{m_{1}\left(t_{1}\right)+m_{2}\left(t_{2}\right): \frac{t_{1}+t_{2}}{2}=t\right\},
$$

where $m_{j}$ is defined as $m$ in (2.2) with $\alpha_{j}, \beta_{j}$ and $\gamma_{j}$ in the place of $\alpha, \beta$ and $\gamma$ (i.e., by the damage process in the $i$-th material). Hence, by (2.5)

$$
m_{j}(t)= \begin{cases}\beta_{j} t^{2} & \text { if }|t| \leq \sqrt{\frac{\alpha_{j} \gamma_{j}}{\beta_{j}\left(\beta_{j}-\alpha_{j}\right)}} \\ \alpha_{j} t^{2}+\gamma_{j} & \text { if }|t| \geq \sqrt{\frac{\beta_{j} \gamma_{j}}{\alpha_{j}\left(\beta_{j}-\alpha_{j}\right)}} \\ 2 t \sqrt{\frac{\alpha_{j} \beta_{j} \gamma_{j}}{\beta_{j}-\alpha_{j}}}-\frac{\gamma_{j} \alpha_{j}}{\beta_{j}-\alpha_{j}} & \text { otherwise. }\end{cases}
$$


We can therefore easily compute $m(t)$. In the hypothesis, e.g, that

$$
p_{2}:=\sqrt{\frac{\alpha_{2} \beta_{2} \gamma_{2}}{\beta_{2}-\alpha_{2}}}<\sqrt{\frac{\alpha_{1} \beta_{1} \gamma_{1}}{\beta_{1}-\alpha_{1}}}=: p_{1}
$$

we can write $m^{\prime}(t)$ as

$$
m_{\mathrm{hom}}^{\prime}(t)= \begin{cases}2 \underline{\beta} t & \text { if }|t| \leq \frac{p_{2}}{\underline{\beta}} \\ 2 p_{2} & \text { if } \frac{p_{2}}{\underline{\beta}}<|t|<\frac{p_{2}\left(\beta_{1}+\alpha_{2}\right)}{2 \beta_{1} \alpha_{2}} \\ \frac{4 \beta_{1} \alpha_{2}}{\beta_{1}+\alpha_{2}} t & \text { if } \frac{p_{2}\left(\beta_{1}+\alpha_{2}\right)}{2 \beta_{1} \alpha_{2}} \leq|t| \leq \frac{p_{1}\left(\beta_{1}+\alpha_{2}\right)}{2 \beta_{1} \alpha_{2}} \\ 2 p_{1} & \text { if } \frac{p_{1}\left(\beta_{1}+\alpha_{2}\right)}{2 \beta_{1} \alpha_{2}}<|t|<\frac{p_{1}}{\underline{\alpha}} \\ 2 \underline{\alpha} t & \text { if }|t| \geq \frac{p_{1}}{\underline{\alpha}} .\end{cases}
$$

The outcome is pictured in Fig. 2.3. It highlights that the behaviour is different from the

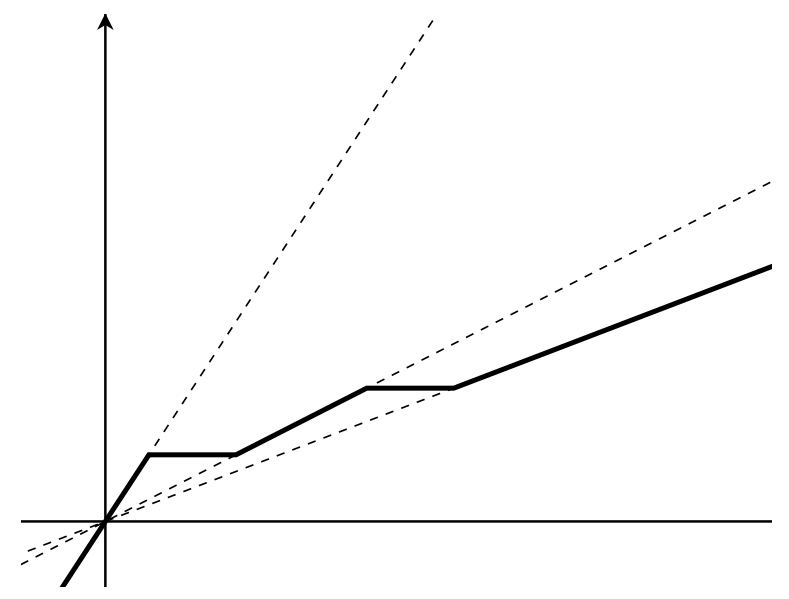

Figure 2.3: homogenized damage in a periodic microstructure

one computed above: for small values of the total displacement $t$ the overall response is the same as the one of the homogenized behaviour of the two 'strong' materials. At a first critical value one of the two materials (and only one except for the exceptional case $p_{1}=p_{2}$ ) starts to damage (this corresponds to the first constant value $2 p_{1}$ of $m^{\prime}$ ) until it is completely damaged. With the condition (2.16) the first material to damage is material 
2 , and the corresponding damage volume fraction is

$$
\lambda_{2, \min }(t)= \begin{cases}0 & \text { if } 0 \leq t \leq \frac{p_{2}}{\underline{\beta}} \\ \frac{2 p_{2}}{\gamma_{2}}\left(t-\frac{p_{2}}{\underline{\beta}}\right) & \text { if } \frac{p_{2}}{\underline{\beta}}<t<\frac{p_{2}\left(\beta_{1}+\alpha_{2}\right)}{2 \beta_{1} \alpha_{2}} \\ 1 & \text { if } t>\frac{p_{2}\left(\beta_{1}+\alpha_{2}\right)}{2 \beta_{1} \alpha_{2}}\end{cases}
$$

Then the material behaves as a mixture of a strong material 1 and a damaged material 2 . Subsequently, also material 1 starts to damage; the corresponding damage volume fraction is

$$
\lambda_{1, \min }(t)= \begin{cases}0 & \text { if } t \leq \frac{p_{1}\left(\beta_{1}+\alpha_{2}\right)}{2 \beta_{1} \alpha_{2}} \\ \frac{2 p_{1}}{\gamma_{1}}\left(t-\frac{p_{1}\left(\beta_{1}+\alpha_{2}\right)}{2 \beta_{1} \alpha_{2}}\right) & \text { if } \frac{p_{1}\left(\beta_{1}+\alpha_{2}\right)}{2 \beta_{1} \alpha_{2}}<t<\frac{p_{1}}{\underline{\alpha}} \\ 1 & \text { if } t \geq \frac{p_{1}}{\underline{\alpha}}\end{cases}
$$

After also material 1 has completely damaged, the behaviour is that of the homogenized energy for two weak materials.

Note that at fixed $\varepsilon$ we can define $A_{\varepsilon}^{t}$ and $u_{\varepsilon}^{t}$ by choosing increasing families of sets $A_{j, \varepsilon}^{t}$ describing the damage in the $j$-th material with $\left|A_{j, \varepsilon}^{t}\right|=\frac{1}{2} \lambda_{j \text {,min }}(t)$, setting $A_{\varepsilon}^{t}=A_{1, \varepsilon}^{t} \cup A_{2, \varepsilon}^{t}$ and $u_{\varepsilon}^{t}$ the corresponding solution of $m^{\varepsilon}\left(A^{t}, t\right)$. However the sets $A_{\varepsilon}^{t}$ do not converge to sets as $\varepsilon \rightarrow 0$ except for the trivial cases. In particular this applies for $\frac{p_{2}\left(\beta_{1}+\alpha_{2}\right)}{2 \beta_{1} \alpha_{2}} \leq t \leq \frac{p_{1}\left(\beta_{1}+\alpha_{2}\right)}{2 \beta_{1} \alpha_{2}}$, in which case $\lambda_{2, \min }(t)=1$ and $\lambda_{1, \min }(t)=0$ so that $A_{\varepsilon}^{t}=\varepsilon\left(\mathbb{Z}+\left[\frac{1}{2}, 1\right]\right)$.

\section{A double-damage-set formulation}

The observation above highlights that a weaker notion of convergence of sets must be given in order to describe the behavior of (some solutions of) the sequence of damage problem. One way is to choose particular sequences of damaged sets $A_{j, \varepsilon}^{t}$, for examples intersections of intervals with the $j$-th material. For simplicity we consider intervals $\left[0, \lambda_{j, \varepsilon}(t)\right]$ with one endpoint in 0 , so that

$$
A_{1, \varepsilon}^{t}=\left[0, \lambda_{1, \varepsilon}(t)\right] \cap \varepsilon\left(\mathbb{Z}+\left[0, \frac{1}{2}\right]\right), \quad A_{2, \varepsilon}^{t}=\left[0, \lambda_{2, \varepsilon}(t)\right] \cap \varepsilon\left(\mathbb{Z}+\left[\frac{1}{2}, 1\right]\right) .
$$

Note that under hypothesis (2.16) we have $\lambda_{2, \varepsilon}(t) \geq \lambda_{1, \varepsilon}(t)$ for all $t$. We haven therefore to examine problems (2.13) rewritten in the form

$$
\begin{gathered}
m^{\varepsilon}\left(\lambda_{1, \varepsilon}, \lambda_{2, \varepsilon}, t\right)=\min \left\{\int_{0}^{\lambda_{1, \varepsilon}} \alpha\left(\frac{x}{\varepsilon}\right)\left|u^{\prime}\right|^{2} d x+\int_{\lambda_{1, \varepsilon}}^{\lambda_{2, \varepsilon}} a\left(\frac{x}{\varepsilon}\right)\left|u^{\prime}\right|^{2} d x+\int_{\lambda_{2, \varepsilon}}^{1} \beta\left(\frac{x}{\varepsilon}\right)\left|u^{\prime}\right|^{2} d x:\right. \\
u(0)=0, u(1)=t\}
\end{gathered}
$$

where $a$ is the 1-periodic function with

$$
a(y)=\left\{\begin{array}{ll}
\beta_{1} & \text { for } 0 \leq y<\frac{1}{2} \\
\alpha_{2} & \text { for } \frac{1}{2} \leq y<1
\end{array} .\right.
$$


If $\lambda_{j, \varepsilon} \rightarrow \lambda_{j}$ then these problems converge to

$m_{\mathrm{hom}}\left(\lambda_{1}, \lambda_{2}, t\right)=\min \left\{\underline{\alpha} \int_{0}^{\lambda_{1}}\left|u^{\prime}\right|^{2} d x+\underline{a} \int_{\lambda_{1}}^{\lambda_{2}}\left|u^{\prime}\right|^{2} d x+\underline{\beta} \int_{\lambda_{2}}^{1}\left|u^{\prime}\right|^{2} d x: u(0)=0, u(1)=t\right\}$.

Taking into account that in this case $\int_{A} \gamma(x / \varepsilon) d x \rightarrow \frac{1}{2} \gamma_{2} \lambda_{2}+\frac{1}{2} \gamma_{1} \lambda_{1}$, the limit of $m^{\varepsilon}(t)$ can be written as

$$
\begin{gathered}
m_{\mathrm{hom}}(t)=\min \left\{\int_{0}^{1}\left(\chi_{\left[0, \lambda_{1}\right]}\left(\underline{\alpha}\left|u^{\prime}\right|^{2}+\frac{\gamma_{1}+\gamma_{2}}{2}\right)+\chi_{\left[\lambda_{1}, \lambda_{2}\right]}\left(\underline{a}\left|u^{\prime}\right|^{2}+\frac{\gamma_{2}}{2}\right)+\chi_{\left[\lambda_{2}, 1\right]} \underline{\beta}\left|u^{\prime}\right|^{2}\right) d x\right. \\
\left.: u(0)=0, u(1)=t, 0 \leq \lambda_{1} \leq \lambda_{2} \leq 1\right\}
\end{gathered}
$$

Minimizing first in $\lambda_{1}$ and $\lambda_{2}$ we obtain

$m_{\mathrm{hom}}(t)=\min \left\{\int_{0}^{1} \min \left\{\underline{\alpha}\left|u^{\prime}\right|^{2}+\frac{\gamma_{1}+\gamma_{2}}{2}, \underline{a}\left|u^{\prime}\right|^{2}+\frac{\gamma_{2}}{2}, \underline{\beta}\left|u^{\prime}\right|^{2}\right\} d x: u(0)=0, u(1)=t\right\}$.

This observation highlights that the function $m_{\text {hom }}(t)$ can be expressed as the convex envelope of

$$
\min \left\{\underline{\beta} z^{2}, \frac{2 \alpha_{2} \beta_{1}}{\beta_{1}+\alpha_{2} \beta_{2}} z^{2}+\frac{1}{2} \gamma_{2}, \underline{\alpha}+\frac{1}{2}\left(\gamma_{1}+\gamma_{2}\right)\right\}
$$

which are the three total energy densities corresponding to the mixtures of undamaged material, equally damaged and undamaged material (in the optimal way determined by condition (2.16)), and completely damaged material.

The limit damage motion in this case is given in terms of the two sets $A_{j}^{t}=\left[0, \lambda_{j}(t)\right]$, where $\lambda_{j}(t)$ are the minimizers of problem (2.20), and of the corresponding $u^{t}$. Note that this is possible thanks to the a particular choice of the damage sets $A_{j, \varepsilon}^{t}$, and does not give a description of the behavior of an arbitrary family of solutions $A_{\varepsilon}^{t}, u_{\varepsilon}^{t}$.

\section{Double-threshold formulation}

Also in this case we note that the damage process takes place when $\sigma_{t}$ reaches some particular values. In this case the thresholds are two given by $p_{1}$ and $p_{2}$ (see Fig. 2.3 as compared with Fig. 2.2).

\subsubsection{Homogenization of damage: dissipations leading to a commutabil- ity result}

We now slightly modify the dissipation in the example of the previous section. This will produce a 'commutatibility' result in the quasi static motion outlined above. The first such modification is obtained by imposing that the domain of the dissipation be the set of intervals; i.e.,

$$
D_{\varepsilon}(A)=+\infty \text { if } A \text { is not an interval, }
$$


while $D_{\varepsilon}$ remains unchanged otherwise. In this case in the process described above, at fixed $\varepsilon$ me may remark that the minimal $A_{\varepsilon}^{t}$ will converge to some interval $A^{t}$ for which we may pass to the limit obtaining the problem

$$
\min \left\{F_{\mathrm{hom}, A^{t}}(u)+\bar{\gamma}\left|A^{t}\right|: u(0)=0, u(1)=t\right\}
$$

where

$$
\bar{\gamma}=\frac{\gamma_{1}+\gamma_{2}}{2}
$$

since

$$
\lim _{\varepsilon \rightarrow 0} D_{\varepsilon}\left(A_{\varepsilon}^{t}\right)=\bar{\gamma}\left|A^{t}\right| .
$$

Note that in the previous example this passage was not possible since the $A_{\varepsilon}$ thus defined do not converge to a limit set.

We may conclude then that $A^{t}$ minimizes the corresponding

$$
\begin{aligned}
m_{\text {hom }}(t) & :=\min \left\{F_{\text {hom }, A}(u)+\bar{\gamma}|A|: u(0)=0, u(1)=t, A \text { subinterval of }(0,1)\right\} \\
& =\min \left\{F_{\text {hom }, A}(u)+\bar{\gamma}|A|: u(0)=0, u(1)=t, A \subset(0,1)\right\} \\
& =f_{\text {hom }}^{* *}(t),
\end{aligned}
$$

where

$$
f_{\text {hom }}(z)=\min \left\{\underline{\alpha} z^{2}+\bar{\gamma}, \underline{\beta} z^{2}\right\},
$$

which describes the damage process corresponding to the limit homogenized functionals. Note that in the limit problem we may remove the constraint that $A$ be an interval, since we have already remarked that solutions satisfying such a constraint exist.

\section{Brutal damage}

We consider another dissipation, with

$$
D_{\varepsilon}(A)=\int_{A} \gamma\left(\frac{x}{\varepsilon}\right) d x+\sigma \#(\partial A \cap[0,1])
$$

so that it is finite only on finite unions of intervals.

We may compute the limit of $m^{\varepsilon}(t)$ as above, noticing that for a finite union of intervals, we may pass to the limit (taking possibly into account that the number of intervals may decrease in the limit process), and conclude that the limit damage process corresponds to the functionals $F_{\text {hom, } A}$ and the homogenized dissipation

$$
D_{\text {hom }}(A)=\bar{\gamma}|A|+\sigma \#(\partial A \cap[0,1]) .
$$




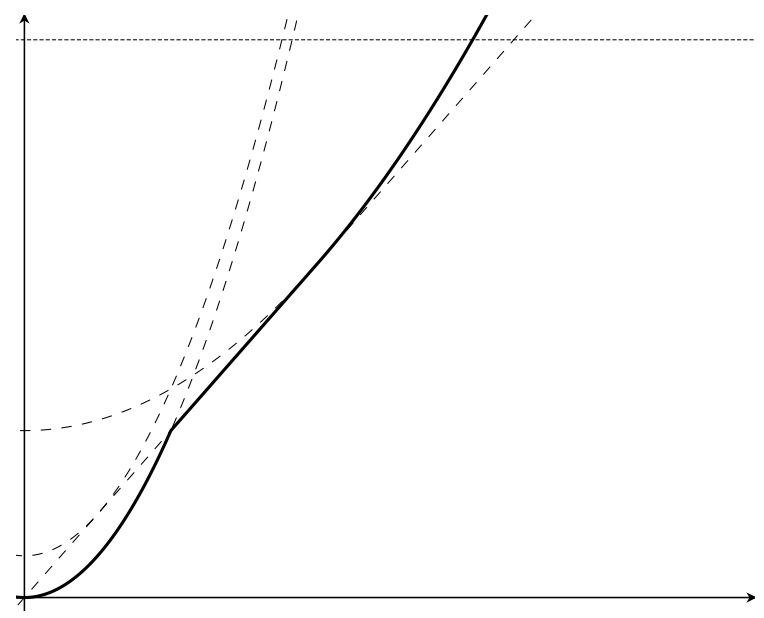

Figure 2.4: minimal energy $m_{\text {hom }}$

Correspondingly, we can compute the minima

$m_{\mathrm{hom}}(t)=\min \left\{F_{\mathrm{hom}, A}(u)+D_{\mathrm{hom}}(A): u(0)=0, u(1)=t, A\right.$ union of subintervals of $\left.(0,1)\right\}$, as

$$
m_{\mathrm{hom}}(t)=\min \left\{m_{\mathrm{hom}}^{0}(t), m_{\mathrm{hom}}^{1}(t)\right\}
$$

where $m_{\text {hom }}^{0}$ corresponds to no damage,

$$
\left.m_{\text {hom }}^{0}(t)=\min \left\{F_{\text {hom }, \emptyset}(u): u(0)=0, u(1)=t\right)\right\}=\underline{\beta} t^{2},
$$

and $m_{\text {hom }}^{1}$ corresponds to $A$ a single interval (not being energetically convenient to have more than one interval),

$$
\begin{aligned}
m_{\mathrm{hom}}^{1}(t) & =\inf \left\{F_{\mathrm{hom}, A}(u)+D_{\mathrm{hom}}(A): u(0)=0, u(1)=t, A \text { subinterval of }(0,1), A \neq \emptyset\right\} \\
& =\min \left\{F_{\mathrm{hom}, A}(u)+\bar{\gamma}|A|: u(0)=0, u(1)=t, A \text { subinterval of }(0,1)\right\}+2 \sigma \\
& =f_{\mathrm{hom}}^{* *}(t)+2 \sigma
\end{aligned}
$$

with $f_{\text {hom }}$ as in (2.23).

The plot of $m_{\text {hom }}$ is reproduced in Fig. 2.4. Note that we follow the undamaged curve until we reach the graph of $m_{\text {hom }}^{1}$, which corresponds to a positive value of the damage area; i.e., the damage is 'brutal' (once it is convenient to damage, we damage a large region). Correspondingly, in Fig. 2.5 we plot the value of $m_{\mathrm{hom}}^{\prime}$ and the derivative of the homogenized energy $\mathcal{E}$ along a cycle 


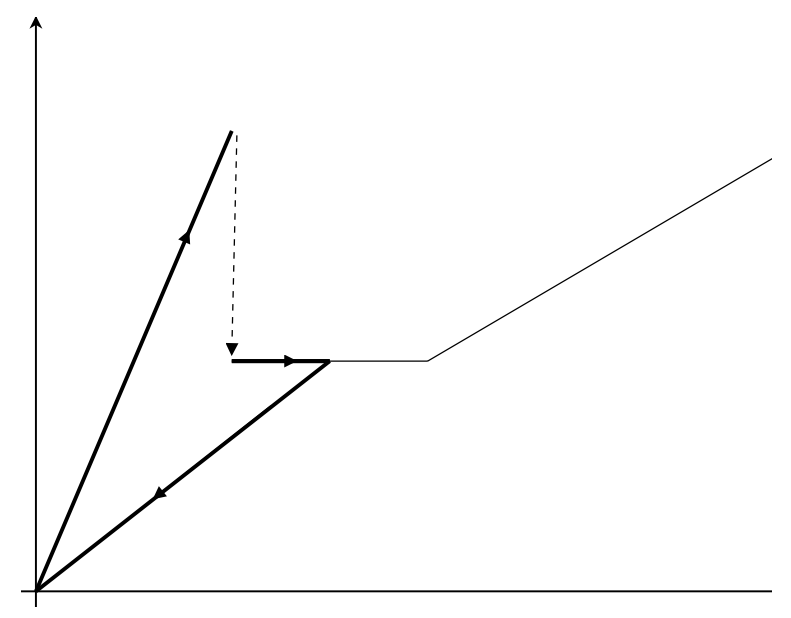

Figure 2.5: plot of $m_{\text {hom }}^{\prime}(t)$ and derivative of the energy along a cycle

\subsubsection{Conditions for commutability}

Motivated by the examples above, we may derive a criterion of commutability of $\Gamma$ convergence and quasi-static motion, which we state in this particular case but is immediately generalized to more abstract situations. This easily follows from the remark that in order to pass to the limit we have to have the convergence of the minimum problems

$$
\min \left\{F_{\varepsilon, A}(u)+D_{\varepsilon}(A): u(0)=0, u(1)=g(t), B_{\varepsilon} \subset A\right\}
$$

with $B_{\varepsilon}$ Borel sets converging to $B$ (in $(2.1) B_{\varepsilon}=\bigcup\left\{A_{s}: s<t\right\}$ ) to

$$
\min \left\{F_{\text {hom }, A}(u)+D_{\text {hom }}(A): u(0)=0, u(1)=g(t), B \subset A\right\} .
$$

Proposition 2.1.3 (commutativity criterion) Let $B_{\varepsilon} \rightarrow B$ and let

$$
\begin{aligned}
G_{\varepsilon}(u, A) & = \begin{cases}F_{\varepsilon, A}(u)+D_{\varepsilon}(A) & \text { if } B_{\varepsilon} \subset A \\
+\infty & \text { otherwise }\end{cases} \\
G_{\mathrm{hom}}(u, A) & = \begin{cases}F_{\mathrm{hom}, A}(u)+D_{\mathrm{hom}}(A) & \text { if } B \subset A \\
+\infty & \text { otherwise. }\end{cases}
\end{aligned}
$$

Suppose that $G_{\varepsilon} \Gamma$-converges to $G_{\text {hom }}$ with respect to the converge $L^{2} \times L^{1}$-convergence (the latter is understood as the convergence of the characteristic functions of sets). Then if a sequence of solutions $\left(u_{\varepsilon}^{t}, A_{\varepsilon}^{t}\right)$ to the evolutions related to the energies $F_{\varepsilon, A}$, dissipation $D_{\varepsilon}$ and boundary conditions given by $g$ is such that (up to subsequences) for all $t u_{\varepsilon}^{t}$ converges to some $u^{t}$ in $L^{2}$ and $B_{\varepsilon}^{t}=\bigcup\left\{A_{\varepsilon}^{s}: s<t\right\}$ converges to some $B^{t}$ in $L^{1}$, then it converges (up to subsequences) to a solution to the evolution related to the energies $F_{\text {hom, }}$, dissipation $D_{\text {hom }}$ and boundary conditions given by $g$. 
This criterion follows from the fundamental theorem of $\Gamma$-convergence, upon noting that the boundary conditions are compatible with the convergence of minima regardless to the constraint $B_{\varepsilon} \subset A$.

Remark 2.1.4 We may apply Proposition 2.1.3 to the two examples in Section 2.1.3. In fact, in both cases the boundedness of the dissipation implies that $A_{\varepsilon}^{t}$ and hence $B_{\varepsilon}^{t}$ are (increasing with $t$ ) intervals (or finite union of intervals in the second case), so that the pre compactness of $B_{\varepsilon}^{t}$ is guaranteed. The convergence for all $t$ follows from an application of Helly's theorem.

We cannot apply Proposition 2.1.3 to the solutions in Section 2.1.2. Indeed, except for the trivial cases when $A_{\varepsilon}^{t}=\emptyset$ or $A_{\varepsilon}^{t}=(0,1)$, these do not converge strongly in $L^{1}$ but only weakly.

\subsubsection{Relaxed evolution}

The criterion above suggests, in case it is not satisfied, to examine the behavior of the functionals (2.27) with respect to the $L^{2} \times L^{1}$-weak convergence. In this case the limit of a sequence of characteristic functions may not be a characteristic function itself, so that the domain of the $\Gamma$-limit will be the space of pairs $(u, \theta)$, with $0 \leq \theta \leq 1$. This formulation will necessarily be more complex, but will capture the behavior of all sequences $A_{\varepsilon}^{t}, u_{\varepsilon}^{t}$.

Proposition 2.1.5 (relaxed total energies) If hypothesis (2.16) holds, then the $\Gamma$-limit of the functionals (2.27) with respect to the $L^{2} \times L^{1}$-weak convergence is given by the functional (r stands for 'relaxed')

$$
G_{\mathrm{hom}}^{r}(u, \theta)=\int_{(0,1)} f_{\mathrm{hom}}\left(\theta, u^{\prime}\right) d x+\int_{(0,1)} \gamma_{\mathrm{hom}}(\theta) d x
$$

where

$$
f_{\text {hom }}(\theta, z)= \begin{cases}\frac{2 \alpha_{2} \beta_{1} \beta_{2}}{2 \theta \beta_{1} \beta_{2}+(1-2 \theta) \alpha_{2} \beta_{1}+\alpha_{2} \beta_{2}} z^{2} & \text { if } 0 \leq \theta \leq \frac{1}{2} \\ \frac{2 \alpha_{1} \alpha_{2} \beta_{1}}{2(1-\theta) \alpha_{1} \alpha_{2}+(2 \theta-1) \alpha_{2} \beta_{1}+\alpha_{1} \beta_{1}} z^{2} & \text { if } \frac{1}{2} \leq \theta \leq 1\end{cases}
$$

and the dissipation energy density is

$$
\gamma_{\mathrm{hom}}(\theta)= \begin{cases}\gamma_{2} \theta & \text { if } 0 \leq \theta \leq \frac{1}{2} \\ \frac{1}{2} \gamma_{2}+\gamma_{1}\left(\theta-\frac{1}{2}\right) & \text { if } \frac{1}{2} \leq \theta \leq 1\end{cases}
$$


Proof. We do not dwell on this proof, since it is variation of the usual homogenization theorem. A lower bound is obtained by minimizing on each periodicity cell. Upon scaling we are led to computing

$$
\begin{gathered}
\phi(z, \theta):=\min \left\{\int_{A} \alpha(y)\left|u^{\prime}\right|^{2} d y+\int_{(0,1) \backslash A} \beta(y)\left|u^{\prime}\right|^{2} d y+\int_{A} \gamma(y) d y\right. \\
:|A|=\theta, u(0)=0, u(1)=z\} .
\end{gathered}
$$

By a direct computation we get

$$
\phi(z, \theta)=f_{\text {hom }}(\theta, z)+\gamma_{\text {hom }}(\theta) .
$$

Since $\phi$ is convex in the pair $(z, \theta)$, its integral is lower semicontinuous in $L^{2} \times L^{1}$-weak, and hence is a candidate for the $\Gamma$-liminf. The proof of the limsup inequality is obtained by density, first dealing with $u$ piecewise affine and $\theta$ piecewise constant.

Remark 2.1.6 The limit of problems (2.25) with $B_{\varepsilon}$ converging weakly to some $\phi$ will be of the form

$$
\min \left\{G_{\text {hom }}^{r}(u, \theta): u(0)=0, u(1)=g(t), \phi \leq \theta\right\} .
$$

As above, we only consider the case $g(t)=t$, and the problem

$$
m^{r}(t)=\min \left\{G_{\text {hom }}^{r}(u, \theta): u(0)=0, u(1)=g(t)\right\} .
$$

We have

$$
m^{r}(t)=\min \left\{\int_{0}^{1} \min _{0 \leq \theta \leq 1}\left\{f_{\text {hom }}\left(\theta, u^{\prime}\right)+\gamma_{\text {hom }}(\theta)\right\} d x: u(0)=0, u(1)=g(t)\right\} .
$$

A direct computation shows that

$$
\min _{0 \leq \theta \leq 1}\left\{f_{\text {hom }}(\theta, z)+\gamma_{\text {hom }}(\theta)\right\}=m(z)
$$

with $m$ the one in Section 2.1.2; hence, by convexity $m^{r}(z)=m(z)$. Moreover, again using the convexity of $m$, a solution is simply given by $u_{t}(x)=t x$ and correspondingly $\theta=\theta(t)$ constant equal to the minimizer of (2.35) with $z=t$; namely

$$
\theta(t)= \begin{cases}0 & \text { if }|t| \leq \frac{p_{2}}{\underline{\beta}} \\ \frac{p_{2}^{2}}{\gamma_{2}}\left(\frac{t}{p_{2}}-\frac{1}{\underline{\beta}}\right) & \text { if } \frac{p_{2}}{\underline{\beta}}<|t|<\frac{p_{2}\left(\beta_{1}+\alpha_{2}\right)}{2 \beta_{1} \alpha_{2}} \\ \frac{1}{2} & \text { if } \frac{p_{2}\left(\beta_{1}+\alpha_{2}\right)}{2 \beta_{1} \alpha_{2}} \leq|t| \leq \frac{p_{1}\left(\beta_{1}+\alpha_{2}\right)}{2 \beta_{1} \alpha_{2}} \\ 1+\frac{p_{1}^{2}}{\gamma_{1}}\left(\frac{t}{p_{1}}-\frac{1}{\underline{\alpha}}\right) & \text { if } \frac{p_{1}\left(\beta_{1}+\alpha_{2}\right)}{2 \beta_{1} \alpha_{2}}<|t|<\frac{p_{1}}{\underline{\alpha}} \\ 1 & \text { if }|t| \geq \frac{p_{1}}{\underline{\alpha}} .\end{cases}
$$




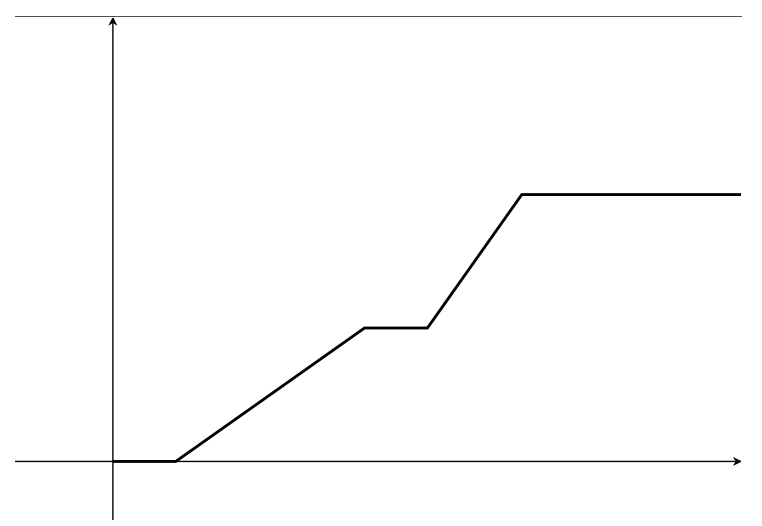

Figure 2.6: value of the damage $\theta(t)$

Note that $\theta(t)=\frac{\lambda_{1, \min }(t)+\lambda_{2, \min }(t)}{2}$ with $\lambda_{j, \min }$ given by (2.17) and (2.18). The solution with $\theta$ constant corresponds to equi-distributed damage. Note that we have infinitely many solutions, among which the ones described above in terms of $A_{1}^{t}$ and $A_{2}^{t}$.

\subsection{Mielkian theory of rate-independent evolution}

The examples in the previous theory can be framed in a general theory of rate-independent variational evolution. We introduce some of the concepts of the theory that are relevant to our presentation, without being precise in the hypotheses on spaces and topologies

Definition 2.2.1 Let $\mathcal{F}=\mathcal{F}(t, \cdot)$ be a time-parameterized energy functional and $\mathcal{D}$ be a dissipation functional, which we assume to be positively-homogeneous of degree one; i.e. $\mathcal{D}(s U)=s \mathcal{D}(U)$ if $s>0$. Then $U$ is an energetic solution for the evolution inclusion

$$
\partial \mathcal{D}(\dot{U})+\partial_{U} \mathcal{F}(t, U) \ni 0
$$

if the following two conditions hold:

(S) global stability for all $t$ and $\widehat{U}$ we have

$$
\mathcal{F}(t, U(t)) \leq \mathcal{F}(t, \widehat{U})+\mathcal{D}(\widehat{U}-U(t))
$$

(E) energy inequality for all $t$

$$
\mathcal{F}(t, U(t))+\int_{0}^{t} D(\dot{U}) \leq \mathcal{F}(0, U(0))+\int_{0}^{t} \partial_{s} \mathcal{F}(s, U(s)) d s .
$$


In this formula the integral $\int_{0}^{t} D(\dot{U})$ must be understood in the sense of measures, and can be equivalently defined as

$$
\sup \left\{\sum_{i=1}^{n} D\left(U\left(t_{i}\right)-U\left(t_{i-1}\right)\right): 0=t_{0}<t_{1}<\cdots<t_{n}=t\right\} .
$$

If $U$ is an absolutely continuous function then the integral reduces to $\int_{0}^{t} D(\dot{U}(s)) d s$.

Remark 2.2.2 Under mild assumptions from (S) it can be deduced that in (E) the equality sign holds, so that we have an energy conservation identity. This identity states that the difference of the energy at a final and an initial state equals the difference of the work of the applied actions and the total dissipation along the path.

Remark 2.2.3 In the case of damage we have $U=(u, v)$,

$$
\mathcal{F}(t, u, v)= \begin{cases}\int_{0}^{1}\left(\alpha v\left|u^{\prime}\right|^{2}+\beta(1-v)\left|u^{\prime}\right|^{2}\right) d x & \text { if } v \in\{0,1\} \text { a.e., } u(0)=0, u(1)=g(t) \\ +\infty & \text { otherwise, }\end{cases}
$$

and

$$
\mathcal{D}(U)= \begin{cases}\gamma \int_{0}^{1} v d x & \text { if } v \in\{0,1\} \text { a.e. } \\ +\infty & \text { otherwise. }\end{cases}
$$

Condition (S) is meaningful only if $\widehat{U}=(u, v)$ and $U(t)=\left(u^{t}, v^{t}\right)$ satisfy $v=\chi_{A}$ and $v^{t}=\chi_{A^{t}}$ with $A^{t} \subset A$, so that $(\mathrm{S})$ implies that $u^{t}$ and $A^{t}$ are minimizers for (2.1). Conversely, it can be checked that the solutions to the damage evolution satisfy the energy inequality as an identity.

Remark 2.2.4 (rate-independence) The requirement that $\mathcal{D}$ be positively homogeneous of degree one implies that the solution is rate-independent; i.e., that if we consider a re-parameterization of the energy $\widetilde{\mathcal{F}}(t, U)=\mathcal{F}(\varphi(t), U)$ via an increasing diffeomorphism $\varphi$, then the energetic solutions $\widetilde{U}$ of the corresponding evolution inclusion are exactly the $\widetilde{U}(t)=U(\varphi(t))$ with $U$ energetic solutions of the corresponding evolution inclusion for $\mathcal{F}$.

Example 2.2.5 (mechanical play/hysteresis) The prototypical example of an evolution inclusion is by taking $U=x \in \mathbb{R}$ and

$$
\mathcal{F}(t, x)=\frac{x^{2}}{2}-t x, \quad \mathcal{D}(x)=|x| .
$$


In this case we can write explicitly $\partial|\dot{x}|+x-t \ni 0$ as

$$
\begin{cases}\dot{x}>0 & \text { if } x=t-1 \\ \dot{x}<0 & \text { if } x=t+1 \\ \dot{x}=0 & \text { if } t-1 \leq x \leq t+1\end{cases}
$$

The solution with $x(0)=x_{0} \in[-1,1]$ is

$$
x(t)= \begin{cases}0 & \text { if } t \leq 1+x_{0} \\ t-1 & \text { if } t>1+x_{0}\end{cases}
$$

If we take a non-monotone load $g(t)=T-|t-T|$ with $T>1+x_{0}$ and

$$
\mathcal{F}(t, x)=\frac{x^{2}}{2}-g(t) x, \quad \mathcal{D}(x)=|x| .
$$

then the solution $x$ is as above for $t \leq T$, and given solving $\partial|\dot{x}|+x-(2 T-t) \ni 0$ by

$$
x(t)= \begin{cases}T-1 & \text { if } T \leq t \leq T+2 \\ 2 T-t+1=g(t)+1 & \text { if } t \geq T+2\end{cases}
$$

This solution shows a hysteretic behavior of this system, whose trajectory in the $g$ - $x$ plane is represented in Fig. 2.7

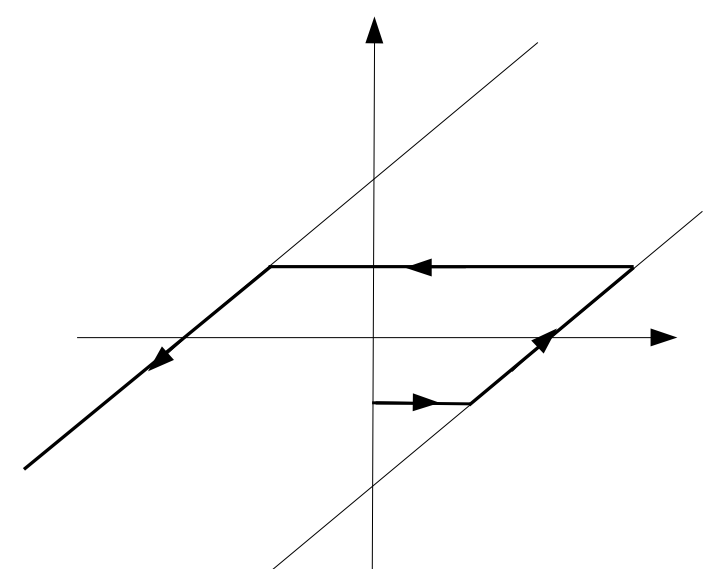

Figure 2.7: hystheretic trajectory

Remark 2.2.6 (solutions obtained by time-discretization) Some energetic solutions can be obtained as limits of discrete schemes as follows: fix $\tau>0$ and define $U_{k}^{\tau}$ recursively by setting $U_{0}^{\tau}=U_{0}$, and choosing $U_{k}^{\tau}$ as a solution of the minimum problem

$$
\min _{\widehat{U}}\left\{\mathcal{F}(\tau k, \widehat{U})+\mathcal{D}\left(\widehat{U}-U_{k-1}^{\tau}\right)\right\}
$$


Define the continuous trajectory $U^{\tau}(t)=U_{\lfloor t / \tau\rfloor}^{\tau}$. Under suitable assumptions the limits of (subsequences of) $U^{\tau}$ are energetic solution of the variational inclusion for $\mathcal{F}$ and $\mathcal{D}$.

\subsubsection{Stability}

We can give a stability result with respect to $\Gamma$-convergence. As remarked in the case of damage the separate $\Gamma$-convergence of $\mathcal{F}_{\varepsilon}$ and $\mathcal{D}_{\varepsilon}$ may not be sufficient to describe the limit of the corresponding variational motions.

Theorem 2.2.7 Suppose that $\mathcal{F}$ and $\mathcal{D}$ are lower bounds for $\mathcal{F}_{\varepsilon}$ and $\mathcal{D}_{\varepsilon}$, that $U_{\varepsilon}$ are energetic solutions converging pointwise to some $U$ as $\varepsilon \rightarrow 0$, that the initial data are well-prepared; i.e., that

$$
\lim _{\varepsilon \rightarrow 0} \mathcal{F}_{\varepsilon}\left(0, U_{\varepsilon}(0)\right)=\mathcal{F}_{\varepsilon}(0, U(0)),
$$

that we have convergence of the external actions

$$
\lim _{\varepsilon \rightarrow 0} \int_{0}^{t} \partial_{s} \mathcal{F}_{\varepsilon}\left(s, U_{\varepsilon}(s)\right) d s=\int_{0}^{t} \partial_{s} \mathcal{F}(s, U(s)) d s \quad \text { for all } t,
$$

and that the following mutual recovery sequence existence condition holds: for all $t$ and all $\widehat{U}$ there exists a sequence $\widehat{U}_{\varepsilon}$ such that

$$
\begin{gathered}
\limsup _{\varepsilon \rightarrow 0}\left(\mathcal{F}_{\varepsilon}\left(t, \widehat{U}_{\varepsilon}\right)-\mathcal{F}_{\varepsilon}\left(t, U_{\varepsilon}(t)\right)+\mathcal{D}_{\varepsilon}\left(\widehat{U}_{\varepsilon}-U_{\varepsilon}(t)\right)\right) \\
\leq \mathcal{F}(t, \widehat{U})-\mathcal{F}(t, U(t))+\mathcal{D}(\widehat{U}-U(t))
\end{gathered}
$$

Then $U$ is an energetic solution for the limit energy and dissipation.

Proof. Let $0=t_{0}<t_{1}<\cdots<t_{n}=t$; by the liminf inequality for $D_{\varepsilon}$ and (2.36) we then have

$$
\sum_{i=1}^{n} \mathcal{D}\left(U\left(t_{i}\right)-U\left(t_{i-1}\right)\right) \leq \liminf _{\varepsilon \rightarrow 0} \sum_{i=1}^{n} \mathcal{D}_{\varepsilon}\left(U_{\varepsilon}\left(t_{i}\right)-U_{\varepsilon}\left(t_{i-1}\right)\right) \leq \liminf _{\varepsilon \rightarrow 0} \int_{0}^{t} \mathcal{D}_{\varepsilon}\left(\dot{U}_{\varepsilon}\right) .
$$

Taking into account the liminf inequality for $\mathcal{F}_{\varepsilon}$ and the convergence hypotheses on initial data and external actions we then obtain

$$
\begin{aligned}
\mathcal{F}(t, U(t))+\int_{0}^{t} D(\dot{U}(s)) d s & \leq \liminf _{\varepsilon \rightarrow 0}\left(\mathcal{F}_{\varepsilon}\left(0, U_{\varepsilon}(0)\right)+\int_{0}^{t} \partial_{s} \mathcal{F}_{\varepsilon}\left(s, U_{\varepsilon}(s)\right) d s\right) \\
& =\mathcal{F}(0, U(0))+\int_{0}^{t} \partial_{s} \mathcal{F}(s, U(s)) d s
\end{aligned}
$$

so that (E) holds. 
Take any test $\widehat{U}$ and use the mutual recovery sequence $\widehat{U}_{\varepsilon}$ to obtain

$$
\mathcal{F}(t, \widehat{U})-\mathcal{F}(t, U(t))+\mathcal{D}(\widehat{U}-U(t)) \geq 0
$$

i.e. the inequality in $(\mathrm{S})$, from the same inequality for $U_{\varepsilon}$.

Proposition 2.2.8 (necessary and sufficient conditions) (i) Let $\mathcal{F}_{\varepsilon} \Gamma$-converge to $\mathcal{F}$ and $\mathcal{D}_{\varepsilon}$ converge continuously to $\mathcal{D}$. Then the mutual recovery sequence condition is satisfied;

(ii) Assume that $\mathcal{F}_{\varepsilon}$ and $\mathcal{D}_{\varepsilon} \Gamma$-converge to $\mathcal{F}$ and $\mathcal{D}$, that $U_{\varepsilon}(t)$ is a recovery sequence for $F_{\varepsilon}$ at $U(t)$ and that the mutual recovery sequence condition holds with $\widehat{U}_{\varepsilon} \rightarrow \widehat{U}$. Then $\mathcal{G}_{\varepsilon}(V)=\mathcal{F}_{\varepsilon}(t, V)+\mathcal{D}_{\varepsilon}\left(V-U_{\varepsilon}(t)\right) \Gamma$-converges to $\mathcal{G}(V)=\mathcal{F}(t, U(t))+\mathcal{D}(V-U(t))$.

Proof. (i) follows by taking $\widehat{U}_{\varepsilon}$ any recovery sequence for $\mathcal{F}_{\varepsilon}(t, \widehat{U})$.

(ii) is an immediate consequence of the fact that $\mathcal{F}+\mathcal{D}$ is a lower bound for $\mathcal{F}_{\varepsilon}+\mathcal{D}_{\varepsilon}$, while the mutual recovery sequence provides a recovery sequence for $\mathcal{F}(t, U(t))+\mathcal{D}(V-U(t))$.

Example 2.2.9 (an example with relaxed evolution) In $\mathbb{R}^{2}$ with $U=(x, y)$, consider the initial datum $u_{\varepsilon}(0)=(0,0)$ and the energy and dissipation

$$
\mathcal{F}_{\varepsilon}(t, U)=\frac{1}{2} x^{2}+\frac{1}{2 \varepsilon^{2}}(y-\varepsilon x)^{2}-t x, \quad \mathcal{D}_{\varepsilon}(U)=|x|+\frac{1}{\varepsilon}|y|
$$

with $\Gamma$-limits

$$
\mathcal{F}(t, U)=\frac{1}{2} x^{2}-t x, \quad \mathcal{D}(U)=|x|
$$

with domain $\{y=0\}$.

The solution to the differential inclusion for $\mathcal{F}$ and $\mathcal{D}$ with initial datum $(0,0)$ is given by $x(t)$ as in $(2.37)$ with $x_{0}=0$, and $y(t)=0$. On the other hand, the solutions to the differential inclusions $U_{\varepsilon}$ can be computed explicitly, and they tend to $U=(x, y)$ defined by $y(t)=0$ and

$$
x(t)= \begin{cases}0 & \text { if } t \leq 1 \\ \frac{t-1}{2} & \text { if } 1 \leq t \leq 3 \\ t-2 & \text { if } t \geq 3 .\end{cases}
$$

In this case we do not have convergence of the solutions. However, we can compute the $\Gamma$-limit of the $\operatorname{sum} \mathcal{F}_{\varepsilon}+\mathcal{D}_{\varepsilon}$, whose domain is $\{y=0\}$. Recovery sequences for $(x, 0)$ can be looked for of the form $(x, \varepsilon z)$. By minimizing in $z$ we easily get that this $\Gamma$-limit is

$$
\mathcal{G}(x)=\frac{1}{2} x^{2}-t x+|x|+\psi(x),
$$


where

$$
\psi(x)=\min \left\{\frac{1}{2}(z-x)^{2}+|z|\right\}=\min \left\{\frac{x^{2}}{2}, \frac{1}{2}+|| x|-1|\right\}
$$

whose derivative is

$$
\psi^{\prime}(x)=(x \wedge 1) \vee(-1) .
$$

It is easily seen that the function $x(t)$ above is the solution of

$$
\partial|\dot{x}|+\mathcal{F}_{0}^{\prime}(x)=\partial|\dot{x}|+x-t+\psi^{\prime}(x) \ni 0,
$$

where $\mathcal{F}_{0}(x)=\mathcal{G}(x)-|x|=\frac{1}{2} x^{2}-t x+\psi(x)$. This energy $\mathcal{F}_{0}$ can then be regarded as the relaxed effective energy describing the limit behavior of the system.

\subsection{Francfort and Marigo's Variational Theory of Fracture}

A very interesting application of the theory outline above is to variational models of Fracture following the formulation given by Griffith in the 1920's. In this case it is maybe clearer the definition via time-discrete motions (see Remark 2.2.6) given as follows.

We consider the antiplane case where the variable $u$ representing the displacement is scalar. $\Omega$ a bounded open subset of $\mathbb{R}^{n}$ will be the reference configuration of a linearly elastic material subject to brittle fracture as a consequence of a varying boundary condition $u=g(t)$ on $\partial \Omega$. $K$ will be a closed set representing the crack location in the reference configuration. We consider the case $g(0)=0$, and set $K_{0}=\emptyset$.

With fixed $\tau>0$ we define $u_{0}^{\tau}=0, K_{0}^{\tau}=K_{0}$ and $u_{k}^{\tau}, K_{k}^{\tau}$ recursively as minimizers of the problem

$$
\begin{gathered}
\min \left\{\int_{\Omega \backslash K}|\nabla u|^{2} d x+\mathcal{H}^{n-1}\left(K \backslash K_{k-1}^{\tau}\right): K_{k-1}^{\tau} \subset K=\bar{K} \subset \bar{\Omega},\right. \\
\left.u \in H^{1}(\Omega \backslash K), u=g(t) \text { on } \partial \Omega \backslash K\right\} .
\end{gathered}
$$

where $\mathcal{H}^{n-1}$ denotes the $(n-1)$-dimensional Hausdorff measure. In this way $K_{k}^{\tau}$ is an increasing sequence of closed sets. Note that part of the crack may also lie on the boundary of $\Omega$, in which case the boundary condition is satisfied only on $\partial \Omega \backslash K$.

In this formulation we have an elastic energy defined by

$$
\mathcal{F}(t, u, K)= \begin{cases}\int_{\Omega \backslash K}|\nabla u|^{2} d x & \text { if } u \in H^{1}(\Omega \backslash K) \text { and } u=g(t) \text { on } \partial \Omega \backslash K \\ +\infty & \text { otherwise, }\end{cases}
$$

and a dissipation

$$
\mathcal{D}(K)= \begin{cases}\mathcal{H}^{n-1}(K) & \text { if } K=\bar{K} \subset \bar{\Omega} \\ +\infty & \text { otherwise }\end{cases}
$$


The existence of minimizing pairs for $(u, K)$ is not at all trivial. One way is by using the theory of $S B V$ functions; i.e., functions of bounded variation $u$ whose distributional derivative is a measure that can be written as a sum of a measure absolutely continuous with respect to the Lebesue measure and a measure absolutely continuous with respect to the restriction of the $(n-1)$-dimensional Hausdorff measure to the complement of the Lebesgue points of $u$, the latter denoted by $S(u)$. For such functions the approximate gradient $\nabla u$ exists at almost all points. We can therefore define for all closed $K$ the energy

$$
\mathcal{E}_{K}(u)=\int_{\Omega \backslash K}|\nabla u|^{2} d x+\mathcal{H}^{n-1}(S(u) \cap(\Omega \backslash K)) .
$$

Such energies are $L^{1}$-lower semicontinuous and coercive, so that existence of weak solutions in $S B V(\Omega)$ are ensured from the direct methods of the Calculus of Variations. Regularity results give that $\mathcal{H}^{d-1}(\overline{S(u)} \backslash S(u))=0$ for minimizing $u$, so that to a minimizing $u \in$ $S B V\left(\Omega \backslash K_{k-1}^{\tau}\right)$ of

$$
\min \left\{\mathcal{E}_{K_{k-1}^{\tau}}(u): u \in S B V\left(\Omega \backslash K_{k-1}^{\tau}\right), u=g(t) \text { on } \partial \Omega \backslash\left(S(u) \cup K_{k-1}^{\tau}\right)\right\}
$$

corresponds a minimizing pair $K_{k}^{\tau}=K_{k-1}^{\tau} \cup \overline{S(u)}$ and $u_{k}^{\tau}=u_{\mid \Omega \backslash K_{k}^{\tau}} \in H^{1}\left(\Omega \backslash K_{k}^{\tau}\right)$ for (2.39).

The passage from a discrete trajectory $u^{\tau}$ to a continuous one $u$ for all $t$ letting $\tau \rightarrow 0$ is possible thanks to some monotonicity arguments. The delicate step is the proof that such $u$ still satisfies the global stability property, which is ensured by a transfer lemma, which allows to approximate test $\widehat{u}$ in the limit stability estimate with a sequence $\widehat{u}_{\tau}$ that can be used in the stability estimate holding for $u^{\tau}(t)$.

Remark 2.3.1 (existence of fractured solutions) Note that for large enough values of the boundary condition $g(t)$ we will always have a solution with $K^{t} \neq \emptyset$. Indeed consider the case $g(t)=t g_{0}$ with $g_{0} \neq 0$ on $\partial \Omega$. If $K^{t}=\emptyset$ then the corresponding $u^{t}$ is a minimizer of

$$
\min \left\{\int_{\Omega}|\nabla u|^{2} d x: u=t g_{0} \text { on } \partial \Omega\right\}=t^{2} \min \left\{\int_{\Omega}|\nabla u|^{2} d x: u=g_{0} \text { on } \partial \Omega\right\}=: t^{2} C_{0} .
$$

On the other hand we can use as test function $u=0$ and as test set $K=\partial \Omega$ in (2.39), for which the total energy is $C_{1}=\mathcal{H}^{d-1}(\partial \Omega)$. This shows that for $t^{2} C_{0}>C_{1}$ we cannot have $K=\emptyset$.

Remark 2.3.2 (the one-dimensional case) In the one-dimensional case the functional $\mathcal{E}$ reduces to the energy $F$ obtained as a limit in Section 1.5.5 with the normalization $2 J^{\prime \prime}(0)=J(\infty)=1$, since $\mathcal{H}^{0}(K)=\#(K)$. Note that in this case the domain of $\mathcal{E}$ reduces to piecewise- $H^{1}$ functions. If $\Omega=(0,1)$ then the time-continuous solutions are of the form

$$
\left(u^{t}(x), K^{t}\right)= \begin{cases}(g(t) x, \emptyset) & \text { for } t \leq t_{c} \\ \left(g(t) \chi_{\left(x_{0}, 1\right)}(x),\left\{x_{0}\right\}\right) & \text { for } t>t_{c}\end{cases}
$$


or

$$
\left(u^{t}(x), K^{t}\right)= \begin{cases}(g(t) x, \emptyset) & \text { for }<t_{c} \\ \left(g(t) \chi_{\left(x_{0}, 1\right)}(x),\left\{x_{0}\right\}\right) & \text { for } t \geq t_{c}\end{cases}
$$

where $x_{0} \in[0,1]$ and $t_{c}$ is any value with $g\left(t_{c}\right)=1$ and $g(s) \leq 1$ for $s<t_{c}$. This nonuniqueness is due to the fact that for $g(t)=1$ we have two possible types of solutions $u(x)=x$ and $u(x)=\chi_{\left(x_{0}, 1\right)}(x)$.

\subsubsection{Homogenization of fracture}

The interpretation of fracture energies as functionals defined in $S B V$ allows to consider the $L^{1}$-convergence in $S B V$ along sequences with equibounded energy (2.40). With respect to such a convergence we can consider stability issues for energies and dissipations related to the oscillating total energy

$$
\mathcal{E}_{\varepsilon}(u)=\int_{\Omega \backslash K} a_{b}\left(\frac{x}{\varepsilon}\right)|\nabla u|^{2} d x+\int_{S(u) \cap(\Omega \backslash K)} a_{f}\left(\frac{x}{\varepsilon}\right) d \mathcal{H}^{n-1}
$$

(here the coefficients $a_{b}$ and $a_{f}, b$ for bulk, $f$ for fracture, are periodic functions). In this case the limit of the total energies $\mathcal{E}_{\varepsilon}$ is the sum of the energies obtained separately as limits of the energy and the dissipation parts (with respect to the same convergence), and has the form

$$
\mathcal{E}_{\mathrm{hom}}(u)=\int_{\Omega \backslash K}\left\langle A_{\mathrm{hom}} \nabla u, \nabla u\right\rangle d x+\int_{S(u) \cap(\Omega \backslash K)} \varphi_{\mathrm{hom}}(\nu) d \mathcal{H}^{n-1},
$$

where $\nu$ denotes the measure-theoretical normal to $S(u)$. Note that the homogenized $A_{\text {hom }}$ is the same given by the homogenization process in $H^{1}$, while $\varphi_{\text {hom }}$ is an effective fracture energies obtained by optimization on oscillating fractures, related to the homogenization of perimeter functionals. Thanks to this remark it is possible to show that the energetic solutions for $\mathcal{E}_{\varepsilon}$ converge to energetic solutions of $\mathcal{E}_{\text {hom }}$. In terms of construction of mutual recovery sequences this is possible since internal energy and dissipation can be optimized separately, contrary to what happens for the damage case, where both terms involve bulk integrals.

\subsection{References to Chapter 2}

Analyses of damage models linked to our presentation are contained in the work by Francfort and Marigo [26]. The higher-dimensional case is studied in a paper by Francfort and Garroni [24]. A threshold-based formulation is introduced by Garroni and Larsen [28]. The 
examples in Section 2.1.3 have been part of the course exam of B. Cassano and D. Sarrocco at Sapienza University in Rome.

An analysis of rate-independent processes is contained in the review article by Mielke [34]. The definitions given here can be traced back to the works by Mielke, Theil and Levitas, [37] and [38]. The stability with respect to $\Gamma$-convergence is analyzed in the paper by Mielke, Roubiček, and Stefanelli [36]. Most of Section 2.2 is taken from a lecture given by Ulisse Stefanelli during the course at the University of Pavia.

An account of the variational theory of fracture (introduced in [27]) is contained in the book by Bourdin, Francfort, and Marigo [8]. The fundamental transfer lemma is contained in the seminal paper by Francfort and Larsen [25]. 


\section{Chapter 3}

\section{Local minimization as a selection criterion}

We will now consider some local minimization issues. By a local minimizer of $F$ we mean a point $u$ such that there exists $\delta>0$ such that

$$
F\left(u_{0}\right) \leq F(u) \quad \text { if } \quad d\left(u, u_{0}\right) \leq \delta .
$$

The $\Gamma$-limit $F$ of a sequence $F_{\varepsilon}$ is often taken as a simplified description of the energies $F_{\varepsilon}$, where unimportant details have been averaged out still keeping the relevant information about minimum problems. As far as global minimization problems are concerned this is ensured by the fundamental theorem of $\Gamma$-convergence, but this is in general false for local minimization problems. Nevertheless, if some information on the local minima is known, we may use the fidelity of the description of local minimizers as a way to 'correct' $\Gamma$-limits. In order to so that, we first introduce some notions of equivalence by $\Gamma$-convergence, and then show how to construct simpler equivalent theories as perturbations of the $\Gamma$-limit $F$ in some relent examples.

\subsection{Equivalence by $\Gamma$-convergence}

Definition 3.1.1 Let $\left(F_{\varepsilon}\right)$ and $\left(G_{\varepsilon}\right)$ be sequences of functionals on a separable metric space $X$. We say that they are equivalent by $\Gamma$-convergence (or $\Gamma$-equivalent) if there exists a sequence $\left(m_{\varepsilon}\right)$ of real numbers such that if $\left(F_{\varepsilon_{j}}-m_{\varepsilon_{j}}\right)$ and $\left(G_{\varepsilon_{j}}-m_{\varepsilon_{j}}\right)$ are $\Gamma$-converging sequences, their $\Gamma$-limits coincide and are proper (i.e., not identically $+\infty$ and not taking the value $-\infty$ ).

Remark 3.1.2 (i) since $\Gamma$-convergence is sequentially compact (i.e., every sequence has a $\Gamma$-converging subsequence), the condition in the definition is never empty. On the set of 
proper lower-semicontinuous functionals the definition above is indeed an equivalence relation (in particular any sequence $\left(F_{\varepsilon}\right)$ is equivalent to itself, regardless to its convergence);

(ii) note that if $F_{\varepsilon} \Gamma$-converge to $F$ and $G_{\varepsilon} \Gamma$-converge to $G$ then equivalence amounts to $F=G$ and $F$ proper, and $\left(F_{\varepsilon}\right)$ is equivalent to the constant sequence $F$;

(iii) the addition of the constants $m_{\varepsilon}$ allows to consider and discriminate among diverging sequences (whose limit is not proper). For example the sequences of constants $F_{\varepsilon}=1 / \varepsilon$ and $G_{\varepsilon}=1 / \varepsilon^{2}$ are not equivalent, even though they diverge to $+\infty$. Note instead that $F_{\varepsilon}(x)=x^{2} / \varepsilon$ and $G_{\varepsilon}(x)=x^{2} / \varepsilon^{2}$ are equivalent.

Definition 3.1.3 (parameterized and uniform equivalence) For all $\lambda \in \Lambda$ let $\left(F_{\varepsilon}^{\lambda}\right)$ and $\left(G_{\varepsilon}^{\lambda}\right)$ be sequences of functionals on a separable metric space $X$. We say that they are equivalent by $\Gamma$-convergence if for all $\lambda$ they are equivalent according to the definition above. If $\Lambda$ is a metric space we say that they are uniformly $\Gamma$-equivalent if there exist $\left(m_{\varepsilon}^{\lambda}\right)$ such that

$$
\Gamma-\lim _{j}\left(F_{\varepsilon_{j}}^{\lambda_{j}}-m_{\varepsilon_{j}}^{\lambda_{j}}\right)=\Gamma-\lim _{j}\left(G_{\varepsilon_{j}}^{\lambda_{j}}-m_{\varepsilon_{j}}^{\lambda_{j}}\right)
$$

and are proper for all $\lambda_{j} \rightarrow \lambda$ and $\varepsilon_{j} \rightarrow 0$.

Remark 3.1.4 Suppose that $F_{\varepsilon}^{\lambda} \Gamma$-converges to $F^{\lambda}$ and $\left(F_{\varepsilon}^{\lambda}\right)$ and $\left(F^{\lambda}\right)$ are uniformly $\Gamma$ equivalent as above, and that all functionals are equi-coercive and $\Lambda$ is compact. Then we have

$$
\sup _{\lambda \in \Lambda}\left|\inf F_{\varepsilon}^{\lambda}-\min F^{\lambda}\right|=o(1)
$$

or, equivalently, that $f_{\varepsilon}(\lambda)=\inf F_{\varepsilon}^{\lambda}$ converges uniformly to $f(\lambda)=\min F^{\lambda}$ on $\Lambda$. This follows immediately from the fundamental theorem of $\Gamma$-convergence and the compactness of $\Lambda$.

Example 3.1.5 Take $\Lambda=[-1,1]$

$$
F_{\varepsilon}^{\lambda}(u)=\int_{0}^{1}\left(\frac{W(u)}{\varepsilon}+\varepsilon\left|u^{\prime}\right|^{2}\right) d t, \quad \int_{0}^{1} u d t=\lambda
$$

with $W$ as in Example 1.5.4. Then we have for fixed $\lambda$ the $\Gamma$-limit

$$
F^{\lambda}(u)= \begin{cases}0 & \text { if } u(x)=\lambda \\ +\infty & \text { otherwise }\end{cases}
$$

if $\lambda= \pm 1$ and

$$
F^{\lambda}(u)= \begin{cases}c_{W} \#(S(u)) & \text { if } u \in B V((0,1) ;\{ \pm 1\}) \text { and } \int_{0}^{1} u d t=\lambda \\ +\infty & \text { otherwise. }\end{cases}
$$


Note that $f_{\varepsilon}(\lambda)=\inf F_{\varepsilon}^{\lambda}$ is a continuous function, while

$$
f(\lambda)=\min F^{\lambda}= \begin{cases}0 & \text { if }|\lambda|=1 \\ 1 & \text { otherwise }\end{cases}
$$

is not continuous; hence, the convergence $f_{\varepsilon} \rightarrow f$ is not uniform, which implies that $\left(F_{\varepsilon}^{\lambda}\right)$ and $\left(F^{\lambda}\right)$ are not uniformly $\Gamma$-equivalent.

\subsection{A selection criterion}

We use the concept of equivalence as above to formalize a problem of the form: given $F_{\varepsilon}$ find 'simpler' $G_{\varepsilon}$ equivalent to $F_{\varepsilon}$, which capture the 'relevant' features of $F_{\varepsilon}$.

We will proceed as follows:

- compute the $\Gamma$-limit $F$ of $F_{\varepsilon}$. This suggests a limit domain and a class of energies (e.g., energies with sharp interfaces in place of diffuse ones; convex homogeneous functionals in place of oscillating integrals, etc.);

- if the description given by $F$ is not 'satisfactory,' then 'perturb' $F$ so as to obtain a family $\left(G_{\varepsilon}\right)$ $\Gamma$-equivalent to $\left(F_{\varepsilon}\right)$.

The same procedure may apply to parameterized families $\left(F_{\varepsilon}^{\lambda}\right)$.

Of course, the criteria for the construction of $G_{\varepsilon}$ as above may be of different types. In the following example we consider the parameterized family of Example 3.1.5, and the criterion of uniform equivalence.

Example 3.2.1 We consider the functionals $F^{\lambda}$ in Example 3.1.5, which have been shown to be not uniformly equivalent to the sequence $F_{\varepsilon}^{\lambda}$. We wish to construct energies of the same form of $F^{\lambda}$; i.e., with domain $u \in B V((0,1) ;\{ \pm 1\})$ with $\int_{0}^{1} u d t=\lambda$, and uniformly $\Gamma$-equivalent to the sequence $F_{\varepsilon}^{\lambda}$. These energies must then depend on $\varepsilon$. Suppose that $W \in C^{2}$. If we look for energies of the form

$$
G_{\varepsilon}^{\lambda}= \begin{cases}c_{\varepsilon}^{\lambda} \#(S(u)) & \text { if } u \in B V((0,1) ;\{ \pm 1\}) \text { and } \int_{0}^{1} u d x=\lambda \\ +\infty & \text { otherwise, }\end{cases}
$$

then it is possible to show that the choice

$$
c_{\varepsilon}^{\lambda}=\min \left\{\frac{W(\lambda)}{\varepsilon}, c_{W}\right\} .
$$

gives $G_{\varepsilon}^{\lambda}$ uniformly $\Gamma$-equivalent to $F_{\varepsilon}^{\lambda}$. This choice is not unique, even within energies of the form prescribed; in fact we may also take the Taylor expansions of $W$ at \pm 1 in place of $W$

$$
c_{\varepsilon}^{\lambda}=\min \left\{\frac{W^{\prime \prime}(-1)}{2 \varepsilon}(\lambda+1)^{2}, \frac{W^{\prime \prime}(1)}{2 \varepsilon}(\lambda-1)^{2}, c_{W}\right\},
$$


or any other function with the same Taylor expansion. The form of $c_{\varepsilon}^{\lambda}$ highlights that minimizers for $F_{\varepsilon}^{\lambda}$ can either be close to a sharp interface (in which case their value is $c_{W}$ ), or close to the constant $\lambda$ (which gives the energy value $W(\lambda) / \varepsilon$ ). When $\lambda= \pm 1+O(\sqrt{\varepsilon})$ the second type of minimizers may have lower energy. Nevertheless they are never detected by $F^{\lambda}$.

We may also take $G_{\varepsilon}^{\lambda}$ of a slightly more complex form, defined on piecewise-constant functions, setting

$$
G_{\varepsilon}^{\lambda}= \begin{cases}\int_{0}^{1} \frac{W(u)}{\varepsilon} d x+c_{W} \#(S(u)) & \text { if } u \text { piecewise constant and } \int_{0}^{1} u d x=\lambda \\ +\infty & \text { otherwise. }\end{cases}
$$

This choice gives a better description of the minimizers of $F_{\varepsilon}^{\lambda}$.

In the rest of the chapter a 'unsatisfactory description' will mean a partial description of local minimizers. We will then try to perturb the $\Gamma$-limits so as to satisfy this requirement.

\subsection{A 'quantitative' example: phase transitions}

We consider the same type of energies as in Examples 1.5.4 and 3.1.5

$$
F_{\varepsilon}(u)=\int_{0}^{1}\left(\frac{W(u)}{\varepsilon}+\varepsilon\left|u^{\prime}\right|^{2}\right) d x
$$

with $W$ a double-well potential with wells in \pm 1 . For the sake of simplicity, in the present example the domain of $F_{\varepsilon}$ is restricted to 1-periodic functions (i.e., $u$ such that $u(1)=u(0)$ ). This constraint is compatible with the $\Gamma$-limit, which is then given by

$$
F(u)=c_{W} \#(S(u) \cap[0,1)) \quad u \in B V((0,1) ;\{ \pm 1\})
$$

(again, $u$ is extended to a periodic function, so that it may have a jump at 0 , which then is taken into account in the limit energy).

- Note that all functions in $B V((0,1) ;\{ \pm 1\})$ are $L^{1}$-local minimizers (even though not isolated). This is a general fact when we have a lower-semicontinuous function taking discrete values.

- We now show that $F_{\varepsilon}$ has no non-trivial $L^{1}$-local minimizer. We consider the simplified case

$$
W(u)=(|u|-1)^{2} .
$$

In this case $c_{W}=2$. Suppose otherwise that $u$ is a local minimizer. If $u \geq 0$ (equivalently, $u \leq 0)$ then

$$
F_{\varepsilon}(u)=\int_{0}^{1}\left(\frac{(u-1)^{2}}{\varepsilon}+\varepsilon\left|u^{\prime}\right|^{2}\right) d t .
$$


Since this functional is convex, its only local minimizer is the global minimizer $u=1$. Otherwise, we can suppose, up to a translation, that there exists $L \in(0,1)$ such that $u( \pm L / 2)=0$ and $u(x)>0$ for $|x|<L / 2$. Again, using the convexity of

$$
F_{\varepsilon}^{L}(u)=\int_{-L / 2}^{L / 2}\left(\frac{(u-1)^{2}}{\varepsilon}+\varepsilon\left|u^{\prime}\right|^{2}\right) d x
$$

we conclude that $u$ must be the global minimizer of $F_{L}$ with zero boundary data; i.e., the solution of

$$
\left\{\begin{array}{l}
u^{\prime \prime}=\frac{1}{\varepsilon^{2}}(u-1) \\
u\left( \pm \frac{L}{2}\right)=0
\end{array}\right.
$$

This gives

$$
u(x)=1-\left(\cosh \left(\frac{L}{2 \varepsilon}\right)\right)^{-1} \cosh \left(\frac{x}{\varepsilon}\right)
$$

and

$$
F_{\varepsilon}^{L}(u)=\frac{\sinh \left(\frac{L}{\varepsilon}\right)}{\left(\cosh \left(\frac{L}{2 \varepsilon}\right)\right)^{2}}
$$

Note that

$$
\frac{d^{2}}{d L^{2}} F_{\varepsilon}^{L}(u)=-\frac{1}{\varepsilon^{2}} \frac{\sinh \left(\frac{L}{2 \varepsilon}\right)}{\left(\cosh \left(\frac{L}{2 \varepsilon}\right)\right)^{3}}
$$

i.e., this minimum value is a concave function of $L$. This immediately implies that no local minimizer may exist with changing sign; in fact, such a minimizer would be a local minimizer of the function

$$
f\left(L_{1}, \ldots, L_{K}\right)=\sum_{k=1}^{K} \frac{\sinh \left(\frac{L_{k}}{\varepsilon}\right)}{\left(\cosh \left(\frac{L_{k}}{2 \varepsilon}\right)\right)^{2}},
$$

for some $K>0$ under the constraint $L_{k}>0$ and $\sum_{k} L_{k}=1$, which is forbidden by the negative definiteness of its Hessian matrix. Note moreover that

$$
F_{\varepsilon}^{L}(u)=2-4 e^{-\frac{L}{\varepsilon}}+O\left(e^{-\frac{2 L}{\varepsilon}}\right)
$$

and that $-4 e^{-\frac{L}{\varepsilon}}$ is still a concave function of $L$.

- We can now propose a 'correction' to $F$ by considering in its place

$$
G_{\varepsilon}(u)=c_{W} \#(S(u))-\sum_{x \in S(u) \cap[0,1)} 4 e^{-\frac{1}{\varepsilon} \mid x-\max (S(u) \cap(-\infty, x) \mid}
$$


defined on periodic functions with $u \in B V((0,1) ;\{ \pm 1\})$. It is easily seen that $G_{\varepsilon} \Gamma$ converges to $F$, and is hence equivalent to $F_{\varepsilon}$; thanks to the concavity of the second term the same argument as above shows that we have no non-trivial local minimizers. As a side remark note that this approximation also maintains the stationary points of $F_{\varepsilon}$, which are functions with $K$ jumps at distance $1 / K$. This is easily seen after remarking that the distances between consecutive points must be a stationary point for (3.2). Moreover, the additional terms can also be computed as a development by $\Gamma$-convergence, which extends this equivalence to 'higher order'.

Remark 3.3.1 (interaction with the boundary) The case without periodicity conditions can be reduced to the case with periodic boundary conditions upon remarking that if $\widetilde{u}$ denotes the even extension of $u$ to $(-1,1)$ then

$$
F_{\varepsilon}(u)=\frac{1}{2} \int_{-1}^{1}\left(\frac{W(\widetilde{u})}{\varepsilon}+\varepsilon\left|\widetilde{u}^{\prime}\right|^{2}\right) d x
$$

and $\widetilde{u}$ satisfies periodic boundary conditions. We can then apply the arguments above to obtain that if $x_{1}<\cdots<x_{n}$ denote the location of points in $(0,1)$ where $u$ changes sign as in the computation above (where $L_{k}=x_{k}-x_{k-1}$ ), then we have the corresponding estimate

$$
\begin{aligned}
F_{\varepsilon}(u) & \geq \sum_{k=2}^{n} \frac{\sinh \left(\frac{x_{k}-x_{k-1}}{\varepsilon}\right)}{\left(\cosh \left(\frac{x_{k}-x_{k-1}}{2 \varepsilon}\right)\right)^{2}}+\frac{\sinh \left(\frac{2 x_{1}}{\varepsilon}\right)}{2\left(\cosh \left(\frac{2 x_{1}}{2 \varepsilon}\right)\right)^{2}}+\frac{\sinh \left(\frac{2-2 x_{n}}{\varepsilon}\right)}{2\left(\cosh \left(\frac{2-2 x_{n}}{2 \varepsilon}\right)\right)^{2}} \\
& =\sum_{k=2}^{n} \frac{\sinh \left(\frac{x_{k}-x_{k-1}}{\varepsilon}\right)}{\left(\cosh \left(\frac{x_{k}-x_{k-1}}{2 \varepsilon}\right)\right)^{2}}+\tanh \left(\frac{x_{1}}{\varepsilon}\right)+\tanh \left(\frac{1-x_{n}}{\varepsilon}\right) .
\end{aligned}
$$

The last two terms represent the interaction of the points $x_{0}$ and $x_{n}$ with the boundary of $(0,1)$. The 'corrected' $\Gamma$-converging sequence in this case can be written as

$$
G_{\varepsilon}(u)=c_{W} \#(S(u))-\sum_{k=2}^{n} 4 e^{-\frac{1}{\varepsilon}\left|x_{k}-x_{k-1}\right|}-2 e^{-\frac{2}{\varepsilon} x_{1}}-2 e^{-\frac{2}{\varepsilon}\left(1-x_{n}\right)}
$$

if $S(u)=\left\{x_{1}, \ldots, x_{n}\right\}$ with $x_{k}<x_{k-1}$.

\subsection{A 'qualitative' example: Lennard-Jones atomistic sys- tems}

As in Example 1.5.5, we consider a scaled systems of one-dimensional nearest-neighbour atomistic interactions through a Lennard-Jones type interaction. Let $J$ be a $C^{2}$ potential 
as in Figure 3.1, with domain $(-1,+\infty)$ (we set $J(w)=+\infty$ for $w \leq-1$ ), minimum in 0 with $J^{\prime \prime}(0)>0$, convex in $\left(-1, w_{0}\right)$, concave in $\left(w_{0},+\infty\right)$ and tending to $J(\infty)<+\infty$ at $+\infty$. We consider the energy

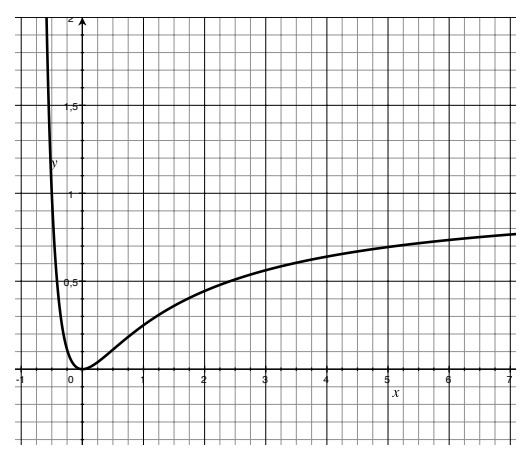

Figure 3.1: a (translation of a) Lennard-Jones potential

$$
F_{\varepsilon}^{\lambda}(u)=\sum_{i=1}^{N} J\left(\frac{u_{i}-u_{i-1}}{\sqrt{\varepsilon}}\right)
$$

with the boundary conditions $u_{0}=0$ and $u_{N}=\lambda \geq 0$. Here $\varepsilon=1 / N$ with $N \in \mathbb{N}$. The vector $\left(u_{0}, \ldots, u_{N}\right)$ is identified with a discrete function defined on $\varepsilon \mathbb{Z} \cap[0,1]$ or with its piecewise-affine interpolation. With this last identification, $F_{\varepsilon}^{\lambda}$ can be viewed as functionals in $L^{1}(0,1)$, and their $\Gamma$-limit computed with respect to that topology.

Taking into account the boundary conditions, we can extend all functions to $u(x)=0$ for $x \leq 0$ and $u(x)=\lambda$ for $x \geq \lambda$, and denote by $S(u)$ (set of discontinuity points of $u$ ) the minimal set such that $u \in H^{1}((-s, 1+s) \backslash S(u))$ for $s>0$. With this notation, the same arguments as in Example 1.5.5 give that the $\Gamma$-limit is defined on piecewise- $H^{1}(0,1)$ functions by

$$
F^{\lambda}(u)=\frac{1}{2} J^{\prime \prime}(0) \int_{0}^{1}\left|u^{\prime}\right|^{2} d t+J(\infty) \#(S(u) \cap[0,1])
$$

with the constraint that $u^{+}>u^{-}$on $S(u)$ and the boundary conditions $u^{-}(0)=0, u^{+}(1)=$ $\lambda$ (so that $S(u)$ is understood to contain also 0 or 1 if $u^{+}(0)>0$ or $u-(1)<\lambda$ ). For simplicity of notation we suppose

$$
\frac{1}{2} J^{\prime \prime}(0)=J(\infty)=1
$$

- Local minimizers of $F^{\lambda}$. By the strict convexity of $\int_{0}^{1}\left|u^{\prime}\right|^{2} d t$ this part of the energy is minimized, given the average $z=\int_{0}^{1} u^{\prime} d t$, by the piecewise-constant gradient $u^{\prime}=z$. From now on we tacitly assume that $u^{\prime}$ is constant. We then have two cases depending on the number of jumps: 
(i) if $S(u)=\emptyset$ then $z=\lambda$, and this is a strict local minimizer since any $L^{1}$ perturbation with a jump of size $w$ and (average) gradient $z$ has energy $z^{2}+1$ independent of $w$, which is strictly larger than $\lambda^{2}$ if the perturbation is small;

(ii) if $\# S(u) \geq 1$ then $L^{1}$ local minimizers are all functions with $u^{\prime}=0$ (since otherwise we can strictly decrease the energy by taking a small perturbation $v$ with the same set of discontinuity points and $v^{\prime}=s u^{\prime}$ with $s<1$ ).

The energy of the local minima in dependence of $\lambda$ is pictured in Figure 3.2.

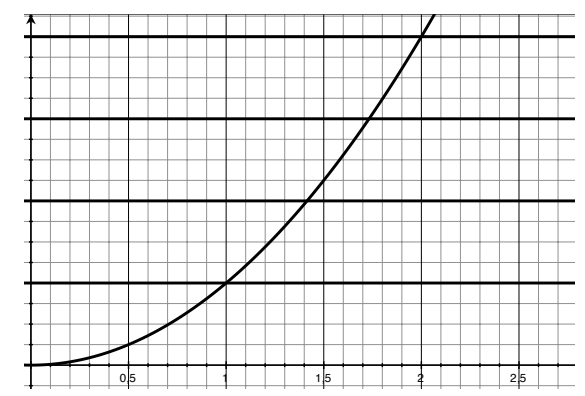

Figure 3.2: local minima for $F^{\lambda}$

- Local minimizers of $F_{\varepsilon}^{\lambda}$. This is a finite-dimensional problem, whose stationarity condition is

$$
J^{\prime}\left(\frac{u_{i}-u_{i-1}}{\sqrt{\varepsilon}}\right)=\sigma \quad \text { for all } i,
$$

for some $\sigma>0$. The shape of $J^{\prime}$ is pictured in Figure 3.3; its maximum is achieved for $w=w_{0}$. Note that for all $0<\sigma<J^{\prime}\left(w_{0}\right)$ we have two solutions of $J^{\prime}(w)=\sigma$, while we have no solution for $\sigma>J^{\prime}\left(w_{0}\right)$.

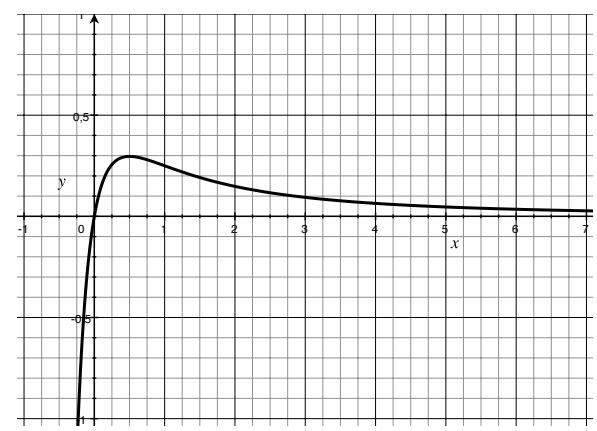

Figure 3.3: derivative of $J$

We have three cases:

(i) we have

$$
\frac{u_{i}-u_{i-1}}{\sqrt{\varepsilon}} \leq w_{0}
$$


for all $i$. In this case the boundary condition gives $\frac{u_{i}-u_{i-1}}{\varepsilon}=\lambda$ for all $i$, so that we have the constraint.

$$
\lambda \leq \frac{w_{0}}{\sqrt{\varepsilon}}
$$

This solution is a local minimum. This is easily checked when $\lambda<\frac{w_{0}}{\sqrt{\varepsilon}}$ since small perturbations maintain the condition (3.3). In the limit case $\lambda=\frac{w_{0}}{\sqrt{\varepsilon}}$ we may consider only perturbations where (3.3) is violated at exactly one index (see (ii) below), to which there corresponds an energy

$$
J\left(w_{0}+t\right)+(N-1) J\left(w_{0}-\frac{t}{N-1}\right)
$$

for $t \geq 0$, which has a local minimum at 0 .

(ii) condition (3.3) is violated by two (or more) indices $j$ and $k$. Let $\bar{w}$ be such that

$$
\frac{u_{j}-u_{j-1}}{\sqrt{\varepsilon}}=\frac{u_{k}-u_{k-1}}{\sqrt{\varepsilon}}=\bar{w}>w_{0} .
$$

We may perturb $u_{i}-u_{i-1}$ only for $i=j, k$, so that the energy varies by

$$
f(s):=J(\bar{w}+s)+J(\bar{w}-s)-2 J(\bar{w}) .
$$

We have $f^{\prime}(0)=0$ and $f^{\prime \prime}(0)=2 J^{\prime \prime}(\bar{w})<0$, which contradicts the minimality of $u$.

(iii) condition (3.3) is violated exactly by one index. The value of $w=\frac{u_{i}-u_{i-1}}{\sqrt{\varepsilon}}$ for the $N-1$ indices satisfying (3.3) is obtained by computing local minimizers of the energy on such functions, which is

$$
f_{\varepsilon}^{\lambda}(w):=(N-1) J(w)+J\left(\frac{\lambda}{\sqrt{\varepsilon}}-(N-1) w\right)
$$

defined for $0 \leq w \leq \min \left\{w_{0}, \frac{1}{N-1}\left(\frac{\lambda}{\sqrt{\varepsilon}}-w_{0}\right)\right\}$. We compute

$$
\left(f_{\varepsilon}^{\lambda}\right)^{\prime}(w):=(N-1)\left(J^{\prime}(w)-J^{\prime}\left(\frac{\lambda}{\sqrt{\varepsilon}}-(N-1) w\right)\right) \text {. }
$$

Note that

$$
f_{\varepsilon}^{\lambda}(0)=J\left(\frac{\lambda}{\sqrt{\varepsilon}}\right)=1-o(1)
$$

and $\left(f_{\varepsilon}^{\lambda}\right)^{\prime}(0)<0$. If $\lambda>w_{0} / \sqrt{\varepsilon}$ then $\left(f_{\varepsilon}^{\lambda}\right)^{\prime}(w)=0$ has a unique solution, which is a local minimizer, while if $\lambda \leq w_{0} / \sqrt{\varepsilon}$ we have two solutions $w_{1}<w_{2}$, of which the first one is a local minimizer. We then have a unique curve of local minimizers with one jump.

The energy of the local minima in dependence of $\lambda$ is schematically pictured in Fig. 3.4. 


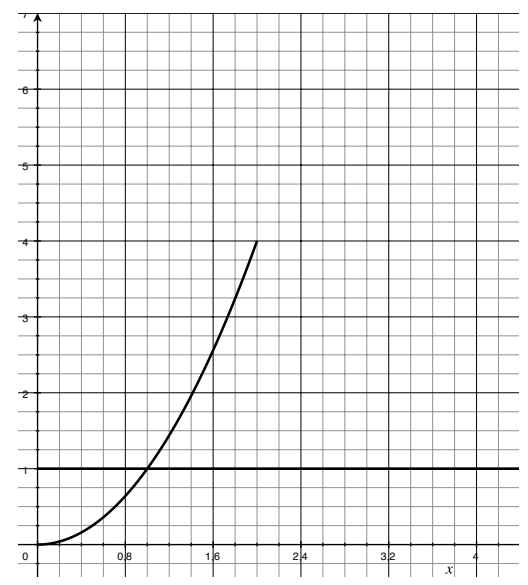

Figure 3.4: local minima for $F_{\varepsilon}^{\lambda}$

- A qualitative comparison of local minimization. First, the local minimizer for $F_{\varepsilon}^{\lambda}$ which never exceeds the convexity threshold (corresponding to the minimizer with $S(u)=\emptyset$ for $F^{\lambda}$ ) exists only for $\lambda \leq w_{0} / \sqrt{\varepsilon}$; second, we only have one curve of local minimizers for $F_{\varepsilon}^{\lambda}$ which exceed the convexity threshold for only one index (corresponding to the minimizers with $\# S(u)=1$ for $\left.F^{\lambda}\right)$.

- $\Gamma$-equivalent energies. We choose to look for energies defined on piecewise- $H^{1}$ functions of the form

$$
G_{\varepsilon}^{\lambda}(u)=\int_{0}^{1}\left|u^{\prime}\right|^{2} d t+\sum_{t \in S(u)} g\left(\frac{u^{+}-u^{-}}{\sqrt{\varepsilon}}\right),
$$

again with the constraint that $u^{+}>u^{-}$on $S(u)$ and the boundary conditions $u^{-}(0)=$ $0, u^{+}(1)=\lambda$. In order that local minimizers satisfy $\#(S(u)) \leq 1$ we require that $g$ : $(0,+\infty) \rightarrow(0,+\infty)$ be strictly concave. In fact, with this condition the existence of two points in $S(u)$ is ruled out by noticing that given $w_{1}, w_{2}>0$ the function $t \mapsto g\left(w_{1}+t\right)+$ $g\left(w_{2}-t\right)$ is concave. Moreover, we also require that $g$ satisfy

$$
\lim _{w \rightarrow+\infty} g(w)=1 .
$$

With this condition is is easily seen that we have the $\Gamma$-convergence of $G_{\varepsilon}^{\lambda}$ to $F^{\lambda}$.

In order to make a comparison with the local minimizers of $F_{\varepsilon}^{\lambda}$ we first consider local minimizers with $S(u)=\emptyset$; i.e., $u(t)=\lambda t$. Such a function is a local minimizer if it is not energetically favourable to introduce a small jump of size $w$; i.e., if 0 is a local minimizer for

$$
g_{\varepsilon}^{\lambda}(w):=(\lambda-w)^{2}+g\left(\frac{w}{\sqrt{\varepsilon}}\right),
$$

where we have extended the definition of $g$ by setting $g(0)=0$. Note that if $g$ is not continuous in 0 then 0 is a strict local minimizer for $g_{\varepsilon}^{\lambda}$ for all $\lambda$. Otherwise, we can 
compute the derivative, and obtain that

$$
\frac{d}{d w} g_{\varepsilon}^{\lambda}(0)=-2 \lambda+\frac{1}{\sqrt{\varepsilon}} g^{\prime}(0)
$$

For $\varepsilon$ small enough, 0 is a (isolated) local minimizer if and only if $\frac{d}{d w} g_{\varepsilon}^{\lambda}(0)>0$; i.e.,

$$
\lambda<\frac{1}{2 \sqrt{\varepsilon}} g^{\prime}(0) .
$$

If we choose

$$
g^{\prime}(0)=2 w_{0}
$$

we obtain the desired constraint on this type of local minimizers. A possible simple choice of $g$ is

$$
g(w)=\frac{2 w_{0} w}{1+2 w_{0} w} .
$$

We finally consider local minimizers with $\#(S(u))=1$. If $w$ denotes the size of the jump then again computing the derivative of the energy, we conclude the existence of a single local minimizer $w$ with

$$
2(\lambda-w)=\frac{1}{\sqrt{\varepsilon}} g^{\prime}\left(\frac{w}{\sqrt{\varepsilon}}\right),
$$

and energy approaching 1 as $\varepsilon \rightarrow 0$.

- With the choice above the pictures of the local minimizers for $G_{\varepsilon}^{\lambda}$ and for $F_{\varepsilon}^{\lambda}$ are of the same type, but may vary in quantitative details. We have not addressed the problem of the uniformity of this description, for which a refinement of the choice of $g$ could be necessary.

- As a conclusion, we remark that this example has some modeling implications. The functional $F^{\lambda}$ can be seen as a one-dimensional version of the energy of a brittle elastic medium according to Griffith's theory of Fracture $(S(u)$ represents the fracture site in the reference configuration), which is then interpreted as a continuum approximation of an atomistic model with Lennard Jones interactions. The requirement that also local minima may be reproduced by the limit theory has made us modify our functional $F^{\lambda}$ obtaining another sequence of energies, which maintain an internal parameter $\varepsilon$. Energies of the form $G_{\varepsilon}^{\lambda}$ are present in the literature, and are related to Barenblatt's theory of ductile Fracture. Note that in all these considerations the parameter $\lambda$ appears in the functionals only as a boundary condition, and does not influence the form of the energy.

\subsection{A negative example: oscillating perimeters}

The procedure described above cannot be always performed in a simple fashion. This may happen if the structure of the $\Gamma$-limit $F$ cannot be easily modified to follow the pattern of 
the local minimizers of $F_{\varepsilon}$. We include an example where local minimizers if $F_{\varepsilon}$ tend to be a dense set, while functionals with the structure of $F$ have no local minimizers.

Example 3.5.1 We consider the function $a: \mathbb{Z}^{2} \rightarrow\{1,2\}$

$$
a\left(x_{1}, x_{2}\right)= \begin{cases}1 & \text { if } x_{1} \in \mathbb{Z} \text { or } x_{2} \in \mathbb{Z} \\ 2 & \text { otherwise }\end{cases}
$$

and the related scaled-perimeter functionals

$$
F_{\varepsilon}(A)=\int_{\partial A} a\left(\frac{x}{\varepsilon}\right) d \mathcal{H}^{1}
$$

defined on Lipschitz sets $A$. The energies $F_{\varepsilon} \Gamma$-converge, with respect to the convergence $A_{\varepsilon} \rightarrow A$, understood as the $L^{1}$ convergence of the corresponding characteristic functions, to an energy of the form

$$
F(A)=\int_{\partial^{*} A} g(\nu) d \mathcal{H}^{1}
$$

defined on all sets of finite perimeter ( $\nu$ denotes the normal to $\partial^{*} A$ ). A direct computation shows that actually

$$
g(\nu)=\|\nu\|_{1}=\left|\nu_{1}\right|+\left|\nu_{2}\right| .
$$

Furthermore, it is easily seen that the same $F$ is equivalently the $\Gamma$-limit of

$$
\widetilde{F}_{\varepsilon}(A)=\mathcal{H}^{1}(\partial A)
$$

defined on $A$ which are the union of cubes $Q_{i}^{\varepsilon}:=\varepsilon\left(i+(0,1)^{2}\right)$ with $i \in \mathbb{Z}^{2}$. We denote by $\mathcal{A}_{\varepsilon}$ the family of such $A$. Note that $\widetilde{F}_{\varepsilon}$ is the restriction of $F_{\varepsilon}$ to $\mathcal{A}_{\varepsilon}$.

If $A \in \mathcal{A}_{\varepsilon}$ then $A$ is trivially a $L^{1}$-local minimizer for $\widetilde{F}_{\varepsilon}$ with $\delta<\varepsilon^{2}$, since any two distinct elements of $\mathcal{A}_{\varepsilon}$ are at least at $L^{1}$-distance $\varepsilon^{2}$ (the area of a single $\varepsilon$-square). It can be proved also that all $A \in \mathcal{A}_{\varepsilon}$ are $L^{1}$-local minimizer for $F_{\varepsilon}$ with $\delta=C \varepsilon^{2}$ for $C>0$ sufficiently small.

\subsection{References to Chapter 3}

The notion of equivalence by $\Gamma$-convergence is introduced and analyzed in the paper by Braides and Truskinovsky [18].

Local minimizers for Lennard-Jones type potentials (also with external forces) are studied in the paper by Braides, Dal Maso and Garroni [12]

More details on the derivation of fracture energies from interatomic potentials and the explanation of the $\sqrt{\varepsilon}$-scaling can be found in the paper by Braides, Lew and Ortiz [16] (see also the quoted paper by Braides and Truskinovsky for an explanation in terms of uniform $\Gamma$-equivalence).

For general reference on sets of finite perimeter and BV functions we refer to $[10,5,33]$ 


\section{Chapter 4}

\section{Convergence of local minimizers}

In this section we consider a generalization of the fundamental theorem of $\Gamma$-convergence when we have strict local minimizers of the $\Gamma$-limit.

\subsection{Convergence to isolated local minimizers}

The following theorem shows that we may extend (part of) the fundamental theorem of $\Gamma$-convergence to isolated local minimizers of the $\Gamma$-limit $F$; i.e. (we suppose that $F$ be defined on a metric space with distance $d$ ), to points $u_{0}$ such that there exists $\delta>0$ such that

$$
F\left(u_{0}\right)<F(u) \quad \text { if } \quad 0<d\left(u, u_{0}\right) \leq \delta .
$$

The proof of this theorem essentially consists in remarking that we may at the same time apply Proposition 1.1.2 (more precisely, Remark 1.1.4) to the closed ball of center $u_{0}$ and radius $\delta$, and Proposition 1.1.6 to the open ball of center $u_{0}$ and radius $\delta$.

Theorem 4.1.1 Suppose that each $F_{\varepsilon}$ is coercive and lower semicontinuous and the sequence $\left(F_{\varepsilon}\right) \Gamma$-converge to $F$ and is equicoercive. If $u_{0}$ is an isolated local minimizer of $F$ then there exist a sequence $\left(u_{\varepsilon}\right)$ converging to $u_{0}$ with $u_{\varepsilon}$ a local minimizer of $F_{\varepsilon}$ for $\varepsilon$ small enough.

Proof. Let $\delta>0$ satisfy (4.1). Note that by the coerciveness and lower semicontinuity of $F_{\varepsilon}$ there exists a minimizer $u_{\varepsilon}$ of $F_{\varepsilon}$ on $\overline{B_{\delta}\left(u_{0}\right)}$, the closure of $B_{\delta}\left(u_{0}\right)=\left\{u: d\left(u, u_{0}\right) \leq \delta\right\}$. By the equicoerciveness of $\left(F_{\varepsilon}\right)$, upon extracting a subsequence, we can suppose that $u_{\varepsilon} \rightarrow \bar{u}$. Since $\bar{u} \in \overline{B_{\delta}\left(u_{0}\right)}$ we then have

$$
\begin{aligned}
F\left(u_{0}\right) & \leq F(\bar{u}) \leq \liminf _{\varepsilon \rightarrow 0} F_{\varepsilon}\left(u_{\varepsilon}\right)=\liminf _{\varepsilon \rightarrow 0} \frac{\min }{B_{\delta}\left(u_{0}\right)} F_{\varepsilon} \\
& \leq \limsup _{\varepsilon \rightarrow 0} \inf _{B_{\delta}\left(u_{0}\right)} F_{\varepsilon} \leq \inf _{B_{\delta}\left(u_{0}\right)} F=F\left(u_{0}\right),
\end{aligned}
$$


where we have used Proposition 1.1.6 in the last inequality. By (4.1) we have that $\bar{u}=u_{0}$ and $u_{\varepsilon} \in B_{\delta}\left(u_{0}\right)$ for $\varepsilon$ small enough, which proves the thesis.

Remark 4.1.2 In the theorem above it is sufficient to require the coerciveness properties for $F_{\varepsilon}$ only on bounded sets, since they are applied to minimization problems on $\overline{B_{\delta}\left(u_{0}\right)}$.

Remark 4.1.3 Clearly, the existence of an isolated (local) minimizer in the limit does not imply that the converging (local) minimizers are isolated. It suffices to consider $F_{\varepsilon}(x)=$ $((x-\varepsilon) \vee 0)^{2}$ converging to $F(x)=x^{2}$.

Remark 4.1.4 In Section 3.4 we have noticed that the limit fracture energy $F^{\lambda}$ possesses families of $L^{1}$-local minimizers with an arbitrary number of jump points, while the approximating functionals $F_{\varepsilon}^{\lambda}$ have local minimizers corresponding to limit functions with only one jump point. This cannot directly be deduced from the result above since those limit local minimizers are not isolated. Anyhow $L^{1}$-local minimizers with one jump are strict local minimizers with respect to the distance

$$
\begin{aligned}
d(u, v)= & \int_{0}^{1}|u-v| d x+\sum_{x \in(0,1)}\left|\left(u^{+}-u^{-}\right)-\left(v^{+}-v^{-}\right)\right| \\
= & \int_{0}^{1}|u-v| d x+\sum_{x \in S(u) \cap S(v)}\left|\left(u^{+}-u^{-}\right)-\left(v^{+}-v^{-}\right)\right| \\
& +\sum_{x \in S(u) \backslash S(v)}\left|u^{+}-u^{-}\right|+\sum_{x \in S(v) \backslash S(u)}\left|v^{+}-v^{-}\right|,
\end{aligned}
$$

which penalizes (large) jumps of a competitor $v$ outside $S(u)$. Upon suitably defining interpolations of discrete functions in $S B V(0,1)$ (where jumps correspond to difference quotients above the threshold $w_{0} / \sqrt{\varepsilon}$ ) it can be shown that the $\Gamma$-limit remains unchanged with this convergence, so that we may apply Theorem 4.1.1. Note that for discrete functions the notion of local minimizers is the same as for the $L^{1}$-distance since we are in a finitedimensional space. Note moreover that $L^{1}$-local minimizers of $F^{\lambda}$ with more than one jump are not strict local minimizers for the distance $d$ above. Indeed, if $u^{\prime}=0$ and $S(u)=\left\{x_{1}, \ldots, x_{N}\right\}$ with $0 \leq x_{1}<\cdots<x_{N}$ and $N \geq 2$, then any $u_{s}=u+s \chi_{\left(x_{1}, x_{2}\right)}$ is still a local minimizer for $F^{\lambda}$ with $F^{\lambda}\left(u_{s}\right)=F^{\lambda}(u)=N$ and $d\left(u, u_{s}\right)=s\left(1+\left|x_{2}-x_{1}\right|\right)$.

\subsection{Two examples}

We use Theorem 4.1.1 to prove the existence of sequences of converging local minima. 
Example 4.2.1 (local minimizers for elliptic homogenization) Consider the functionals in Example 1.4.2. Suppose furthermore that $\bar{g}$ has an isolated local minimum at $z_{0}$. We will show that the constant function $u_{0}(x)=z_{0}$ is a $L^{1}$-local minimizer of $F_{\text {hom }}+G$. Thanks to Theorem 4.1.1 we then deduce that there exists a sequence of local minimizers of $F_{\varepsilon}+G_{\varepsilon}$ (in particular, if $g$ is differentiable with respect to $u$, of solutions of the Euler-Lagrange equation (1.15)) converging to $u_{0}$.

We only prove the statement in the one-dimensional case, for which $\Omega=(0, L)$. We now consider $\delta>0$ and $u$ such that

$$
\left\|u-u_{0}\right\|_{L^{1}(0, L)} \leq \delta
$$

Since $z_{0}$ is an isolated local minimum of $\bar{g}$ there exists $h>0$ such that $g\left(z_{0}\right)<g(z)$ if $0<\left|z-z_{0}\right| \leq h$. If $\left\|u-u_{0}\right\|_{\infty} \leq h$ then $G(u) \geq G\left(u_{0}\right)$ with equality only if $u=u_{0}$ a.e., so that the thesis is verified. Suppose otherwise that there exists a set of positive measure $A$ such that $\left|u-u_{0}\right|>h$ on $A$. We then have

$$
h|A| \leq \int_{A}\left|u-u_{0}\right| d t \leq \delta
$$

so that $|A| \leq \delta / h$. We can then estimate

$$
G(u) \geq \min \bar{g}|A|+(L-|A|) \bar{g}\left(z_{0}\right) \geq G\left(u_{0}\right)-\frac{\bar{g}\left(z_{0}\right)-\min \bar{g}}{h} \delta .
$$

On the other hand, there exists a set of positive measure $B$ such that

$$
\left|u(x)-u_{0}\right| \leq \frac{\delta}{L}
$$

(otherwise the $L^{1}$ estimate doe not hold). Let $x_{1} \in B$ and $x_{2} \in A$, we can estimate (we can assume $\left.x_{1}<x_{2}\right)$

$$
F_{\text {hom }}(u) \geq \alpha \int_{\left[x_{1}, x_{2}\right]}\left|u^{\prime}\right|^{2} d t \geq \alpha \frac{\left(u\left(x_{2}\right)-u\left(x_{1}\right)\right)^{2}}{x_{2}-x_{1}} \geq \alpha \frac{\left(h-\frac{\delta}{L}\right)^{2}}{L}
$$

(using Jensen's inequality). Summing up we have

$$
\begin{aligned}
F_{\text {hom }}(u)+G(u) & \geq F_{\text {hom }}\left(u_{0}\right)+G\left(u_{0}\right)+\alpha \frac{\left(h-\frac{\delta}{L}\right)^{2}}{L}-\frac{\bar{g}\left(z_{0}\right)-\min \bar{g}}{h} \delta \\
& =F_{\text {hom }}\left(u_{0}\right)+G\left(u_{0}\right)+\alpha \frac{h^{2}}{L}+O(\delta) \\
& >F_{\text {hom }}\left(u_{0}\right)+G\left(u_{0}\right)
\end{aligned}
$$

for $\delta$ small as desired. 
Example 4.2.2 (Kohn-Sternberg) In order to prove the existence of $L^{1}$ local minimizers for the energies $F_{\varepsilon}$ in (1.18) by Theorem 1.4.2 it suffices to prove the existence of isolated local minimizers for the minimal interface problem related to the energy (1.20). In order for this to hold we need some hypothesis on the set $\Omega$ (for example, it can be proved that no non-trivial local minimizer exists when $\Omega$ is convex).

We treat the two-dimensional case only. We suppose that $\Omega$ is bounded, regular, and has an 'isolated neck'; i.e., it contains a straight segment whose endpoints meet $\partial \Omega$ perpendicularly, and $\partial \Omega$ is strictly concave at those endpoints (see Fig. 4.1). We will show

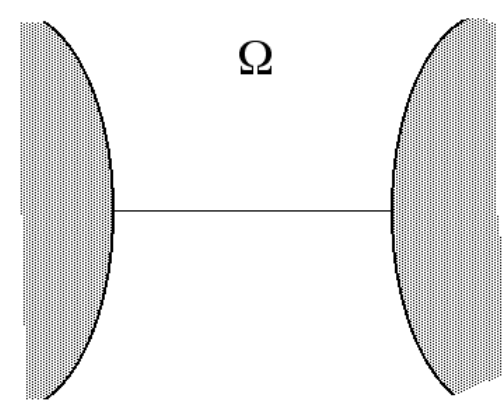

Figure 4.1: a neck in the open set $\Omega$

that the set with boundary that segment (we can suppose that the segment disconnets $\Omega$ ) is an isolated local minimizer for the perimeter functional.

We can think that the segment is $(0, L) \times\{0\}$. By the strict concavity of $\partial \Omega$ there exist $h>0$ such that in a rectangular neighbourhood of the form $(a, b) \times(-2 h, 2 h)$ the lines $x=0$ and $x=L$ meet $\partial \Omega$ only at $(0,0)$ and $(0, L)$ respectively. The candidate strict local minimizer is $A_{0}=\{(x, y) \in \Omega ; x>0\}$, which we identify with the function $u_{0}=-1+2 \chi_{A_{0}}$, taking the value +1 in $A_{0}$ and -1 in $\Omega \backslash A_{0}$.

Take another test set $A$. The $L^{1}$ closeness condition for functions translates into

$$
\left|A \triangle A_{0}\right| \leq \delta
$$

We may suppose that $A$ is sufficiently regular (some minor extra care must be taken when $A$ is a set of finite perimeter, but the proof may be repeated essentially unchanged).

Consider first the case that $A$ contains a horizontal segment $y=M$ with $M \in[h, 2 h]$ and its complement contains a horizontal segment $y=m$ with $m \in[-2 h, h]$. Then a portion of the boundary $\partial A$ is contained in the part of $\Omega$ in the strip $|y| \leq 2 h$, and its length is strictly greater than $L$, unless it is exactly the minimal segment (see Fig. 4.2).

If the condition above is not satisfied then $A$ must not contain, e.g., any horizontal segment $y=t$ with $t \in[h, 2 h]$ (see Fig. 4.3). In particular, the length of the portion of $\partial A$ contained with $h \leq y \leq 2 h$ is not less than $h$. Consider now the one-dimensional set

$$
B=\{t \in(0, L): \partial A \cap(\{t\} \times(-h, h))=\emptyset\} .
$$




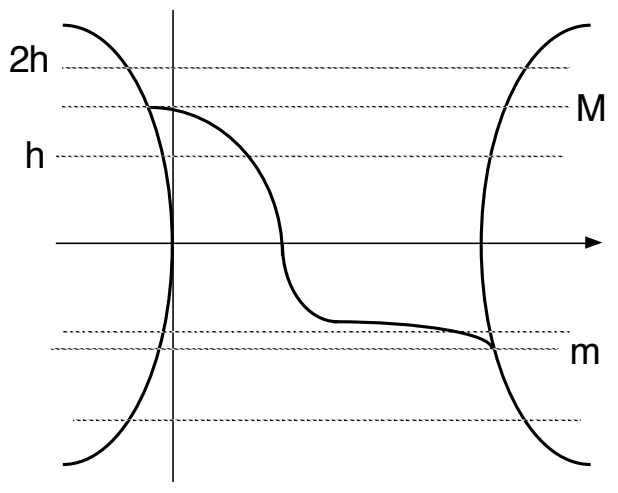

Figure 4.2: comparison with a uniformly close test set

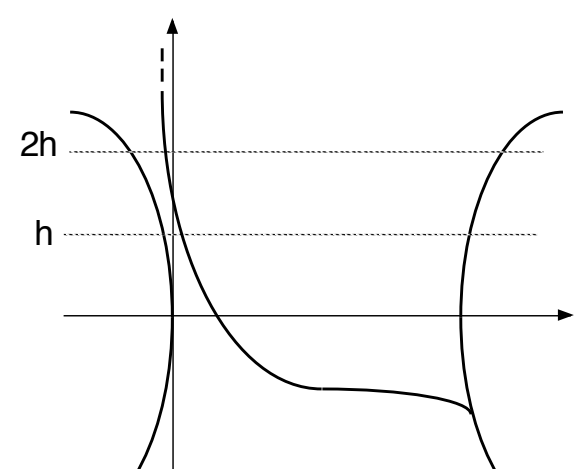

Figure 4.3: comparison with a $L^{1}$-close test set

We have

$$
\delta \geq\left|A \triangle A_{0}\right| \geq h|B|,
$$

so that $|B| \leq \delta / h$, and the portion of $\partial A$ with $h \leq y \leq 2 h$ is not less than $L-\delta / h$. Summing up we have

$$
\mathcal{H}^{1}(\partial A) \geq h+L-\frac{\delta}{h}=\mathcal{H}^{1}\left(\partial A_{0}\right)+h-\frac{\delta}{h},
$$

and the desired strict inequality for $\delta$ small enough.

\subsection{Generalizations}

We can give some generalizations of Theorem 4.1.1 in terms of scaled energies. 
Proposition 4.3.1 Let $F_{\varepsilon}$ satisfy the coerciveness and lower-semicontinuity assumptions of Theorem 4.1.1. Suppose furthermore that a bounded positive function $f:(0,+\infty) \rightarrow$ $(0,+\infty)$ exists and constants $m_{\varepsilon}$ such that the scaled functionals

$$
\widetilde{F}_{\varepsilon}(u)=\frac{F_{\varepsilon}(u)-m_{\varepsilon}}{f(\varepsilon)}
$$

are equicoercive and $\Gamma$-converge on $\overline{B_{\delta}\left(u_{0}\right)}$ to $\widetilde{F}_{0}$ given by

$$
\widetilde{F}_{0}(u)= \begin{cases}0 & \text { if } u=u_{0} \\ +\infty & \text { otherwise }\end{cases}
$$

in $\overline{B_{\delta}\left(u_{0}\right)}$. Then there exists a sequence $\left(u_{\varepsilon}\right)$ converging to $u_{0}$ of local minimizers of $F_{\varepsilon}$.

Remark 4.3.2 (i) First note that the functionals $F_{\varepsilon}$ in Theorem 4.1.1 satisfy the hypotheses of the above proposition, taking, e.g., $f(\varepsilon)=\varepsilon$ and $m_{\varepsilon}$ equal to the minimum of $F_{\varepsilon}$ in $\overline{B_{\delta}\left(u_{0}\right)}$;

(ii) Note that the hypothesis above is satisfied if there exist constants $m_{\varepsilon}$ such that

(a) $\Gamma-\limsup _{\varepsilon \rightarrow 0}\left(F_{\varepsilon}\left(u_{0}\right)-m_{\varepsilon}\right)=0$;

(b) $\Gamma-\liminf _{\varepsilon \rightarrow 0}\left(F_{\varepsilon}(u)-m_{\varepsilon}\right)>0$ on $\overline{B_{\delta}\left(u_{0}\right)} \backslash\left\{u_{0}\right\}$.

Indeed condition (a) implies that we may change the constants $m_{\varepsilon}$ so that the $\Gamma$-limit exists, is 0 at $u_{0}$, and we have a recovery sequence with $F_{\varepsilon}\left(u_{\varepsilon}\right)=m_{\varepsilon}$, while (b) is kept unchanged. At this point is suffices to chose, e.g., $f(\varepsilon)=\varepsilon$.

Proof. The proof follows that of Theorem 4.1.1. Again, let $u_{\varepsilon}$ be a a minimizer of $F_{\varepsilon}$ on $\overline{B_{\delta}\left(u_{0}\right)}$; we can suppose that $u_{\varepsilon} \rightarrow \bar{u} \in \overline{B_{\delta}\left(u_{0}\right)}$ we then have

$$
\begin{aligned}
0=\widetilde{F}_{0}\left(u_{0}\right) & \leq \widetilde{F}_{0}(\bar{u}) \leq \liminf _{\varepsilon \rightarrow 0} \widetilde{F}_{\varepsilon}\left(u_{\varepsilon}\right)=\liminf _{\varepsilon \rightarrow 0} \frac{\min }{B_{\delta}\left(u_{0}\right)} \widetilde{F}_{\varepsilon} \\
& \leq \limsup _{\varepsilon \rightarrow 0} \frac{\inf }{B_{\delta}\left(u_{0}\right)} \widetilde{F}_{\varepsilon} \leq \inf _{B_{\delta}\left(u_{0}\right)} \widetilde{F}_{0}=0, .
\end{aligned}
$$

so that $\bar{u}=u_{0}$ and $u_{\varepsilon} \in B_{\delta}\left(u_{0}\right)$ for $\varepsilon$ small enough, which proves the thesis after remarking that (local) minimization of $F_{\varepsilon}$ and $\widetilde{F}_{\varepsilon}$ are equivalent up to additive and multiplicative constants.

Proposition 4.3.3 Let $F_{\varepsilon}$ satisfy the coerciveness and lower-semicontinuity assumptions of Theorem 4.1.1. Suppose furthermore that there exist a bounded positive function $f$ : $(0,+\infty) \rightarrow(0,+\infty)$, constants $m_{\varepsilon}$ and $\rho_{\varepsilon}$ with $\rho_{\varepsilon}>0$ and $\rho_{\varepsilon} \rightarrow 0$, and $\widetilde{u}_{\varepsilon} \rightarrow u_{0}$ such that the scaled functionals

$$
\widetilde{F}_{\varepsilon}(v)=\frac{F_{\varepsilon}\left(\widetilde{u}_{\varepsilon}+\rho_{\varepsilon} v\right)-m_{\varepsilon}}{f(\varepsilon)}
$$


are equicoercive and $\Gamma$-converge on $\overline{B_{\delta}\left(v_{0}\right)}$ to $\widetilde{F}_{0}$ with $v_{0}$ an isolated local minimum. Then there exists a sequence $\left(u_{\varepsilon}\right)$ converging to $u_{0}$ of local minimizers of $F_{\varepsilon}$.

Proof. We can apply Theorem 4.1.1 to the functionals $\widetilde{F}_{\varepsilon}(v)$ concluding that there exist local minimizers $v_{\varepsilon}$ of $\widetilde{F}_{\varepsilon}$ converging to $v_{0}$. The corresponding $u_{\varepsilon}=\widetilde{u}_{\varepsilon}+\rho_{\varepsilon} v_{\varepsilon}$ are local minimizers for $F_{\varepsilon}$ converging to $u_{0}$.

Example 4.3.4 We illustrate the proposition with the simple example

$$
F_{\varepsilon}(x)=\sin \left(\frac{x}{\varepsilon}\right)+x,
$$

whose $\Gamma$-limit $F(x)=x-1$ has no local (or global) minimizers. Take any $x_{0} \in \mathbb{R}, x_{\varepsilon} \rightarrow x_{0}$ any sequence with $\sin \left(x_{\varepsilon} / \varepsilon\right)=-1, m_{\varepsilon}=x_{\varepsilon}-1, \rho_{\varepsilon}=\varepsilon^{\beta}$ with $\beta \geq 1$, and $f(\varepsilon)=\varepsilon^{\alpha}$ with $\alpha \geq 0$, so that

$$
\begin{aligned}
\widetilde{F}_{\varepsilon}(t) & =\frac{\sin \left(\frac{x_{\varepsilon}+\varepsilon^{\beta} t}{\varepsilon}\right)+1}{\varepsilon^{\alpha}}+\varepsilon^{\beta-\alpha} t \\
& =\frac{\sin \left(\varepsilon^{\beta-1} t-\frac{\pi}{2}\right)+1}{\varepsilon^{\alpha}}+\varepsilon^{\beta-\alpha} t=\frac{1-\cos \left(\varepsilon^{\beta-1} t\right)}{\varepsilon^{\alpha}}+\varepsilon^{\beta-\alpha} t .
\end{aligned}
$$

In this case the $\Gamma$-limit $\widetilde{F}$ coincides with the pointwise limit of $\widetilde{F}_{\varepsilon}$. If $\beta=1$ and $0 \leq \alpha \leq 1$ then we have (local) minimizers of $\widetilde{F}$ at all points of $2 \pi \mathbb{Z}$; indeed if $\alpha=0$ then the sequence converges to $\widetilde{F}(x)=1-\cos x$, if $0<\alpha<1$ we have

$$
\widetilde{F}(x)= \begin{cases}0 & \text { if } x \in 2 \pi \mathbb{Z} \\ +\infty & \text { otherwise }\end{cases}
$$

and if $\alpha=1$

$$
\widetilde{F}(x)= \begin{cases}x & \text { if } x \in 2 \pi \mathbb{Z} \\ +\infty & \text { otherwise. }\end{cases}
$$

In the case $2>\beta>1$ we have two possibilities: if $\alpha=2 \beta-2$ then $\widetilde{F}(x)=\frac{1}{2} t^{2}$; if $\beta \geq \alpha>2 \beta-2$ then

$$
\widetilde{F}(x)= \begin{cases}0 & \text { if } x=0 \\ +\infty & \text { otherwise }\end{cases}
$$

If $\alpha=\beta=2$ then $\widetilde{F}(x)=\frac{1}{2} t^{2}+t$. In all these cases we have isolated local minimizers in the limit.

Note that in this computation $x_{\varepsilon}$ are not themselves local minimizers of $F_{\varepsilon}$.

We now consider an infinite-dimensional example in the same spirit as the one above. 
Example 4.3.5 (existence of infinitely many local minima for oscillating metrics) Let the 1 -periodic coefficient $a: \mathbb{R}^{2} \rightarrow\{1,2\}$ be defined on $[0,1]^{2}$ as

$$
a\left(v_{1}, v_{2}\right)= \begin{cases}1 & \text { if either }\left(v_{1}-v_{2}\right)\left(v_{1}+v_{2}-1\right)=0 \text { or } \\ 4 & \text { otherwise. }\end{cases}
$$

Let

$$
F_{\varepsilon}^{0}(u)=\int_{0}^{1} a\left(\frac{x}{\varepsilon}, \frac{u}{\varepsilon}\right)\left(1+\left|u^{\prime}\right|^{2}\right) d x
$$

defined on

$$
X=\left\{u \in W^{1, \infty}\left((0,1) ; \mathbb{R}^{2}\right), u(0)=0, u(1)=1\right\}
$$

equipped with the $L^{2}$-convergence. It may be useful to remark that $F_{\varepsilon}^{0}$ can be rewritten in terms of the curve $\gamma(x)=(x, u(x))$ as the energy

$$
\int_{0}^{1} a\left(\frac{\gamma}{\varepsilon}\right)\left|\gamma^{\prime}(x)\right|^{2} d x,
$$

of an inhomogeneous Riemannian metric which favors curves lying on the network where $a=1$ (we will call that the 1-network), which is a sort of opus reticolatum as pictured in Fig. 4.4.

The $\Gamma$-limit of $F_{\varepsilon}^{0}$ is of the form

$$
F_{\text {hom }}^{0}(u)=\int_{0}^{1} \varphi\left(u^{\prime}\right) d x .
$$

with domain $X$. It can be shown that $\varphi(z)=\sqrt{2}$ if $|z| \leq 1$, and that for functions with $\left|u^{\prime}\right| \leq 1$ recovery sequences for $F_{\text {hom }}^{0}(u)$ are functions with $a\left(x / \varepsilon, u_{\varepsilon}(x) / \varepsilon\right)=1$ a.e. (i.e., that follow the lines of the 1-network). This will also follow from the computations below.

We consider the functionals

$$
F_{\varepsilon}(u)=F_{\varepsilon}^{0}(u)+G(u), \quad \text { where } \quad G(u)=\int_{0}^{1}|u|^{2} d x
$$

(perturbation more general than $G$ can be added). Since $G$ is a continuous perturbation the $\Gamma$-limit of $F_{\varepsilon}$ is simply $F=F_{\text {hom }}^{0}+G$. Since $G$ is strictly convex, then $F$ is also strictly convex, and hence admits no local minimizers other than the absolute minimizer $u=0$. We will show that $F_{\varepsilon}$ admit infinitely many local minimizers. To that end we make some simplifying hypotheses: we suppose that $\varepsilon$ are of the form $2^{-k}$. In this way both $(0,0)$ and $(1,0)$ (corresponding to the boundary conditions) belong to the 1-network for all $\varepsilon$, and 1-networks are decreasing (in the sense of inclusion) with $\varepsilon$. We consider any function $u_{0} \in X$ such that $a\left(x 2^{k_{0}}, u_{0}(x) 2^{k_{0}}\right)=1$ a.e. for some $k_{0}$, and hence for all $k \geq k_{0}$; i.e., a function following the lines of the 1-network for all $\varepsilon$ sufficiently small. We will prove that every such $u_{0}$ is a local minimum for $F_{\varepsilon}$ if $\varepsilon$ is small enough. 
We consider the scaled functionals

$$
\widetilde{F}_{\varepsilon}(v)=\frac{F_{\varepsilon}\left(u_{0}+\varepsilon^{2} v\right)-F_{\varepsilon}\left(u_{0}\right)}{\varepsilon^{2}} .
$$

We note that the term deriving from $G$ still gives a continuously converging term, and can be dealt with separately, since

$$
\frac{G\left(u_{0}+\varepsilon^{2} v\right)-G\left(u_{0}\right)}{\varepsilon^{2}}=2 \int_{0}^{1} u_{0} v d x+\varepsilon^{2} \int_{0}^{1}|v|^{2} d x .
$$

We concentrate our analysis on the term of $\widetilde{F}_{\varepsilon}$ coming from $F_{\varepsilon}^{0}$ : let $\widetilde{v}_{\varepsilon}$ be such that

$$
\left\|\widetilde{v}_{\varepsilon}-u_{0}\right\|_{L^{2}} \leq \varepsilon^{2} \delta
$$

i.e., that $\widetilde{v}_{\varepsilon}=u_{0}+\varepsilon^{2} v_{\varepsilon}$ with $\left\|v_{\varepsilon}\right\|_{L^{2}} \leq \delta$, and $\widetilde{F}_{\varepsilon}\left(\widetilde{v}_{\varepsilon}\right) \leq C_{1}<+\infty$.

We denote $\widetilde{\gamma}_{\varepsilon}(x)=\left(x, \widetilde{v}_{\varepsilon}(x)\right)$ and $\gamma_{0}(x)=\left(x, u_{0}(x)\right)$. Note that if we set $e^{1}=\left(\frac{1}{\sqrt{2}}, \frac{1}{\sqrt{2}}\right)$ and $e^{2}=\left(\frac{1}{\sqrt{2}},-\frac{1}{\sqrt{2}}\right)$ then $x \mapsto\left\langle\gamma_{0}(x), e^{1}\right\rangle$ and $x \mapsto\left\langle\gamma_{0}(x), e^{2}\right\rangle$ are both non decreasing. We may then suppose that the same holds for $\widetilde{\gamma}_{\varepsilon}$. We also denote

$$
\|z\|_{1}=\left|\left\langle z, e^{1}\right\rangle\right|+\left|\left\langle z, e^{2}\right\rangle\right|
$$

For each $\varepsilon$ fixed we consider points $0=x_{0}<x_{1}<\ldots<x_{N}=1$ such that

$$
a\left(\frac{\widetilde{\gamma}_{\varepsilon}}{\varepsilon}\right)=1 \quad \text { a.e. or } \quad a\left(\frac{\widetilde{\gamma}_{\varepsilon}}{\varepsilon}\right)=4 \quad \text { a.e. alternately on }\left[x_{k-1}, x_{k}\right] ;
$$

we can suppose that the first case occurs for e.g. $k$ odd and the second one for $k$ even. In the first case, by convexity and taking into account that the image of $\widetilde{\gamma}_{\varepsilon}$ is contained in the 1-network, we have

$$
\int_{x_{k-1}}^{x_{k}} a\left(\frac{\widetilde{\gamma}_{\varepsilon}}{\varepsilon}\right)\left|\widetilde{\gamma}_{\varepsilon}\right|^{2} d x \geq\left(x_{k}-x_{k-1}\right)\left\|\frac{\widetilde{\gamma}_{\varepsilon}\left(x_{k}\right)-\widetilde{\gamma}_{\varepsilon}\left(x_{k-1}\right)}{x_{k}-x_{k-1}}\right\|_{1}^{2}
$$

In the second case, again by convexity and by the inequality $\|z\|_{1} \leq \sqrt{2}|z|$,

$$
\begin{aligned}
\int_{x_{k-1}}^{x_{k}} a\left(\frac{\widetilde{\gamma}_{\varepsilon}}{\varepsilon}\right)\left|\widetilde{\gamma}_{\varepsilon}^{\prime}\right|^{2} d x & \geq 4\left(x_{k}-x_{k-1}\right)\left|\frac{\widetilde{\gamma}_{\varepsilon}\left(x_{k}\right)-\widetilde{\gamma}_{\varepsilon}\left(x_{k-1}\right)}{x_{k}-x_{k-1}}\right|^{2} \\
& \geq 2\left(x_{k}-x_{k-1}\right)\left\|\frac{\widetilde{\gamma}_{\varepsilon}\left(x_{k}\right)-\widetilde{\gamma}_{\varepsilon}\left(x_{k-1}\right)}{x_{k}-x_{k-1}}\right\|_{1}^{2}
\end{aligned}
$$

As a first consequence, we deduce that

$$
F_{\varepsilon}^{0}\left(\widetilde{\gamma}_{\varepsilon}\right) \geq \sum_{k=1}^{N}\left(x_{k}-x_{k-1}\right)\left\|\frac{\widetilde{\gamma}_{\varepsilon}\left(x_{k}\right)-\widetilde{\gamma}_{\varepsilon}\left(x_{k-1}\right)}{x_{k}-x_{k-1}}\right\|_{1}^{2}+\sum_{k \text { even }}\left(x_{k}-x_{k-1}\right)\left\|\frac{\widetilde{\gamma}_{\varepsilon}\left(x_{k}\right)-\widetilde{\gamma}_{\varepsilon}\left(x_{k-1}\right)}{x_{k}-x_{k-1}}\right\|_{1}^{2}
$$




$$
\begin{aligned}
& \geq\left\|\sum_{k=1}^{N} \widetilde{\gamma}_{\varepsilon}\left(x_{k}\right)-\widetilde{\gamma}_{\varepsilon}\left(x_{k-1}\right)\right\|_{1}^{2}+\sum_{k \text { even }} \frac{1}{x_{k}-x_{k-1}}\left\|\widetilde{\gamma}_{\varepsilon}\left(x_{k}\right)-\widetilde{\gamma}_{\varepsilon}\left(x_{k-1}\right)\right\|_{1}^{2} \\
& =\left\|\widetilde{\gamma}_{\varepsilon}(1)-\widetilde{\gamma}_{\varepsilon}(0)\right\|_{1}^{2}+\sum_{k \text { even }} \frac{1}{x_{k}-x_{k-1}}\left\|\widetilde{\gamma}_{\varepsilon}\left(x_{k}\right)-\widetilde{\gamma}_{\varepsilon}\left(x_{k-1}\right)\right\|_{1}^{2} \\
& =F_{\varepsilon}^{0}\left(u_{0}\right)+\sum_{k \text { even }} \frac{1}{x_{k}-x_{k-1}}\left\|\widetilde{\gamma}_{\varepsilon}\left(x_{k}\right)-\widetilde{\gamma}_{\varepsilon}\left(x_{k-1}\right)\right\|_{1}^{2} .
\end{aligned}
$$

From the energy bound we then deduce that for each $k$ even

$$
\left(x_{k}-x_{k-1}\right)\left(1+\left(\frac{\widetilde{v}_{\varepsilon}\left(x_{k}\right)-\widetilde{v}_{\varepsilon}\left(x_{k-1}\right)}{x_{k}-x_{k-1}}\right)\right) \leq C \varepsilon^{2} .
$$

so that both $\left(x_{k}-x_{k-1}\right) \leq C \varepsilon^{2}$ and $\left|\widetilde{v}_{\varepsilon}\left(x_{k}\right)-\widetilde{v}_{\varepsilon}\left(x_{k-1}\right)\right| \leq C \varepsilon$. This implies that $\widetilde{\gamma}_{\varepsilon}$ can be deformed with a perturbation with $o\left(\varepsilon^{2}\right) L^{2}$-norm to follow the 1-network between $x_{k-1}$ and $x_{k}$. Hence, possible competitors essentially follow the 1-network (see Fig. 4.4). If $\delta$ is

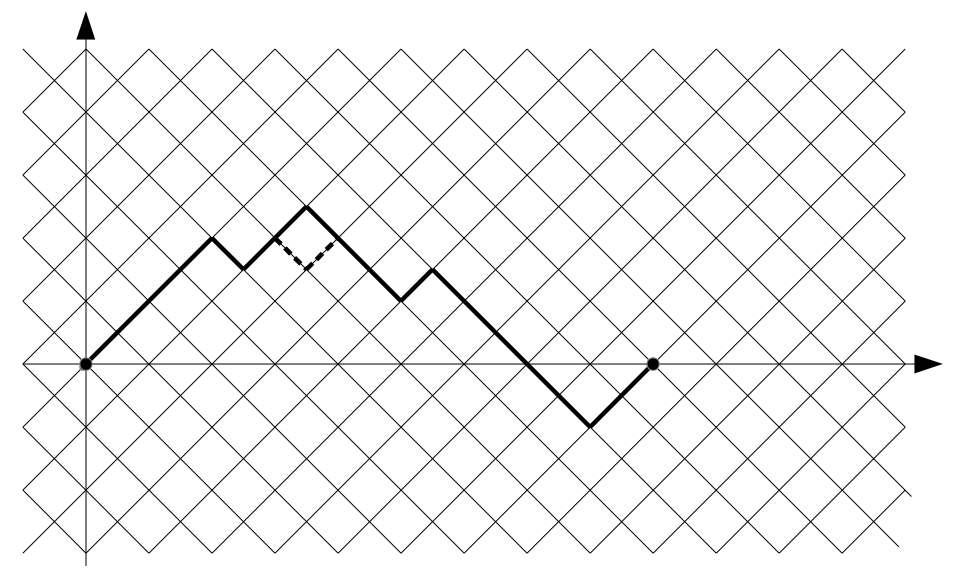

Figure 4.4: a local minimizer and a competitor

small enough then in order that (4.8) hold we must have $v_{\varepsilon} \rightarrow 0$. This shows that the limit of $\widetilde{F}_{\varepsilon}$ is finite only at $v=0$ on $B_{\delta}(0)$ as desired.

As a consequence of the computation above we deduce that for all $u \in X$ with $\left\|u^{\prime}\right\| \infty \leq$ 1 we have a sequence $\left\{u_{\varepsilon}\right\}$ of local minimizers of $F_{\varepsilon}$ converging to $u$.

Example 4.3.6 (density of local minima for oscillating distances) We may consider a similar example to the one above for oscillating distances; i.e., length functionals defined 
on curves. Let the 1-periodic coefficient $a: \mathbb{R}^{2} \rightarrow\{1,2\}$ be defined as

$$
a\left(v_{1}, v_{2}\right)= \begin{cases}1 & \text { if either } v_{1} \text { or } v_{2} \in \mathbb{Z} \\ 4 & \text { otherwise. }\end{cases}
$$

This is the same type of coefficient as in the previous example up to a rotation and a scaling factor. Let

$$
F_{\varepsilon}(u)=\int_{0}^{1} a\left(\frac{u}{\varepsilon}\right)\left|u^{\prime}\right| d x
$$

be defined on

$$
X=\left\{u \in W^{1,1}\left((0,1) ; \mathbb{R}^{2}\right), u(0)=v_{0}, u(1)=v_{1}\right\}
$$

equipped with the $L^{1}$-convergence.

The $\Gamma$-limit of $F_{\varepsilon}$ is

$$
F(u)=\int_{0}^{1}\left\|u^{\prime}\right\|_{1} d x,
$$

where

$$
\|z\|_{1}=\left|z_{1}\right|+\left|z_{2}\right|
$$

This is easily checked after remarking that recovery sequences $\left(u_{\varepsilon}\right)$ are such that $a\left(u_{\varepsilon}(t) / \varepsilon\right)=$ 1 a.e. (except possibly close to 0 and 1 if $a\left(v_{0} / \varepsilon\right) \neq 1$ or $a\left(v_{1} / \varepsilon\right) \neq 1$ ) and then that $\left|u_{\varepsilon}^{\prime}\right|=\left|\left(u_{\varepsilon}\right)_{1}^{\prime}\right|+\left|\left(u_{\varepsilon}\right)_{2}^{\prime}\right|$. For example, if both components of $\left(u_{\varepsilon}\right)$ are monotone, then

$$
\begin{aligned}
F_{\varepsilon}\left(u_{\varepsilon}\right) & =\int_{0}^{1} a\left(\frac{u_{\varepsilon}}{\varepsilon}\right)\left|u^{\prime}\right| d x=\int_{0}^{1}\left|u_{\varepsilon}^{\prime}\right| d x+o(1) \\
& =\int_{0}^{1}\left(\left|\left(u_{\varepsilon}\right)_{1}^{\prime}\right|+\left|\left(u_{\varepsilon}\right)_{2}^{\prime}\right|\right) d x+o(1) \\
& =\left|\left(v_{1}\right)_{1}-\left(v_{0}\right)_{1}\right|+\left|\left(v_{1}\right)_{2}-\left(v_{0}\right)_{2}\right|+o(1) \\
& =\int_{0}^{1}\left(\left|u_{1}^{\prime}\right|+\left|u_{2}^{\prime}\right|\right) d x+o(1)=F(u)+o(1) .
\end{aligned}
$$

For all these energies there are no strict local minimizers since energies are invariant with respect to reparameterization. Anyhow, if we consider equivalence classes with respect to reparameterization (e.g., by taking only functions in

$$
\left.X^{1}=\left\{u \in X:\left\|u^{\prime}\right\|_{1} \text { constant a.e. }\right\}\right)
$$

then an argument similar to the one in the previous example shows that local minimizers are $L^{1}$ dense, in the sense that for all $u \in X^{1}$ there exists a sequence of local minimizers of $F_{\varepsilon}$ (restricted to $X^{1}$ ) converging to $u$.

As a technical remark, we note that in order to have coercivity the limit $F$ should be extended to the space of curves with bounded variations. Anyhow, since functionals are invariant by reparameterization, it suffices to consider bounded sequences in $W^{1, \infty}$ after a change of variables. 


\subsection{References to Chapter 4}

The use of Theorem 4.1.1 for proving the existence of local minimizers, together with Example 4.2.2 are due to Kohn and Sternberg [30]. 


\section{Chapter 5}

\section{Small-scale stability}

The notion of local minimizer is 'scale-independent'; i.e., it does not depend on the rate at which energies converge, so that it does not discriminate, e.g., between energies

$$
F_{\varepsilon}(x)=x^{2}+\sin ^{2}\left(\frac{x}{\varepsilon}\right) \quad \text { or } \quad F_{\varepsilon}(x)=x^{2}+\sqrt{\varepsilon} \sin ^{2}\left(\frac{x}{\varepsilon}\right) .
$$

We now examine a notion of stability such that, loosely speaking, a point is stable if it is not possible to reach a lower energy state from that point without crossing an energy barrier of a specified height. In this case the local minimizers in the first of the two sequence of energies are stable as $\varepsilon \rightarrow 0$, while those in the second sequence are not.

\subsection{Larsen's stable points}

We first introduce a notion of stability that often can be related to notions of local minimality.

Definition 5.1.1 (slide) Let $F: X \rightarrow[0,+\infty]$ and $\delta>0$. A continuous function $\phi$ : $[0,1] \rightarrow X$ is a $\delta$-slide for $F$ at $u_{0} \in X$ if

- $\phi(0)=u_{0}$ and $F(\phi(1))<F(\phi(0))=F\left(u_{0}\right)$;

- there exits $\delta^{\prime}<\delta$ such that $F(\phi(t)) \leq F(\phi(s))+\delta^{\prime}$ if $0 \leq s \leq t \leq 1$.

Definition 5.1.2 (stability) Let $F: X \rightarrow[0,+\infty]$ and $\delta>0$. A point $u_{0} \in X$ is $\delta$-stable for $F$ if no $\delta$-slide exists for $F$ at $u_{0}$.

A point $u_{0} \in X$ is stable for $F$ if it is $\delta$-stable for some $\delta>0$ (and hence for all $\delta$ small enough).

Let $F_{\varepsilon}: X \rightarrow[0,+\infty]$. A sequence of points $\left(u_{\varepsilon}\right)$ in $X$ is uniformly stable for $\left(F_{\varepsilon}\right)$ if there exists $\delta>0$ such that all $u_{\varepsilon}$ are $\delta$-stable for $\varepsilon$ small. 
Example 5.1.3 (1) $F(x)=\left\{\begin{array}{ll}0 & x=0 \\ \sin \left(\frac{1}{x}\right) & \text { otherwise. }\end{array}\right.$ The point 0 is not a local minimizer but it is $\delta$-stable for $\delta<1$;

(2) Similarly for $F(x)= \begin{cases}0 & x=0 \\ -x^{2}+\sin ^{2}\left(\frac{1}{x}\right) & \text { otherwise; }\end{cases}$

(3) Let $X=\mathbb{C}$ and $F(z)=F\left(\rho e^{i \theta}\right)= \begin{cases}\theta \rho & |z| \leq 1 \\ -1 & \text { otherwise, }\end{cases}$

where we have chosen the representation $z=\rho e^{i \theta}$ with $0<\theta \leq 2 \pi$. Then 0 is an isolated local minimum, but it is not stable; e.g., taking $\phi(t)=2 t e^{i \delta / 2}$. Note in fact that $\phi(0)=0$, $F(\phi(1))=-1<0$, and $\sup F(\phi(t))=F(\phi(1 / 2))=\delta / 2$;

(4) We can generalize example (3) to an infinite-dimensional example. Take $X=$ $L^{2}(-\pi, \pi)$ and

$$
F(u)= \begin{cases}\sum_{k} \frac{1}{k^{2}}\left|c_{k}\right|^{2} & \text { if } u=\sum_{k} c_{k} e^{i k x} \text { and }\|u\|_{L^{2}}<1 \\ -1 & \text { otherwise. }\end{cases}
$$

The constant 0 is an isolated minimum point. $F$ is lower semicontinuous, and continuous in $\left\{\|u\|_{L^{2}}<1\right\}$. Note that $F\left(e^{i k x}\right)=\frac{1}{k^{2}}$ so that $\phi_{k}(t)=2 t e^{i k x}$ is a $\delta$-slide for $k^{2}>1 / \delta$; stable;

(5) $F_{\varepsilon}(x)=x^{2}+\sin ^{2}\left(\frac{x}{\varepsilon}\right)$. Each bounded sequence of local minimizers is uniformly

(6) $F_{\varepsilon}(x)=x^{2}+\varepsilon^{\alpha} \sin ^{2}\left(\frac{x}{\varepsilon}\right)$ with $0<\alpha<1$. No bounded sequence of local minimizers is uniformly stable (except the constant sequence of global minimizers $x_{\varepsilon}=0$ ).

Remark 5.1.4 (local minimality and stability) (i) If $F: X \rightarrow \mathbb{R}$ is continuous and $u$ stable; then $u$ is a local minimizer;

(ii) Let $F$ be lower semicontinuous and coercive. Then every isolated local minimizer of $F$ is stable.

(iii) if $u$ is just a local minimizer then $u$ may not be stable.

To check (i) suppose that $u$ is not a local minimum for $F$. Then let $\rho$ be such that $|F(u)-F(w)|<\delta / 2$ if $w \in B_{\rho}(u)$, and let $u_{\rho} \in B_{\rho}(u)$ be such that $F\left(u_{\rho}\right)<F(u)$. Then it suffices to take $\phi(t)=u+t\left(u_{\rho}-u\right)$.

To check (ii), let $\eta>0$ be such that $u_{0}$ is an isolated minimum point in $B_{\eta}\left(u_{0}\right)$. If $u_{0}$ is not stable then there exist $1 / k$ slides $\phi_{k}$ with final point outside $B_{\eta}\left(u_{0}\right)$. This implies that there exist $u_{k}=\phi_{k}\left(t_{k}\right)$ for some $t_{k}$ with $u_{k} \in \partial B_{\eta}\left(u_{0}\right)$, so that $F\left(u_{k}\right) \leq F\left(u_{0}\right)+1 / k$. By coerciveness, upon extraction of a subsequence $u_{\varepsilon} \rightarrow \bar{u} \in \partial B_{\eta}\left(u_{0}\right)$, and by lower semicontinuity $F(\bar{u}) \leq \liminf _{k} F\left(u_{k}\right) \leq F\left(u_{0}\right)$, which is a contradiction.

For (iii) take for example $u=0$ for $F(u)=(1-|u|) \wedge 0$ on $\mathbb{R}$. 


\subsection{Stable sequences of functionals}

We now give a notion of stability of parameterized functionals.

Definition 5.2.1 (relative (sub)stability) We say that a sequence $\left(F_{\varepsilon}\right)$ is (sub)stable relative to $F$ if the following holds

- if $u_{0}$ has a $\delta$-slide for $F$ and $u_{\varepsilon} \rightarrow u_{0}$, then each $u_{\varepsilon}$ has a $\delta$-slide for $F_{\varepsilon}$ (for $\varepsilon$ small enough).

Remark 5.2.2 (relative (super)stability) The condition of sub-stability above can be compared to the lower bound for $\Gamma$-convergence. With this parallel in mind we can introduce a notion of (super) stability relative to $F$ by requiring that

- if $u_{0}$ is an isolated local minimum for $F$ then there exists $u_{\varepsilon} \rightarrow u$ such that $\left(u_{\varepsilon}\right)$ is uniformly stable for $F_{\varepsilon}$.

Remark 5.2.3 (i) Note that if $F$ is a constant then all $\left(F_{\varepsilon}\right)$ are stable relative to $F$;

(ii) In general if $F_{\varepsilon}=F$ for all $\varepsilon$ then $\left(F_{\varepsilon}\right)$ may not be stable relative to $F$. Take for example

$$
F_{\varepsilon}(x)=F(x)= \begin{cases}\sin \left(\frac{1}{x}\right) & \text { if } x>0 \\ x & \text { if } x \leq 0\end{cases}
$$

then $x_{0}=0$ has $\delta$-slides for all $\delta>0$, while taking $x_{\varepsilon}=\left(2 \pi\left\lfloor\frac{1}{\varepsilon}\right\rfloor-\frac{\pi}{2}\right)^{-1}$ we have $x_{\varepsilon} \rightarrow x_{0}$ and $x_{\varepsilon}$ has no $\delta$-slide for $\delta<2$.

The following proposition is in a sense the converse of Theorem 4.1.1 with $\Gamma$-convergence substituted with stability.

Proposition 5.2.4 Let $\left(F_{\varepsilon}\right)$ be (sub)stable relative to $F$ and $u_{\varepsilon}$ be a sequence of uniformly stable points for $F_{\varepsilon}$ with $u_{\varepsilon} \rightarrow u$. Then $u$ is stable for $F$.

Proof. If $u_{\varepsilon} \rightarrow u$ and $u_{\varepsilon}$ is uniformly stable then it is stable for some $\delta>0$. By the (sub)stability of $\left(F_{\varepsilon}\right)$ then $u$ is $\delta^{\prime}$ stable for all $0<\delta^{\prime}<\delta$; i.e., it is stable.

Remark 5.2.5 The main drawback of the notion of stability of energies is that it is not in general compatible with the addition of (continuous) perturbations. Take for example $F_{\varepsilon}(x)=\sin ^{2}\left(\frac{x}{\varepsilon}\right)$ and $F=0$. Then $F_{\varepsilon}$ is stable relative to $F$, but $G_{\varepsilon}(x)=F_{\varepsilon}(x)+x$ is not stable with respect to $G(x)=x$ : each $x$ has a $\delta$-slide for all $\delta>0$, but if $x_{\varepsilon} \rightarrow x$ is a sequence of local minimizers of $G_{\varepsilon}$ then they are $\delta$-stable for $\delta<1$. 


\subsection{Stability and $\Gamma$-convergence}

In this section we will couple stability with $\Gamma$-convergence, and try to derive some criteria in order to guarantee the compatibility with respect to the addition of continuous perturbations. The main issue is to construct $\delta$-slides for the approximating functionals starting from $\delta$-slides for the $\Gamma$-limit.

Example 5.3.1 We consider the one-dimensional energies

$$
F_{\varepsilon}(u)=\int_{0}^{1} a\left(\frac{x}{\varepsilon}\right)\left|u^{\prime}\right|^{2} d x
$$

where $a$ is a 1 -periodic function with $0<\inf a<\sup a<+\infty$, so that $F_{\varepsilon} \Gamma$-converge to the Dirichlet integral

$$
F(u)=\underline{a} \int_{0}^{1}\left|u^{\prime}\right|^{2} d x .
$$

We will also consider a perturbation of $F_{\varepsilon}$ with

$$
G(u)=\int_{0}^{1} g(x, u) d x
$$

where $g$ is a Carathéodory function with $|g(x, u)| \leq C\left(1+|u|^{2}\right)$ (this guarantees that $G$ is $L^{2}$-continuous).

We want to check that $F_{\varepsilon}+G$ is stable relative to $F+G$. To this end consider a point $u_{0}$ such that a $\delta$-slide $\phi$ for $F+G$ exists at $u_{0}$, and points $u_{\varepsilon} \rightarrow u_{0}$. We wish to construct a $\delta$-slide for $F_{\varepsilon}+G$ at $u_{\varepsilon}$.

With fixed $K \in \mathbb{N}$ we consider points $x_{i}^{K}=i / K$ for $i=0, \ldots K$ and denote for every $t$ with $\phi^{K}(t)$ the piecewise affine interpolation of $\phi(t)$ on the points $x_{i}^{K}$. Note that we have

- for all $K$ we have $F\left(\phi^{K}(t)\right) \leq F(\phi(t))$ by Jensen's inequality;

- $F\left(\phi^{K}(t)\right) \rightarrow F(\phi(t))$ as $K \rightarrow+\infty$;

- for fixed $K$ the map $t \mapsto \phi^{K}(t)$ is continuous with respect to the strong $H^{1}$-convergence. Indeed its gradient is piecewise constant and is weakly continuous in $t$, hence it is strongly continuous.

We fix $\delta^{\prime}<\delta$ such that

$$
F(\phi(t))+G(\phi(t)) \leq F(\phi(s))+G(\phi(s))+\delta^{\prime} \text { if } 0 \leq s \leq t \leq 1,
$$

choose $\delta^{\prime \prime}>0$ such that $\delta^{\prime}+2 \delta^{\prime \prime}<\delta$ and

$$
F(\phi(1))+G(\phi(1))<F\left(u_{0}\right)+G\left(u_{0}\right)-2 \delta^{\prime} .
$$

Let $K$ be large enough so that (if $u_{0}^{K}=\phi^{K}(0)$ denotes the interpolation of $u_{0}$ )

$$
F\left(u_{0}^{K}\right)+G\left(u_{0}^{K}\right) \geq F\left(u_{0}\right)+G\left(u_{0}\right)-\delta^{\prime \prime}
$$


and

$$
\left|G\left(\phi^{K}(t)\right)-G(\phi(t))\right|<\delta^{\prime \prime}
$$

for all $t$. We then have

$$
F\left(\phi^{K}(t)\right)+G\left(\phi^{K}(t)\right) \leq F(\phi(t))+G(\phi(t))+\delta^{\prime \prime} .
$$

We then claim that, up to a reparameterization, $\phi^{K}$ is a $\delta$-slide for $F+G$ from $u_{0}^{K}$.

Indeed, let $M=\inf \left\{t: F\left(\phi^{K}(t)\right)+G\left(\phi^{K}(t)\right)<F\left(u_{0}^{K}\right)+G\left(u_{0}^{K}\right)\right\}$. This set is not empty since it contains the point 1 . If $0 \leq s \leq t \leq M$ then we have

$$
\begin{aligned}
& F\left(\phi^{K}(t)\right)+G\left(\phi^{K}(t)\right)-F\left(\phi^{K}(s)\right)+G\left(\phi^{K}(s)\right) \\
\leq & \sup \left\{F\left(\phi^{K}(r)\right)+G\left(\phi^{K}(r)\right): 0 \leq r \leq M\right\}-F\left(u_{0}^{K}\right)+G\left(u_{0}^{K}\right) \\
\leq & \sup \{F(\phi(r))+G(\phi(r)): 0 \leq r \leq M\}+2 \delta^{\prime \prime}-F\left(u_{0}\right)+G\left(u_{0}\right) \\
\leq & \delta^{\prime}+2 \delta^{\prime \prime}<\delta
\end{aligned}
$$

By the continuity of $t \mapsto F\left(\phi^{K}(t)\right)+G\left(\phi^{K}(t)\right)$ we can then find $\bar{t}>M$ such that $F\left(\phi^{K}(\bar{t})\right)+$ $G\left(\phi^{K}(\bar{t})\right)<F\left(u_{0}^{K}\right)+G\left(u_{0}^{K}\right)$ and $s \mapsto \Phi^{K}(s \bar{t})$ is a $\delta$-slide. For the following, we suppose that $\bar{t}=1$, so that we do not need any reparameterization.

Next, we construct a $\delta$-slide for $F_{\varepsilon}+G$. To this end, for the sake of simplicity, we assume that $N=\frac{1}{\varepsilon K} \in \mathbb{N}$. Let $v$ be a function in $H_{0}^{1}(0,1)$ such that

$$
\int_{0}^{1} a(y)\left|v^{\prime}+1\right|^{2} d y=\min \left\{\int_{0}^{1} a(y)\left|w^{\prime}+1\right|^{2} d y: w \in H_{0}^{1}(0,1)\right\}=\underline{a} .
$$

Note that we also have

$$
\int_{0}^{N} a(y)\left|v^{\prime}+1\right|^{2} d y=\min \left\{\int_{0}^{N} a(y)\left|w^{\prime}+1\right|^{2} d y: w \in H_{0}^{1}(0,1)\right\}=N \underline{a} .
$$

We then define the function $\phi_{\varepsilon}^{K}(t)$ by setting on $\left[x_{i}^{K}, x_{i+1}^{K}\right]$

$$
\phi_{\varepsilon}^{K}(t)\left(x_{i}^{K}+s\right)=\phi(t)\left(x_{i}^{K}\right)+K\left(\phi(t)\left(x_{i+1}^{K}\right)-\phi(t)\left(x_{i}^{K}\right)\right)\left(s+\varepsilon v\left(\frac{s}{\varepsilon}\right)\right), \quad 0 \leq s \leq \frac{1}{K},
$$

so that

$$
F_{\varepsilon}\left(\phi_{\varepsilon}^{K}(t)\right)=F\left(\phi^{K}(t)\right) .
$$

Note again that we may suppose $\varepsilon$ small enough so that $\left|G\left(\phi_{\varepsilon}^{K}(t)\right)-G\left(\phi^{K}(t)\right)\right|=o(1)$ uniformly in $t$ so that $\phi_{\varepsilon}^{K}$ is a $\delta$-slide for $F_{\varepsilon}+G$ at $\phi_{\varepsilon}^{K}(0)$.

It now remains to construct a $L^{2}$-continuous function $\psi_{\varepsilon}:[0,1] \rightarrow H^{1}(0,1)$ with $\psi_{\varepsilon}(0)=u_{\varepsilon}$ and $\psi_{\varepsilon}(1)=\phi_{\varepsilon}^{K}(0)$ such that concatenating $\psi_{\varepsilon}$ with $\phi_{\varepsilon}^{K}$ we have a $\delta$-slide. This is achieved by taking the affine interpolation (in $t$ ) of $u_{\varepsilon}$ and $u_{\varepsilon}^{K}$ defined by setting on $\left[x_{i}^{K}, x_{i+1}^{K}\right]$

$$
u_{\varepsilon}^{K}\left(x_{i}^{K}+s\right)=u_{\varepsilon}\left(x_{i}^{K}\right)+K\left(u_{\varepsilon}\left(x_{i+1}^{K}\right)-u_{\varepsilon}\left(x_{i}^{K}\right)\right)\left(s+\varepsilon v\left(\frac{s}{\varepsilon}\right)\right), \quad 0 \leq s \leq \frac{1}{K},
$$

on $(0,1 / 2)$ and of $u_{\varepsilon}^{K}$ and $\phi_{\varepsilon}^{K}(0)$ on $(1 / 2,1)$. 
Example 5.3.2 We consider the oscillating perimeter functionals $F_{\varepsilon}$ and $F$ of Example 3.5.1 We now show that if $A$ has a $\delta$-slide for $F$ and $A_{\varepsilon} \rightarrow A$, then each $A_{\varepsilon}$ has a $(\delta+o(1))$ slide for $F_{\varepsilon}$ (and so a $\delta$-slide for $\varepsilon$ sufficiently small). If is easily checked that the same argument can be used if we add to $F_{\varepsilon}$ a continuous perturbation

$$
G(A)=\int_{A} f(x) d x
$$

where $f$ is a (smooth) bounded function, so that the stability can be used also for $F_{\varepsilon}+G$.

We first observe that an arbitrary sequence $A_{\varepsilon}$ of Lipschitz sets converging to a set $A$ can be substituted by a sequence in $\mathcal{A}_{\varepsilon}$ with the same limit. To check this, consider a connected component of $\partial A_{\varepsilon}$. Note that for $\varepsilon$ small enough every portion of $\partial A_{\varepsilon}$ parameterized by a curve $\gamma:[0,1] \rightarrow \mathbb{R}^{2}$ such that $a(\gamma(0) / \varepsilon)=a(\gamma(1) / \varepsilon)=1$ and $a(\gamma(t) / \varepsilon)=2$ for $0<t<1$ can be deformed continuously to a curve lying on $\varepsilon a^{-1}(1)$ and with the same endpoints. If otherwise a portion of $\partial A_{\varepsilon}$ lies completely inside a cube $Q_{i}^{\varepsilon}$ it can be shrunk to a point or expanded to the whole cube $Q_{i}^{\varepsilon}$. In both cases this process can be obtained by a $O(\varepsilon)$ slide, since either the lengths of the curves are bounded by $2 \varepsilon$, or the deformation can be performed so that the lengths are decreasing.

We can therefore assume that $A_{\varepsilon} \in \mathcal{A}_{\varepsilon}$ and that there exist a $\delta$-slide for $E$ at $A$ obtained by a continuous family $A(t)$ with $0 \leq t \leq 1$.

We fix $N \in \mathbb{N}$ and set $t_{j}^{N}=j / N$. For all $j \in\{1, \ldots, N\}$ let $A_{\varepsilon}^{N, j}$ be a recovery sequence in $\mathcal{A}_{\varepsilon}$ for $A\left(t_{j}^{N}\right)$. Furthermore we set $A_{n}^{N, 0}=A_{\varepsilon}$. Note that, since $A_{\varepsilon}^{N, j} \rightarrow A\left(t_{j}^{N}\right)$ and $A(t)$ is continuous, we have $\left|A_{\varepsilon}^{N, j} \triangle A_{\varepsilon}^{N, j+1}\right|=o(1)$ as $N \rightarrow+\infty$. We may suppose that the set $A_{\varepsilon}^{N, j+1}$ is the union of $A_{\varepsilon}^{N, j}$ and a family of cubes $Q_{i}^{N, j}$. We may order the indices $i$ and construct a continuous family of sets $A^{N, j, i}(t)$ such that $A^{N, j, i}(0)=A_{\varepsilon}^{N, j} \cup \bigcup_{k<i} Q_{k}^{N, j}$, $A^{N, j, i}(1)=A_{\varepsilon}^{N, j} \cup \bigcup_{k \leq i} Q_{k}^{N, j}$,

$$
\left(\mathcal{H}^{1}\left(A_{\varepsilon}^{N, j}\right) \wedge \mathcal{H}^{1}\left(A_{\varepsilon}^{N, j+1}\right)\right)-C \varepsilon \leq \mathcal{H}^{1}\left(A^{N, j, i}(t)\right) \leq\left(\mathcal{H}^{1}\left(A_{\varepsilon}^{N, j}\right) \vee \mathcal{H}^{1}\left(A_{\varepsilon}^{N, j+1}\right)\right)+C \varepsilon .
$$

Since also $\left|A^{N, j, i}(t)\right|$ differs from $\left|A_{\varepsilon}^{N, j}\right|$ and $\left|A_{\varepsilon}^{N, j+1}\right|$ by at most $o(1)$ as $N \rightarrow+\infty$, by concatenating all these families, upon reparametrization we obtain a family $A_{n}^{N}(t)$ such that $A_{n}^{N}(0)=A_{\varepsilon}, A_{n}^{N}(1)=A_{\varepsilon}(1)$, and, if $s<t$ then we have, for some $j<k$

$$
\begin{aligned}
& F_{\varepsilon}\left(A_{n}^{N}(s)\right) \geq F\left(A\left(t_{j}^{N}\right)\right)-C \varepsilon-o(1), \\
& F_{\varepsilon}\left(A_{n}^{N}(t)\right) \leq F\left(A\left(t_{k}^{N}\right)\right)+C \varepsilon+o(1) .
\end{aligned}
$$

Since $A(t)$ is a $\delta$-slide for $E$ we have

$$
F\left(A\left(t_{k}^{N}\right)\right) \leq F\left(A\left(t_{j}^{N}\right)\right)+\varepsilon
$$

so that

$$
F_{\varepsilon}\left(A_{n}^{N}(t)\right) \leq F_{\varepsilon}\left(A_{n}^{N}(s)\right)+\delta+C \varepsilon+o(1)
$$

By choosing $N$ large enough and $\varepsilon$ small enough we obtain the desired $(\delta+o(1))$-slide. 
The previous example suggests a criterion for 'strong' stability (i.e., compatible with continuous perturbations), which is sometimes satisfied by $\Gamma$-converging sequences. We have constructed $\delta$-slides for the approximating functionals in two steps: one in which we have transformed a limit $\delta$-slide $\phi(t)$ considering recovery sequences (essentially, setting $\phi_{\varepsilon}(t)=u_{\varepsilon}^{t}$, where $\left(u_{\varepsilon}^{t}\right)$ is a recovery sequence for $\left.\phi(t)\right)$, another where we have constructed an 'almost-decreasing' path from $u_{\varepsilon}$ to $\phi_{\varepsilon}(0)$. Note that this step, conversely, is possible thanks to the liminf inequality.

Theorem 5.3.3 (a criterion of strong stability) Suppose that $F_{\varepsilon}$ and $F$ satisfy the following requirements:

if $\phi$ is a path from $u$ (i.e., $\phi:[0,1] \rightarrow X, \phi(0)=u$, and $\phi$ is continuous) and $u_{\varepsilon} \rightarrow u$, then there exist paths $\psi_{\varepsilon}$ from $u_{\varepsilon}$ and $\phi_{\varepsilon}$ from $\psi_{\varepsilon}(1)$ such that

(i) $\tau \mapsto F_{\varepsilon}\left(\psi_{\varepsilon}(\tau)\right)$ is decreasing up to o(1) as $n \rightarrow+\infty$; i.e.,

$$
\sup _{0 \leq \tau_{1}<\tau_{2} \leq 1}\left(F_{\varepsilon}\left(\psi_{\varepsilon}\left(\tau_{2}\right)\right)-F_{\varepsilon}\left(\psi_{\varepsilon}\left(\tau_{1}\right)\right)\right) \rightarrow 0 \text { as } n \rightarrow \infty
$$

(ii) $\sup _{\tau \in[0,1]} \operatorname{dist}\left(\phi_{\varepsilon}(\tau), \phi(\tau)\right)=o(1)$

(iii) there exist $0=\tau_{1}^{\varepsilon}<\tau_{2}^{\varepsilon}<\ldots<\tau_{\varepsilon}^{\varepsilon}=1$ with $\max _{i}\left[\tau_{i}^{\varepsilon}-\tau_{i-1}^{\varepsilon}\right]=o$ (1) such that $\max _{i}\left|F_{\varepsilon}\left(\phi_{\varepsilon}\left(\tau_{i}^{\varepsilon}\right)\right)-F\left(\phi\left(\tau_{i}^{\varepsilon}\right)\right)\right|=o(1)$ and $F_{\varepsilon}\left(\phi_{\varepsilon}(\tau)\right)$ is between $F_{\varepsilon}\left(\phi_{\varepsilon}\left(\tau_{i}^{\varepsilon}\right)\right)$ and $F_{\varepsilon}\left(\phi_{\varepsilon}\left(\tau_{i+1}^{\varepsilon}\right)\right)$ for $\tau \in\left(\tau_{i}^{\varepsilon}, \tau_{i+1}^{\varepsilon}\right)$, up to o(1); i.e., there exist infinitesimal $\beta_{n}>0$ such that

$$
\min \left\{F_{\varepsilon}\left(\phi_{\varepsilon}\left(\tau_{i}^{\varepsilon}\right)\right), F_{\varepsilon}\left(\phi_{\varepsilon}\left(\tau_{i+1}^{\varepsilon}\right)\right)\right\}-\beta_{n} \leq F_{\varepsilon}\left(\phi_{\varepsilon}(\tau)\right) \leq \max \left\{F_{\varepsilon}\left(\phi_{\varepsilon}\left(\tau_{i}^{\varepsilon}\right)\right), F_{\varepsilon}\left(\phi_{\varepsilon}\left(\tau_{i+1}^{\varepsilon}\right)\right)\right\}+\beta_{n}
$$

Then $\left(F_{\varepsilon}+G\right)$ is stable relative to $(E+G)$ for every continuous $G$ such that $\left(F_{\varepsilon}+G\right)$ is coercive.

Proof. Suppose that $u$ has a $\delta$-slide $\phi$ for $F+G$ (and therefore a $\left(\delta-\delta^{\prime}\right)$-slide for some $\left.\delta^{\prime}>0\right)$ and $u_{\varepsilon} \rightarrow u$. Then we choose $\psi_{\varepsilon}, \phi_{\varepsilon}$ as in (i)-(iii) above and set $\phi_{\varepsilon}^{\prime}(\tau):=\psi_{\varepsilon}(\tau)$ for $\tau \in[0,1]$, and $\phi_{\varepsilon}^{\prime}(\tau):=\phi_{\varepsilon}(\tau-1)$ for $\tau>1$. We then consider $\tau_{1}<\tau_{2} \in[0, T]$. If $\tau_{1}, \tau_{2} \in[0,1]$, then

$$
F_{\varepsilon}\left(\phi_{\varepsilon}^{\prime}\left(\tau_{2}\right)\right)-F_{\varepsilon}\left(\phi_{\varepsilon}^{\prime}\left(\tau_{1}\right)\right)=F_{\varepsilon}\left(\psi_{\varepsilon}\left(\tau_{2}\right)\right)-F_{\varepsilon}\left(\psi_{\varepsilon}\left(\tau_{1}\right)\right) \leq o(1) .
$$

If $\tau_{1}, \tau_{2}>1$, then

$$
F_{\varepsilon}\left(\phi_{\varepsilon}^{\prime}\left(\tau_{2}\right)\right)-F_{\varepsilon}\left(\phi_{\varepsilon}^{\prime}\left(\tau_{1}\right)\right)=F_{\varepsilon}\left(\phi_{\varepsilon}\left(\tau_{2}\right)\right)-F_{\varepsilon}\left(\phi_{\varepsilon}\left(\tau_{1}\right)\right) \leq E\left(\phi\left(\tau_{j}^{\varepsilon}\right)\right)-E\left(\phi\left(\tau_{i}^{\varepsilon}\right)\right)+o(1)
$$

for some $\tau_{i}^{\varepsilon} \leq \tau_{j}^{\varepsilon}$. If $\tau_{1}<1<\tau_{2}$, then

$$
F_{\varepsilon}\left(\phi_{\varepsilon}^{\prime}\left(\tau_{2}\right)\right)-F_{\varepsilon}\left(\phi_{\varepsilon}^{\prime}\left(\tau_{1}\right)\right)=F_{\varepsilon}\left(\phi_{\varepsilon}\left(\tau_{2}\right)\right)-F_{\varepsilon}\left(\psi_{\varepsilon}\left(\tau_{1}\right)\right) \leq E\left(\phi\left(\tau_{i}^{\varepsilon}\right)\right)-E(\phi(0))+o(1)
$$


for some $\tau_{i}^{\varepsilon}$, so that in any case

$$
\begin{aligned}
\left(F_{\varepsilon}\left(\phi_{\varepsilon}^{\prime}\left(\tau_{2}\right)\right)+\right. & \left.G\left(\phi_{\varepsilon}^{\prime}\left(\tau_{2}\right)\right)\right)-\left(F_{\varepsilon}\left(\phi_{\varepsilon}^{\prime}\left(\tau_{1}\right)\right)+G\left(\phi_{\varepsilon}^{\prime}\left(\tau_{1}\right)\right)\right) \\
& \leq\left(E\left(\phi\left(\tau_{j}\right)\right)+G\left(\phi\left(\tau_{j}\right)\right)\right)-\left(E\left(\phi\left(\tau_{i}\right)\right)+G\left(\phi\left(\tau_{i}\right)\right)\right)+o(1) \\
& <\delta-\delta^{\prime}+o(1)
\end{aligned}
$$

for some $\tau_{i} \leq \tau_{j}$, where we used the continuity of $G$ together with (ii) and (iii), as well as the fact that $\phi$ is a $\delta$-slide for $u$. The same argument gives

$$
\left(F_{\varepsilon}+G\right)\left(\phi_{\varepsilon}^{\prime}(1)\right)-\left(F_{\varepsilon}+G\right)\left(\phi_{\varepsilon}^{\prime}(0)\right) \leq(E+G)(\phi(1))-(E+G)(\phi(0))+o(1),
$$

so that $\phi_{\varepsilon}^{\prime}$ is a $\delta$-slide for $F_{\varepsilon}+G$, for $\varepsilon$ sufficiently small.

\subsection{Delta-stable evolution}

The notion of $\delta$-slide (or some of its modification) can be used to define evolutions in a similar way as in the case of quasi static motion, in cases when the presence of energy barriers may be relevant in the model under consideration. To that end, one can proceed by discrete approximation as in Remark 2.2.6:

- with fixed $\mathcal{F}(t, U)$ and $\mathcal{D}(U)$ energy and dissipation as in Section 2.2, time step $\tau>0$ and maximal barrier height $\delta>0$, define $U_{k}^{\tau}$ recursively by setting $U_{0}^{\tau}=U_{0}$, and choosing $U_{k}^{\tau}$ as a solution of the minimum problem

$$
\min _{U}\left\{\mathcal{F}(\tau k, U)+\mathcal{D}\left(U-U_{k-1}^{\tau}\right)\right\}
$$

on the class of $U$ such that there exists a path $\phi$ from $U_{k-1}^{\tau}$ to $U$ such that

$$
\mathcal{F}(\tau k, \phi(t))+\mathcal{D}\left(\phi(s)-U_{k-1}^{\tau}\right) \leq \mathcal{F}(\tau k, \phi(s))+\mathcal{D}\left(\phi(s)-U_{k-1}^{\tau}\right)+\delta
$$

if $0 \leq s<t \leq 1$.

- define the continuous trajectory $U^{\delta, \tau}(t)=U_{\lfloor t / \tau\rfloor}^{\tau}$;

- define the $\delta$-stable evolutions as the limits $U^{\delta}$ of (subsequences of) $U^{\delta, \tau}$ (which exists under suitable assumptions).

In order to ensure the existence of the minimizer $U_{k}^{\tau}$ some additional properties of the functionals

$$
F_{k}(U)=\mathcal{F}(\tau k, U)+\mathcal{D}\left(U-U_{k-1}^{\tau}\right)
$$

must be assumed; namely, that if $U_{j}$ is a sequence converging to $\bar{U}$ and $F_{k}\left(U_{j}\right)<F_{k}\left(U_{k-1}^{\tau}\right)-$ $C$ for some positive constant $C$ such that there exists paths $\phi_{j}$ from $U_{k-1}^{\tau}$ to $U_{j}$ satisfying (5.2) then there exists a path $\phi$ satisfying (5.2) from $U_{k-1}^{\tau}$ to $\bar{U}$. 
Remark 5.4.1 It must be noted that stable evolution gives a different notion from the global minimization approach even when $\mathcal{D}=0$, in which case the quasistatic approach just gives a parameterized choice of minimizers of $F(t, \cdot)$.

As a simple example take the one-dimensional energy

$$
F(t, x)=\min \left\{x^{2},(x-1)^{2}\right\}-2 t x,
$$

and $x_{0}=0$. Then the trajectory of parameterized minimizers of $F(t, x)$ from $x_{0}$ is

$$
u(t)= \begin{cases}0 & \text { if } t=0 \\ 1+t & \text { if } t>0 .\end{cases}
$$

On the contrary, the limit $\bar{u}$ as $\delta \rightarrow 0$ of the corresponding $\delta$-stable evolutions $u_{\delta}(t)$ is

$$
\bar{u}(t)= \begin{cases}t & \text { if } t<\frac{1}{2} \\ 1+t & \text { if } t \geq \frac{1}{2}\end{cases}
$$

Example 5.4.2 (the long-bar paradox in Fracture Mechanics) As shown in Remark 2.3.2, for one-dimensional fracture problems with applied boundary displacement; i.e., for the energies and dissipations

$$
\mathcal{F}(t, u)=\int_{0}^{1}\left|u^{\prime}\right|^{2} d t, \quad \mathcal{D}(S(u))=\#(S(u))
$$

defined on piecewise- $H^{1}$ functions $u$ with $u\left(0^{-}\right)=0$ and $u\left(1^{+}\right)=t$ (and $S(u)$ denotes the set of discontinuity points of $u$ ), fracture is brutal and appears at a critical value of the displacement $t$. If instead of a bar of unit length we take a bar of length $L$ and we consider the normalized boundary conditions $u(0-)=0$ and $u\left(L^{+}\right)=t L$, then the critical value for fracture is $t=\frac{1}{\sqrt{L}}$ for which the energy of the (unfractured) linear solution $u(t)=t x$ equals the energy of a piecewise-constant solution with one discontinuity. In other words a long bar fractures at lower values of the strain (the gradient of the linear solution). In order to overcome this drawback of the theory one may consider $\delta$-stable evolutions, or, rather, a small variation from it necessary due to the fact that the domains of the functionals $\mathcal{F}(t, \cdot)$ are disjoint for different $t$. In the iterated minimization scheme above we consider minimization among functions $u$ such that there exists a $L^{2}$-continuous path $\phi$ from the elastic solution $u_{k \tau}(x)=k \tau x$ (we again consider only the case $L=1$ ) to $u$ such that $\phi(t)(0)=0, \phi(t)(1)=k \tau$ for all $t$ and

$$
\mathcal{F}(\tau k, \phi(t))+\#(S(\phi(t)) \leq \mathcal{F}(\tau k, \phi(s))+\#(S(\phi(s))+\delta
$$


if $0 \leq s<t \leq 1$. This set of $u$ is contained in $H^{1}$. Indeed, otherwise there would be a $\bar{t} \in(0,1]$ such that $\#\left(S\left(\phi\left(t_{j}\right)\right) \geq 1\right.$ for a non-increasing sequence of $t_{j}$ converging to $t$, and $\phi(t) \in H^{1}$ for $t \leq \bar{t}$. By the lower semicontinuity of $\mathcal{F}$ and the minimality of $u_{k \tau}$ we have

$$
\mathcal{F}\left(k \tau, u_{k \tau}\right) \leq \mathcal{F}(k \tau, \phi(\bar{t})) \leq \mathcal{F}\left(k \tau, \phi\left(t_{j}\right)\right)+o(1),
$$

which gives

$$
\mathcal{F}(k \tau, \phi(0))+1 \leq \mathcal{F}\left(k \tau, \phi\left(t_{j}\right)\right)+\#\left(S\left(\phi\left(t_{j}\right)\right)+o(1)\right.
$$

contradicting (5.3) for $s=0$ and $t=t_{j}$ if $\delta<1$.

We conclude that for all $k$ the minimizer is exactly $u_{k \tau}$, and we may pass to the limit obtaining the elastic solution $u(t, x)=t x$. As a conclusion we have that no fracture appears, and this conclusion is clearly independent of the length of the bar.

\subsection{References to Chapter 5}

The notion of stable points has been introduced by Larsen in [32], where also stable fracture evolution has been studied; in particular there it is shown that the scheme in Section 5.4 can be applied to Griffith fracture energies.

The notions of stability for sequences of functionals have been analyzed by Braides and Larsen in [15], and are further investigated by Focardi in [23]. 


\section{Chapter 6}

\section{Minimizing movements}

\subsection{An energy-driven implicit-time discretization}

We now introduce a notion of energy-based motion which generalizes an implicit-time scheme for the approximation of solutions of gradient flows to general (also non differentiable) energies. We will use the terminology of minimizing movements, introduced by De Giorgi, even though we will not use the precise notation used in the literature.

Definition 6.1.1 (minimizing movements) Let $X$ be a separable Hilbert space and let $F: X \rightarrow[0,+\infty]$ be coercive and lower semicontinuous. Given $x_{0}$ and $\tau>0$ we define recursively $x_{k}$ as a minimizer for the problem

$$
\min \left\{F(x)+\frac{1}{2 \tau}\left\|x-x_{k-1}\right\|^{2}\right\}
$$

and the piecewise-constant trajectory $u^{\tau}:[0,+\infty) \rightarrow X$ given by

$$
u^{\tau}(t)=x_{\lfloor t / \tau\rfloor} .
$$

$A$ minimizing movement for $F$ from $x_{0}$ is any limit of a subsequence $u^{\tau_{j}}$ uniform on compact sets of $[0,+\infty)$.

As in the rest of these notes we are not focusing on the general topological assumptions on function spaces and convergences. In this definition we have taken $F \geq 0$ and $X$ Hilbert for the sale of simplicity. More in general, we can take $X$ a metric space and the (power of the) distance in place of the squared norm. In addition the topology on $X$ with respect to which $F$ is lower semicontinuous can be weaker than the one of the related distance.

Remark 6.1.2 A heuristic explanation of the definition above is given when $F$ is smooth. In this case, with the due notation, a minimizer for (6.1) solves the equation

$$
\frac{x_{k}-x_{k-1}}{\tau}=-\nabla F\left(u_{k}\right)
$$


i.e., $u^{\tau}$ solves the equation

$$
\frac{u^{\tau}(t)-u^{\tau}(t-\tau)}{\tau}=-\nabla F\left(u^{\tau}(t)\right)
$$

If we may pass to the limit in this equation as $u^{\tau} \rightarrow u$ then

$$
\frac{\partial u}{\partial t}=-\nabla F(u)
$$

This is easily shown if $X=\mathbb{R}^{n}$ and $F \in C^{2}\left(\mathbb{R}^{n}\right)$. In this case by taking any $\varphi \in$ $C_{0}^{\infty}\left((0, T) ; \mathbb{R}^{n}\right)$ we have

$$
-\int_{0}^{T}\left\langle\nabla F\left(u^{\tau}\right), \varphi\right\rangle d t=\int_{0}^{T}\left\langle\frac{u^{\tau}(t)-u^{\tau}(t-\tau)}{\tau}, \varphi\right\rangle d t=-\int_{0}^{T}\left\langle u^{\tau}(t), \frac{\varphi(t)-\varphi(t+\tau)}{\tau}\right\rangle d t
$$

from which, passing to the limit

$$
\int_{0}^{T}\langle\nabla F(u), \varphi\rangle d t=\int_{0}^{T}\left\langle u, \varphi^{\prime}\right\rangle d t
$$

i.e., (6.5) is satisfied in the sense of distributions, and hence in the classical sense.

Remark 6.1.3 (stationary solutions) Let $x_{0}$ be a local minimizer for $F$, then the only minimizing movement for $F$ from $x_{0}$ is the constant function $u(t)=x_{0}$.

Indeed, if $x_{0}$ is a minimizer for $F$ when $\left\|x-x_{0}\right\| \leq \delta$ by the positiveness of $F$ it is the only minimizer of $F(x)+\frac{1}{2 \tau}\left\|x-x_{0}\right\|^{2}$ for $\tau \leq \delta^{2} / F\left(x_{0}\right)$ if $F\left(x_{0}\right)>0$ (any $\tau$ if $F\left(x_{0}\right)=0$ ). So that $x_{k}=x_{0}$ for all $k$ for these $\tau$.

Proposition 6.1.4 (existence of minimizing movements) For all $F$ and $x_{0}$ as above there exists a minimizing movement $u \in C^{1 / 2}([0,+\infty) ; X)$.

Proof. By the coerciveness and lower semicontinuity of $F$ we obtain that $u_{k}$ are well defined for all $k$. Moreover, since

$$
F\left(x_{k}\right)+\frac{1}{2 \tau}\left\|x_{k}-x_{k-1}\right\|^{2} \leq F\left(x_{k-1}\right)
$$

we have $F\left(x_{k}\right) \leq F\left(x_{k-1}\right)$ and

$$
\left\|x_{k}-x_{k-1}\right\|^{2} \leq 2 \tau\left(F\left(x_{k-1}\right)-F\left(x_{k}\right)\right),
$$


so that for $t>s$

$$
\begin{aligned}
\left\|u^{\tau}(t)-u^{\tau}(s)\right\| & \leq \sum_{k=\lfloor s / \tau\rfloor+1}^{\lfloor t / \tau\rfloor}\left\|x_{k}-x_{k-1}\right\| \\
& \leq \sqrt{\lfloor t / \tau\rfloor-\lfloor s / \tau\rfloor} \sqrt{\sum_{k=\lfloor s / \tau\rfloor+1}^{\lfloor t / \tau\rfloor}\left\|x_{k}-x_{k-1}\right\|^{2}} \\
& \leq \sqrt{\lfloor t / \tau\rfloor-\lfloor s / \tau\rfloor} \sqrt{2 \tau \sum_{k=\lfloor s / \tau\rfloor+1}^{\lfloor t / \tau\rfloor}\left(F\left(x_{k-1}\right)-F\left(x_{k}\right)\right)} \\
& =\sqrt{\lfloor t / \tau\rfloor-\lfloor s / \tau\rfloor} \sqrt{2 \tau\left(F\left(x_{\lfloor s / \tau\rfloor}\right)-F\left(x_{\lfloor t / \tau\rfloor}\right)\right)} \\
& \leq \sqrt{2 F\left(x_{0}\right)} \sqrt{\tau(\lfloor t / \tau\rfloor-\lfloor s / \tau\rfloor)} \\
& \leq \sqrt{2 F\left(x_{0}\right)} \sqrt{t-s+\tau}
\end{aligned}
$$

This shows that the functions $u^{\tau}$ are (almost) equicontinuous and equibounded in $C([0,+\infty) ; X)$. Hence, they converge uniformly. Moreover, passing to the limit we obtain

$$
\|u(t)-u(s)\| \leq \sqrt{2 F\left(x_{0}\right)} \sqrt{|t-s|}
$$

so that $u \in C^{1 / 2}([0,+\infty) ; X)$.

Remark 6.1.5 (growth conditions) The positiveness of $F$ can be substituted by the requirement that for all $\bar{x}$ the functionals

$$
x \mapsto F(x)+\frac{1}{2 \tau}\|x-\bar{x}\|^{2}
$$

be bounded from below; i.e., that there exists $C>0$ such that

$$
x \mapsto F(x)+C\|x-\bar{x}\|^{2}
$$

be bounded from below.

Example 6.1.6 (non-uniqueness of minimizing movements) If $F$ is not $C^{2}$ we may have more than one minimizing movement.

(i) Bifurcation at times with multiple minimizers. A simple example is $F(x)=-\frac{1}{\alpha}|x|^{\alpha}$ with $0<\alpha<2$, which is not $C^{2}$ at $x=0$. In this case, for $x_{0}=0$ we have a double choice for minimum problem (6.1); i.e.,

$$
x_{1}= \pm \tau^{1 /(2-\alpha)}
$$


Once $x_{1}$ is chosen all other valued are determined, and it can be seen that either $x_{k}>0$ for all $k$ or $x_{k}<0$ for all $k$ (for $\alpha=1$, e.g., we have $x_{k}= \pm k \tau$ ), and that in the limit we have the two solutions of

$$
\left\{\begin{array}{l}
u^{\prime}=|u|^{(\alpha-2)} u \\
u(0)=0
\end{array}\right.
$$

with $u(t) \neq 0$ for $t>0$. Note in particular that we do not have the trivial solution $u=0$. In this example we do not have to pass to a subsequence of $\tau$.

(i) Different movements depending on subsequences of $\tau$. Discrete trajectories can be different depending on the time step $\tau$. We give an explicit example, close in spirit to the previous one. In this example the function $F$ is asymmetric, so that $x_{1}$ is unique but may take positive or negative values depending on $\tau$.

We define $F$ as the Lipschitz function taking value 0 at $x=0$, for $x>0$

$$
F^{\prime}(x)= \begin{cases}-1 & \text { if } 2^{-2 k-1}<x<2^{-2 k}, k \in \mathbb{N} \\ -2 & \text { otherwise for } x>0\end{cases}
$$

and $F^{\prime}(x)=3+F^{\prime}(-x)$ for $x<0$. It is easily seen that for $x_{0}=0$ we may have a unique minimizer $x_{1}$ with $x_{1}>0$ or $x_{1}<0$ depending on $\tau$. In particular we have $x_{1}=-2^{-2 k}<0$ for $\tau=2^{-2 k-1}$ and $x_{1}=2^{-2 k+1}>0$ for $\tau=2^{-2 k}$. In the two cases we then have again the solutions to

$$
\left\{\begin{array}{l}
u^{\prime}=-F(u) \\
u(0)=0
\end{array}\right.
$$

with $u(t)<0$ for all $t>0$ or $u(t)>0$ for all $t>0$, respectively.

Example 6.1.7 (heat equation) Taking $X=L^{2}(\Omega)$ and the Dirichlet integral $F(u)=$ $\frac{1}{2} \int_{\Omega}|\nabla u|^{2} d x$, with fixed $u_{0} \in H^{1}(\Omega)$ and $\tau>0$ we can solve iteratively

$$
\min \left\{\frac{1}{2} \int_{\Omega}|\nabla u|^{2} d x+\frac{1}{2 \tau} \int_{\Omega}\left|u-u_{k-1}\right|^{2} d x\right\},
$$

whose unique minimizer $u_{k}$ solves the Euler-Lagrange equation

$$
\frac{u_{k}-u_{k-1}}{\tau}=\Delta u_{k}, \quad \frac{\partial u_{k}}{\partial \nu}=0 \text { on } \partial \Omega,
$$

where $\nu$ is the inner normal to $\Omega$. We then set $u^{\tau}(x, t)=u_{\lfloor t / \tau\rfloor}(x)$, which converges, up to subsequences, to $u(x, t)$. We can then pass to the limit in $(6.7)$ in the sense of distributions to obtain the heat equation

$$
\frac{\partial u}{\partial t}=\Delta u, \quad \frac{\partial u}{\partial \nu}=0 \text { on } \partial \Omega
$$

combined with the initial datum $u(x, 0)=u_{0}(x)$. Due to the uniqueness of the solution to the heat equation we also obtain that the whole sequence converges as $\tau \rightarrow 0$. 
Example 6.1.8 (one-dimensional fracture energies) In dimension one, we still consider $X=L^{2}(0,1)$ and the Griffith (or Mumford-Shah) energy

$$
F(u)=\frac{1}{2} \int_{0}^{1}\left|u^{\prime}\right|^{2} d x+\#(S(u))
$$

with domain piecewise- $H^{1}$ functions. We fix $u_{0}$ piecewise- $H^{1}$ and $\tau>0$. In this case we solve iteratively

$$
\min \left\{\frac{1}{2} \int_{0}^{1}\left|u^{\prime}\right|^{2} d x+\#(S(u))+\frac{1}{2 \tau} \int_{0}^{1}\left|u-u_{k-1}\right|^{2} d x\right\} .
$$

This problem is not convex, and may have multiple minimizers. Nevertheless in this simpler case we can prove iteratively that for $\tau$ small enough we have $S\left(u_{k}\right)=S\left(u_{0}\right)$ for all $k$, and hence reduce to the independent iterated minimization problems of the Dirichlet integral on each component of $(0,1) \backslash S\left(u_{0}\right)$, giving the heat equation in $(0,1) \backslash S\left(u_{0}\right)$ with Neumann boundary conditions on 0,1 and $S\left(u_{0}\right)$. The description holds until the first time $T$ such that $u\left(x^{-}, T\right)=u\left(x^{+}, T\right)$ at some point $x \in S(u(\cdot, T))$.

We check this with some simplifying hypotheses:

(1) that $\int_{0}^{1}\left|u_{0}^{\prime}\right|^{2} d x<2$. This implies that $\#\left(S\left(u_{k}\right)\right) \leq \# S\left(u_{0}\right)$ since by the monotonicity of the energy we have $\#\left(S\left(u_{k}\right)\right) \leq F\left(u_{k}\right) \leq F\left(u_{0}\right)<\# S\left(u_{0}\right)+1$. This hypothesis can be removed with a localization argument;

(2) that there exists $\eta>0$ such that $\left|u_{0}(x)-u_{0}\left(x^{\prime}\right)\right| \geq \eta$ if $\left(x, x^{\prime}\right) \cap S\left(u_{0}\right) \neq \emptyset$. This will imply that $\left|u_{k}\left(x^{+}\right)-u_{k}\left(x^{-}\right)\right| \geq \eta$ at all $x \in S\left(u_{k}\right)$ so that $T=+\infty$ in the notation above.

Furthermore, we suppose that $S\left(u_{0}\right)=\left\{x_{0}\right\}$ (a single point) and $u_{0}\left(x_{0}^{+}\right)>u_{0}\left(x_{0}^{-}\right)$, for simplicity of notation.

We reason by induction. We first examine the properties of $u_{1}$; checking that it has a jump point close to $x_{0}$. Suppose otherwise that there exists $\delta>0$ such that $u \in$ $H^{1}\left(x_{0}-\delta, x_{0}+\delta\right)$. We take $\delta$ small enough so that

$$
\begin{aligned}
& u_{0}(x) \leq u\left(x_{0}^{-}\right)+\frac{1}{4}\left(u\left(x_{0}^{+}\right)-u\left(x_{0}^{-}\right)\right) \text {for } x_{0}-\delta<x<x_{0} \\
& u_{0}(x) \geq u\left(x_{0}^{+}\right)-\frac{1}{4}\left(u\left(x_{0}^{+}\right)-u\left(x_{0}^{-}\right)\right) \text {for } x_{0}<x<x_{0}+\delta .
\end{aligned}
$$

In this case

$$
\begin{aligned}
& \frac{1}{2} \int_{x_{0}-\delta}^{x_{0}+\delta}\left|u_{1}^{\prime}\right|^{2} d x+\frac{1}{2 \tau} \int_{x_{0}-\delta}^{x_{0}+\delta}\left|u_{1}-u_{0}\right|^{2} d x \\
\geq & \frac{1}{2} \min \left\{\int_{0}^{\delta}\left|v^{\prime}\right|^{2} d x+\frac{1}{\tau} \int_{0}^{\delta}|v|^{2} d x: v(\delta)=\frac{1}{4}\left(u\left(x_{0}^{+}\right)-u\left(x_{0}^{-}\right)\right)\right\} \\
= & \frac{\left(u\left(x_{0}^{+}\right)-u\left(x_{0}^{-}\right)\right)^{2}}{8 \sqrt{\tau}} \tanh \left(\frac{\delta}{\sqrt{\tau}}\right),
\end{aligned}
$$


the last equality easily obtained by computing the solution of the Euler-Lagrange equation. This shows that for each such $\delta>0$ fixed we have $\left(x_{0}-\delta, x_{0}+\delta\right) \cap S\left(u_{1}\right) \neq \emptyset$ for $\tau$ sufficiently small. Note that the smallness of $\tau$ depends only on the size of $u\left(x_{0}^{+}\right)-u\left(x_{0}^{-}\right)$(which is larger than $\eta)$. Since $\# S\left(u_{1}\right) \leq \# S\left(u_{0}\right)$ we then have $\left(x_{0}-\delta, x_{0}+\delta\right) \cap S\left(u_{1}\right)=\left\{x_{1}\right\}$; we may suppose that $x_{1} \geq x_{0}$.

We now check that $x_{1}=x_{0}$. Suppose otherwise; then note that by the Hölder continuity of $u_{1}$ we have that for $\delta$ small enough (depending only on the size of $u\left(x_{0}^{+}\right)-u\left(x_{0}^{-}\right)$) we have

$$
u_{1}(x) \leq u_{0}\left(x_{0}^{-}\right)+\frac{1}{8}\left(u\left(x_{0}^{+}\right)-u\left(x_{0}^{-}\right)\right) \text {for } x_{0}<x<x_{1}
$$

and

$$
u_{1}\left(x_{1}^{+}\right) \geq u_{0}\left(x_{1}^{+}\right)-\frac{1}{8}\left(u\left(x_{0}^{+}\right)-u\left(x_{0}^{-}\right)\right) .
$$

We may then consider the function $\widetilde{u}$ coinciding with $u_{1}$ on $\left(0, x_{0}\right)$, with $\widetilde{u}^{\prime}-U_{1}^{\prime}$ and $S(\widetilde{u})=\left\{x_{0}\right\}$. Then $F(\widetilde{u})=F\left(u_{1}\right)$ and

$$
\int_{0}^{1}\left|\widetilde{u}-u_{0}\right|^{2} d x<\int_{0}^{1}\left|u_{1}-u_{0}\right|^{2} d x
$$

contradicting the minimality of $u_{1}$. Hence, we have $S\left(u_{1}\right)=S\left(u_{0}\right)$.

Note that $u_{1}$ is obtained by separately minimizing the problems with the Dirichlet integral on $\left(0, x_{0}\right)$ and $\left(x_{0}, 1\right)$, and in particular that on each such interval sup $u_{1} \leq \sup u_{0}$ and $\inf u_{1} \geq \inf u_{0}$, so that the condition that $\left|u_{1}(x)-u_{1}\left(x^{\prime}\right)\right| \geq \eta$ if $\left(x, x^{\prime}\right) \cap S\left(u_{1}\right) \neq \emptyset$ still hold. This shows that we can iterate the scheme obtaining $u_{k}$ which satisfy $\inf u_{0} \leq$ $u_{k} \leq \sup u_{0}$ on each component of $(0,1) \backslash S\left(u_{0}\right)$ and $u_{k}^{\prime}=0$ on 0,1 and $S\left(u_{0}\right)$. In particular $\left|u_{k}^{+}-u_{k}^{-}\right| \geq \eta$ on $S\left(u_{0}\right)$, which shows that the limit satisfies the heat equation with Neumann conditions on $S\left(u_{0}\right)$ for all times.

Remark 6.1.9 (higher-dimensional fracture energies: an open problem) In dimension higher than one, we may apply the minimizing-movement scheme to the Mumford-Shah functional (which can also be interpreted as a Griffith energy in anti-plane setting). To this scheme we may also add the constraint of increasing crack in the same way as in Section 2.3. We then obtain a minimizing movement $u$ in $L^{2}(\Omega)$ from an initial datum $u_{0}$. The solution $u$ solves a weak form of the heat equation almost everywhere in $\Omega$, and in some cases it can be proved that Neumann boundary conditions still holds on $S(u)$, but it remains open the characterization of the (possible) motion of the crack set. As compared with the Francfort and Marigo quasistatic theory, here an analog of the Francfort-Larsen transfer lemma is missing. 


\subsection{Time-dependent minimizing movements}

We can generalize the definition of minimizing movement to include forcing terms or varying boundary conditions, by considering time-parameterized energies $F(x, t)$ and, given $\tau$ and an initial datum $x_{0}$, define $x_{k}$ recursively by choosing $x_{k}$ as a minimizer of

$$
\min \left\{F(x, k \tau)+\frac{1}{2 \tau}\left\|x-x_{k-1}\right\|^{2}\right\}
$$

and eventually define $u^{\tau}(t)=x_{\lfloor t / \tau\rfloor}$. We may define, up to subseqences, a limit $u$ of $u^{\tau}$ as $\tau \rightarrow 0$ if some technical hypothesis is added to $F$. One such is that in the scheme above

$$
F\left(u_{k}, k \tau\right)+\frac{1}{2 \tau}\left\|x_{k}-x_{k-1}\right\|^{2} \leq(1+C \tau) F\left(u_{k-1},(k-1) \tau\right)+C \tau,
$$

for some $C$ (at least if $k \tau$ remains bounded). With such a condition we can repeat the convergence argument as for the time-independent case and obtain a limit minimizing movement $u$.

Indeed, with such a condition we have

$$
\left\|x_{k}-x_{k-1}\right\|^{2} \leq 2 \tau\left((1+C \tau) F\left(u_{k-1},(k-1) \tau\right)-F\left(u_{k}, k \tau\right)+C \tau\right)
$$

and the inequality (for $\tau$ small enough)

$$
F\left(u_{k}, k \tau\right) \leq(1+C \tau) F\left(u_{k-1},(k-1) \tau\right)+C \tau \leq(1+C \tau)\left(F\left(u_{k-1},(k-1) \tau\right)+1\right)
$$

that implies that $F\left(u_{k}, k \tau\right)$ is equibounded for $k \tau$ bounded. We fix $T>0$; from (6.11) we obtain (for $0 \leq s \leq t \leq T$ )

$$
\begin{aligned}
\left\|u^{\tau}(t)-u^{\tau}(s)\right\| & \leq \sum_{k=\lfloor s / \tau\rfloor+1}^{\lfloor t / \tau\rfloor}\left\|x_{k}-x_{k-1}\right\| \\
& \leq \sqrt{\lfloor t / \tau\rfloor-\lfloor s / \tau\rfloor} \sqrt{\sum_{k=\lfloor s / \tau\rfloor+1}^{\lfloor t / \tau\rfloor}\left\|x_{k}-x_{k-1}\right\|^{2}} \\
& \leq \sqrt{\lfloor t / \tau\rfloor-\lfloor s / \tau\rfloor} \sqrt{\left.2 \tau \sum_{k=\lfloor s / \tau\rfloor+1}^{\lfloor t / \tau\rfloor}\left(F\left(x_{k-1},(k-1) \tau\right)-F\left(x_{k}, k \tau\right)+C_{T} \tau\right)\right)} \\
& =\sqrt{\lfloor t / \tau\rfloor-\lfloor s / \tau\rfloor} \sqrt{\left.2 \tau F\left(x_{0}, 0\right)+C_{T} \tau(\lfloor t / \tau\rfloor-\lfloor s / \tau\rfloor)\right)} \\
& \leq \sqrt{2 F\left(x_{0}, 0\right)(t-s+\tau)+C_{T}(t-s+\tau)^{2}},
\end{aligned}
$$

which gives an equicontinuity condition sufficient to pass to the limit as $\tau \rightarrow 0$.

Note that from (6.12) for $s<t$ we obtain the estimate

$$
F\left(u^{\tau}(t),\lfloor t / \tau\rfloor \tau\right) \leq e^{C(t-s+\tau)}\left(F\left(u^{\tau}(s),\lfloor s / \tau\rfloor \tau\right)+C \tau\right) .
$$


Example 6.2.1 (heat equation with varying boundary conditions) We can take

$$
F(u, t)=\frac{1}{2} \int_{0}^{1}\left|u^{\prime}\right|^{2} d x
$$

with domain all $H^{1}$-functions with $u(0)=0$ and $u(1)=t$.

Then if we can test the problem defining $u_{k}$ with the function $\tilde{u}=u_{k-1}+\tau x$. We then have

$$
\begin{aligned}
F\left(u_{k}, k \tau\right)+\frac{1}{2 \tau}\left\|u_{k}-u_{k-1}\right\|^{2} & \leq F(\tilde{u}, k \tau)+\frac{1}{2 \tau}\left\|\tilde{u}-u_{k-1}\right\|^{2} \\
& =\frac{1}{2} \int_{0}^{1}\left|u_{k-1}^{\prime}+\tau\right|^{2} d x+\frac{1}{6} \tau^{2} \\
& \leq(1+\tau) \frac{1}{2} \int_{0}^{1}\left|u_{k-1}^{\prime}\right|^{2} d x+\tau+\frac{1}{6} \tau^{2} \\
& \leq(1+\tau) \frac{1}{2} F\left(u_{k-1},(k-1) \tau\right)+\left(1+\frac{1}{6} \tau\right) \tau .
\end{aligned}
$$

which gives (6.10).

We then have the convergence of $u^{\tau}$ to the solution $u$ of the equation

$$
\left\{\begin{array}{l}
\frac{\partial u}{\partial t}=\frac{\partial^{2} u}{\partial x^{2}} \\
u(0, t)=0, u(1, t)=t \\
u(x, 0)=u_{0}(x) .
\end{array}\right.
$$

Clearly in this example we may take any Lipschitz function $g(t)$ in place of $t$ as boundary condition.

Example 6.2.2 (minimizing movements vs quasi static evolution for fracture) We can take

$$
F(u, t)=\frac{1}{2} \int_{0}^{1}\left|u^{\prime}\right|^{2} d x+\# S(u),
$$

with domain all piecewise- $H^{1}$-loc functions with $u(x)=0$ if $x \leq 0$ and $u(x)=t$ for $x \geq 1$, so that $S(u) \subset[0,1]$, and the fracture may also appear at the boundary points 0 and 1. As in the previous example we can test the problem defining $u_{k}$ with the function $\tilde{u}=u_{k-1}+\tau x$ since $\# S(\tilde{u})=\# S\left(u_{k-1}\right)$, to obtain $(6.10)$.

We consider the initial datum $u_{0}=0$. Note that the minimum problems for $F(\cdot, t)$ correspond to the definition of quasi static evolution in Remark 2.3.2. We now show that for problems (6.9) the solution does not develop fracture. 
Indeed, consider

$$
k_{\tau}=\min \left\{k: u_{k} \notin H_{\mathrm{loc}}^{1}(\mathbb{R})\right\},
$$

and suppose that $\tau k_{\tau} \rightarrow \bar{t} \in[0,+\infty)$. Then we have that $u^{\tau}$ converges on $[0, \bar{t}]$ to $u$ described by (6.14) in the previous example. Moreover we may suppose that

$$
\lim _{\tau \rightarrow 0} \int_{0}^{1}\left|u_{k_{\tau}-1}^{\prime}\right|^{2} d x=\int_{0}^{1}\left|u^{\prime}(x, \bar{t})\right|^{2} d x
$$

and since also $u_{k_{\tau}} \rightarrow u(\cdot, \bar{t})$ as $\tau \rightarrow 0$, we have

$$
\int_{0}^{1}\left|u^{\prime}(x, \bar{t})\right|^{2} d x \leq \liminf _{\tau \rightarrow 0} \int_{0}^{1}\left|u_{k_{\tau}}^{\prime}\right|^{2} d x
$$

We deduce that

$$
F\left(u_{k_{\tau}}, k_{\tau} \tau\right) \geq F\left(u_{k_{\tau}-1},\left(k_{\tau}-1\right) \tau\right)+1+o(1)
$$

as $\tau \rightarrow 0$, which contradicts (6.12).

From the analysis above we can compare various ways to define the evolutive response of a brittle elastic material to applied increasing boundary displacements (at least in a one-dimensional setting):

- (quasistatic motion) the response is purely elastic until a threshold (depending on the size of the specimen) is reached, after which we have brutal fracture;

- (stable evolution) the response is purely elastic, and corresponds to minimizing the elastic energy at fixed boundary displacement. No fracture occurs;

- (minimizing movement) in this case the solution does not develop fracture, but follows the heat equation with given boundary conditions.

\subsection{References to Chapter 6}

The terminology '(generalized) minimizing movement' has been introduced by De Giorgi in a series of papers devoted to mathematical conjectures (see [22]). We also refer to the original treatment by L. Ambrosio [3].

A theory of gradient flows in metric spaces using minimizing movements is described in the book by Ambrosio, Gigli and Savaré [7]. L. Ambrosio, N. Gigli and G. Savaré, Gradient flows in metric spaces and in the space of probability measures, Lectures in Mathematics ETH Zürich. Birkhäuser, Basel, 2005.

Minimizing movements for the Mumford-Shah functional in more that one space dimension (and hence also for the Griffith fracture energy) with the condition of increasing fracture have been defined by Ambrosio and Braides [4], and partly analyzed in a twodimensional setting by Chambolle and F. Doveri [20]. 


\section{Chapter 7}

\section{Minimizing movements along a sequence of functionals}

Gradient flows, and hence minimizing movements, trivially do not commute even with uniform convergence. As a simple example, take $X=\mathbb{R}$ and

$$
F_{\varepsilon}(x)=x^{2}-\rho \sin \left(\frac{x}{\varepsilon}\right),
$$

with $\rho=\rho_{\varepsilon} \rightarrow 0$ as $\varepsilon \rightarrow 0$, uniformly converging to $F(x)=x^{2}$. If also

$$
\varepsilon<<\rho,
$$

then for fixed $x_{0}$ the solutions $u_{\varepsilon}$ to the equation

$$
\left\{\begin{array}{l}
u_{\varepsilon}^{\prime}=-2 u_{\varepsilon}+\frac{\rho}{\varepsilon} \cos \left(\frac{u_{\varepsilon}}{\varepsilon}\right) \\
u_{\varepsilon}(0)=x_{0}
\end{array}\right.
$$

converge to the constant function $u_{0}(t)=x_{0}$ as $\varepsilon \rightarrow 0$, which does not solve

$$
\left\{\begin{array}{l}
u^{\prime}=-2 u \\
u(0)=x_{0}
\end{array}\right.
$$

This is easily seen by studying the stationary solutions of

$$
-2 x+\frac{\rho}{\varepsilon} \cos \left(\frac{x}{\varepsilon}\right)=0 .
$$

With the remark above in mind, in order to give a meaningful limit for the energydriven motion along a sequence of functionals it may be useful to vary the definition of minimizing movement as in the following section. 


\subsection{Minimizing movements along a sequence}

Definition 7.1.1 (minimizing movements along a sequence) Let $X$ be a separable Hilbert space, $F_{\varepsilon}: X \rightarrow[0,+\infty]$ equicoercive and lower semicontinuous and $x_{0}^{\varepsilon} \rightarrow x_{0}$ with

$$
F_{\varepsilon}\left(x_{0}^{\varepsilon}\right) \leq C<+\infty
$$

and $\tau_{\varepsilon}>0$ converging to 0 as $\varepsilon \rightarrow 0$. Fixed $\varepsilon>0$ we define recursively $x_{k}^{\varepsilon}$ as a minimizer for the problem

$$
\min \left\{F_{\varepsilon}(x)+\frac{1}{2 \tau}\left\|x-x_{k-1}^{\varepsilon}\right\|^{2}\right\}
$$

and the piecewise-constant trajectory $u^{\varepsilon}:[0,+\infty) \rightarrow X$ given by

$$
u^{\varepsilon}(t)=x_{\left\lfloor t / \tau_{\varepsilon}\right\rfloor} .
$$

$A$ minimizing movement for $F_{\varepsilon}$ from $x_{0}^{\varepsilon}$ is any limit of a subsequence $u^{\varepsilon_{j}}$ uniform on compact sets of $[0,+\infty)$.

After remarking that the Hölder continuity estimates in Proposition 6.1.4 only depend on the bound on $F_{\varepsilon}\left(x_{0}^{\varepsilon}\right)$, with the same proof we can show the following result.

Proposition 7.1.2 For every $F_{\varepsilon}$ and $x_{0}^{\varepsilon}$ as above there exist minimizing movements for $F_{\varepsilon}$ from $x_{0}^{\varepsilon}$ in $C^{1 / 2}([0,+\infty) ; X)$.

Remark 7.1.3 (growth conditions) The positiveness of $F_{\varepsilon}$ can be substituted by the requirement that for all $\bar{x}$ the functionals

$$
x \mapsto F_{\varepsilon}(x)+\frac{1}{2 \tau}\|x-\bar{x}\|^{2}
$$

be bounded from below; i.e., that there exists $C>0$ such that

$$
x \mapsto F_{\varepsilon}(x)+C\|x-\bar{x}\|^{2}
$$

be bounded from below.

Example 7.1.4 We give a simple example that shows how the limit minimizing movement may depend on the choice of the mutual behavior of $\varepsilon$ and $\tau$. We consider the functions

$$
F_{\varepsilon}(x)= \begin{cases}-x & \text { if } x \leq 0 \\ 0 & \text { if } 0 \leq x \leq \varepsilon \\ \varepsilon-x & \text { if } x \geq \varepsilon,\end{cases}
$$


which converge uniformly to $F(x)=-x$. Note the that the energies are not bounded from below, but their analysis falls within the framework in the previous remark. For this example a direct computation is immediately carried on. We consider a fixed initial datum $x_{0}$.

If $x_{0}>0$, then for $\varepsilon<x_{0}$ we have $x_{k}^{\varepsilon}=x_{k-1}^{\varepsilon}+\tau$ for all $k \geq 0$.

If $x_{0} \leq 0$ then we have $x_{k}^{\varepsilon}=x_{k-1}^{\varepsilon}+\tau$ if $x_{k-1}^{\varepsilon} \leq-\tau$. If $0 \geq x_{k-1}^{\varepsilon}>-\tau$ then $x_{k}^{\varepsilon}-x_{k-1}^{\varepsilon}$ is obtained by minimizing the function

$$
f(y)= \begin{cases}-y+\frac{1}{2 \tau} y^{2} & \text { if } 0 \leq y \leq-x_{k-1}^{\varepsilon} \\ x_{k-1}^{\varepsilon}+\frac{1}{2 \tau} y^{2} & \text { if }-x_{k-1}^{\varepsilon} \leq y \leq-x_{k-1}^{\varepsilon}+\varepsilon \\ \varepsilon-y+\frac{1}{2 \tau} y^{2} & \text { if } y \geq-x_{k-1}^{\varepsilon}+\varepsilon,\end{cases}
$$

whose minimizer is always $y=\tau+x_{k-1}^{\varepsilon}$ if $\varepsilon-x_{k-1}^{\varepsilon}>\tau$. In this case $x_{k}^{\varepsilon}=0$. If otherwise $\varepsilon-x_{k-1}^{\varepsilon} \leq \tau$ the other possible minimizer is $y=\tau$. We then have to compare the values

$$
f\left(-x_{k-1}^{\varepsilon}\right)=x_{k-1}^{\varepsilon}+\frac{1}{2 \tau}\left(x_{k-1}^{\varepsilon}\right)^{2}, \quad f(\tau)=\varepsilon-\frac{1}{2} \tau .
$$

We have three cases:

(a) $\varepsilon-\frac{1}{2} \tau>0$. In this case we have $x_{k}^{\varepsilon}=0$ (and this holds for all subsequent steps);

(b) $\varepsilon-\frac{1}{2} \tau<0$. In this case we either have $f(\tau)<f\left(-x_{k-1}^{\varepsilon}\right)$, in which case $x_{k}^{\varepsilon}=x_{k-1}^{\varepsilon}+\tau$ (and this then holds for all subsequent steps); otherwise $x_{k}^{\varepsilon}=0$ and $x_{k+1}^{\varepsilon}=x_{k}^{\varepsilon}+\tau$ (and this holds for all subsequent steps);

(c) $\varepsilon-\frac{1}{2} \tau=0$. If $x_{k-1}^{\varepsilon}<0$ then $x_{k}^{\varepsilon}=0$ (otherwise we already have $x_{k-1}^{\varepsilon}=0$ ). Then, since we have the two solutions $y=0$ and $y=\tau$, we have $x_{j}^{\varepsilon}=0$ for $k \leq j \leq k_{0}$ for some $k_{0} \in \mathbb{N} \cup+\infty$ and $x_{j}^{\varepsilon}=x_{j-1}^{\varepsilon}+\tau$ for $j>k_{0}$.

We can summarize the possible minimizing movements with initial datum $x_{0} \leq 0$ as follows:

(i) if $\tau<2 \varepsilon$ then the unique minimizing movement is $x(t)=\min \left\{x_{0}+t, 0\right\}$;

(ii) if $\tau>2 \varepsilon$ then the unique minimizing movement is $x(t)=x_{0}+t$;

(iii) if $\tau=2 \varepsilon$ then we have the minimizing movements $x(t)=\max \left\{\min \left\{x_{0}+t, 0\right\}, x_{1}+t\right\}$ for $x_{1} \leq x_{0}$.

For $x_{0}>0$ we always have the only minimizing movement $x(t)=x_{0}+t$.

\subsection{Commutability along 'fast-converging' sequences}

We now show that by suitably choosing the $\varepsilon-\tau$ regimes the minimizing movement along the sequence $F_{\varepsilon}$ from $x_{\varepsilon}$ converges to a minimizing movement for the limit $F$ from $x_{0}$ 
('fast-converging $\varepsilon$ '), while for other choices ('fast-converging $\tau$ ') the minimizing movement converges to a limit of minimizing movements for $F_{\varepsilon}$ as $\varepsilon \rightarrow 0$. The heuristic statement is that minimizing movements for all other regimes are 'trapped' between these two extrema.

Theorem 7.2.1 Let $F_{\varepsilon}$ be a equi-coercive sequence of (non-negative) lower-semicontinuous functionals on a Hilbert space $X \Gamma$-converging to $F$, let $x_{\varepsilon} \rightarrow x_{0}$. Then

(i) there exists a choice of $\varepsilon=\varepsilon(\tau)$ such that every minimizing movement along $F_{\varepsilon}$ (and with time-step $\tau$ ) with initial data $x_{\varepsilon}$ is a minimizing movement for $F$ from $x_{0}$ on $[0, T]$ for all $T$;

(ii) there exists a choice of $\tau=\tau(\varepsilon)$ such that every minimizing movement along $F_{\varepsilon}$ (and with time-step $\tau$ ) with initial data $x_{\varepsilon}$ is a the limit of a sequence of minimizing movements for $F_{\varepsilon}$ (for $\varepsilon$ fixed) from $x_{\varepsilon}$ on $[0, T]$ for all $T$.

Proof. (i) Note that if $y_{\varepsilon} \rightarrow y_{0}$ then the solutions of

$$
\min \left\{F_{\varepsilon}(x)+\frac{1}{2 \tau}\left\|x-y_{\varepsilon}\right\|^{2}\right\}
$$

converge to solutions of

$$
\min \left\{F(x)+\frac{1}{2 \tau}\left\|x-y_{0}\right\|^{2}\right\}
$$

since we have a continuously converging perturbation of a $\Gamma$-converging sequence.

Let now $x_{\varepsilon} \rightarrow x_{0}$. Let $\tau$ be fixed. We consider the sequence $\left\{x_{k}^{\tau, \varepsilon}\right\}$ defined by iterated minimization of $F_{\varepsilon}$ with initial point $x_{\varepsilon}$. Since $x_{\varepsilon} \rightarrow x_{0}$, up to subsequences we have $x_{1}^{\tau, \varepsilon} \rightarrow x_{1}^{\tau, 0}$, which minimizes

$$
\min \left\{F(x)+\frac{1}{2 \tau}\left\|x-x_{0}\right\|^{2}\right\} .
$$

The point $x_{2}^{\tau, \varepsilon}$ converge to $x_{2}^{\tau, 0}$. Since they minimize

$$
\min \left\{F_{\varepsilon}(x)+\frac{1}{2 \tau}\left\|x-x_{1}^{\tau, \varepsilon}\right\|^{2}\right\}
$$

and $x_{1}^{\tau, \varepsilon} \rightarrow x_{1}^{\tau, 0}$ their limit is a minimizer of

$$
\min \left\{F(x)+\frac{1}{2 \tau}\left\|x-x_{1}^{\tau, 0}\right\|^{2}\right\}
$$

This operation can be repeated iteratively, obtaining (upon subsequences) $x_{k}^{\tau, \varepsilon} \rightarrow x_{k}^{\tau, 0}$, and $\left\{x_{k}^{\tau, 0}\right\}$ iteratively minimizes $F$ with initial point $x_{0}$. Since up to subsequences the trajectories $\left\{x_{k}^{\tau, 0}\right\}$ converge to a minimizing movement for $F$ with initial datum $x_{0}$ the thesis follows by a diagonal argument.

(ii) For fixed $\varepsilon$ the piecewise-constant functions $u^{\varepsilon, \tau}(t)=x_{\lfloor t / \tau\rfloor}^{\varepsilon, \tau}$ converge uniformly to a minimizing movement $u^{\varepsilon}$ for $F_{\varepsilon}$ with initial datum $x_{\varepsilon}$. By compactness, these $u^{\varepsilon}$ converge uniformly to some function $u$ as $\varepsilon \rightarrow 0$. Again, a diagonal argument gives the thesis. 
Remark 7.2.2 Note that, given $x_{\varepsilon}$ and $F_{\varepsilon}$, if $F$ has more than one minimizing movement from $x_{0}$ then the approximation gives a choice criterion. As an example take $F(x)=-|x|$, $F_{\varepsilon}(x)=-|x+\varepsilon|$ and $x_{0}=x_{\varepsilon}=0$.

Remark 7.2.3 (the convex case) If all $F_{\varepsilon}$ are convex then it can be shown that actually the minimizing movement along the sequence $F_{\varepsilon}$ always coincides with the minimizing movement for their $\Gamma$-limit. This (exceptional) case will be dealt with in detail separately in Section 10.

Example 7.2.4 In dimension one we can take

$$
F_{\varepsilon}(x)=\frac{1}{2} x^{2}+\varepsilon W\left(\frac{x}{\varepsilon}\right),
$$

where $W$ is a one-periodic odd Lipschitz function with $\left\|W^{\prime}\right\|_{\infty}=1$. Up to addition of a constant is not restrictive to suppose that the average of $W$ is 0 . We check that the critical regime for the minimizing movements along $F_{\varepsilon}$ is $\varepsilon \sim \tau$. Indeed, if $\varepsilon<<\tau$ then from the estimate

$$
\left|F_{\varepsilon}(x)-\frac{1}{2} x^{2}\right| \leq \frac{\varepsilon}{2}
$$

we deduce that

$$
\frac{x_{k}-x_{k-1}}{\tau}=-x_{k}+O\left(\frac{\varepsilon}{\tau}\right)
$$

and hence that the limit minimizing movement satisfies $u^{\prime}=-u$, so that it corresponds to the minimizing movement of the limit $F_{0}(x)=\frac{1}{2} x^{2}$.

Conversely, if $\tau<<\varepsilon$ then it may be seen that for $\left|x_{0}\right| \leq 1$ the motion is pinned; i.e., the resulting minimizing movement is trivial $u(t)=x_{0}$ for all $t$. If $W \in C^{1}$ this is easily seen since in this case the stationary solutions, corresponding to $x$ satisfying

$$
x+W^{\prime}\left(\frac{x}{\varepsilon}\right)=0
$$

tend to be dense in the interval $[-1,1]$ as $\varepsilon \rightarrow 0$. Moreover, it is easily seen that in this regime the minimizing movement corresponds to the limit as $\varepsilon \rightarrow 0$ to the minimizing movements of $F_{\varepsilon}$ for $\varepsilon$ fixed; i.e., solutions $u_{\varepsilon}$ of the gradient flow

$$
u_{\varepsilon}^{\prime}=-u_{\varepsilon}-W^{\prime}\left(\frac{u_{\varepsilon}}{\varepsilon}\right) .
$$

Integrating between $t_{1}$ and $t_{2}$ we have

$$
\int_{u_{\varepsilon}\left(t_{1}\right)}^{u_{\varepsilon}\left(t_{2}\right)} \frac{1}{s+W^{\prime}(s / \varepsilon)} d s=t_{1}-t_{2} .
$$


By the uniform convergence $u_{\varepsilon} \rightarrow u$ we can pass to the limit, recalling that the integrand weakly converges to the function $1 / g$ defined by

$$
\frac{1}{g(s)}=\int_{0}^{1} \frac{1}{s+W^{\prime}(\sigma)} d \sigma,
$$

and obtain the equation

$$
u^{\prime}=-g(u)
$$

This equation corresponds to the minimizing movement for the even energy $\widetilde{F}_{0}$ given for $x \geq 0$

$$
\widetilde{F}_{0}(x)= \begin{cases}0 & \text { if } x \leq 1 \\ \int_{1}^{x} g(w) d w & \text { if } x \geq 1 .\end{cases}
$$

The plot of the derivatives of $F_{\varepsilon}, F_{0}$ and $\widetilde{F}_{0}$ is reproduced in Fig. 7.1

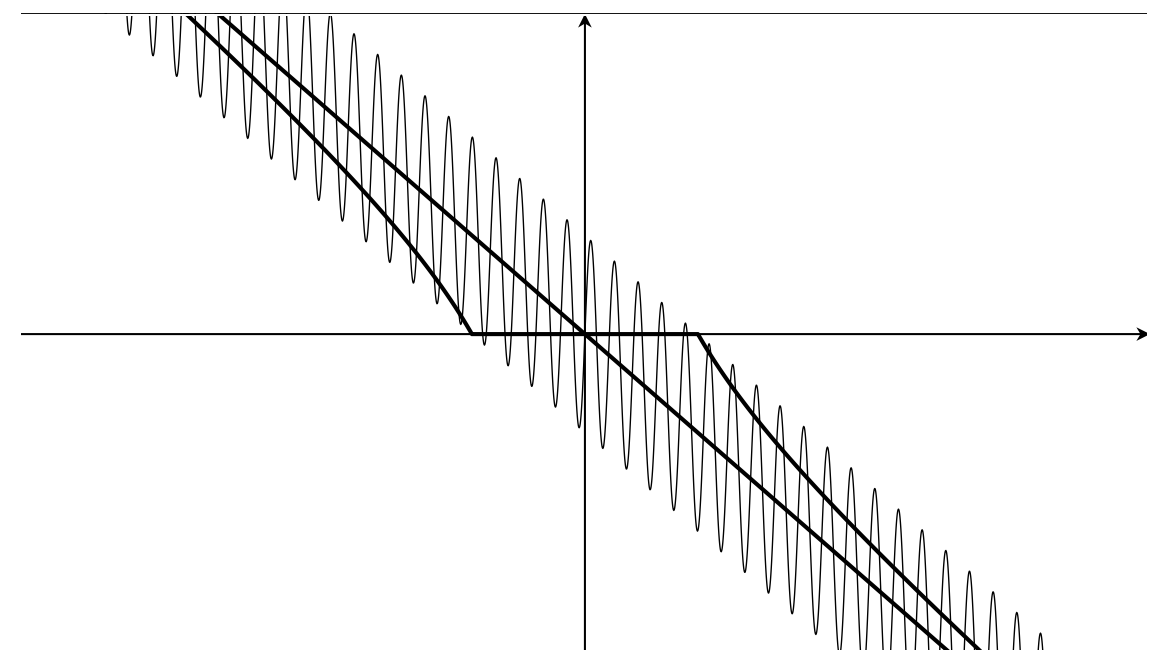

Figure 7.1: derivatives of $F_{\varepsilon}, F_{0}$ and $\widetilde{F}_{0}$

We can explicitly compute the minimizing movement for $\tau<<\varepsilon$ in the case $W(x)=$ $\left|x-\frac{1}{2}\right|-\frac{1}{4}$ for $0 \leq x \leq 1$. Here, the solutions with initial datum $x_{0}>1$ satisfy the equation

$$
u^{\prime}=\frac{1}{u}-u
$$

Integrating this limit equation we conclude that the minimizing movement along $F_{\varepsilon}$ correspond to that of the effecitve energy

$$
\widetilde{F}_{0}(x)=\left(\frac{1}{2} x^{2}-\log |x|-\frac{1}{2}\right)^{+} .
$$




\subsubsection{Relaxed evolution}

In Theorem 7.2.1 we have considered as usual by simplicity the $\Gamma$-convergence with respect to the topology in $X$. In this way we characterize the convergence of solutions to problems (7.4) to solutions of problems (7.5) in terms of the $\Gamma$-limit. This is the only argument where we have used the definition of $F$ in the proof of Theorem 7.2.1(i). We may consider the $\Gamma$-limits with respect to weaker topologies, for which we have coerciveness but the distance term is not a continuous perturbation. In analogy with what already observed for quasi static motions in Chapter 2 (see, e.g., Section 2.1.5) the proof of Theorem 7.2.1(i) can be repeated, upon defining a relaxed limit motion, where the minimizing movement for $F$ is replaced by the limit of $u^{\tau}$, defined by successive minimizing

$$
\min _{X} \mathcal{F}_{\tau}^{x_{k-1}}(x)
$$

where

$$
\mathcal{F}_{\tau}^{y}(x)=\Gamma-\lim _{\varepsilon \rightarrow 0}\left(F_{\varepsilon}(x)+\frac{1}{2 \tau}\|x-y\|^{2}\right) .
$$

The study of this more general minimizing movements is beyond the scope of these notes. We only give a simple example.

Example 7.2.5 Consider $X=L^{2}(0,1)$ and

$$
F_{\varepsilon}(u)=\int_{0}^{1} a\left(\frac{x}{\varepsilon}\right) u^{2} d x
$$

where $a$ is 1 -periodic and $0<\alpha \leq a(y) \leq \beta<+\infty$ for some constants $\alpha$ and $\beta$. Then $F_{\varepsilon}$ is equicoercive with respect to the weak- $L^{2}$ topology, and its limit is $\underline{a} \int_{0}^{1} u^{2} d x$ ( $\underline{a}$ the harmonic mean of $a$ ). However, the perturbations with the $L^{2}$-distance are not continuous, and the limits in (7.9) with respect to the weak topology are easily computed as

$$
\begin{aligned}
\mathcal{F}_{\tau}^{v}(u) & =\Gamma-\lim _{\varepsilon \rightarrow 0}\left(F_{\varepsilon}(u)+\frac{1}{2 \tau}\|u-v\|^{2}\right) \\
& =\Gamma-\lim _{\varepsilon \rightarrow 0} \int_{0}^{1}\left(\left(a\left(\frac{x}{\varepsilon}\right)+\frac{1}{2 \tau}\right) u^{2}+\frac{\left(v^{2}-2 u v\right)}{2 \tau}\right) d x \\
& =\int_{0}^{1}\left(\underline{a}_{\tau} u^{2}+\frac{\left(v^{2}-2 u v\right)}{2 \tau}\right) d x \\
& =\int_{0}^{1}\left(\underline{a}_{\tau}-\frac{1}{2 \tau}\right) u^{2} d x+\frac{1}{2 \tau}\|u-v\|^{2}
\end{aligned}
$$

where

$$
\underline{a}_{\tau}=\left(\int_{0}^{1} \frac{1}{\left(a(y)+\frac{1}{2 \tau}\right)} d y\right)^{-1}
$$


A series expansion argument easily yields that

$$
\begin{aligned}
\underline{a}_{\tau} & =\frac{1}{2 \tau}\left(\int_{0}^{1} \frac{1}{2 \tau a(y)+1} d y\right)^{-1} \\
& =\frac{1}{2 \tau}\left(\int_{0}^{1}\left(1-2 \tau a(y)+O\left(\tau^{2}\right)\right) d y\right)^{-1} \\
& =\frac{1}{2 \tau}\left(1+2 \tau \int_{0}^{1} a(y) d y+O\left(\tau^{2}\right)\right)=\frac{1}{2 \tau}+\bar{a}+O(\tau),
\end{aligned}
$$

where $\bar{a}$ is the arithmetic mean of $a$. We then obtain that the limit of $u^{\tau}$ coincides with the minimizing motion for $\widetilde{F}$ given by

$$
\widetilde{F}(u)=\bar{a} \int_{0}^{1} u^{2} d x .
$$

The same argument leading to an effective motion can be applied to varying distances as in the following example.

Example 7.2.6 We consider $X_{\varepsilon}=X=L^{2}(0,1)$ equipped with the distance $d_{\varepsilon}$ given by

$$
d_{\varepsilon}^{2}(u, v)=\int_{0}^{1} a\left(\frac{x}{\varepsilon}\right)|u-v|^{2} d x
$$

and $F_{\varepsilon}(u)=F(u)=\int_{0}^{1}\left|u^{\prime}\right|^{2} d x$. For fixed $v$ the square distances can be seen as functionals depending on $v$, weakly equicoercive in $L^{2}$ and $\Gamma$-converging to $\underline{a}\|u-v\|^{2}\left(\|u\|\right.$ the $L^{2}$-norm). Nevertheless, in this case the functionals $F_{\varepsilon}(u)+\frac{1}{2 \tau} d_{\varepsilon}^{2}(u, v)$ are coercive with respect to the strong $L^{2}$-norm and $\Gamma$-converge to $F(u)+\frac{1}{2 \tau} \bar{a}\|u-v\|^{2}$. As a conclusion, the minimizing movement coincide with the minimizing movement for $F$ with respect to the norm $\sqrt{\bar{a}}\|u\|$ or, equivalently, with the minimizing movement for $\frac{1}{\bar{a}} F$ with respect to the $L^{2}$-norm.

\subsection{An example: 'overdamped dynamics' of Lennard-Jones interactions}

We now give an example of a sequence of non-convex energies which commute with the minimizing movement procedure.

Let $J$ be as in Section 3.4 and $\frac{1}{\varepsilon}=N \in \mathbb{N}$. We consider the energies

$$
F_{\varepsilon}(u)=\sum_{i=1}^{N} J\left(\frac{u_{i}-u_{i-1}}{\sqrt{\varepsilon}}\right)
$$


with the periodic boundary condition $u_{N}=u_{0}$. As proved in Section 3.4, after identification of $u$ with a piecewise-constant function on $[0,1]$, these energies $\Gamma$-converge to the energy

$$
F(u)=\int_{0}^{1}\left|u^{\prime}\right|^{2} d t+\#(S(u) \cap[0,1)), \quad u^{+}>u^{-}
$$

defined on piecewise- $H^{1}$ functions, in this case extended 1-periodically on the whole real line.

In this section we apply the minimizing movements scheme to $F_{\varepsilon}$ as a sequence of functionals in $L^{2}(0,1)$. In order to have initial data $u_{0}^{\varepsilon}$ with equibounded energy, we may suppose that these are the discretization of a single piecewise- $H^{1}$ function $u_{0}$ (with a slight abuse of notation we will continue to denote all these discrete functions by $u_{0}$ ).

With fixed $\varepsilon$ and $\tau$, the time-discretization scheme consists in defining recursively $u^{k}$ as a minimizer of

$$
u \mapsto \sum_{i=1}^{N} J\left(\frac{u_{i}-u_{i-1}}{\sqrt{\varepsilon}}\right)+\frac{1}{2 \tau} \sum_{i=1}^{N} \varepsilon\left|u_{i}-u_{i}^{k-1}\right|^{2} .
$$

By Proposition 7.1.2, upon extraction of a subsequence, the functions $u^{\tau}(t)=u_{\lfloor t / \tau\rfloor}$ converge uniformly in $L^{2}$ to a function $u \in C^{1 / 2}\left([0,+\infty) ; L^{2}(0,1)\right)$. Moreover, since we have $F(u(t)) \leq F\left(u_{0}\right)<+\infty, u(t)$ is a piecewise- $H^{1}$ function for all $t$.

We now describe the motion of the limit $u$. For the sake of simplicity we suppose that $u_{0}$ is a piecewise-Lipschitz function and that $S\left(u_{0}\right) \cap\{\varepsilon i: i \in\{1, \ldots, N\}\}=\emptyset$ (so that we do not have any ambiguity in the definition of the interpolations of $u_{0}$ )..

We first write down the Euler-Lagrange equations for $u^{k}$, which amount to a simple $N$-dimensional system of equations obtained by deriving (7.10) with respect to $u_{i}$

$$
\frac{1}{\sqrt{\varepsilon}}\left(J^{\prime}\left(\frac{u_{i}^{k}-u_{i-1}^{k}}{\sqrt{\varepsilon}}\right)-J^{\prime}\left(\frac{u_{i+1}^{k}-u_{i}^{k}}{\sqrt{\varepsilon}}\right)\right)+\frac{\varepsilon}{\tau}\left(u_{i}^{k}-u_{i}^{k-1}\right)=0 .
$$

- With fixed $i \in\{1, \ldots, N\}$ let $v_{k}$ be defined by

$$
v_{k}=\frac{u_{i}^{k}-u_{i-1}^{k}}{\varepsilon} .
$$

For simplicity of notation we set

$$
J_{\varepsilon}(w)=\frac{1}{\varepsilon} J(\sqrt{\varepsilon} w)
$$

By (7.11) and the corresponding equation for $i-1$, which can be rewritten as

$$
J_{\varepsilon}^{\prime}\left(\frac{u_{i-1}^{k}-u_{i-2}^{k}}{\varepsilon}\right)-J_{\varepsilon}^{\prime}\left(\frac{u_{i}^{k}-u_{i-1}^{k}}{\varepsilon}\right)+\frac{\varepsilon}{\tau}\left(u_{i-1}^{k}-u_{i-1}^{k-1}\right)=0 .
$$


we have

$$
\begin{aligned}
\frac{v_{k}-v_{k-1}}{\tau}= & \frac{1}{\tau}\left(\frac{u_{i}^{k}-u_{i-1}^{k}}{\varepsilon}-\frac{u_{i}^{k-1}-u_{i-1}^{k-1}}{\varepsilon}\right) \\
= & \frac{1}{\varepsilon}\left(\frac{u_{i}^{k}-u_{i}^{k-1}}{\tau}-\frac{u_{i-1}^{k}-u_{i-1}^{k-1}}{\tau}\right) \\
= & \frac{1}{\varepsilon^{2}}\left(\left(J_{\varepsilon}^{\prime}\left(\frac{u_{i-1}^{k}-u_{i-2}^{k}}{\varepsilon}\right)-J_{\varepsilon}^{\prime}\left(\frac{u_{i}^{k}-u_{i-1}^{k}}{\varepsilon}\right)\right)\right. \\
& \left.-\left(J_{\varepsilon}^{\prime}\left(\frac{u_{i}^{k}-u_{i-1}^{k}}{\varepsilon}\right)-J_{\varepsilon}^{\prime}\left(\frac{u_{i+1}^{k}-u_{i}^{k}}{\varepsilon}\right)\right)\right)
\end{aligned}
$$

so that

$$
\begin{aligned}
\frac{v_{k}-v_{k-1}}{\tau}-\frac{2}{\varepsilon^{2}} J_{\varepsilon}^{\prime}\left(v_{k}\right) & =-\frac{1}{\varepsilon^{2}}\left(J_{\varepsilon}^{\prime}\left(\frac{u_{i-1}^{k}-u_{i-2}^{k}}{\varepsilon}\right)+J_{\varepsilon}^{\prime}\left(\frac{u_{i+1}^{k}-u_{i}^{k}}{\varepsilon}\right)\right) \\
& \geq-\frac{2}{\varepsilon^{2}} J_{\varepsilon}^{\prime}\left(\frac{w_{0}}{\sqrt{\varepsilon}}\right) .
\end{aligned}
$$

We recall that we denote by $w_{0}$ the maximum point of $J^{\prime}$.

We can interpret (7.12) as an inequality for the difference system

$$
\frac{v_{k}-v_{k-1}}{\eta}-2 J_{\varepsilon}^{\prime}\left(v_{k}\right) \geq-2 J_{\varepsilon}^{\prime}\left(\frac{w_{0}}{\sqrt{\varepsilon}}\right),
$$

where $\eta=\tau / \varepsilon^{2}$ is interpreted as a discretization step. Note that $v_{k}=w_{0} / \sqrt{\varepsilon}$ for all $k$ is a stationary solution of the equation

$$
\frac{v_{k}-v_{k-1}}{\eta}-2 J_{\varepsilon}^{\prime}\left(v_{k}\right)=-2 J_{\varepsilon}^{\prime}\left(\frac{w_{0}}{\sqrt{\varepsilon}}\right)
$$

and that $J_{\varepsilon}^{\prime}$ are equi-Lipschitz functions on $[0,+\infty)$. If $\eta<<1$ this implies that if $v_{k_{0}} \leq$ $w_{0} / \sqrt{\varepsilon}$ for some $k_{0}$ then

$$
v_{k} \leq \frac{w_{0}}{\sqrt{\varepsilon}} \quad \text { for } k \geq k_{0},
$$

or, equivalently, that if $\tau<<\varepsilon^{2}$ the set

$$
S_{\varepsilon}^{k}=\left\{i \in\{1, \ldots, N\}: \frac{u_{i}^{k}-u_{i-1}^{k}}{\varepsilon} \geq \frac{w_{0}}{\sqrt{\varepsilon}}\right\}
$$

is decreasing with $k$. By our assumption on $u_{0}$, for $\varepsilon$ small enough we then have

$$
S_{\varepsilon}^{0}=\left\{i \in\{1, \ldots, N\}:[\varepsilon(i-1), \varepsilon i] \cap S\left(u_{0}\right) \neq \emptyset\right\},
$$


so that, passing to the limit

$$
S(u(t)) \subseteq S\left(u_{0}\right) \quad \text { for all } t \geq 0 .
$$

- Taking into account that we may define

$$
u^{\tau}(t, x)=u_{\lfloor x / \varepsilon\rfloor}^{\lfloor t / \tau\rfloor}
$$

we may choose functions $\phi \in C_{0}^{\infty}(0, T)$ and $\psi \in C_{0}^{\infty}\left(x_{1}, x_{2}\right)$, with $\left(x_{1}, x_{2}\right) \cap S\left(u_{0}\right)=\emptyset$, and obtain from (7.11)

$$
\begin{aligned}
& \int_{0}^{T} \int_{x_{1}}^{x_{2}} u^{\tau}(t, x)\left(\frac{\phi(t)-\phi(t+\tau)}{\tau}\right) \psi(x) d x d t \\
= & -\int_{0}^{T} \int_{x_{1}}^{x_{2}}\left(\frac{1}{\sqrt{\varepsilon}} J^{\prime}\left(\sqrt{\varepsilon} \frac{u^{\tau}(t, x)-u^{\tau}(t, x-\varepsilon)}{\varepsilon}\right)\right) \phi(t)\left(\frac{\psi(x)-\psi(x+\varepsilon)}{\varepsilon}\right) d x d t .
\end{aligned}
$$

Taking into account that

$$
\lim _{\varepsilon \rightarrow 0} \frac{1}{\sqrt{\varepsilon}} J^{\prime}(\sqrt{\varepsilon} w)=2 w
$$

we can pass to the limit and obtain that

$$
-\int_{0}^{T} \int_{x_{1}}^{x_{2}} u(t, x) \phi^{\prime}(t) \psi(x) d x d t=\int_{0}^{T} \int_{x_{1}}^{x_{2}} 2 \frac{\partial u}{\partial x} \phi(t) \psi^{\prime}(x) d x d t
$$

i.e., that

$$
\frac{\partial u}{\partial t}=-2 \frac{\partial^{2} u}{\partial x^{2}}
$$

in the sense of distributions (and hence also classically) in $(0, T) \times\left(x_{1}, x_{2}\right)$. By the arbitrariness of the interval $\left(x_{1}, x_{2}\right)$ we have that equation (7.14) is satisfied for $x$ in $(0,1) \backslash S\left(u_{0}\right)$.

- We now derive boundary conditions on $S(u(t))$. Let $i_{0}+1$ belong to $S_{\varepsilon}^{0}$, and suppose that $u^{+}(t, x)-u^{-}(t, x) \geq c>0$. Then we have

$$
\lim _{\tau \rightarrow 0} \frac{1}{\sqrt{\varepsilon}} J^{\prime}\left(\frac{u_{i_{0}}^{\lfloor t / \tau\rfloor}-u_{i_{0}-1}^{\lfloor t / \tau\rfloor}}{\sqrt{\varepsilon}}\right)=0 .
$$

If $i<i_{0}$, from (7.11) it follows, after summing up the indices from $i$ to $i_{0}$, that

$$
\sum_{j=i}^{i_{0}} \frac{\varepsilon}{\tau}\left(u_{j}^{k}-u_{j}^{k-1}\right)=-\frac{1}{\sqrt{\varepsilon}} J^{\prime}\left(\frac{u_{i}^{k}-u_{i-1}^{k}}{\sqrt{\varepsilon}}\right) .
$$

We may choose $i=i_{\varepsilon}$ such that $\varepsilon i_{\varepsilon} \rightarrow \bar{x}$ and we may deduce from (7.15) that

$$
\int_{\bar{x}}^{x_{0}} \frac{\partial u}{\partial t} d x=-2 \frac{\partial u}{\partial x}(\bar{x})
$$


where $x_{0} \in S(u(t))$ is the limit of $\varepsilon i_{0}$. Letting $\bar{x} \rightarrow x_{0}^{-}$we obtain

$$
\frac{\partial u}{\partial x}\left(x_{0}^{-}\right)=0
$$

Similarly we obtain the homogeneous Neumann condition at $x_{0}^{+}$.

Summarizing, the minimizing movement of the scaled Lennard-Jones energies $F_{\varepsilon}$ from a piecewise- $H^{1}$ function consists in a piecewise- $H^{1}$ motion, following the heat equation on $(0,1) \backslash S\left(u_{0}\right)$, with homogeneous Neumann boundary conditions on $S\left(u_{0}\right)$ (as long as $u(t)$ has a discontinuity at the corresponding point of $\left.S\left(u_{0}\right)\right)$.

Note that for $\varepsilon \rightarrow 0$ sufficiently fast Theorem 7.2.1 directly ensures that the minimizing movement along $F_{\varepsilon}$ coincides with the minimizing movement for the functional $F$. The computation above shows that this holds also for $\tau<<\varepsilon^{2}$ (i.e., $\varepsilon \rightarrow 0$ 'sufficiently slow'), which then must be regarded as a technical condition.

\subsection{Homogenization of minimizing movements}

We now examine minimizing movements along oscillating sequences (with many local minima), treating two model cases in the real line.

\subsubsection{Minimizing movements for piecewise-constant energies}

We apply the minimizing-movement scheme to the functions

$$
F_{\varepsilon}(x)=-\left\lfloor\frac{x}{\varepsilon}\right\rfloor \varepsilon
$$

converging to $F(x)=-x$ (see Fig. 7.2). This is a prototype of a function with many local

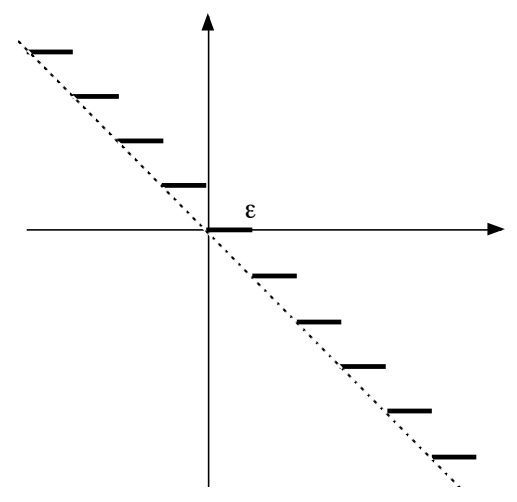

Figure 7.2: the function $F_{\varepsilon}$ 
minimizers (actually, in this case all points are local minimizers) converging to a function with few local minimizers (actually, none).

Note that, with fixed $\varepsilon$, for any initial datum $x_{0}$ the minimizing movement for $F_{\varepsilon}$ is trivial: $u(t)=x_{0}$, since all points are local minimizers. Conversely the corresponding minimizing movement for the limit is $u(t)=x_{0}+t$.

We now fix an initial datum $x_{0}$, the space scale $\varepsilon$ and the time scale $\tau$, and examine the successive-minimization scheme from $x_{0}$. Note that it is not restrictive to suppose that $0 \leq x_{0}<1$ up to a translation in $\varepsilon \mathbb{Z}$.

The first minimization, giving $x_{1}$ is

$$
\min \left\{F_{\varepsilon}(x)+\frac{1}{2 \tau}\left(x-x_{0}\right)^{2}\right\} .
$$

The function to minimize is pictured in Figure 7.3 in normalized coordinates $(\varepsilon=1)$; note that it equals $-x+\frac{1}{2 \tau}\left(x-x_{0}\right)^{2}$ if $x \in \varepsilon \mathbb{Z}$.

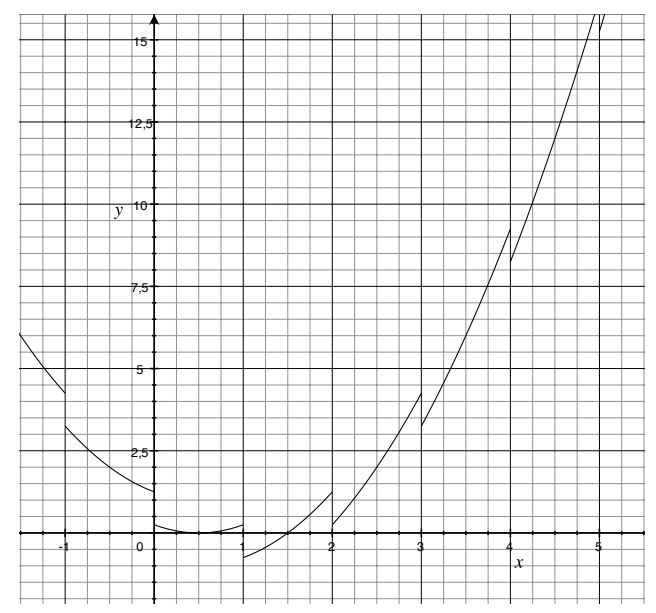

Figure 7.3: the function in the minimization problem (7.16)

Except for some exceptional cases that we deal separately below, we have two possibilities:

(i) if $\frac{\tau}{\varepsilon}<\frac{1}{2}$ then the motion is trivial. The value $1 / 2$ is the pinning threshold.

If we set $x_{0}=s \varepsilon$ with $0 \leq s<1$. Then we have two sub-cases: i.e.,

(a) the minimizer $x_{1}$ belongs to $[0, \varepsilon)$. This occurs exactly if $F_{\varepsilon}(\varepsilon)+\frac{1}{2 \tau}\left(\varepsilon-x_{0}\right)^{2}>0$;

$$
\tau<\frac{(s-1)^{2} \varepsilon}{2} .
$$

In this case the only minimizer is still $x_{0}$. This implies that we have $x_{k}=x_{0}$ for all $k$. Otherwise, we have that $x_{1}=\varepsilon$. This implies that, up to a translation we are in the case $x_{0}=0$ with $s=0$, and (7.17) holds since $\tau<\frac{\varepsilon}{2}$. Hence, $x_{k}=x_{1}$ for all $k \geq 1$; 
(ii) if $\frac{\tau}{\varepsilon}>\frac{1}{2}$ then for $\varepsilon$ small the minimum is taken on $\varepsilon \mathbb{Z}$. So that again we may suppose that $x_{0}=0$.

Note that we are leaving out for now the case when $x_{0}=0$ and $\frac{\tau}{\varepsilon}=\frac{1}{2}$. In this case we have a double choice for the minimizer; such situations will be examined separately.

If $x_{0}=0$ then $x_{1}$ is computed by solving

$$
\min \left\{F_{\varepsilon}(x)+\frac{1}{2 \tau} x^{2}: x \in \varepsilon \mathbb{Z}\right\}
$$

and is characterized by

We then have

$$
x_{1}-\frac{1}{2} \varepsilon \leq \tau \leq x_{1}+\frac{1}{2} \varepsilon
$$

$$
x_{1}=\left\lfloor\frac{\tau}{\varepsilon}+\frac{1}{2}\right\rfloor \varepsilon \quad \text { if } \frac{\tau}{\varepsilon}+\frac{1}{2} \notin \mathbb{Z}
$$

(note again that we have two solutions for $\frac{\tau}{\varepsilon}+\frac{1}{2} \in \mathbb{Z}$, and we examine this case separately). The same computation is repeated at each $k$ giving

$$
\frac{x_{k}-x_{k-1}}{\tau}=\left\lfloor\frac{\tau}{\varepsilon}+\frac{1}{2}\right\rfloor \frac{\varepsilon}{\tau} .
$$

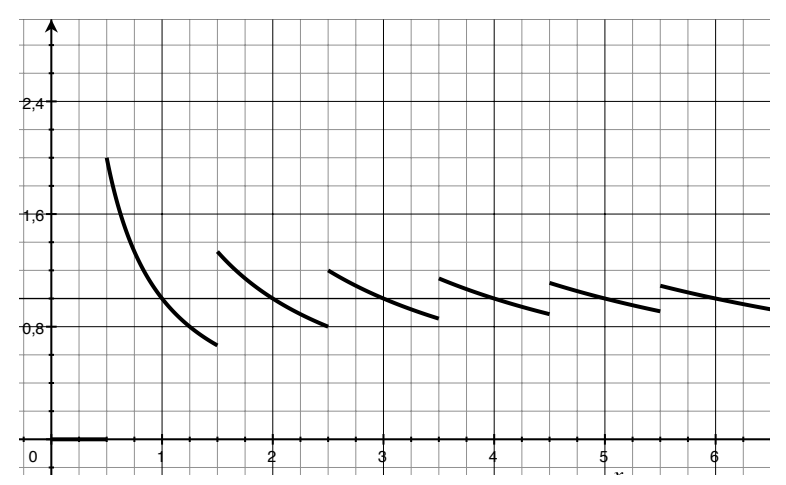

Figure 7.4: the velocity $v$ in terms of $w$

We can now choose $\tau$ and $\varepsilon$ tending to 0 simultaneously and pass to the limit. The behaviour of the limit minimizing movements is governed by the quantity

$$
w=\lim _{\varepsilon \rightarrow 0} \frac{\tau}{\varepsilon},
$$

which we may suppose exists up to subsequences. If $w+\frac{1}{2} \notin \mathbb{Z}$ then the minimizing movement along $F_{\varepsilon}$ from $x_{0}$ is uniquely defined by

$$
u(t)=x_{0}+v t, \text { with } v=\left\lfloor w+\frac{1}{2}\right\rfloor \frac{1}{w},
$$


so that the whole sequence converges if the limit in (7.19) exists. Note that

- (pinning) we have $v=0$ exactly when $\frac{\tau}{\varepsilon}<\frac{1}{2}$ for $\varepsilon$ small. In particular this holds for $\tau<<\varepsilon$ (i.e., for $w=0$ );

- (limit motion for slow times) if $\varepsilon<<\tau$ then the motion coincides with the gradient flow of the limit, with velocity 1 ;

- (discontinuous dependence of the velocity) the velocity is a discontinuous function of $w$ at points of $\frac{1}{2}+\mathbb{Z}$. Note moreover that it may be actually greater than the limit velocity 1. The graph of $v$ is pictured in Figure 7.4

- (non-uniqueness at $w \in \frac{1}{2}+\mathbb{Z}$ ) in these exceptional cases we may have either of the two velocities $1+\frac{1}{2 w}$ or $1-\frac{1}{2 w}$ in the cases $\frac{\varepsilon}{\tau}+\frac{1}{2}>w$ or $\frac{\varepsilon}{\tau}+\frac{1}{2}<w$ for all $\varepsilon$ small respectively, but we may also have any $u(t)$ with

$$
1-\frac{1}{2 w} \leq u^{\prime}(t) \leq 1+\frac{1}{2 w}
$$

if we have precisely $\frac{\varepsilon}{\tau}+\frac{1}{2}=w$ for all $\varepsilon$ small, since in this case at every time step we may choose any of the two minimizers giving the extremal velocities, and then obtain any such $u^{\prime}$ as a weak limit of piecewise constant functions taking only those two values. Note therefore that in this case the limit is not determined only by $w$, and in particular it may depend on the subsequence even if the limit (7.19) exists.

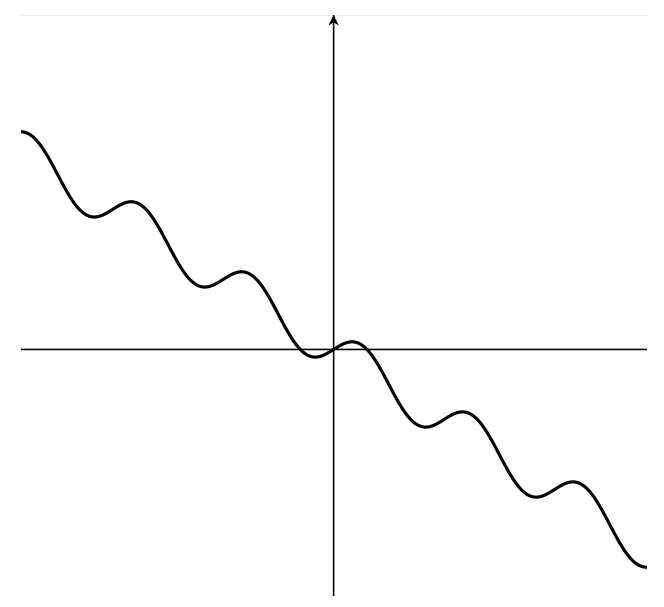

Figure 7.5: other potentials giving the same homogenization pattern

We remark that the functions $F_{\varepsilon}$ above can be substituted by functions with isolated local minimizers; e.g. by taking $(\alpha>0)$

$$
F_{\varepsilon}(x)=-\left\lfloor\frac{x}{\varepsilon}\right\rfloor \varepsilon+\alpha\left(x-\left\lfloor\frac{x}{\varepsilon}\right\rfloor \varepsilon\right),
$$


with isolated local minimizers at $\varepsilon \mathbb{Z}$ (for which the computations run exactly as above), or

$$
F_{\varepsilon}(x)=-x+(1+\alpha) \varepsilon \sin \left(\frac{x}{\varepsilon}\right) .
$$

Note that the presence of an energy barrier between local minimizers does not influence the velocity of the final minimizing movement, that can always be larger than 1 (the velocity as $\varepsilon<<\tau$ ).

We also remark that the same result can be obtained by a 'discretization' of $F$; i.e., taking

$$
F_{\varepsilon}(x)= \begin{cases}-x & \text { if } x \in \varepsilon \mathbb{Z} \\ +\infty & \text { otherwise }\end{cases}
$$

\subsubsection{A heterogeneous case}

We briefly examine a variation of the previous example introducing a heterogeneity parameter $1 \leq \lambda \leq 2$ and defining

$$
F^{\lambda}(x)= \begin{cases}-2\left\lfloor\frac{x}{2}\right\rfloor & \text { if } 2\left\lfloor\frac{x}{2}\right\rfloor \leq x<2\left\lfloor\frac{x}{2}\right\rfloor+\lambda \\ -2\left\lfloor\frac{x}{2}\right\rfloor-\lambda & \text { if } 2\left\lfloor\frac{x}{2}\right\rfloor+\lambda \leq x<2\left\lfloor\frac{x}{2}\right\rfloor+1\end{cases}
$$

If $\lambda=1$ we are in the previous situation; for general $\lambda$ the function $F^{\lambda}$ is pictured in Fig. 7.6.

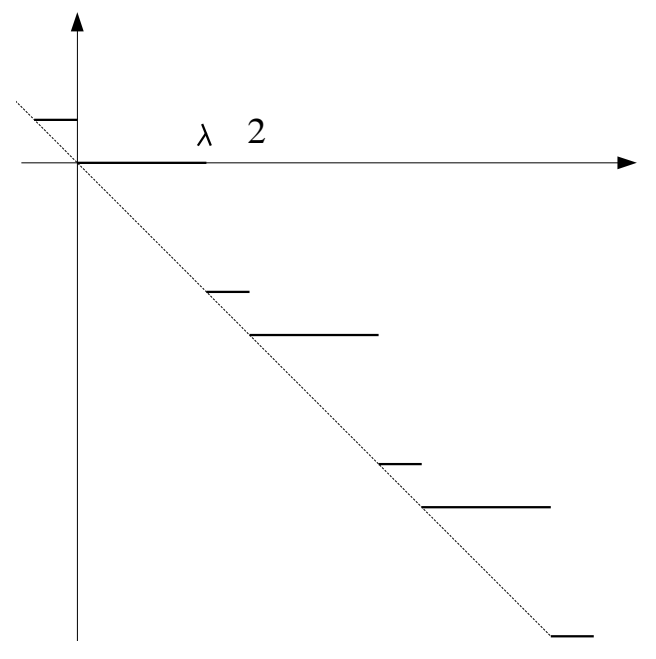

Figure 7.6: the function $F^{\lambda}$ 
We apply the minimizing-movement scheme to the functions

$$
F_{\varepsilon}(x)=F_{\varepsilon}^{\lambda}(x)=\varepsilon F^{\lambda}\left(\frac{x}{\varepsilon}\right)
$$

Arguing as above, we can reduce to the two cases

$$
\text { (a) } x_{k} \in 2 \varepsilon \mathbb{Z}, \quad \text { or } \quad \text { (b) } x_{k} \in 2 \varepsilon \mathbb{Z}+\varepsilon \lambda \text {. }
$$

Taking into account that $x_{k+1}$ is determined as the point in $2 \varepsilon \mathbb{Z} \cup(2 \varepsilon \mathbb{Z}+\varepsilon \lambda)$ closer to $\tau$ (as above, we only consider the cases when we have a unique solution to the minimum problems in the iterated procedure), we can characterize it as follows.

In case (a) we have the two sub cases:

$\left(a_{1}\right)$ if we have

$$
2 n<\frac{\tau}{\varepsilon}-\frac{\lambda}{2}<2 n+1
$$

for some $n \in \mathbb{N}$ then

$$
x_{k+1}=x_{k}+(2 n+\lambda) \varepsilon .
$$

In particular $x_{k+1} \in 2 \varepsilon \mathbb{Z}+\varepsilon \lambda$;

$\left(a_{2}\right)$ if we have

$$
2 n-1<\frac{\tau}{\varepsilon}-\frac{\lambda}{2}<2 n
$$

for some $n \in \mathbb{N}$ then

$$
x_{k+1}=x_{k}+2 n \varepsilon .
$$

In particular $x_{k+1} \in 2 \varepsilon \mathbb{Z}$. Note that $x_{k+1}=x_{k}$ (pinning) if $\frac{\tau}{\varepsilon}<\frac{\lambda}{2}$.

In case (b) we have the two sub cases:

$\left(b_{1}\right)$ if we have

$$
2 n<\frac{\tau}{\varepsilon}+\frac{\lambda}{2}<2 n+1
$$

for some $n \in \mathbb{N}$ then

$$
x_{k+1}=x_{k}+2 n \varepsilon .
$$

In particular $x_{k+1} \in 2 \varepsilon \mathbb{Z}+\varepsilon \lambda$. Note that $x_{k+1}=x_{k}$ (pinning) if $\frac{\tau}{\varepsilon}<1-\frac{\lambda}{2}$, which is implied by the pinning condition in $\left(\mathrm{a}_{2}\right)$;

$\left(b_{2}\right)$ if we have

$$
2 n-1<\frac{\tau}{\varepsilon}+\frac{\lambda}{2}<2 n
$$

for some $n \in \mathbb{N}$ then

$$
x_{k+1}=x_{k}+2 n \varepsilon-\varepsilon \lambda .
$$

In particular $x_{k+1} \in 2 \varepsilon \mathbb{Z}$. 
Eventually, we have the two cases:

(1) when

$$
\left|\frac{\tau}{\varepsilon}-2 n\right|<\frac{\lambda}{2}
$$

for some $n \in \mathbb{N}$ then, after possibly one iteration, we are either in the case $\left(\mathrm{a}_{2}\right)$ or $\left(\mathrm{b}_{1}\right)$. Hence, either $x_{k} \in 2 \varepsilon \mathbb{Z}$ or $x_{k} \in 2 \varepsilon \mathbb{Z}+\varepsilon \lambda$ for all $k$. The velocity in this case is then

$$
\frac{x_{k+1}-x_{k}}{\tau}=2 n \frac{\varepsilon}{\tau}
$$

(2) when

$$
\left|\frac{\tau}{\varepsilon}-(2 n+1)\right|<1-\frac{\lambda}{2}
$$

for some $n \in \mathbb{N}$ then we are alternately in case $\left(\mathrm{a}_{1}\right)$ or $\left(\mathrm{b}_{2}\right)$. In this case we have an

- averaged velocity: the speed of the orbit $\left\{x_{k}\right\}$ oscillates between two values with a mean speed given by

$$
\frac{x_{k+2}-x_{k}}{2 \tau}=\frac{2 n \varepsilon+\lambda \varepsilon}{2 \tau}+\frac{2(n+1) \varepsilon-\lambda \varepsilon}{2 \tau}=(2 n+1) \frac{\varepsilon}{\tau} .
$$

This is an additional feature with respect to the previous example.

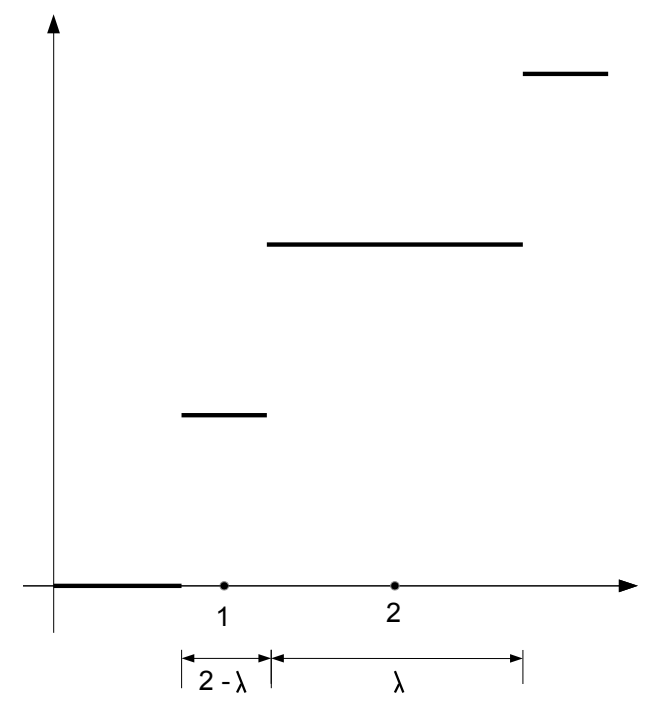

Figure 7.7: The function $f$ describing the effective velocity 
Summarizing, if we define $w$ as in (7.19) then (taking into account only the cases with a unique limit) the minimizing movement along the sequence $F_{\varepsilon}$ with initial datum $x_{0}$ is given by $x(t)=x_{0}+v t$ with $v=f(w) \frac{1}{w}$, and $f$ is given by

$$
f(w)= \begin{cases}2 n & \text { if }|w-2 n| \leq \frac{\lambda}{2}, n \in \mathbb{N} \\ 2 n+1 & \text { if }|w-(2 n+1)|<1-\frac{\lambda}{2}, n \in \mathbb{N}\end{cases}
$$

(see Fig. 7.7). Note that the pinning threshold is now $\lambda / 2$.

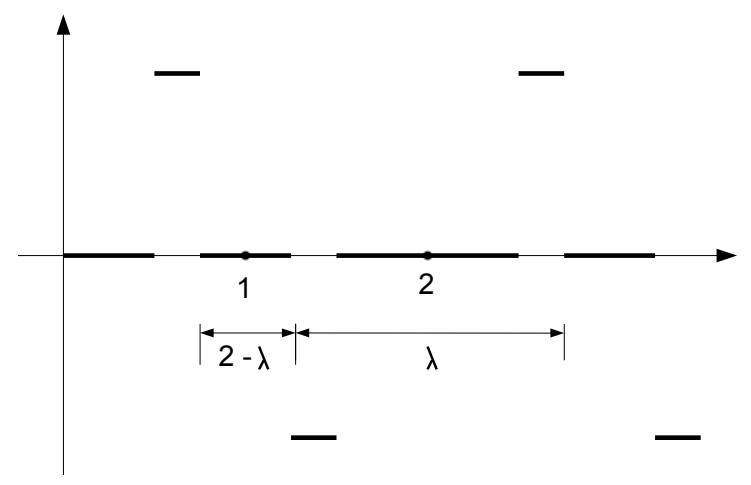

Figure 7.8: comparison with the homogeneous case

We can compare this minimizing movement with the one given in (7.20) by examining the graph of $w \mapsto\lfloor w+1 / 2\rfloor-f(w)$ in Fig. 7.8. For $2 n+1 / 2<w<2 n+\lambda / 2$ the new minimizing movement is slower, while for $2 n+2-\lambda / 2<w<2 n+2-1 / 2$ it is faster.

\subsubsection{A proposal for some random models}

From the heterogeneous example above we may derive two possible random models, of which we may then study the corresponding minimizing movement. We only give a heuristic proposal, which can then be correctly formalized by introducing suitable random variables.

1. Random environment. Let $\lambda \in(1 / 2,1)$ and $p \in[0,1]$. We consider a random array of points $\left\{x_{i}^{\omega}\right\}$ in $\mathbb{R}$ such that, e.g.,

$$
x_{i}^{\omega}-x_{i-1}^{\omega}= \begin{cases}\lambda & \text { with probability } p \\ 2-\lambda & \text { with probability } 1-p .\end{cases}
$$

With fixed $\omega$ we may consider the minimizing movement related to

$$
F_{\varepsilon}^{\omega}(x)= \begin{cases}-x & \text { if } x \in\left\{\varepsilon x_{i}^{\omega}: i \in \mathbb{Z}\right\} \\ +\infty & \text { otherwise, }\end{cases}
$$


or equivalently (as in the definition (7.21)

$$
F_{\varepsilon}^{\omega}(x)=-\varepsilon x_{i}^{\omega} \text { if } x \in\left[\varepsilon x_{i}^{\omega}, \varepsilon x_{i+1}^{\omega}\right), i \in \mathbb{Z} .
$$

In the case $p=0$ or $p=1$ we almost surely have a homogeneous environment as in Section 7.4.1. For $p=1 / 2$ we have a random version of the heterogeneous model of Section 7.4.2. Note that is this case for all $p \in(0,1)$ the pinning threshold for the ratio $\tau / \varepsilon$ is almost surely $\lambda / 2$, since below that value, the motion will be pinned at the first index $i$ with $x_{i}^{\omega}-x_{i-1}^{\omega}=\lambda$; i.e., almost surely after a finite number of steps. For $\tau / \varepsilon=\lambda / 2$ and $\lambda<2 / 3$ (with this condition we always move of one index) then the (maximal) velocity after pinning is $v=\lambda p+(1-p)$ (for $\lambda>2 / 3$ the computation of the velocity involves the probability of $m$-consecutive points $x_{i}^{\omega}$ at distance $\left.2-\lambda\right)$;

2. Random movements. Let $\lambda \in(1 / 2,1)$ and $p \in[0,1]$. Contrary to the model above, at every time step $k$ we may make a random choice of points $\left\{x_{i}^{\omega_{k}}\right\}$ satisfying $(7.23)$ such that $x_{k}^{\varepsilon} \in\left\{x_{i}^{\omega_{k}}\right\}$; i.e., this choice now represents the random possibility of motion of the point itself (and not a characteristic of the medium). Note that in this case for

$p \in(0,1)$ the pinning threshold for the ratio $\tau / \varepsilon$ is almost surely the lower value $1-\frac{\lambda}{2}$, and the (maximal) velocity after pinning is $v=(2-\lambda)(1-p)$.

\subsection{Time-dependent minimizing movements}

Following the arguments of Section 6.2 we can define a minimizing movement along a timedependent sequence of energies $F_{\varepsilon}(x, t)$, upon some technical assumptions as in (6.10). In this case we fix a sequence of initial data $x_{0}^{\varepsilon}$ and $\tau=\tau_{\varepsilon} \rightarrow 0$, and define recursively $x_{k}^{\varepsilon}$ as minimizing

$$
\min \left\{F_{\varepsilon}(x, k \tau)+\frac{1}{2 \tau}\left\|x-x_{k-1}^{\varepsilon}\right\|^{2}\right\} .
$$

A minimizing movement is then any limit $u$ of $u^{\varepsilon}$ defined $u^{\varepsilon}(t)=x_{\lfloor t / \tau\rfloor}$.

We only give a simple one-dimensional example with a time-dependent forcing term.

Example 7.5.1 We consider

$$
F_{\varepsilon}(x, t)=\varepsilon W\left(\frac{x}{\varepsilon}\right)-t x
$$

with $W$ as in Example 7.2.4. Similarly to that example we can check that $\varepsilon \sim \tau$ is the critical case, and we can explicitly describe the minimizing movement in the extreme cases:

- $(\varepsilon<<\tau)$ the minimizing movement is that corresponding to $F_{0}(x, t)=-t u$; i.e., to the equation $u^{\prime}=t$;

- $(\tau<<\varepsilon)$ the minimizing movement is that corresponding to the function

$$
\widetilde{F}_{0}(t, x)= \begin{cases}0 & \text { if } t \leq 1 \\ g(t) u & \text { if } t \geq 1\end{cases}
$$


where $g$ is now defined by

$$
\frac{1}{g(t)}=\int_{0}^{1} \frac{1}{W^{\prime}(\sigma)-t} d \sigma
$$

\subsection{References to Chapter 7}

The definition of minimizing movement along a sequence of functionals formalizes a natural extension to the notion of minimizing movement, and follows the definition given in the paper by Braides, Gelli and Novaga [14].

The energies in Examples 7.2.4 and 7.5.1 have been taken as a prototype to model plastic phenomena by Puglisi and Truskinovsky [40]. More recently that example has been recast in the framework of quasi static motion in the papers by Mielke and Truskinovsky $[39,35]$.

The example of the minimizing movement for Lennard Jones interactions is original, and is part of ongoing work with A. Defranceschi and E. Vitali. It is close in spirit to a semi-discrete approach (i.e., the study of the limit of the gradient flows for the discrete energies) by Gobbino [29]. 


\section{Chapter 8}

\section{Geometric minimizing movements}

We now examine some minimizing movements describing the motion of sets. Such a motion can be framed in the setting of the previous chapter after identification of a set $A$ with its characteristic function $u=\chi_{A}$. The energies we are going to consider are of perimeter type; i.e., with

$$
F(A)=\mathcal{H}^{n-1}(\partial A)
$$

as a prototype in the notation of the previous chapter.

\subsection{Motion by mean curvature}

The prototype of a geometric motion is motion by mean curvature; i.e., a family of sets $A(t)$ whose boundary moves in the normal direction with velocity proportional to its curvature (inwards in convex regions and outwards in concave regions). In the simplest case of initial datum a ball $A(0)=A_{0}=B_{R_{0}}(0)$ in $\mathbb{R}^{2}$ the motion is given by concentric balls with radii satisfying

$$
\left\{\begin{array}{l}
R^{\prime}=-\frac{c}{R} \\
R(0)=R_{0}
\end{array}\right.
$$

i.e., $R(t)=\sqrt{R_{0}^{2}-2 c t}$, valid until the extinction time $t=R_{0}^{2} / 2 c$, when the radius vanishes.

A heuristic arguments suggests that the variation of the perimeter be linked to the notion of curvature; hence, we expect to be able to obtain motion by mean curvature as a minimizing movement for the perimeter functional. We will see that in order to obtain geometric motions as minimizing movements we will have to modify the procedure described in the previous chapter.

Example 8.1.1 (pinning for the perimeter motion) Let $n=2$. We apply the minimizing-movement procedure to the perimeter functional (8.1) and the initial datum $A_{0}=$ $B_{R_{0}}(0)$ in $\mathbb{R}^{2}$. 
With fixed $\tau$, since

$$
\int_{\mathbb{R}^{2}}\left|\chi_{A}-\chi_{B}\right|^{2} d x=|A \triangle B|
$$

the minimization to determine $A_{1}$ is

$$
\min \left\{\mathcal{H}^{1}(\partial A)+\frac{1}{2 \tau}\left|A \triangle A_{0}\right|\right\} .
$$

We note that we can restrict our attention to sets $A$ contained in $A_{0}$, since otherwise taking $A \cap A_{0}$ as test sets in their place would decrease both terms in the minimization. Once this is observed, we also note that, given $A \subset A_{0}$, if $B_{R}(x) \subset A_{0}$ has the same measure as $A$ then it decreases the perimeter part of the energy (strictly, if $A$ itself is not a ball) while keeping the second term fixed. Hence, we can limit our analysis to balls $B_{R}(x) \subset A_{0}$, for which the energy depends only on $R$. The incremental problem is then given by

$$
\min \left\{2 \pi R+\frac{\pi}{2 \tau}\left(R_{0}^{2}-R^{2}\right): 0 \leq R \leq R_{0}\right\}
$$

whose minimizer is either $R=0$ (with value $\frac{\pi}{2 \tau} R_{0}^{2}$ ) or $R=R_{0}$ (with value $2 \pi R_{0}$ ) since in (8.4) we are minimizing a concave function of $R$. For $\tau$ small the minimizer is then $R_{0}$. This means that the motion is trivial: $A_{k}=A_{0}$ for all $k$, and hence also the resulting minimizing movement is trivial.

\subsection{A first (unsuccessful) generalization}

We may generalize the scheme of the minimizing movements by taking a more general distance term in the minimization; e.g., considering $x_{k}$ as a minimizer of

$$
\min \left\{F(x)+\frac{1}{\tau} \Phi\left(\left\|x-x_{k-1}\right\|\right)\right\}
$$

where $\Phi$ is a continuous increasing function with $\Phi(0)=0$. As an example, we can consider

$$
\Phi(z)=\frac{1}{p}|z|^{p} .
$$

Note that in this case we obtain the estimate

$$
\left\|x_{k}-x_{k-1}\right\|^{p} \leq p \tau\left(F\left(x_{k-1}\right)-F\left(x_{k}\right)\right)
$$

for the minimizer $x_{k}$. Using Hölder's inequality as in the case $p=2$, we end up with (for $j>h)$

$$
\begin{aligned}
\left\|x_{j}-x_{h}\right\| & \leq(j-h)^{(p-1) / p}\left(\sum_{k=h+1}^{j}\left\|x_{k}-x_{k-1}\right\|^{p}\right)^{1 / p} \\
& \leq\left(p F\left(x_{0}\right)\right)^{1 / p}\left(\tau^{1 /(p-1)}(j-h)\right)^{(p-1) / p} .
\end{aligned}
$$


In order to obtain a $\left(1-\frac{1}{p}\right)$ Hölder continuity for the interpolated function $u^{\tau}$ we have to define it as

$$
u^{\tau}(t)=u_{\left\lfloor t / \tau^{1 /(p-1)}\right\rfloor}
$$

Note that we may use the previous definition $u^{\tau}(t)=u_{\lfloor t / \tau\rfloor}$ for the interpolated function if we change the parameter $\tau$ in (8.5) and consider instead the problem

$$
\min \left\{F(x)+\frac{1}{\tau^{p-1}} \Phi\left(\left\|x-x_{k-1}\right\|\right)\right\}
$$

to define $x_{k}$.

Example 8.2.1 ((non-)geometric minimizing movements) We use the scheme above, with a slight variation in the exponents since we will be interested in the description of the motion in terms of the radius of a ball in $\mathbb{R}^{2}$ (which is the square root of the $L^{2}$ norm and not the norm itself). As in the previous example, we take the initial datum $A_{0}=B_{R_{0}}=B_{R_{0}}(0)$, and consider $A_{k}$ defined recursively as a minimizer of

$$
\min \left\{\mathcal{H}^{1}(\partial A)+\frac{1}{p \tau^{p-1}}\left|A \triangle A_{0}\right|^{p}\right\}
$$

with $p>1$. As above, at each step the minimizer is given by balls

$$
B_{R_{k}}\left(x_{k}\right) \subset B_{R_{k-1}}\left(x_{k-1}\right) .
$$

The value of $R_{k}$ is determined by solving

$$
\min \left\{2 \pi R+\frac{\pi^{p}}{p \tau^{p-1}}\left(R_{k-1}^{2}-R^{2}\right)^{p}: 0 \leq R \leq R_{k-1}\right\}
$$

which gives

$$
\frac{R_{k}-R_{k-1}}{\tau}=-\frac{1}{\pi R_{k}^{1 /(p-1)}\left(R_{k}+R_{k-1}\right)} .
$$

Note that in this case the minimum value is not taken at $R_{k}=R_{k-1}$ (this can be checked, e.g., by checking that the derivative of the function to be minimized in (8.9) is positive at $\left.R_{k-1}\right)$. By passing to the limit in (8.10) we deduce the equation

$$
R^{\prime}=-\frac{1}{2 \pi R^{p /(p-1)}}
$$

(valid until the extinction time).

Despite having obtained an equation for $R$ we notice that this approach is not satisfactory, since we have

- (non-geometric motion) in (8.8) we have infinitely many solutions; namely, all balls centered in $x_{k}$ with

$$
\left|x_{k-1}-x_{k}\right| \leq R_{k-1}-R_{k} .
$$


This implies that we may have moving centres $x(t)$ provided that $\left|x^{\prime}\right| \leq R^{\prime}$ and $x(0)=0$; in particular we may choose $x(t)=\left(R_{0}-R(t)\right) z$ for any $z \in B_{1}(0)$ which converges to $R_{0} z$; i.e., the point where the sets concentrate at the vanishing time may be any point in $\bar{B}_{R_{0}}$ at the extinction time. This implies that the motion is not a geometric one: sets do not move according to geometric quantities.

- (failure to obtain mean-curvature motion) even if we obtain an equation for $R$ we never obtain the mean curvature flow since $p /(p-1)>1$.

\subsection{A variational approach to curvature-driven motion}

In order to obtain motion by curvature Almgren, Taylor and Wang have introduced a variation of the implicit-time scheme described above, where the term $\left|A \triangle A_{k}\right|$ is substituted by an integral term which favours variations which are 'uniformly distant' to the boundary of $A_{k}$. The problem defining $A_{k}$ is then

$$
\min \left\{\mathcal{H}^{1}(\partial A)+\frac{1}{\tau} \int_{A \triangle A_{k-1}} \operatorname{dist}\left(x, \partial A_{k-1}\right) d x\right\} .
$$

We will not prove a general convergence result for an arbitrary initial datum $A_{0}$, but we will check the convergence to mean-curvature motion for $A=B_{R_{0}}$ in $\mathbb{R}^{2}$.

In this case we note that if $A_{k-1}$ is a ball centered in 0 then we have

- $A_{k}$ is contained in $A_{k-1}$. To check this note that, given a test set $A$, considering $A \cap A_{k-1}$ as a test set in its place decreases the energy in (8.12), strictly if $A \backslash A_{k-1} \neq \emptyset$;

- $A_{k}$ is convex and with baricenter in 0 . To check this, first, note that each connected component of $A_{k}$ is convex. Otherwise, considering the convex envelopes decreases the energy (strictly, if one of the connected components if not convex). Then note that if 0 is not the baricenter of a connected component of $A_{k}$ then a small translation towards 0 strictly decreases the energy (this follows by computing the derivative of the volume term along the translation). In particular, we only have one (convex) connected component;

From these properties we can conclude that $A_{k}$ is indeed a ball centered in 0 . Were it not so, there would be a line through 0 such that the boundary of $A_{k}$ does not intersect perpendicularly this line. By a reflection argument we then obtain a non-convex set $\widetilde{A}_{k}$ with total energy not greater than the one of $A_{k}$ (note that the line considered subdivides $A_{k}$ into two subsets with equal total energy). Its convexification would then strictly decrease the energy. This shows that each $A_{k}$ is of the form

$$
A_{k}=B_{R_{k}}=B_{R_{k}}(0) .
$$

We can now compute the equation satisfied by $R_{k}$, by minimizing (after passing to polar coordinates)

$$
\min \left\{2 \pi R+\frac{2 \pi}{\tau} \int_{R}^{R_{k-1}}\left(R_{k-1}-\rho\right) \rho d \rho\right\}
$$


which gives

$$
\frac{R_{k}-R_{k-1}}{\tau}=-\frac{1}{R_{k}}
$$

Passing to the limit gives the desired mean curvature equation (8.2).

\subsection{Homogenization of flat flows}

We now consider geometric functionals with many local minimizers (introduced in Example 3.5.1) which give a more refined example of homogenization. The functionals we consider are defined on (sufficiently regular) subsets of $\mathbb{R}^{2}$ by

$$
F_{\varepsilon}(A)=\int_{\partial A} a\left(\frac{x}{\varepsilon}\right) d \mathcal{H}^{1}
$$

where

$$
a\left(x_{1}, x_{2}\right)= \begin{cases}1 & \text { if } x_{1} \in \mathbb{Z} \text { or } x_{2} \in \mathbb{Z} \\ 2 & \text { otherwise. }\end{cases}
$$

The $\Gamma$-limit of the energies $F_{\varepsilon}$ is the crystalline perimeter energy

$$
F(A)=\int_{\partial A}\|\nu\|_{1} d \mathcal{H}^{1}
$$

with $\left\|\left(\nu_{1}, \nu_{2}\right)\right\|_{1}=\left|\nu_{1}\right|+\left|\nu_{2}\right|$. A minimizing movement for $F$ is called a flat flow. We will first briefly describe it, and then compare it with the minimizing movements for $F_{\varepsilon}$.

\subsubsection{Motion by crystalline curvature}

The incremental problems for the minimizing-movement scheme for $F$ in (8.16) are of the form

$$
\min \left\{F(A)+\frac{1}{\tau} \int_{A \triangle A_{k-1}} \operatorname{dist}_{\infty}\left(x, \partial A_{k-1}\right) d x\right\}
$$

where for technical reasons we consider the $\infty$-distance

$$
\operatorname{dist}_{\infty}(x, B)=\inf \left\{\|x-y\|_{\infty}: y \in B\right\} .
$$

However, in the simplified situation below this will not be relevant in our computations.

We only consider the case of an initial datum $A_{0}$ a rectangle, which plays the role played by a ball for motion by mean curvature. Note that, as in Section 8.3, we can prove that if $A_{k-1}$ is a rectangle, then we can limit the computation in (8.17) to

- $A$ contained in $A_{k-1}$ (otherwise $A \cap A_{k-1}$ strictly decreases the energy)

- $A$ with each connected component a rectangle (otherwise taking the least rectangle containing a given component would decrease the energy, strictly if $A$ is not a rectangle); 


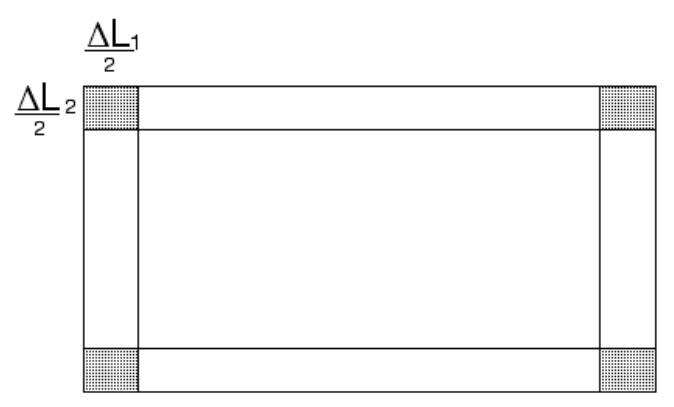

Figure 8.1: incremental crystalline minimization

- $A$ connected and with the same center as $A_{0}$ (since translating the center towards 0 decreases the energy).

Hence, we may suppose that

$$
A_{k}=\left[-\frac{L_{k, 1}}{2}, \frac{L_{k, 1}}{2}\right] \times\left[-\frac{L_{k, 2}}{2}, \frac{L_{k, 2}}{2}\right]
$$

for all $k$. In order to iteratively determine $L_{k}$ we have to minimize the energy

$$
\min \left\{2\left(L_{k, 1}+\Delta L_{1}\right)+2\left(L_{k, 2}+\Delta L_{2}\right)+\frac{1}{\tau} \int_{A \triangle A_{k-1}} \operatorname{dist}_{\infty}\left(x, \partial A_{k-1}\right) d x\right\} .
$$

In this computation it is easily seen that for $\tau$ small the integral term can be substituted by

$$
\frac{L_{k, 1}}{4}\left(\Delta L_{2}\right)^{2}+\frac{L_{k, 2}}{4}\left(\Delta L_{1}\right)^{2}
$$

This argument amounts to noticing that the contribution of the small rectangles at the corners highlighted in Figure 8.1 is negligible as $\tau \rightarrow 0$. The optimal increments (more precisely, decrements) $\Delta L_{j}$ are then determined by the conditions

$$
\left\{\begin{array}{l}
1+\frac{L_{k, 2}}{4 \tau} \Delta L_{1}=0 \\
1+\frac{L_{k, 1}}{4 \tau} \Delta L_{2}=0
\end{array}\right.
$$

Hence, we have the difference equations

$$
\frac{\Delta L_{1}}{\tau}=-\frac{4}{L_{k, 2}}, \quad \frac{\Delta L_{2}}{\tau}=-\frac{4}{L_{k, 1}}
$$


which finally gives the system of ODEs for the limit rectangles, with edges of length $L_{1}(t)$ and $L_{2}(t)$ respectively,

$$
\left\{\begin{array}{l}
L_{1}^{\prime}=-\frac{4}{L_{2}} \\
L_{2}^{\prime}=-\frac{4}{L_{1}} .
\end{array}\right.
$$

Geometrically, each edge of the rectangle moves inwards with velocity inversely proportional to its length; more precisely, equal to twice the inverse of its length (so that the other edge contracts with twice this velocity). Hence, the inverse of the length of an edge plays the role of the curvature in this context (crystalline curvature).

It is worth noticing that by (8.21) all rectangles are homothetic, since $\frac{d}{d t} \frac{L_{1}}{L_{2}}=0$, and with area satisfying

$$
\frac{d}{d t} L_{1} L_{2}=-8
$$

so that $L_{1}(t) L_{2}(t)=L_{0,1} L_{0,2}-8 t$, which gives the extinction time $t=L_{0,1} L_{0,2} / 8$. In the case of an initial datum a square of side length $L_{0}$, the sets are squares whose side length at time $t$ is given by $L(t)=\sqrt{L_{0}^{2}-8 t}$ in analogy with the evolution of balls by mean curvature flow.

\subsection{Homogenization of oscillating perimeters}

We consider the sequence $F_{\varepsilon}$ in (8.15). Note that for any (sufficiently regular) initial datum $A_{0}$ we have that $A_{\varepsilon}^{\prime} \subset A_{0} \subset A_{\varepsilon}^{\prime \prime}$, where $A_{\varepsilon}^{\prime}$ and $A_{\varepsilon}^{\prime \prime}$ are such that $F_{\varepsilon}\left(A_{\varepsilon}^{\prime}\right)=\mathcal{H}^{1}\left(\partial A_{\varepsilon}^{\prime}\right)$ and $F_{\varepsilon}\left(A_{\varepsilon}^{\prime \prime}\right)=\mathcal{H}^{1}\left(\partial A_{\varepsilon}^{\prime \prime}\right)$ and $\left|A_{\varepsilon}^{\prime \prime} \backslash A_{\varepsilon}^{\prime}\right|=O(\varepsilon)$. Such sets are local minimizers for $F_{\varepsilon}$ and hence the minimizing movement of $F_{\varepsilon}$ from either of them is trivial. As a consequence, if $A_{\varepsilon}(t)$ is a minimizing movement for $F_{\varepsilon}$ from $A_{0}$ we have

$$
A_{\varepsilon}^{\prime} \subset A_{\varepsilon}(t) \subset A_{\varepsilon}^{\prime \prime}
$$

This shows that for any set $A_{0}$ the only limit $\lim _{\varepsilon \rightarrow 0} A_{\varepsilon}(t)$ of minimizing movements for $F_{\varepsilon}$ from $A_{0}$ is the trivial motion $A(t)=A_{0}$.

We now compute the minimizing movements along the sequence $F_{\varepsilon}$ with initial datum a rectangle, and compare it with the flat flow described in the previous section.

For simplicity of computation we deal with a constrained case, when

- for every $\varepsilon$ the initial datum $A_{0}=A_{0}^{\varepsilon}$ is a rectangle centered in 0 such that $F_{\varepsilon}(A)=$ $\mathcal{H}^{1}(\partial A)$ (i.e., its edge lengths $L_{0, j}$ belong to $2 \varepsilon \mathbb{Z}$ ). In analogy with $x_{0}$ in the example in Section 7.4, if this does not hold then either it does after one iteration or we have a pinned state $A_{k}=A_{0}$ for all $k$;

- all competing $A$ are rectangles with $F_{\varepsilon}(A)=\mathcal{H}^{1}(\partial A)$ centered in 0 . The fact that all competing sets are rectangles follows as for the flat flow in the previous section. The fact that $F_{\varepsilon}\left(A_{k}\right) \leq F_{\varepsilon}\left(A_{k-1}\right)$ then implies that the minimal rectangles satisfy $F_{\varepsilon}\left(A_{k}\right)=$ 
$\mathcal{H}^{1}\left(\partial A_{k}\right)$. The only real assumption at this point is that they are centered in 0 . This hypothesis can be removed, upon a slightly more complex computation, which would only make the arguments less clear.

After this simplifications, the incremental problem is exactly as in (8.17) since for competing sets we have $F_{\varepsilon}(A)=F(A)$, the only difference being that now $L_{k, 1}, L_{k, 2} \in 2 \varepsilon \mathbb{Z}$. The problem in terms of $\triangle L_{j}$, using the same simplification for (8.18) as in the previous section, is then

$$
\min \left\{2\left(L_{k, 1}+\Delta L_{1}\right)+2\left(L_{k, 2}+\Delta L_{2}\right)+\frac{L_{k, 1}}{4 \tau}\left(\Delta L_{2}\right)^{2}+\frac{L_{k, 2}}{4 \tau}\left(\Delta L_{1}\right)^{2}: \Delta L_{j} \in 2 \varepsilon \mathbb{Z}\right\} .
$$

This is a minimization problem for a parabola as the ones in Section 7.4 that gives

$$
\Delta L_{1}=-\left\lfloor\frac{4 \tau}{\varepsilon L_{k, 2}}+\frac{1}{2}\right\rfloor \varepsilon \text { if } \frac{4 \tau}{\varepsilon L_{k, 2}}+\frac{1}{2} \notin \mathbb{Z}
$$

(the other cases giving two solutions), and an analogous equation for $\Delta L_{2}$. Passing to the limit we have the system of ODEs, governed by the parameter

$$
w=\lim _{\varepsilon \rightarrow 0} \frac{\tau}{\varepsilon}
$$

(which we may suppose up to subsequences), which reads as

$$
\left\{\begin{array}{l}
L_{1}^{\prime}=-\frac{1}{w}\left\lfloor\frac{4 w}{L_{2}}+\frac{1}{2}\right\rfloor \\
L_{2}^{\prime}=-\frac{1}{w}\left\lfloor\frac{4 w}{L_{1}}+\frac{1}{2}\right\rfloor .
\end{array}\right.
$$

Note that the right-hand side is a discontinuous function of $L_{j}$, so some care must be taken at times $t$ when $\frac{4 w}{L_{j}(t)}+\frac{1}{2} \in \mathbb{Z}$. However, apart some exceptional cases, this condition holds only for a countable number of $t$, and is therefore negligible.

We can compare the resulting minimizing movements with the crystalline curvature flow, related to $F$.

- (total pinning) if $\tau<<\varepsilon(w=0)$ then we have $A(t)=A_{0}$;

- (crystalline curvature flow) if $\varepsilon<<\tau$ then we have the minimizing movements described in the previous section;

- (partial pinning/asymmetric curvature flow) if $0<w<+\infty$ then we have

(i) (total pinning) if both $L_{0, j}>8 w$ then the motion is trivial $A(t)=A_{0}$;

(ii) (partial pinning) if $L_{0,1}>8 w, L_{0,2}<8 w$ and $\frac{4 w}{L_{0,2}}+\frac{1}{2} \notin \mathbb{Z}$ then the horizontal edges do not move, but they contract with constant velocity until $L_{1}(t)=8 w$;

(iii) (asymmetric curvature flow) if $L_{0,1} \leq 8 w$ and $L_{0,2}<8 w$ then we have a unique motion with $A(t) \subset \subset A(s)$ if $t>s$, up to a finite extinction time. Note however that the sets $A(s)$ are nor homothetic, except for the trivial case when $A_{0}$ is a square. 
Some cases are not considered above, namely those when we do not have uniqueness of minimizers in the incremental problem. This may lead to a multiplicity of minimizing movements, as remarked in Section 7.4.

It is worthwhile to highlight that we may rewrite the equations for $L_{j}^{\prime}$ as a variation of the crystalline curvature flow; e.g., for $L_{1}^{\prime}$ we can write it as

$$
L_{1}^{\prime}=-f\left(\frac{L_{2}}{w}\right) \frac{4}{L_{2}}, \quad \text { with } f(z)=\frac{z}{4}\left\lfloor\frac{4}{z}+\frac{1}{2}\right\rfloor .
$$

This suggests that the 'relevant' homogenized problem is the one obtained for $\frac{\tau}{\varepsilon}=1$, as all the others can be obtained from this one by a scaling argument.

We note that the scheme can be applied to the evolution of more general sets, but the analysis of the rectangular case already highlights the new features deriving from the microscopic geometry.

\subsection{Flat flow with oscillating forcing term}

We now consider another minimizing-movement scheme linked to the functional $F$ in (8.16). In this case the oscillations are given by a lower-order forcing term. We consider, in $\mathbb{R}^{2}$,

$$
G_{\varepsilon}(A)=\int_{\partial A}\|\nu\|_{1} d \mathcal{H}^{1}+\int_{A} g\left(\frac{x_{1}}{\varepsilon}\right) d x
$$

where $g$ is 1-periodic and even, given by

$$
g(s)= \begin{cases}\alpha & \text { if } \operatorname{dist}(x, \mathbb{Z})<\frac{1}{4} \\ \beta & \text { otherwise }\end{cases}
$$

with $\alpha, \beta \in \mathbb{R}$ and $\alpha<\beta$. Note that the additional term may be negative, so that this functional is not positive; however, the minimizing-movement scheme can be applied unchanged.

Since the additional term converges continuously in $L^{1}$ as $\varepsilon \rightarrow 0$, the $\Gamma$-limit is simply

$$
G(A)=\int_{\partial A}\|\nu\|_{1} d \mathcal{H}^{1}+\frac{\alpha+\beta}{2}|A| .
$$

\subsubsection{Flat flow with forcing term}

We now consider minimizing movements for $G$. As in Section 8.4.1 we only deal with a constrained problem, when both the initial datum and the competing sets are rectangles centered in 0. With the notation of Section 8.4.1 we are led to the minimum problem

$$
\min \left\{2\left(L_{k, 1}+\Delta L_{1}+L_{k, 2}+\Delta L_{2}\right)+\frac{L_{k, 1}}{4 \tau}\left(\Delta L_{2}\right)^{2}+\frac{L_{k, 2}}{4 \tau}\left(\Delta L_{1}\right)^{2}+\frac{\alpha+\beta}{2}\left(L_{k, 1}+\Delta L_{1}\right)\left(L_{k, 2}+\Delta L_{2}\right)\right\} .
$$


The minimizing pair $\left(\Delta L_{1}, \Delta L_{2}\right)$ satisfies

$$
\frac{\Delta L_{1}}{\tau}=-\left(\frac{4}{L_{k, 2}}+(\alpha+\beta)\left(1+\frac{\Delta L_{2}}{L_{k, 2}}\right)\right)
$$

and the analogous equation for $\frac{\Delta L_{2}}{\tau}$. Passing to the limit we have

$$
\left\{\begin{array}{l}
L_{1}^{\prime}=-\left(\frac{4}{L_{2}}+\alpha+\beta\right) \\
L_{2}^{\prime}=-\left(\frac{4}{L_{1}}+\alpha+\beta\right),
\end{array}\right.
$$

so that each edge moves with velocity $\frac{2}{L_{2}}+\frac{\alpha+\beta}{2}$, with the convention that it moves inwards if this number is positive, outwards if it is negative.

Note that if $\alpha+\beta \geq 0$ then $L_{1}$ and $L_{2}$ are always decreasing and we have finite-time extinction, while if $\alpha+\beta<0$ then we have an equilibrium for $L_{j}=\frac{4}{|\alpha+\beta|}$, and we have

expanding rectangles, with an asymptotic velocity of each side of $\frac{|\alpha+\beta|}{2}$ as the side length diverges.

\subsubsection{Homogenization of forcing terms}

In order to highlight new homogenization phenomena, we treat the case $\tau<<\varepsilon$ only. Again, we consider the constrained case when both the initial datum and the competing sets are rectangles centered in 0 and adopt the notation of Section 8.4.1.

Taking into account that $\tau<<\varepsilon$ the incremental minimum problem can be approximated by

$$
\begin{array}{r}
\min \left\{2\left(L_{k, 1}+\Delta L_{1}+L_{k, 2}+\Delta L_{2}\right)+\frac{L_{k, 1}}{4 \tau}\left(\Delta L_{2}\right)^{2}+\frac{L_{k, 2}}{4 \tau}\left(\Delta L_{1}\right)^{2}\right. \\
\left.+\frac{\alpha+\beta}{2} L_{k, 1} L_{k, 2}+\frac{\alpha+\beta}{2} L_{k, 1} \Delta L_{2}+g\left(\frac{L_{k, 1}}{2 \varepsilon}\right) L_{k, 2} \Delta L_{1}\right\}
\end{array}
$$

In considering the term $g\left(\frac{L_{k, 1}}{2 \varepsilon}\right)$ we assume implicitly that $\tau$ is so small that both $\frac{L_{k, 1}}{2 \varepsilon}$ and $\frac{L_{k, 1}+\Delta L_{1}}{2 \varepsilon}$ belong to the same interval where $g$ is constant. This can be assumed up to a number of $k$ that is negligible as $\tau \rightarrow 0$.

For the minimizing pair of (8.30) we have

$$
\left\{\begin{array}{l}
2+\frac{L_{k, 2}}{2 \tau} \Delta L_{1}+g\left(\frac{L_{k, 1}}{2 \varepsilon}\right) L_{k, 2}=0 \\
2+\frac{L_{k, 1}}{2 \tau} \Delta L_{2}+\frac{\alpha+\beta}{2} L_{k, 1}=0
\end{array}\right.
$$


that is,

$$
\left\{\begin{array}{l}
\frac{\Delta L_{1}}{\tau}=-\left(\frac{4}{L_{k, 2}}+2 g\left(\frac{L_{k, 1}}{2 \varepsilon}\right)\right) \\
\frac{\Delta L_{2}}{\tau}=-\left(\frac{4}{L_{k, 1}}+(\alpha+\beta)\right) .
\end{array}\right.
$$

This systems shows that the horizontal edges move with velocity $\frac{2}{L_{k, 1}}+\frac{\alpha+\beta}{2}$, while the velocity of the vertical edges depends on the location of the edge and is

$$
\frac{2}{L_{k, 2}}+g\left(\frac{L_{k, 1}}{2 \varepsilon}\right)
$$

We then deduce that the limit velocity for the horizontal edges of length $L_{1}$ is

$$
\frac{2}{L_{1}}+\frac{\alpha+\beta}{2}
$$

As for the vertical edges, we have:

- (mesoscopic pinning) if $L_{2}$ is such that

$$
\left(\frac{2}{L_{2}}+\alpha\right)\left(\frac{2}{L_{2}}+\beta\right)<0
$$

then the vertical edge is eventually pinned in the minimizing-movement scheme. This pinning is not due to the equality $L_{k+1,1}=L_{k, 1}$ in the incremental problem, but to the fact that the vertical edge move in different directions depending on the value of $g$;

- (homogenized velocity) if on the contrary the vertical edge length satisfies

$$
\left(\frac{2}{L_{2}}+\alpha\right)\left(\frac{2}{L_{2}}+\beta\right)>0
$$

then we have a limit effective velocity of the vertical edge given by the harmonic mean of the two velocities $\frac{2}{L_{2}}+\alpha$ and $\frac{2}{L_{2}}+\beta$; namely,

$$
\frac{\left(2+\alpha L_{2}\right)\left(2+\beta L_{2}\right)}{L_{2}\left(2+\frac{\alpha+\beta}{2} L_{2}\right)} .
$$

We finally examine some cases explicitly.

(i) Let $\alpha=-\beta$. Then we have

$$
\left\{\begin{array}{l}
L_{2}^{\prime}=-\frac{4}{L_{1}} \\
L_{1}^{\prime}=-2 \frac{\left(2-\beta L_{2}\right) \vee 0}{L_{2}}
\end{array}\right.
$$


i.e., the vertical edges are pinned if their length is larger than $2 / \beta$. In this case, the horizontal edges move inwards with constant velocity $\frac{2}{L_{0,1}}$. In this way the vertical edges shrink with rate $\frac{4}{L_{0,1}}$ until their length is $2 / \beta$. After this, the whole rectangle shrinks in all directions.

(ii) Let $\alpha<\beta<0$. Then for the vertical edges we have an interval of 'mesoscopic pinning' corresponding to

$$
\frac{2}{|\beta|} \leq L_{2} \leq \frac{2}{|\alpha|}
$$

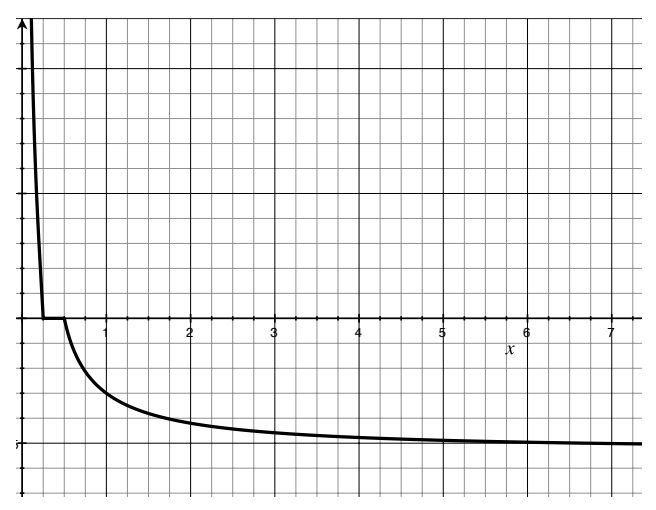

Figure 8.2: velocity with an interval of mesoscopic pinning

The velocity of the vertical edges in dependence of their length is then given by

$$
v= \begin{cases}0 & \text { if }(8.35) \text { holds } \\ \frac{\left(2+\alpha L_{2}\right)\left(2+\beta L_{2}\right)}{L_{2}\left(2+\frac{\alpha+\beta}{2} L_{2}\right)} & \text { otherwise }\end{cases}
$$

and is pictured in Figure 8.2. Instead, the velocity of the horizontal edges is given by (8.33), so that they move inwards if

$$
L_{1}<\frac{4}{|\alpha+\beta|}
$$

and outwards if $L_{1}>\frac{4}{|\alpha+\beta|}$.

In this case we can consider as initial datum a square of side length $L_{0}$.

If $L_{0} \leq \frac{2}{|\beta|}$ then all edges move inwards until a finite extinction time;

if $\frac{2}{|\beta|}<L_{0}<\frac{4}{|\alpha+\beta|}$ then first only the horizontal edges move inwards until the vertical edge reaches the length $\frac{2}{|\beta|}$, after which all edges move inwards; 
if $\frac{4}{|\alpha+\beta|}<L_{0}<\frac{2}{|\alpha|}$ then first only the horizontal edges move outwards until the vertical edge reaches the length $\frac{2}{|\alpha|}$, after which all edges move outwards;

if $L_{0} \geq \frac{2}{|\alpha|}$ then all edges move outwards, and the motion is defined for all times. The asymptotic velocity of the vertical edges as the length of the edges diverges is

$$
\left|\frac{2 \alpha \beta}{\alpha+\beta}\right|
$$

lower than $\left|\frac{\alpha+\beta}{2}\right|$ (the asymptotic velocity for the horizontal edges).

The critical case can be shown to be $\varepsilon \sim \tau$, so that for $\varepsilon<<\tau$ we have the flat flow with averaged forcing term described in Section 8.6.1. The actual description in the case $\varepsilon \sim \tau$ would involve a homogenization argument for the computation of the averaged velocity of vertical sides.

\subsection{References to Chapter 8}

The variational approach for motion by mean curvature is due to Almgren, Taylor, and Wang [2]. The variational approach for crystalline curvature flow is contained in a paper by Almgren and Taylor [1].

The homogenization of the flat flow essentially follows the discrete analog contained in the paper by Braides, Gelli, and Novaga [14]. In that paper more effects of the microscopic geometry are described for more general initial sets. The homogenization with forcing term is part of ongoing work with M. Novaga.

Geometric motions with a non-trivial homogenized velocity are described in the paper by Braides and Scilla [17]. 


\section{Chapter 9}

\section{Different time scales}

In this chapter we treat some variations on the minimizing-movement scheme motivated by some time-scaling argument.

\subsection{Long-time behaviour}

We will consider a new parameter $\lambda>0$ and follow the iterative minimizing scheme from an initial datum $x_{0}$ by considering $x_{k}$ defined recursively as a minimizer of

$$
\min \left\{\frac{1}{\lambda} F_{\varepsilon}(x)+\frac{1}{2 \tau}\left\|x-x_{k-1}\right\|^{2}\right\},
$$

and setting $u^{\tau}(t)=u^{\tau, \lambda}(t)=x_{\lfloor t / \tau\rfloor}$. Equivalently, we may view this as applying the minimizing-movement scheme to

$$
\min \left\{F_{\varepsilon}(x)+\frac{\lambda}{2 \tau}\left\|x-x_{k-1}\right\|^{2}\right\} .
$$

Note that we may compare this scheme with the usual one where $x_{i}$ are defined as minimizers of the minimizing-movement scheme with time scale $\eta=\tau / \lambda$ giving $u^{\eta}$ as a discretization with lattice step $\eta$. Then we have

$$
u^{\tau}(t)=x_{\lfloor t / \tau\rfloor}=x_{\lfloor t / \lambda \eta\rfloor}=u^{\eta}\left(\frac{t}{\lambda}\right) .
$$

Hence, the introduction of $\lambda$ corresponds to a scaling of time.

Remark 9.1.1 Note that the process described above may be meaningful also if $F_{\varepsilon}=F$ is independent of $\varepsilon$. In this case, as $\tau \rightarrow 0$ we obtain the minimizing movement along $F_{\lambda}=\frac{1}{\lambda} F$ with $\lambda$ in place of $\varepsilon$ in the notation used hitherto (of course, being a matter of notation, up to a change of parameters; i.e., considering $1 / \lambda$ in place of $\lambda$, we can always suppose that $\lambda \rightarrow 0$ ). 
Conversely, if $F_{\varepsilon} \Gamma$-converges to $F$ and $\lambda=\lambda_{\varepsilon}$ is a scale along which there exists a non-trivial $\Gamma$-development; i.e., the limit

$$
F^{(1)}(x)=\Gamma-\lim _{\varepsilon \rightarrow 0} \frac{F_{\varepsilon}(x)-\min F}{\lambda_{\varepsilon}}
$$

then from (9.1) we argue that such $\lambda_{\varepsilon}$ is a meaningful scale (which may be related to minimizing movements for $\left.F^{(1)}\right)$. More in general, such a scale is that suggested by the existence of a non-trivial

$$
\Gamma-\lim _{\varepsilon \rightarrow 0} \frac{F_{\varepsilon}(x)-m_{\varepsilon}}{\lambda_{\varepsilon}}
$$

for some choice of $m_{\varepsilon}$.

Finally, if $F_{\varepsilon}=F$, taking $\lambda=0$ in (9.2) we obtain a global minimum problem for $F$, while the trajectories $u^{\tau}(t / \lambda)$ may remain uniformly distant from a global minimizer for $\tau$ small (e.g., if the initial datum $x_{0}$ is a local minimizer for $F$ ). Similarly, for time-depending minimizing movements time-scaling in general does not lead to a quasistatic motion (as in Example 6.2.2)

We now first give some simple examples which motivate the study of time-scaled problems, also when the unscaled problems already give a non trivial minimizing movement.

Example 9.1.2 Consider in $\mathbb{R}^{2}$ the energy

$$
F_{\varepsilon}(x, y)=\frac{1}{2}\left(x^{2}+\varepsilon y^{2}\right) .
$$

The corresponding gradient flow is then

$$
\left\{\begin{array}{l}
x^{\prime}=-x \\
y^{\prime}=-\varepsilon y
\end{array}\right.
$$

with solutions of the form

$$
\left(x_{\varepsilon}(t), y_{\varepsilon}(t)\right)=\left(x_{0} e^{-t}, y_{0} e^{-\varepsilon t}\right) .
$$

These solutions converge to $(x(t), y(t))=\left(x_{0} e^{-t}, y_{0}\right)$, solving

$$
\left\{\begin{array}{l}
x^{\prime}=-x \\
y^{\prime}=0
\end{array}\right.
$$

which is the gradient flow of the limit $F(x, y)=\frac{1}{2} x^{2}$. Note that

$$
\lim _{t \rightarrow+\infty}\left(x_{\varepsilon}(t), y_{\varepsilon}(t)\right)=(0,0) \neq\left(0, y_{0}\right)=\lim _{t \rightarrow+\infty}(x(t), y(t)) .
$$




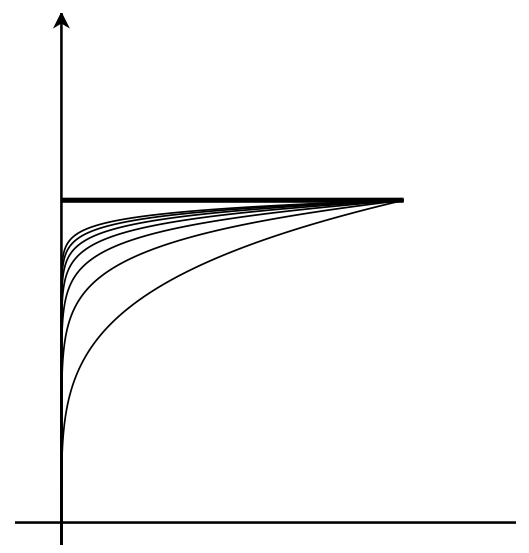

Figure 9.1: trajectories of the solutions, and their pointwise limit

The trajectories of the solutions $\left(x_{\varepsilon}, y_{\varepsilon}\right)$ lie on the curves

$$
\frac{y}{y_{0}}=\left(\frac{x}{x_{0}}\right)^{\varepsilon}
$$

and are pictured in Fig. 9.1.

The solutions can be seen as superposition of $(x(t), y(t))$ and $\varepsilon\left(x_{\infty}(t), y_{\infty}(t)\right)$, where

$$
\left(x_{\infty}(t), y_{\infty}(t)\right):=\left(0, e^{-t}\right)
$$

is the solution of

$$
\left\{\begin{array}{l}
x^{\prime}=0 \\
y^{\prime}=-y \\
(x(0), y(0))=\left(0, y_{0}\right) .
\end{array}\right.
$$

The solution $\left(x_{\infty}, y_{\infty}\right)$ can be obtained by scaling $\left(x_{\varepsilon}, y_{\varepsilon}\right)$; namely,

$$
\left(x_{\infty}(t), y_{\infty}(t)\right)=\lim _{\varepsilon \rightarrow 0}\left(x_{\varepsilon}(t / \varepsilon), y_{\varepsilon}(t / \varepsilon)\right)
$$

In this case the scaled time-scale is $\lambda=\varepsilon$. Note that the limit of the scaled solutions does not satisfy the original initial condition, but its 'projection' on the set of (local) minimizers of the limit energy $F$ (or, in other words, the domain of the limit of the energies $\frac{1}{\varepsilon} F_{\varepsilon}$ ).

Example 9.1.3 A similar example can be constructed in one dimension, taking, e.g.,

$$
F_{\varepsilon}(x)=\frac{\varepsilon}{2} x^{2}+\frac{1}{2}((|x|-1) \vee 0)^{2} .
$$

If $x_{0}<-1$ then the corresponding solutions $x_{\varepsilon}$ satisfy: 
- the limit $x(t)=\lim _{\varepsilon \rightarrow 0} x_{\varepsilon}(t)$ solves

$$
\left\{\begin{array}{l}
x^{\prime}=-x+1 \\
x(0)=x_{0}
\end{array}\right.
$$

which corresponds to the gradient flow of the energy

$$
F(x)=\frac{1}{2}((|x|-1) \vee 0)^{2} .
$$

- the scaled limit $x_{\infty}(t)=\lim _{\varepsilon \rightarrow 0} x_{\varepsilon}(t / \varepsilon)$ solves

$$
\left\{\begin{array}{l}
x^{\prime}=-x \\
x(0)=-1
\end{array}\right.
$$

which corresponds to the gradient flow of the energy

$$
F_{\infty}(x)=\lim _{\varepsilon \rightarrow 0} \frac{1}{\varepsilon} F_{\varepsilon}(x) .
$$

In this case the initial datum is the projection of $x_{0}$ on the domain of $F_{\infty}$.

Remark 9.1.4 In the previous examples we faced the problem of defining a minimizing movement for a sequence of functionals $F_{\varepsilon}(\Gamma$-)converging to a limit $F$ when the initial data $x_{0}^{\varepsilon}$ converge to a point $x_{0} \notin \operatorname{dom} F$. Note that in this case the approximating trajectories $u^{\varepsilon}$ are always defined if one can define $x_{1}^{\varepsilon}$; i.e., a solution of

$$
\min \left\{F_{\varepsilon}(x)+\frac{1}{2 \tau}\left\|x-x_{0}\right\|^{2}\right\}
$$

or equivalently of

$$
\min \left\{2 \tau F_{\varepsilon}(x)+\left\|x-x_{0}\right\|^{2}: x \in \operatorname{dom} F\right\},
$$

after which $x_{1}^{\tau} \in \operatorname{dom} F$ and we apply the theory already studied. Note that if $\operatorname{dom} F$ is a closed set in $X$ then $x_{1}^{\tau}$ converge to the projection $x_{1}$ of $x_{0}$ on dom $F$, so it may be meaningful to directly study the minimizing movements from that point. Note however that, as always, the choice of initial data $x_{1}^{\tau} \rightarrow x_{1}$ may provide a choice among the minimizing movements from $x_{1}$.

We now give more examples with families of energies $F_{\varepsilon} \Gamma$-converging to a limit $F$. Since we are mainly interested in highlighting the existence of a time scale $\lambda=\lambda_{\varepsilon}$ at which the scaled motion is not trivial, we will make some simplifying assumptions, one of which is that the initial datum be a local minimizer for $F$, so that the (unscaled) minimizing movement for the limit from that point is trivial. 
Example 9.1.5 We take as $F$ the 1D Mumford-Shah functional on $(0,1)$ defined by

$$
F(u)=\int_{0}^{1}\left|u^{\prime}\right|^{2} d t+\#(S(u))
$$

with domain the set of piecewise- $H^{1}$ functions. We take

$$
F_{\varepsilon}(u)=\int_{0}^{1}\left|u^{\prime}\right|^{2} d t+\sum_{S(u)} g\left(\frac{\left|u^{+}-u^{-}\right|}{\varepsilon}\right),
$$

where $g$ is a positive concave function with

$$
\lim _{z \rightarrow+\infty} g(z)=1 .
$$

We also consider the boundary conditions

$$
u(0-)=0, \quad u(1+)=1 .
$$

We suppose that

- $u_{0}$ is a local minimizer for $F$; i.e., it is piecewise constant;

- \# $\left(S\left(u_{0}\right)\right)=\left\{x_{0}, x_{1}\right\}$ (the simplest non-trivial local minimizer) with $0 \leq x_{0}<x_{1} \leq 1$;

- competing functions are also piecewise constant.

With these conditions, all minimizers $u_{k}$ obtained by iterative minimization satisfy:

- $S\left(u_{k}\right) \subset\left\{x_{0}, x_{1}\right\}$.

We may use the constant value $z_{k}$ of $u_{k}$ on $\left(x_{0}, x_{1}\right)$ as a one-dimensional parameter. The minimum problem defining $z_{k}$ is then (supposing that $z_{0}>0$ so that all $z_{k}>0$ )

$$
\min \left\{\frac{1}{\lambda}\left(g\left(\frac{z}{\varepsilon}\right)+g\left(\frac{1-z}{\varepsilon}\right)\right)+\frac{1}{2 \tau}\left(x_{1}-x_{0}\right)\left(z-z_{k-1}\right)^{2}\right\}
$$

which gives

$$
\left(x_{1}-x_{0}\right) \frac{z_{k}-z_{k-1}}{\tau}=-\frac{1}{\varepsilon \lambda}\left(g^{\prime}\left(\frac{z_{k}}{\varepsilon}\right)-g^{\prime}\left(\frac{1-z_{k}}{\varepsilon}\right)\right) .
$$

As an example, we may take

$$
g(z)=\frac{z}{1+z}
$$

so that the equation for $z_{k}$ becomes

$$
\left.\left(x_{1}-x_{0}\right) \frac{z_{k}-z_{k-1}}{\tau}=-\frac{\varepsilon}{\lambda}\left(\frac{1}{\varepsilon^{2}+z_{k}^{2}}-\frac{1}{\varepsilon^{2}+\left(z_{k}-1\right)^{2}}\right)\right) .
$$

This suggests the scale

$$
\lambda=\varepsilon,
$$


and with this choice gives the limit equation for $z(t)$

$$
z^{\prime}=-\frac{1-2 z}{\left(x_{1}-x_{0}\right) z^{2}(z-1)^{2}}
$$

In this time scale, unless we are in the equilibrium $z=\frac{1}{2}$ the middle value moves towards the closest value between 0 and 1 .

As a side remark, note that a simple qualitative study of this equation shows that if the initial datum is not $1 / 2$ then $z=0$ or $z=1$ after a finite time, after which the motion is trivial. Note that the limit state is a local minimum with only one jump.

Example 9.1.6 We consider the same functionals $F$ and $F_{\varepsilon}$ as in Example 9.1.5 with an initial datum with three jumps satisfying the same Dirichlet boundary conditions $u\left(0^{-}\right)=0, u\left(1^{+}\right)=1$ and the same assumptions as before.

With the notation used above, the minimum problem is

$$
\begin{aligned}
\min \left\{\frac{1}{\lambda}(\right. & \left.g\left(\frac{z_{0}-0}{\varepsilon}\right)+g\left(\frac{z_{1}-z_{0}}{\varepsilon}\right)+g\left(\frac{1-z_{1}}{\varepsilon}\right)\right) \\
& \left.+\frac{1}{2 \tau}\left(\left(x_{1}-x_{0}\right)\left|z_{0}-z_{0}^{k-1}\right|^{2}+\left(x_{2}-x_{1}\right)\left|z_{1}-z_{1}^{k-1}\right|^{2}\right)\right\} .
\end{aligned}
$$

Differently from the previous case, now we have to compute a gradient as a function of $z_{0}$ and $z_{1}$, the constant values of $u$ respectively on $\left(x_{0}, x_{1}\right)$ and $\left(x_{1}, x_{2}\right)$. Hence, the Euler equations for (9.3) give the following system for $z_{0}^{k}$ and $z_{1}^{k}$ :

$$
\begin{gathered}
\left(x_{1}-x_{0}\right) \frac{z_{0}^{k}-z_{0}^{k-1}}{\tau}=-\frac{1}{\lambda \varepsilon}\left(g^{\prime}\left(\frac{z_{0}^{k}}{\varepsilon}\right)-g^{\prime}\left(\frac{z_{1}^{k}-z_{0}^{k}}{\varepsilon}\right)\right), \\
\left(x_{2}-x_{1}\right) \frac{z_{1}^{k}-z_{1}^{k-1}}{\tau}=-\frac{1}{\lambda \varepsilon}\left(g^{\prime}\left(\frac{z_{1}^{k}-z_{0}^{k}}{\varepsilon}\right)-g^{\prime}\left(\frac{1-z_{1}^{k}}{\varepsilon}\right)\right) .
\end{gathered}
$$

For the sake of illustration, we may take the same $g$ as in the previous example, so that equations (9.4) and (9.5) become

$$
\begin{aligned}
& \left(x_{1}-x_{0}\right) \frac{z_{0}^{k}-z_{0}^{k-1}}{\tau}=-\frac{\varepsilon}{\lambda}\left(\frac{1}{\left(\varepsilon+z_{0}^{k}\right)^{2}}-\frac{1}{\left(\varepsilon+z_{1}^{k}-z_{0}^{k}\right)^{2}}\right), \\
& \left(x_{2}-x_{1}\right) \frac{z_{1}^{k}-z_{1}^{k-1}}{\tau}=-\frac{\varepsilon}{\lambda}\left(\frac{1}{\left(\varepsilon+z_{1}^{k}-z_{0}^{k}\right)^{2}}-\frac{1}{\left(\varepsilon+1-z_{1}^{k}\right)^{2}}\right) .
\end{aligned}
$$

This suggests the scale

$$
\lambda=\varepsilon
$$


and with this choice the limit equations for $z_{0}(t)$ and $z_{1}(t)$ are

$$
\begin{aligned}
z_{0}^{\prime} & =-\frac{z_{1}\left(z_{1}-2 z_{0}\right)}{\left(x_{1}-x_{0}\right) z_{0}^{2}\left(z_{1}-z_{0}\right)^{2}} \\
z_{1}^{\prime} & =-\frac{1-z_{0}^{2}-2 z_{1}\left(1-z_{0}\right)}{\left(x_{2}-x_{1}\right)\left(z_{1}-z_{0}\right)^{2}\left(1-z_{1}\right)^{2}} .
\end{aligned}
$$

In this time scale, it is easy to see that the gradient is zero when $\left(z_{0}, z_{1}\right)=\left(\frac{z_{1}}{2}, \frac{1+z_{0}}{2}\right)$, so we can have the following different behaviors:

- Equilibrium point. For the initial datum $\left(\bar{z}_{0}, \bar{z}_{1}\right)=\left(\frac{1}{3}, \frac{2}{3}\right)$ the motion is trivial;

- If $z_{0}$ is larger than the equilibrium point, then $z_{0}^{\prime}>0$ and the constant value $z_{0}$ will increase towards $z_{1}$, otherwise it will decrease towards zero. The same holds for $z_{1}$ between $z_{0}$ and 1 .

It must be noted that if the initial datum is not an equilibrium point then after a finite time one of the jump sizes vanishes, after which we are back to the previous example. In Figures $9.2-9.5$ we picture four stages of the evolution computed numerically.

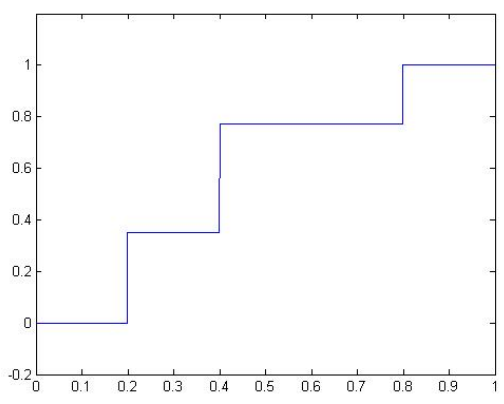

Figure 9.2: Initial conditions

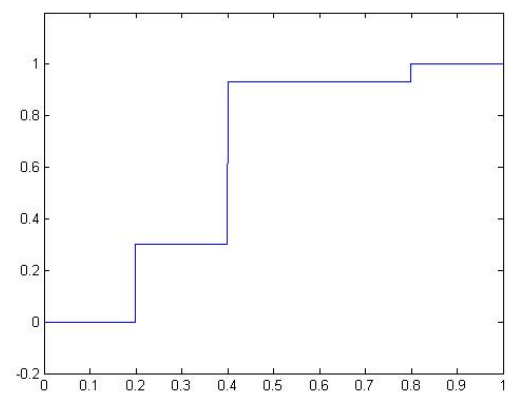

Figure 9.3: Iteration n. 30

A further simplified example is obtained by taking symmetric initial data $x_{2}-x_{1}=$ $x_{1}-x_{0}=: L$ and $z_{0}(0)=\frac{1}{2}-w_{0}$ and $z_{1}(0)=\frac{1}{2}+w_{0}$ with $0<w_{0}<1 / 2$, for which the motion is described by a single parameter $w(t)$ satisfying

$$
w^{\prime}=\frac{3\left(\frac{1}{2}+w\right)\left(w-\frac{1}{6}\right)}{4 L\left(\frac{1}{2}-w\right) w^{2}},
$$

in which case the equilibrium point corresponds to $w_{0}=1 / 6$, and otherwise after a finite either we have $w=0$ (which gives $z_{0}=z_{1}=1 / 2$; i.e., the equilibrium point with two jumps) or $w=\frac{1}{2}$ (which gives $z_{0}=0$ and $z_{1}=1$; i.e., a final state with only one jump point 


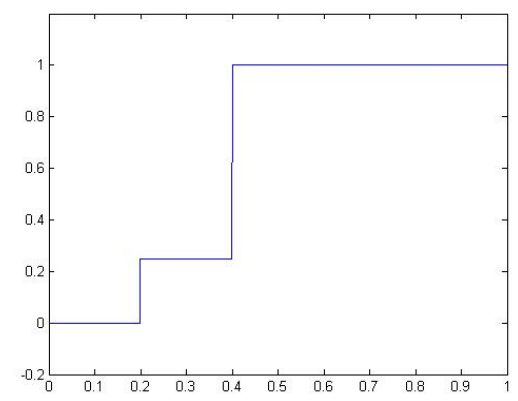

Figure 9.4: Iteration n. 60

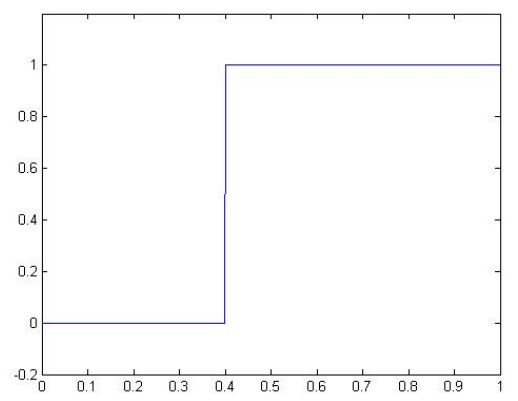

Figure 9.5: Iteration n. 100

Example 9.1.7 We consider another approximation of the Mumford-Shah functional: the (scaled) Perona-Malik functional. In the notation for discrete functionals (see Section 3.4), we may define

$$
F_{\varepsilon}(u)=\sum_{i=1}^{N} \frac{1}{|\log \varepsilon|} \log \left(1+\varepsilon|\log \varepsilon|\left|\frac{u_{i}-u_{i-1}}{\varepsilon}\right|^{2}\right) .
$$

Note that also the pointwise limit on piecewise- $H^{1}$ functions gives the Mumford-Shah functional since

$$
\lim _{\varepsilon \rightarrow 0} \frac{1}{\varepsilon|\log \varepsilon|} \log \left(1+\varepsilon|\log \varepsilon| z^{2}\right)=z^{2}
$$

and

$$
\lim _{\varepsilon \rightarrow 0} \frac{1}{|\log \varepsilon|} \log \left(1+|\log \varepsilon| \frac{w^{2}}{\varepsilon}\right)=1
$$

for all $w \neq 0$.

As in the Example 9.1.5, we consider the case when competing functions are nonnegative piecewise constants with $S(u) \subset S\left(u_{0}\right)=\left\{x_{0}, x_{1}\right\}$ and with the boundary conditions $u(0-)=0, u(1+)=1$. The computation is then reduced to a one-dimensional problem with unknown the constant value $z_{k}$ defined by the minimization

$$
\min \left\{\frac{1}{\lambda|\log \varepsilon|}\left(\log \left(1+|\log \varepsilon| \frac{z^{2}}{\varepsilon}\right)+\log \left(1+|\log \varepsilon| \frac{(z-1)^{2}}{\varepsilon}\right)\right)+\frac{1}{2 \tau}\left(x_{1}-x_{0}\right)\left(z-z_{k-1}\right)^{2}\right\},
$$

which gives the equation

$$
\left(x_{1}-x_{0}\right) \frac{z_{k}-z_{k-1}}{\tau}=-\frac{2}{\lambda}\left(\frac{z}{\varepsilon+|\log \varepsilon| z^{2}}+\frac{z-1}{\varepsilon+|\log \varepsilon|(z-1)^{2}}\right) .
$$

This suggests the time scale

$$
\lambda=\frac{1}{|\log \varepsilon|},
$$


and gives the equation for $z(t)$

$$
z^{\prime}=-\frac{2}{\left(x_{1}-x_{0}\right)} \cdot \frac{1-2 z}{z(1-z)}
$$

which provides a qualitative behaviour of $z$ similar to the previous example.

Example 9.1.8 We now consider the sharp-interface model with

$$
F(u)=\#(S(u) \cap[0,1))
$$

defined on all piecewise-constant 1-periodic functions with values in \pm 1 . For $F$ all functions are local minimizers.

We take

$$
F_{\varepsilon}(u)=\#(S(u) \cap[0,1))-\sum_{x_{i} \in[0,1) \cap S(u)} e^{-\frac{x_{i+1}-x_{i}}{\varepsilon}},
$$

where $\left\{x_{i}\right\}=S(u)$ is a numbering of $S(u)$ with $x_{i}<x_{i+1}$.

We take as initial datum $u_{0}$ with $\#\left(S\left(u_{0}\right)\right)=2$; hence, $S\left(u_{0}\right)=\left\{x_{0}, y_{0}\right\}$, and, after identifying $u_{0}$ with $A_{0}=\left[x_{0}, y_{0}\right]$, apply the Almgren-Taylor-Wang variant of the iterative minimization process, where the distance term $\frac{1}{2 \tau}\left\|u-u_{k-1}\right\|^{2}$ is substituted by

$$
\frac{1}{\tau} \int_{A \triangle A_{k-1}} \operatorname{dist}\left(x, \partial A_{k-1}\right) d x .
$$

The computation of $A_{1}=\left[x_{1}, y_{1}\right]$ is obtained by the minimization problem

$$
\min \left\{-\frac{1}{\lambda}\left(e^{-\frac{(y-x)}{\varepsilon}}+e^{-\frac{(1+x-y)}{\varepsilon}}\right)+\frac{1}{2 \tau}\left(\left(x-x_{0}\right)^{2}+\left(y-y_{0}\right)^{2}\right)\right\},
$$

which gives

$$
\begin{aligned}
& \frac{x_{1}-x_{0}}{\tau}=\frac{1}{\varepsilon \lambda}\left(e^{-\frac{\left(y_{1}-x_{1}\right)}{\varepsilon}}-e^{-\frac{\left(1+x_{1}-y_{1}\right)}{\varepsilon}}\right) \\
& \frac{y_{1}-y_{0}}{\tau}=-\frac{1}{\varepsilon \lambda}\left(e^{-\frac{\left(y_{1}-x_{1}\right)}{\varepsilon}}-e^{-\frac{\left(1+x_{1}-y_{1}\right)}{\varepsilon}}\right) .
\end{aligned}
$$

Let $y_{0}-x_{0}<1 / 2$; we argue that the scaled time scale is

$$
\lambda=\frac{1}{\varepsilon} e^{-\frac{y_{0}-x_{0}}{\varepsilon}}
$$

for which we have

$$
\begin{aligned}
& \frac{x_{1}-x_{0}}{\tau}=\left(e^{-\frac{\left(y_{1}-y_{0}-x_{1}+x_{0}\right)}{\varepsilon}}-e^{-\frac{\left(1+x_{1}-x_{0}-y_{1}+y_{0}\right)}{\varepsilon}}\right) \\
& \frac{y_{1}-y_{0}}{\tau}=-\left(e^{-\frac{\left(y_{1}-y_{0}-x_{1}+x_{0}\right)}{\varepsilon}}-e^{-\frac{\left(1+x_{1}-x_{0}-y_{1}+y_{0}\right)}{\varepsilon}}\right) .
\end{aligned}
$$


In terms of $L_{k}=y_{k}-x_{k}$ this can be written as

$$
\frac{L_{1}-L_{0}}{\tau}=-2\left(e^{-\frac{\left(L_{1}-L_{0}\right)}{\varepsilon}}-e^{-\frac{\left(1+L_{0}-L_{1}\right)}{\varepsilon}}\right) .
$$

Under the assumption $\tau<<\varepsilon$ we have in the limit

$$
L^{\prime}=-2\left(e^{o(1)}-e^{-\frac{1}{\varepsilon}+o(1)}\right)=-2,
$$

which shows that the two closer interfaces move towards each other shortening linearly their distance.

\subsection{Reversed time}

In a finite-dimensional setting a condition to be able to define a minimizing movement for $F$ is that

$$
u \mapsto F(u)+\frac{1}{2 \tau}|u-\bar{u}|^{2}
$$

be lower semicontinuous and coercive for all $\bar{u}$ and for $\tau$ sufficiently small. This is not in contrast with requiring that also

$$
u \mapsto-F(u)+\frac{1}{2 \tau}|u-\bar{u}|^{2}
$$

satisfy the same conditions; for example if $F$ is continuous and of quadratic growth. Note that this can be seen as a further extension of the time-scaling argument in the previous sections with $\lambda=-1$. If the iterative scheme gives a solution for the gradient flow, a minimizing movement $u$ for the second scheme produces a solution $v(t)=u(-t)$ to the backward problem

$$
\left\{\begin{array}{l}
v^{\prime}(t)=-F(v(t)) \quad \text { for } t \leq 0 \\
v(0)=u_{0}
\end{array}\right.
$$

In an infinite-dimensional setting the two requirements of being able to define both the minimizing movement (9.11) and (9.12) greatly limits the choice of $F$, and rules out all interesting cases. A possible approach to the definition of a backward minimizing movement is then to introduce a (finite-dimensional) approximation $F_{\varepsilon}$ to $F$, for which we can define a minimizing motion along $-F_{\varepsilon}$.

We now give an example in the context of crystalline motion, where we consider a negative scaling of time.

Example 9.2.1 We consider in $\mathbb{R}^{2}$

$$
F(A)=\int_{\partial A}\|\nu\|_{1} d \mathcal{H}^{1},
$$


and $F_{\varepsilon}$ the restriction of $F$ to the sets of the form

$$
\bigcup\left\{\varepsilon i+\left(-\frac{\varepsilon}{2}, \frac{\varepsilon}{2}\right)^{2}: i \in B\right\}
$$

where $B$ is a subset of $\mathbb{Z}^{2}$. Hence, we may identify these union of $\varepsilon$-cubes with the corresponding $B$. Even though this is not a finite-dimensional space, we will be able to apply the Almgren-Taylor-Wang scheme.

We choose (with the identifications with subsets of $\mathbb{Z}^{2}$ ) as initial datum

$$
A_{0}^{\varepsilon}=\{(0,0)\}=\left(-\frac{\varepsilon}{2}, \frac{\varepsilon}{2}\right)^{2}
$$

and solve iteratively

$$
\min \left\{-\frac{1}{\lambda} F_{\varepsilon}(A)+\frac{1}{\tau} \int_{A \backslash A_{k-1}^{\varepsilon}} \operatorname{dist}_{\infty}\left(x, \partial A_{k-1}^{\varepsilon}\right) d x\right\} .
$$

with $\lambda=\lambda_{\varepsilon}>0$ to be determined. In the interpretation as a reversed-time scheme, this means that we are solving a problem imposing the extinction at time 0 .

Note that taking $F$ in place of $F_{\varepsilon}$ would immediately give the value $-\infty$ in the minimum problem above; e.g., by considering sets of the form (in polar coordinates)

$$
A_{j}=\{(\rho, \theta): \rho \leq 3 \varepsilon+\varepsilon \sin (j \theta)\}
$$

which contain $A_{0}^{\varepsilon}$, are contained in $B_{4 \varepsilon}(0)$ and have a perimeter larger than $4 j \varepsilon$.

Under the assumption that $\varepsilon<<\tau$ all minimizing sets are the checkerboard structure corresponding to indices $i \in \mathbb{Z}^{2}$ with $i_{1}+i_{2}$ even contained in a square $Q_{k}$ centered in 0 (see Fig. 9.6). We may take the sides $L_{k}$ of those squares as unknown. The incremental
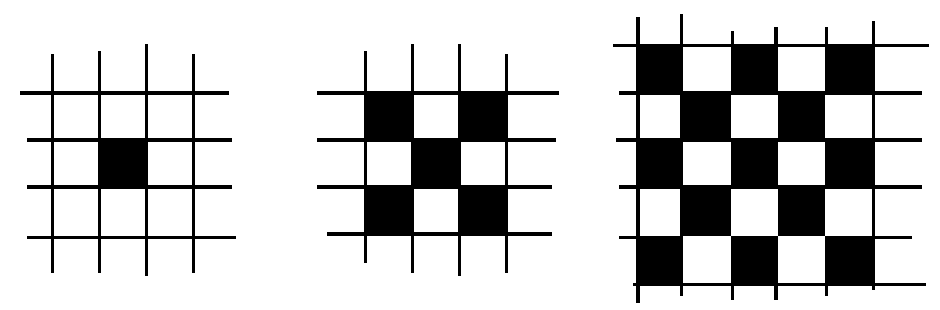

Figure 9.6: enucleating sets

problems can be rewritten as

$$
\min \left\{-\frac{2}{\varepsilon \lambda}\left(\left(L_{k-1}+\Delta L\right)^{2}-L_{k-1}^{2}\right)+\frac{1}{\tau}\left(L_{k-1}(\Delta L)^{2}+r_{k}(\Delta L)^{2}\right)\right\},
$$


with $r_{k}$ negligible as $\tau \rightarrow 0$. For the interfacial part, we have taken into account that for $\varepsilon$ small the number of squares contained in a rectangle is equal to its area divided by $2 \varepsilon^{2}$ and each of the squares gives an energy contribution of $4 \varepsilon$; for the distance part, we note that the integral can be equivalently taken on half of $Q_{k} \backslash Q_{k-1}$. Minimization in $\Delta L$ gives

$$
\frac{\Delta L}{\tau}=\frac{2}{\varepsilon \lambda}\left(1+\frac{\Delta L}{L_{k-1}}\right)
$$

Choosing $\lambda=\frac{1}{\varepsilon}$, we obtain a linear growth

$$
L(s)=2 s .
$$

What we have obtained is the description of the structure of $\varepsilon$-squares (the checkerboard one) along which the increase of the perimeter is maximal (and, in a sense, the decrease of the perimeter is maximal for the reverse-time problem).

\subsection{Reference to Chapter 9}

The literature on long-time behaviour and backward equations, even though not by the approach by minimizing movements, is huge. The long-time motion of interfaces in one space dimension by energy methods has been studied in a paper by Bronsard and Kohn [19].

Example 9.1.6 has been part of the course exam of C. Sorgentone and S. Tozza at Sapienza University in Rome.

It is a pleasure to acknowledge the suggestion of J.W. Cahn to use finite-dimensional approximations to define backward motion of sets. 


\section{Chapter 10}

\section{Stability theorems}

We now face the problem of determining conditions under which the minimizing-movement scheme commutes with $\Gamma$-convergence. Let $F_{\varepsilon} \Gamma$-converge to $F$ with initial data $x_{\varepsilon}$ converging to $x_{0}$. We have seen in Section 7.2 that by choosing suitably $\varepsilon=\varepsilon(\tau)$ the minimizing movement along the sequence $F_{\varepsilon}$ from $x_{\varepsilon}$ converges to a minimizing movement for the limit $F$ from $x_{0}$. A further issue is whether, by assuming some further properties on $F_{\varepsilon}$ we may deduce that the same thing happens for any choice of $\varepsilon$. In order to give an answer we will use results from the theory of gradient flows recently elaborated by Ambrosio, Gigli and Savaré, and by Sandier and Serfaty.

\subsection{Stability for convex energies}

We now use the theory of gradient flows to deduce stability results if the functionals satisfy some convexity assumptions. For the sake of simplicity we will assume that $X$ is a Hilbert space and all $F_{\varepsilon}$ are convex.

\subsubsection{Convergence estimates}

We first recall some results on minimizing movements for a single convex functional $F$.

Proposition 10.1.1 Let $F$ be convex, $z \in X$ and let $w$ be a minimizer of

$$
\min \left\{F_{\varepsilon}(x)+\frac{1}{2 \eta}\|x-z\|^{2}\right\}
$$

Then

$$
\|x-w\|^{2}-\|x-z\|^{2} \leq 2 \eta(F(x)-F(w))
$$

for all $x \in X$. 
Proof. We recall that the inequality

$$
\|s x+(1-s) w-z\|^{2} \leq s\|x-z\|^{2}+(1-s)\|w-z\|^{2}-s(1-s)\|x-w\|^{2}
$$

holds for all $x, w, z \in X$ and $s \in[0,1]$. Using this property and the convexity of $F$, thanks to the minimality of $w$ we have

$$
\begin{aligned}
F(w)+\frac{1}{2 \eta}\|w-z\|^{2} \leq & F(s x+(1-s) w)+\frac{1}{2 \eta}\|s x+(1-s) w-z\|^{2} \\
\leq & s F(x)+(1-s) F(w) \\
& \quad+\frac{1}{2 \eta}\left(s\|x-z\|^{2}+(1-s)\|w-z\|^{2}-s(1-s)\|x-w\|^{2}\right) .
\end{aligned}
$$

After regrouping and dividing by $s$, from this we have

$$
\frac{1}{2 \eta}\left(\|w-z\|^{2}+(1-s)\|x-w\|^{2}-\|x-z\|^{2}\right) \leq F(x)-F(w)
$$

and then the desired (10.2) after letting $s \rightarrow 0$ and dropping the positive term $\|w-z\|^{2}$.

Remark 10.1.2 Let $\left\{z_{k}\right\}=\left\{z_{k}^{\eta}\right\}$ be a minimizing scheme for $F$ from $z_{0}$ with time-step $\eta$. Then (10.2) gives

$$
\left\|x-z_{k+1}\right\|^{2}-\left\|x-z_{k}\right\|^{2} \leq 2 \eta\left(F(x)-F\left(z_{k+1}\right)\right)
$$

for all $x \in X$.

We now fix $\tau>0$ and two initial data $x_{0}$ and $y_{0}$ and want to compare the resulting $\left\{x_{k}\right\}=\left\{x_{k}^{\tau}\right\}$ obtained by iterated minimization with time-step $\tau$ and initial datum $x_{0}$ and $\left\{y_{k}\right\}=\left\{y_{k}^{\tau / 2}\right\}$ with time-step $\tau / 2$ and initial datum $y_{0}$. Note that the corresponding continuous-time interpolations are

$$
u^{\tau}(t):=x_{\lfloor t / \tau\rfloor}, \quad v^{\tau / 2}(t)=y_{\lfloor 2 t / \tau\rfloor},
$$

so that the comparison must be performed between $x_{k}$ and $y_{2 k}$.

Proposition 10.1.3 For all $j \in \mathbb{N}$ we have

$$
\left\|x_{j}-y_{2 j}\right\|^{2}-\left\|x_{0}-y_{0}\right\|^{2} \leq 2 \tau F\left(x_{0}\right) .
$$

Proof. We first give an estimate between $x_{1}$ and $y_{2}$. We first apply (10.4) with $\eta=\tau$, $z_{k}=x_{0}, z_{k+1}=y_{1}$ and $x=y_{2}$ which gives

$$
\left\|y_{2}-x_{1}\right\|^{2}-\left\|y_{2}-x_{0}\right\|^{2} \leq 2 \tau\left(F\left(y_{2}\right)-F\left(x_{1}\right)\right) .
$$


If instead we apply (10.4) with $\eta=\tau / 2, z_{k}=y_{0}, z_{k+1}=y_{1}$ and $x=x_{0}$, or $z_{k}=y_{1}$, $z_{k+1}=y_{2}$ and $x=x_{0}$ we get, respectively,

$$
\begin{aligned}
\left\|x_{0}-y_{1}\right\|^{2}-\left\|x_{0}-y_{0}\right\|^{2} & \leq \tau\left(F\left(x_{0}\right)-F\left(y_{1}\right)\right) \\
\left\|x_{0}-y_{2}\right\|^{2}-\left\|x_{0}-y_{1}\right\|^{2} & \leq \tau\left(F\left(x_{0}\right)-F\left(y_{2}\right)\right),
\end{aligned}
$$

so that, summing up,

$$
\left\|x_{0}-y_{2}\right\|^{2}-\left\|x_{0}-y_{0}\right\|^{2} \leq 2 \tau F\left(x_{0}\right)-\tau F\left(y_{1}\right)-F\left(y_{2}\right) \leq 2 \tau\left(F\left(x_{0}\right)-F\left(y_{2}\right)\right),
$$

where we have used that $F\left(y_{2}\right) \leq F\left(y_{1}\right)$ in the last inequality. Summing up (10.6) and (10.7) we obtain

$$
\left\|x_{1}-y_{2}\right\|^{2}-\left\|x_{0}-y_{0}\right\|^{2} \leq 2 \tau\left(F\left(x_{0}\right)-F\left(x_{1}\right)\right) .
$$

We now compare the later indices. We can repeat the same argument with $x_{0}$ and $y_{0}$ substituted by $x_{1}$ and $y_{2}$, so that by (10.8) we get

$$
\left\|x_{2}-y_{4}\right\|^{2}-\left\|x_{1}-y_{2}\right\|^{2} \leq 2 \tau\left(F\left(x_{1}\right)-F\left(x_{2}\right)\right),
$$

and, summing (10.8),

$$
\left\|x_{2}-y_{4}\right\|^{2}-\left\|x_{0}-y_{0}\right\|^{2} \leq 2 \tau\left(F\left(x_{0}\right)-F\left(x_{2}\right)\right) .
$$

Iterating this process we get

$$
\left\|x_{j}-y_{2 j}\right\|^{2}-\left\|x_{0}-y_{0}\right\|^{2} \leq 2 \tau\left(F\left(x_{0}\right)-F\left(x_{j}\right)\right) \leq 2 \tau F\left(x_{0}\right)
$$

as desired.

Theorem 10.1.4 Let $F$ be convex and let $F\left(x_{0}\right)<+\infty$. Then there exists a unique minimizing movement $u$ for $F$ from $x_{0}$ such that, if $u^{\tau}$ is defined by (10.5), then

$$
\left\|u^{\tau}(t)-u(t)\right\| \leq 6 \sqrt{F\left(x_{0}\right)} \sqrt{\tau}
$$

for all $t \geq 0$.

Proof. With fixed $\tau$ we first prove the convergence of $u^{2^{-j} \tau}$ as $j \rightarrow+\infty$. By Proposition 10.1.3 applied with $y_{0}=x_{0}$ and $2^{-j} \tau$ in place of $\tau$ we have

$$
\left\|u^{2^{-j} \tau}(t)-u^{2^{-j-1} \tau}(t)\right\| \leq 2^{-j / 2} \sqrt{2 \tau} \sqrt{F\left(x_{0}\right)}
$$

for all $t$. This shows the convergence to a limit $u_{\tau}(t)$, which in particular satisfies

$$
\left\|u^{\tau}(t)-u_{\tau}(t)\right\| \leq \sqrt{2} \sum_{j=0}^{\infty} 2^{-j / 2} \sqrt{\tau} \sqrt{F\left(x_{0}\right)} \leq 6 \sqrt{F\left(x_{0}\right)} \sqrt{\tau}
$$


The limit $u_{\tau}$ can be characterized as follows: with fixed $x$, inequality (10.4) applied to $z_{k}=u^{2^{-j} \tau}\left((k-1) 2^{-j} \tau\right)(k \geq 1)$ can be seen as describing in the sense of distribution the derivative

$$
\frac{d}{d t} \frac{1}{2}\left\|x-u^{2^{-j} \tau}(t)\right\|^{2} \leq \sum_{k=1}^{\infty}\left(F(x)-F\left(u^{2^{-j} \tau}\left((k-1) 2^{-j} \tau\right)\right)\right) 2^{-j} \tau \delta_{k 2^{-j} \tau} .
$$

Note in fact that $x \mapsto \frac{1}{2}\left\|x-u^{2^{-j} \tau}\right\|^{2}$ is a piecewise-constant function with discontinuities in $2^{-j} \tau \mathbb{Z}$, whose size is controlled by (10.4). Since the measures

$$
\mu_{j}=\sum_{k=1}^{\infty} 2^{-j} \tau \delta_{k 2^{-j} \tau}
$$

converge to the Lebesgue measure, and $u^{2^{-j} \tau}(t) \rightarrow u_{\tau}(t)$ for all $t$, so that by the lower semicontinuity of $F$

$$
F\left(u_{\tau}(t)\right) \leq \liminf _{j \rightarrow+\infty} F\left(u^{2^{-j}} \tau(t)\right)
$$

we deduce that

$$
\frac{d}{d t} \frac{1}{2}\left\|x-u_{\tau}(t)\right\|^{2} \leq F(x)-F\left(u_{\tau}(t)\right)
$$

for all $x$. Equation (10.15) is sufficient to characterize $u_{\tau}$. We only sketch the argument: suppose otherwise that (10.15) is satisfied by some other $v(t)$. Then we have

$$
\left\langle x-u_{\tau}, \nabla u_{\tau}\right\rangle \leq F(x)-F\left(u_{\tau}\right) \quad \text { and } \quad\langle x-v, \nabla v\rangle \leq F(x)-F(v)
$$

for all $x$. Inserting $x=v(t)$ and $x=u_{\tau}(t)$ respectively, and summing the two inequalities we have

$$
\frac{d}{d t} \frac{1}{2}\left\|v(t)-u_{\tau}(t)\right\|^{2}=\left\langle v-u_{\tau}, \nabla v-\nabla u_{\tau}\right\rangle \leq 0 .
$$

Since $v(0)=u_{\tau}(0)$ we then have $v=u_{\tau}$.

This argument shows that $u=u_{\tau}$ does not depend on $\tau$. We then have the convergence of the whole sequence, and (10.13) gives the desired estimate of $\left\|u^{\tau}-u\right\|$.

\subsubsection{Stability along sequences of convex energies}

From the estimates in the previous section, and the convergence argument in Section 7.2 we can deduce the following stability results.

Theorem 10.1.5 Let $F_{\varepsilon}$ be a sequence of lower-semicontinuous coercive positive convex energies $\Gamma$-converging to $F$, and let $x_{0}^{\varepsilon} \rightarrow x_{0}$ with $\sup _{\varepsilon} F_{\varepsilon}\left(x_{0}^{\varepsilon}\right)<+\infty$. Then

(i) for every choice of $\tau$ and $\varepsilon$ converging to 0 the family $u^{\varepsilon}$ introduced in Definition 7.1.1 converges to the unique u given by Theorem 10.1.4;

(ii) the sequence of minimizing movements $u_{\varepsilon}$ for $F_{\varepsilon}$ from $x_{0}^{\varepsilon}$ (given by Theorem 10.1.4 with $F_{\varepsilon}$ in place of $F$ ) also converge to the same minimizing movement $u$. 
Proof. We first show (ii). Indeed, by the estimate in Theorem 10.1.4 we have that, after defining $u_{\varepsilon}^{\tau}$ following the notation of that theorem,

$$
\left\|u^{\tau}-u\right\|_{\infty} \leq M \sqrt{\tau}, \quad\left\|u_{\varepsilon}^{\tau}-u_{\varepsilon}\right\|_{\infty} \leq M \sqrt{\tau}
$$

where

$$
M=6 \sup _{\varepsilon} F_{\varepsilon}\left(x_{0}^{\varepsilon}\right) .
$$

In order to show that $u_{\varepsilon} \rightarrow u$ it suffices to show that $u_{\varepsilon}^{\tau} \rightarrow u^{\tau}$ for fixed $\tau$. That has already been noticed to hold in Section 7.2.

In order to prove (i) it suffices to use the triangular inequality

$$
\left\|u_{\varepsilon}^{\tau}-u\right\| \leq\left\|u_{\varepsilon}^{\tau}-u_{\varepsilon}\right\|+\left\|u_{\varepsilon}-u\right\| \leq M \sqrt{\tau}+o(1)
$$

by Theorem 10.1 .4 and (ii).

Remark 10.1.6 (compatible topologies) We may weaken the requirement that $F_{\varepsilon}$ be equi-coercive with respect to the $X$-convergence. It suffices to require that the $\Gamma$-limit be performed with respect to a topology compatible with the $X$-norm; i.e., such that the $\Gamma$-convergence $F_{\varepsilon} \rightarrow F$ ensures that $F_{\varepsilon}(x)+C\left\|x-x_{0}\right\|^{2} \Gamma$-converges to $F(x)+C\left\|x-x_{0}\right\|^{2}$ for fixed $C$ and $x_{0}$, and with respect to which these energies are equi-coercive. In this way we still have $u_{\varepsilon}^{\tau} \rightarrow u^{\tau}$ in the proof above.

Example 10.1.7 (parabolic homogenization) We can consider $X=L^{2}(0, T)$,

$$
F_{\varepsilon}(u)=\int_{0}^{T} a\left(\frac{x}{\varepsilon}\right)\left|u^{\prime}\right|^{2} d x, \quad F(u)=\underline{a} \int_{0}^{T}\left|u^{\prime}\right|^{2} d x
$$

with the notation of Section 1.4. We take as initial datum $u_{0}$ independent of $\varepsilon$. Since all functionals are convex, lower semicontinuous and coercive, from Theorem 10.1.5 we deduce the converge of the corresponding minimizing movements. From this we deduce the convergence of the solutions of the parabolic problem with oscillating coefficients

$$
\left\{\begin{array}{l}
\frac{\partial u_{\varepsilon}}{\partial t}=\frac{\partial}{\partial x}\left(a\left(\frac{x}{\varepsilon}\right) \frac{\partial u_{\varepsilon}}{\partial x}\right) \\
u_{\varepsilon}(x, 0)=u_{0}(x)
\end{array}\right.
$$

to the solution of the heat equation

$$
\left\{\begin{array}{l}
\frac{\partial u}{\partial t}=\underline{a} \frac{\partial^{2} u}{\partial x^{2}} \\
u_{\varepsilon}(x, 0)=u_{0}(x) .
\end{array}\right.
$$


Example 10.1.8 (high-contrast media) We consider a discrete system parameterized on $\{0, \ldots, N\}$ with $N$ even. We set $\varepsilon=1 / N$ and consider the energies

$$
F_{\varepsilon}(u)=\frac{1}{2} \sum_{l=1}^{N / 2} \varepsilon\left|\frac{u_{2 l}-u_{2 l-2}}{\varepsilon}\right|^{2}+\frac{c_{\varepsilon}}{2} \sum_{j=1}^{N} \varepsilon\left|\frac{u_{j}-u_{j-1}}{\varepsilon}\right|^{2}
$$

with a periodic boundary condition $u_{N}=u_{0}$.

This is a simple model where two elliptic energies interact possibly on different scales. The critical scale is when

$$
c_{\varepsilon}=\varepsilon^{2},
$$

condition that will be assumed in the rest of the example. The first sum is a strong next-to-nearest-neighbor interaction between even points, and the second one is a weak nearest-neighbor interaction between all points.

Upon identifying $u_{i}$ with the piecewise-constant function $u \in L^{2}(0,1)$ with $u(x)=u_{\lfloor x / \varepsilon\rfloor}$ we may regard $F_{\varepsilon}$ as defined on $X=L^{2}(0,1)$ and consider the minimizing movement of $F_{\varepsilon}$ with respect to the $L^{2}$-norm, which we can write

$$
\|u\|^{2}=\sum_{j=1}^{N} \varepsilon\left|u_{i}\right|^{2}
$$

on the domain of $F_{\varepsilon}$, so that the iterated minimum problem giving $u^{k}$ reads

$$
\min \left\{\frac{1}{2} \sum_{l=1}^{N / 2} \varepsilon\left|\frac{u_{2 l}-u_{2 l-2}}{\varepsilon}\right|^{2}+\frac{1}{2} \sum_{j=1}^{N} \varepsilon^{3}\left|\frac{u_{j}-u_{j-1}}{\varepsilon}\right|^{2}+\frac{1}{2 \tau} \sum_{j=1}^{N} \varepsilon\left(u_{j}-u_{j}^{k-1}\right)^{2}\right\} .
$$

We consider as initial datum (the sampling on $\varepsilon \mathbb{Z} \cap[0,1]$ of) a smooth 1-periodic datum $u^{0}$ (for simplicity independent of $\varepsilon$ ).

Since all $F_{\varepsilon}$ are convex, we may describe their minimizing movement through the gradient flow of their $\Gamma$-limit. Since $F_{\varepsilon}$ is not equi-coercive with respect to the $L^{2}$ norm, we have to choose a different topology for the $\Gamma$-limit.

Among the different choices we may consider the following two.

(1) We choose the strong $L^{2}$-convergence of the even piecewise-constant interpolations only; i.e.,

$$
\|u-v\|_{\text {even }}^{2}=\sum_{j=1}^{N} \varepsilon\left|u_{2 j}-v_{2 j}\right|^{2} .
$$

Note that $F_{\varepsilon}$ are equi-coercive and their $\Gamma$-limit is simply

$$
F^{s}(u)=\int_{0}^{1}\left|u^{\prime}\right|^{2} d x
$$


To check this it suffices to remark that, if we consider the even piecewise-affine interpolation $\widetilde{u}$ of $u_{i}$, then we have

$$
\sum_{l=1}^{N / 2} \varepsilon\left|\frac{u_{2 l}-u_{2 l-2}}{\varepsilon}\right|^{2}=2 \sum_{l=1}^{N / 2} 2 \varepsilon\left|\frac{u_{2 l}-u_{2 l-2}}{2 \varepsilon}\right|^{2}=2 \int_{0}^{1}\left|\widetilde{u}^{\prime}\right|^{2} d x,
$$

so that $F^{s}$ is a lower bound, while a recovery sequence is simply obtained by taking $u_{\varepsilon}$ the interpolation of $u$, for which

$$
F_{\varepsilon}\left(u_{\varepsilon}\right)=\int_{0}^{1}\left|u^{\prime}\right|^{2} d x+\frac{\varepsilon^{2}}{2} \int_{0}^{1}\left|u^{\prime}\right|^{2} d x+o(1) .
$$

(2) We choose the strong $L^{2}$-convergence of the even piecewise-constant interpolations and the weak $L^{2}$-convergence of the odd piecewise-constant interpolations. A function $u$ is then identified with a pair $\left(u_{e}, u_{o}\right)$ (even and odd piecewise-constant interpolations), so that

$$
F_{\varepsilon}(u)=F^{w}\left(u_{e}, u_{o}\right):=\int_{0}^{1}\left|u_{e}^{\prime}\right|^{2} d x+\frac{1}{2} \int_{0}^{1}\left|u_{e}-u_{o}\right|^{2} d x .
$$

The functional $F^{w}$ thus defined is the $\Gamma$-limit in this topology, which is compatible with the $L^{2}$-distance (interpreted as the sum of the $L^{2}$-distances of the even/odd piecewise-constant interpolations).

We can apply Theorem 10.1.5, together with Remark 10.1.6, and deduce that the minimizing movement for $F_{\varepsilon}$ is given by the solution $\left(u_{e}, u_{o}\right)=\left(u_{e}(x, t), u_{o}(x, t)\right)$ of the gradient flow for $F^{w}$, which is

$$
\left\{\begin{array}{l}
\frac{\partial u_{e}}{\partial t}=2 \frac{\partial^{2} u_{e}}{\partial x^{2}}-u_{e}+u_{o} \\
\frac{\partial u_{o}}{\partial t}=u_{o}-u_{e} \\
u_{o}(x, 0)=u_{e}(x, 0)=u^{0}(x)
\end{array}\right.
$$

with periodic boundary conditions for $u_{e}$.

Note that $F^{s}$ is not compatible with the $L^{2}$-norm since it does not contain the odd interpolations, and its gradient flow is simply a heat equation. Note however that we may use $u_{e}$ as a single parameter with respect to which to describe the minimizing movement of $F_{\varepsilon}$, as suggested by the choice of $F^{s}$ as $\Gamma$-limit. Indeed, we may integrate the second equation of the system above expressing $u_{o}$ in terms of $u_{e}$. Plugging its expression in the first equation we obtain the integro-differential problem satisfied by $u_{e}$

$$
\left\{\begin{array}{l}
\frac{\partial u(x, t)}{\partial t}=2 \frac{\partial^{2} u(x, t)}{\partial x^{2}}-u(x, t)+u^{0}(x) e^{-t}+\int_{0}^{t} e^{s-t} u(x, s) d s \\
u(x, 0)=u^{0}(x)
\end{array}\right.
$$

with periodic boundary conditions. 


\subsection{Sandier-Serfaty theory}

We have already remarked that for some non-convex problems minimizing movements commute with $\Gamma$-convergence, as for approximations of the Mumford-Shah functional. We conclude this section by giving a brief (and simplified) account of another very fruitful approach to gradient flows that allows to prove the stability of certain solutions with respect to $\Gamma$-convergence related to non-convex energies.

\subsubsection{Convergence of gradient flows}

We consider a family of Hilbert spaces $X_{\varepsilon}$ and functionals $F_{\varepsilon}: X_{\varepsilon} \rightarrow(-\infty,+\infty]$, which are $C^{1}$ on their domain. We denote by $\nabla_{X_{\varepsilon}} F_{\varepsilon}$ the gradient of $F_{\varepsilon}$ in $X_{\varepsilon}$.

Definition 10.2.1 Let $T>0$; we say that $u_{\varepsilon} \in H^{1}\left([0, T) ; X_{\varepsilon}\right)$ is a a.e. solution for the gradient flow of $F_{\varepsilon}$ if

$$
\frac{\partial u_{\varepsilon}}{\partial t}=-\nabla_{X_{\varepsilon}} F_{\varepsilon}\left(u_{\varepsilon}\right)
$$

almost everywhere on $(0, T)$. Such solution for the a gradient flow is conservative if

$$
F_{\varepsilon}\left(u_{\varepsilon}(0)\right)-F_{\varepsilon}\left(u_{\varepsilon}(s)\right)=\int_{0}^{s}\left\|\frac{\partial u_{\varepsilon}}{\partial t}\right\|_{X_{\varepsilon}}^{2} d t
$$

for all $\tau \in(0, T)$.

We suppose that there exists a Hilbert space $X$ and a notion of metrizable convergence $x_{\varepsilon} \rightarrow x$ of families of elements of $X_{\varepsilon}$ to an element of $X$. With respect to that convergence, we suppose that $F_{\varepsilon} \Gamma$-converge to a functional $F$, which is also $C^{1}$ on its domain.

Theorem 10.2.2 (Sandier-Serfaty Theorem) Let $F_{\varepsilon}$ and $F$ be as above with $F_{\varepsilon} \Gamma$ converging to $F$, let $u_{\varepsilon}$ be a family of conservative solutions for the gradient flow of $F_{\varepsilon}$ with initial data $u_{\varepsilon}(0)=u^{\varepsilon}$ converging to $u^{0}$. Suppose furthermore that

- (well-preparedness of initial data) $u^{\varepsilon}$ is a recovery sequence for $F\left(u^{0}\right)$;

- (lower bound) upon subsequences $u_{\varepsilon}$ converges to some $u \in H^{1}((0, T) ; X)$ and

$$
\begin{gathered}
\liminf _{\varepsilon \rightarrow 0} \int_{0}^{s}\left\|\frac{\partial u_{\varepsilon}}{\partial t}\right\|_{X_{\varepsilon}}^{2} d t \geq \int_{0}^{s}\left\|\frac{\partial u}{\partial t}\right\|_{X}^{2} d t \\
\liminf _{\varepsilon \rightarrow 0}\left\|\nabla_{X_{\varepsilon}} F_{\varepsilon}\left(u_{\varepsilon}(s)\right)\right\|_{X_{\varepsilon}}^{2} \geq\left\|\nabla_{X} F(u(s))\right\|_{X}^{2}
\end{gathered}
$$

for all $s \in(0, T)$.

Then $u$ is a solution for the gradient flow of $F$ with initial datum $u^{0}, u_{\varepsilon}(t)$ is a recovery sequence for $F(u(t)$ for all $t$ and the inequalities in (10.16) and (10.17) are equalities. 
Proof. Using the fact that $u_{\varepsilon}$ is conservative and that for all $t$

$$
\left\|\nabla_{X_{\varepsilon}} F_{\varepsilon}\left(u_{\varepsilon}\right)+\frac{\partial u_{\varepsilon}}{\partial t}\right\|_{X_{\varepsilon}}^{2}=0
$$

and hence

$$
-\left\langle\nabla_{X_{\varepsilon}} F_{\varepsilon}\left(u_{\varepsilon}(t)\right), \frac{\partial u_{\varepsilon}}{\partial t}\right\rangle=\frac{1}{2}\left(\left\|\nabla_{X_{\varepsilon}} F_{\varepsilon}\left(u_{\varepsilon}(t)\right)\right\|_{X_{\varepsilon}}^{2}+\left\|\frac{\partial u_{\varepsilon}}{\partial t}\right\|_{X_{\varepsilon}}^{2}\right) .
$$

We then get

$$
\begin{aligned}
F_{\varepsilon}\left(u_{\varepsilon}(0)\right)-F_{\varepsilon}\left(u_{\varepsilon}(t)\right) & =\int_{0}^{t}\left\|\frac{\partial u_{\varepsilon}}{\partial t}\right\|_{X_{\varepsilon}}^{2} d s \\
& =-\int_{0}^{t}\left\langle\nabla_{X_{\varepsilon}} F_{\varepsilon}\left(u_{\varepsilon}\right), \frac{\partial u_{\varepsilon}}{\partial t}\right\rangle_{X_{\varepsilon}} d s \\
& =\frac{1}{2} \int_{0}^{t}\left(\left\|\nabla_{X_{\varepsilon}} F_{\varepsilon}\left(u_{\varepsilon}\right)\right\|_{X_{\varepsilon}}^{2}+\left\|\frac{\partial u_{\varepsilon}}{\partial t}\right\|_{X_{\varepsilon}}^{2}\right) d s
\end{aligned}
$$

By the lower-bound assumption then we have

$$
\begin{aligned}
\liminf _{\varepsilon \rightarrow 0}\left(F_{\varepsilon}\left(u_{\varepsilon}(0)\right)-F_{\varepsilon}\left(u_{\varepsilon}(t)\right)\right) & \geq \frac{1}{2} \int_{0}^{t}\left(\left\|\nabla_{X} F(u)\right\|_{X}^{2}+\left\|\frac{\partial u}{\partial t}\right\|_{X}^{2}\right) d s \\
& \geq-\int_{0}^{t}\left\langle\nabla_{X} F(u), \frac{\partial u}{\partial t}\right\rangle_{X} d s .
\end{aligned}
$$

The last term equals

$$
-\int_{0}^{t} \frac{d}{d t} F(u) d s=F(u(0))-F(u(t))
$$

so that we have

$$
\liminf _{\varepsilon \rightarrow 0}\left(F_{\varepsilon}\left(u_{\varepsilon}(0)\right)-F_{\varepsilon}\left(u_{\varepsilon}(t)\right)\right) \geq F(u(0))-F(u(t)) .
$$

Since $u_{\varepsilon}(0)$ is a recovery sequence for $F(u(0))$ we then have

$$
F(u(t)) \geq \limsup _{\varepsilon \rightarrow 0} F_{\varepsilon}\left(u_{\varepsilon}(t)\right),
$$

so that $u_{\varepsilon}(t)$ is a recovery sequence for $u(t)$ and indeed we have equality in (10.19) and hence both inequalities in (10.18) are equalities. The second one of those shows that

$$
\left\|\nabla_{X} F(u)+\frac{\partial u}{\partial t}\right\|_{X}^{2}=0
$$

for all $t$, and hence the thesis. 
Example 10.2.3 (Ginzburg-Landau vortices) The theory outlined above has been successfully applied by Sandier and Serfaty to obtain the motion of vortices as the limit of the gradient flows of Ginzburg-Landau energies. We give a short account of their setting without entering into details.

Let $\Omega$ be a bounded regular open subset of $\mathbb{R}^{2}$ and $N \in \mathbb{N}$; the Hilbert spaces $X_{\varepsilon}$ and $X$ are chosen as

$$
X_{\varepsilon}=L^{2}\left(\Omega ; \mathbb{R}^{2}\right), \quad X=\mathbb{R}^{2 N}
$$

with scalar products

$$
\langle u, v\rangle_{X_{\varepsilon}}=\frac{1}{|\log \varepsilon|} \int_{\Omega}\langle u(x), v(x)\rangle_{\mathbb{R}^{2}} d x, \quad\langle x, y\rangle_{X}=\frac{1}{\pi}\langle x, y\rangle_{\mathbb{R}^{2 N}},
$$

respectively.

The energies $F_{\varepsilon}: H^{1}\left(\Omega ; \mathbb{R}^{2}\right) \rightarrow \mathbb{R}$ are defined as

$$
F_{\varepsilon}(u)=\frac{1}{2} \int_{\Omega}\left(|\nabla u|^{2}+\frac{1}{\varepsilon^{2}}\left(1-|u|^{2}\right)^{2}\right) d x .
$$

The convergence of $u_{\varepsilon}$ is defined as follows: if we write in polar coordinates

$$
u_{\varepsilon}(x)=\rho_{\varepsilon}(x) e^{i \varphi_{\varepsilon}(x)}
$$

then $u_{\varepsilon} \rightarrow\left(x^{1}, \ldots, x^{N}\right)$ if we have

$$
\lim _{\varepsilon \rightarrow 0} \operatorname{curl}\left(\rho_{\varepsilon}^{2} \nabla \varphi_{\varepsilon}\right)=2 \pi \sum_{j=1}^{N} d_{j} \delta_{x^{j}}
$$

weak* in the sense of measures for some integers $d_{j}$, where $\operatorname{curl}\left(A_{1}, A_{2}\right)=\frac{\partial A_{1}}{\partial x_{2}}-\frac{\partial A_{2}}{\partial x_{1}}$. This convergence describes the location of vortices at the points $x^{j}$ with a degree $d_{j}$. For $u_{\varepsilon}(x) \rightarrow x /|x|$ we have $N=1, x^{1}=0$ and $d_{1}=1$.

It can be proved that there exists a function $W=W_{\left(d_{1}, \ldots, d_{N}\right)}$ such that

$$
\Gamma-\lim _{\varepsilon \rightarrow 0}\left(F_{\varepsilon}(u)-\pi N|\log \varepsilon|\right)=W\left(x^{1}, \ldots, x^{N}\right) .
$$

The function $W$ can be characterized in terms of the Green function of $\Omega$. Its precise definition is not relevant to this example.

The well-preparedness condition for the initial data amounts to requiring that

$$
u_{\varepsilon}^{0} \rightarrow\left(x_{0}^{1}, \ldots, x_{0}^{N}\right) \quad \text { and } d_{j} \in\{-1,1\} .
$$


Under these conditions we may apply Theorem 10.2.2 to the scaled energies $F_{\varepsilon}-$ $\pi N|\log \varepsilon|$. This yields solutions $u_{\varepsilon}=u_{\varepsilon}(x, t)$ to the equation

$$
\left\{\begin{array}{l}
\frac{1}{|\log \varepsilon|} \frac{\partial u_{\varepsilon}}{\partial t}=\Delta u+\frac{1}{\varepsilon^{2}} u_{\varepsilon}\left(1-\left|u_{\varepsilon}\right|^{2}\right) \quad \text { in } \Omega \\
\frac{\partial u_{\varepsilon}}{\partial n}=0 \quad \text { on } \partial \Omega \\
u_{\varepsilon}(x, 0)=u_{\varepsilon}^{0}(x)
\end{array}\right.
$$

converging to $x(t)=\left(x^{1}(t), \ldots, x^{N}(t)\right)=\left(x_{1}(t), \ldots, x_{2 N}(t)\right)$. The limit vortices move following the system of ODE

$$
\frac{d x_{i}}{d t}=-\frac{1}{\pi} \frac{\partial W(x)}{\partial x_{i}}
$$

This description is valid until the first collision time $T^{*}$ when $x_{j}\left(T^{*}\right)=x_{k}\left(T^{*}\right)$ for some $j$ and $k$ with $j \neq k$.

\subsubsection{Convergence of stable critical points}

The Sandier-Serfaty approach can be extended to analyze the convergence of critical points, for which Theorem 10.2.2 is trivial.

Theorem 10.2.4 Let $u_{\varepsilon}$ be a family of critical points of $F_{\varepsilon}$ with $u_{\varepsilon} \rightarrow u$, such that the following holds: for any $V \in X$, we can find $v_{\varepsilon}(t)$ defined in a neighborhood of $t=0$, such that $\partial_{t} v_{\varepsilon}(0)$ depends on $V$ in a linear and one-to-one manner, and

$$
\begin{aligned}
& v_{\varepsilon}(0)=u_{\varepsilon}(0) \\
& \lim _{\varepsilon \rightarrow 0} \frac{d}{d t} F_{\varepsilon}\left(v_{\varepsilon}(t)\right)_{\mid t=0}=\frac{d}{d t} F(u+t V)_{\mid t=0}=\langle d F(u), V\rangle \\
& \lim _{\varepsilon \rightarrow 0} \frac{d^{2}}{d t^{2}} F_{\varepsilon}\left(v_{\varepsilon}(t)\right)_{\mid t=0}=\frac{d^{2}}{d t^{2}} F(u+t V)_{\mid t=0}=Q(u)(V) .
\end{aligned}
$$

Then

- if (10.20)-(10.21) are satisfied, then $u$ is a critical point of $F$

- if (10.20)-(10.21)-(10.22) are satisfied, then if $u_{\varepsilon}$ are critical points of $E_{\varepsilon}$ with positive Hessian, $u$ is a critical point of $F$ with positive Hessian.

We do not enter in the details of this result, but only remark that in this case the $\Gamma$ convergence of $F_{\varepsilon}$ to $F$ is not required, in analogy to the conditions analyzed in Section 5 . 


\subsection{References to Chapter 10}

The results in Section 10.1.1 and part (ii) of Theorem 10.1.4 are a simplified version of the analogous results for geodesic-convex energies in metric spaces that can be found in the notes by Ambrosio and Gigli [6].

The result by Sandier and Serfaty (with weaker hypotheses than those reported here) is contained in the seminal paper [41]. An account of their approach is contained in the notes by Serfaty [43]. The convergence of stable points has been considered by Serfaty in [42] and further analyzed by Jerrard and Sternberg in [31]. 


\section{Bibliography}

[1] F. Almgren and J.E. Taylor. Flat flow is motion by crystalline curvature for curves with crystalline energies. J. Differential Geom. 42 (1995), 1-22

[2] F. Almgren, J.E. Taylor, and L. Wang. Curvature driven flows: a variational approach. SIAM J. Control Optim. 50 (1983), 387-438.

[3] L. Ambrosio. Minimizing movements. Rend. Accad. Naz. Sci. XL Mem. Mat. Appl. 19 (1995), 191-246.

[4] L. Ambrosio and A. Braides. Energies in SBV and variational models in fracture mechanics. In Homogenization and Applications to Material Sciences, (D. Cioranescu, A. Damlamian, P. Donato eds.), GAKUTO, Gakkōtosho, Tokio, Japan, 1997, p. 1-22,

[5] L. Ambrosio, N. Fusco and D. Pallara. Functions of Bounded Variation and Free Discontinuity Problems, Oxford University Press, Oxford, 2000.

[6] L. Ambrosio and N. Gigli. A user's guide to optimal transport, in Modelling and $O p$ timisation of Flows on Networks (B. Piccoli and M. Rascle eds.) Lecture Notes in Mathematics. Springer, Berlin, 2013, pp. 1-155.

[7] L. Ambrosio, N. Gigli and G. Savaré, Gradient flows in metric spaces and in the space of probability measures, Lectures in Mathematics ETH Zürich, Birkhhäuser, Basel, 2008.

[8] B. Bourdin, G.A. Francfort, and J.-J. Marigo. The Variational Approach to Fracture. Journal of Elasticity 91 (2008), 5-148.

[9] A. Braides. Г-convergence for Beginners. Oxford University Press, 2002.

[10] A. Braides. Approximation of Free-Discontinuity Problems. Lecture Notes in Mathematics 1694, Springer Verlag, Berlin, 1998.

[11] A. Braides. A Handbook of $\Gamma$-convergence. In Handbook of Partial Differential Equations. Stationary Partial Differential Equations, Volume 3 (M. Chipot and P. Quittner, eds.). Elsevier, 2006, p. 101-213.

[12] A. Braides, G. Dal Maso and A. Garroni. Variational formulation of softening phenomena in fracture mechanics: the one-dimensional case. Arch. Rational Mech. Anal. 146 (1999), 23-58. 
[13] A. Braides A. and A. Defranceschi. Homogenization of Multiple Integrals. Oxford University Press, Oxford, 1998.

[14] A. Braides, M.S. Gelli, and M. Novaga. Motion and pinning of discrete interfaces. Arch. Ration. Mech. Anal. 95 (2010), 469-498.

[15] A. Braides and C.J. Larsen. $\Gamma$-convergence for stable states and local minimizers. Ann. Scuola Norm. Sup. Pisa 10 (2011), 193-206

[16] A. Braides, A.J. Lew and M. Ortiz. Effective cohesive behavior of layers of interatomic planes. Arch. Ration. Mech. Anal.180 (2006), 151-182.

[17] A. Braides and G. Scilla. Motion of discrete interfaces in periodic media. Preprint, 2013.

[18] A. Braides and L. Truskinovsky. Asymptotic expansions by Gamma-convergence. Cont. Mech. Therm. 20 (2008), 21-62

[19] L. Bronsard and R.V. Kohn. On the slowness of phase boundary motion in one space dimension. Comm. Pure Appl. Math. 43 (1990), 983-997.

[20] A. Chambolle and F. Doveri. Minimizing movements of the Mumford and Shah energy. Discr. Cont. Dynamical Syst. 3 (1997), 153-174.

[21] G. Dal Maso. An Introduction to Г-convergence. Birkhäuser, Boston, 1993.

[22] E. De Giorgi. New problems on minimizing movements. In E. De Giorgi. Selected Papers. Springer, Berlin, 2006.

[23] M. Focardi. $\Gamma$-convergence: a tool to investigate physical phenomena across scales, Math. Mod. Meth. Appl. Sci. 35 (2012), 1613-1658

[24] G. Francfort and A. Garroni, A variational view of brittle damage evolution, Arch. Rational Mech. Anal. 182 (2006), 125-152.

[25] G. A. Francfort and C, J. Larsen. Existence and convergence for quasi-static evolution in brittle fracture. Comm. Pure Appl. Math. 56 (2003), 1465-1500.

[26] G. Francfort and J.-J. Marigo. Stable damage evolution in a brittle continuous medium, Eur. J. Mech. A/Solids, 12 (1993), 149-189.

[27] G. Francfort and J.-J. Marigo. Revisiting brittle fracture as an energy minimization problem. Journal of Mechanics and Physics of Solids 46 (1998), 1319-1342.

[28] A. Garroni and C. J. Larsen: Threshold-based quasi-static brittle damage evolution, Arch. Rational Mech. Anal., Vol. 194 (2009), no. 2, 585-609

[29] M. Gobbino. Gradient flow for the one-dimensional Mumford-Shah functional. Ann. Scuola Norm. Sup. Pisa (IV) 27 (1998), 145-193. 
[30] R.V. Kohn and P. Sternberg, Local minimizers and singular perturbations. Proc. Roy. Soc. Edinburgh A, 111 (1989), 69-84.

[31] R.L. Jerrard and P. Sternberg, Critical points via G convergence: general theory and applications. J. Europ. Math. Society 11 (2009), 705-753.

[32] C. J. Larsen, Epsilon-stable quasi-static brittle fracture evolution, Comm. Pure Appl. Math. 63 (2010), 630-654,

[33] F. Maggi. Sets of Finite Perimeter and Geometric Variational Problems: an introduction to Geometric Measure Theory. Cambridge University Press, Cambridge, 2012.

[34] A. Mielke. Evolution of Rate-Independent Systems, in Handbook of Partial Differential Equations. Evolutionary Equations, Volume 2 (C.M. Dafermos and E. Feireisl eds), Elsevier, 2005, pp. 461-560.

[35] A. Mielke. Emergence of rate-independent dissipation from viscous systems with wiggly energies. Continuum Mech. Thermodyn. 24 (2012), 591-606

[36] A. Mielke, T. Roubiček, and U. Stefanelli. Г-limits and relaxations for rate-independent evolutionary problems. Calc. Var. Part. Diff. Equ. 31 (2008), 387-416, 2008.

[37] A. Mielke and F. Theil. On rate-independent hysteresis models. Nonlinear Diff. Eq. Appl. (NoDEA) 11 (2004) 151-189.

[38] A. Mielke, F. Theil, and V.I. Levitas. A variational formulation of rate-independent phase transformations using an extremum principle. Arch. Rational Mech. Anal. 162 (2002), 137-177.

[39] A. Mielke and L.Truskinovsky. From discrete visco-elasticity to continuum rate-independent plasticity: rigorous results. Arch. Rational Mech. Anal. 203 (2012), 577-619

[40] G. Puglisi and L. Truskinovsky. Thermodynamics of rate-independent plasticity. Journal of Mechanics and Physics of Solids 53 (2005), 655-679.

[41] E. Sandier and S. Serfaty, Gamma-Convergence of Gradient Flows and Application to Ginzburg-Landau, Comm. Pure Appl. Math. 57 (2004), 1627-1672.

[42] S. Serfaty, Stability in 2D Ginzburg-Landau passes to the limit, Indiana Univ. Math. J. 54 (2005), 199-222,

[43] S. Serfaty, Gamma-convergence of gradient flows and applications to Ginzburg-Landau vortex dynamics. in Topics on concentration phenomena and problems with multiple scales (A. Braides and V. Chiadò Piat eds.). Lect. Notes Unione Mat. Ital. 2, Springer, Berlin, 2006, pp. 267-292. 


\section{Index}

Almgren, Taylor and Wang theory, 124

compactness property, 20

continuous convergence, 18

convex envelope, 20

critical points, 157

crystalline perimeter, 125

dissipation, 31

energy conservation, 47

energy inequality, 46

equip-coercive, 16

equivalence by $\Gamma$-convergence, 55

flat flow, 125

Fundamental Theorem of $\Gamma$-convergence, 20

Gamma-limit, 17

Ginzburg-Landau vortices, 156

global stability, 46

gradient flow, 154

Gradient theory of phase transition, 27

Griffith fracture energy, 30

homogenization, 21

hysteresis, 47

irreversibility, 34

isolated local minimizer, 67

Lennard Jones potential, 28

liminf inequality, 15

limsup inequality, 16

local minimizer, 55 lower bound, 15

lower Gamma-limit, 19

lower-semicontinuous envelope, 19

minimizing movement, 89

minimizing movement along a sequence, 100

motion by crystalline curvature, 125

motion by mean curvature, 121

Mumford-Shah functional, 30, 139

mutual recovery sequence, 49

perimeter, 65

Perona-Malik functional, 142

pinning, 113

pinning threshold, 111

random minimizing movements, 117

rate-independence, 47

recovery sequence, 17

relaxed evolution, 105

relaxed total energy, 44

Riemann-Lebesgue lemma, 22

sets of finite perimeter, 28

slide, 79

stable evolution, 86

stable functionals, 81

stable point, 79

threshold formulation, 35

uniformly stable sequence, 79

upper bound, 16

upper Gamma-limit, 19 